\title{
Cellular and transcriptional profiling of hypoxic cells in the brain
}

\author{
Dissertation \\ for the award of the degree \\ "Doctor of Philosophy" (Ph.D.) \\ of the Georg-August-Universität Göttingen
}

within the Molecular Medicine doctoral program of the Göttingen Graduate School for Neurosciences, Biophysics, and Molecular Biosciences (GGNB) and of the Georg-August University School of Science (GAUSS)

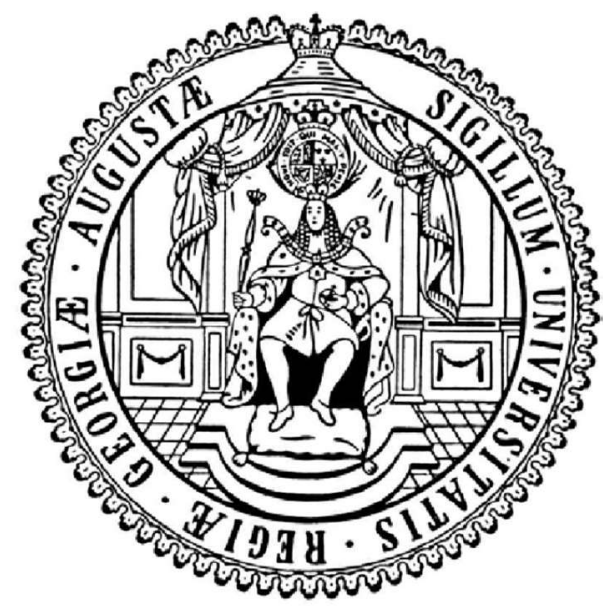

submitted by

Umer Javed Butt

born in Gujar Khan, Pakistan

Göttingen, 2019 



\section{Doctoral Thesis Committee:}

Prof. Dr. Dr. Hannelore Ehrenreich (1st referee)

Clinical Neuroscience

Max Planck Institute of Experimental Medicine, Göttingen

Prof. Dr. Dörthe M. Katschinski (2nd referee)

Department of Cardiovascular Physiology

University Medical center, Göttingen

\section{Prof. Dr. Tiago Fleming Outeiro}

Department of Experimental Neurodegeneration

University Medical center, Göttingen

\section{Prof. Klaus-Armin Nave, PhD}

Department of Neurogenetics

Max Planck Institute of Experimental Medicine, Göttingen

\section{Prof. Dr. Susann Boretius}

Functional Imaging, German Primate Center

Georg-August-University of Göttingen

PD Dr. Hauke Werner

Department of Neurogenetics

Max Planck Institute of Experimental Medicine, Göttingen

Date of submission of thesis: 22.10.2019

Date of the oral examination: 09.12.2019 



\section{Declaration}

I hereby declare that the thesis "Cellular and transcriptional profiling of hypoxic cells in the brain" has been written independently and with no other sources and aids than quoted.

Umer Javed Butt

Göttingen, 22.10.2019 



\section{Acknowledgement}

All praises to "ALLAH", the Lord of the worlds, and His Holy Prophet, Hazrat

$$
\text { Muhammad (S.A.W). }
$$

First and foremost I am grateful to my supervisor, Prof. Hannelore Ehrenreich for giving me the opportunity to work in an incredible group and be part of many exciting projects. Throughout these four years, I learned a lot and received excellent support, encouragement and guidance for all my PhD work. I have to admit, it was a tough time, sometimes we had our differences, but in the end, I am indebted to your continuous support and for transforming me into a more confident person. I am always grateful to you for always believing in me and helping me move forward in making this memorable experience.

I take this opportunity to thank my thesis committee members, Prof. Dr. Dörthe M. Katschinski, Prof. Dr. Tiago Fleming Outeiro and Prof. Klaus-Armin Nave for their valuable guidance and critical input during my $\mathrm{PhD}$. In addition, many thanks to my examination board members, Prof. Dr. Susann Boretius, and Dr. Hauke Werner for taking the time to consider my work.

Moreover, I would like to express my gratitude to our collaborator, Prof. KlausArmin Nave for giving me the chance to work in his lab. I am thankful to Dr. Sandra Goebbels for critical insight and support during the cloning process. To Ulli, who was always full of positive energy and supported me during the tough time of cloning. You have always been a great help, listening to all my problems and motivating that I can do it. Constanze Depp for great help and support with the light-sheet microscopy work.

I would like to thank Prof. Dr. Susann Boretius for giving me the opportunity to learn and perform experiments in the DPZ. Special thanks to Nikoloz Sirmpilatze and Kristin Kötz for their continuous help during the fMRI experiments.

I would also like to acknowledge National University of Sciences and Technology (NUST) Pakistan for providing scholarship for my PhD under NUST Faculty Development Program Abroad 2014-15.

I am thankful to all the people in my whole journey, helped me more or less in their capacity and without their help and kindness I wouldn't have made it and 
this means a lot to me. I was extremely lucky to have such a nice working group. I learned a lot from all of you and was a great support and inspiration through all this time. Thanks to all the present and past members of our lab. I would like to acknowledge my colleagues Marina, Liane, Hana, Franziska, Laura, Annett, Sabine, and Daniela for all your help and support. To Victoria, thank you for excellent support and assistance with genotyping the entire time

To Dr. Rubina Dad, for your endless support during this entire time. I cannot thank you enough for all your time and help for reaching my goal.

Imam, Ohh "common on" and "still alive "You gave me these words as well. Thank you for everyday ongoing discussion and all the support. I will always remember this sentence "Don't do it, it's wrong" which you said on almost everything. I cannot recall any time, that I asked for help and you did not help me.

Anja, I do not know how to thank you for all your help and care. You have always been there for me watching over me as my elder sister.

Nadine, I really appreciate your support and help all the time. Especially, those words you have to do it now! And thinking about experiment more than me.

Hong, thank you for all your support, help, and positive energy. I hope I was able to contribute in making your life better.

Sahab, I am immensely grateful for your help and support all this time especially during thesis writing.

Jan, I think I do not have to Thank you.

Debia, thank you so much for help and although you are Indian and you are still fine

Agnes, Thank you so much your great help with work and the thesis. Moreover, listening to my boring stories.

Wiebke, Thank you for listening to my problems and giving advice regarding work and beyond.

Guilia and Barbara, I really appreciate your support and help in getting me started in the lab. Thank you so much such a lively and inspiring start. 
Ekrem, for such a lively environment. You were always a source of motivation and inspiration all the time.

My friends from Pakistan Dr. Suleman Riaz, Kashif Mehmood, Dr. Mustafeez Mujtaba Babar, Miriam Kathleen Gomez, Rabia Anwar, Nida ul Fatima, Saba Safdar, and Hussain Asif for their help and support in my journey to Germany.

I would like to thank all those people who did not help and support me during my work therefore motivating me to do work on my own.

Last but not the least, I am grateful to my family for their endless support and sacrifice more than words can express. I am thankful to my friends in Gottingen, Mohsin, Waqas, Zaheer, Umair, Rabia bhabi, Neelum, Sidra, Dr. Aamir Nadeem, and Ting because you already know and there are no words to express what you have done for me in the past years and counting!.

Lord Umer 

Dedicated

to my family 



\section{Table of Contents}

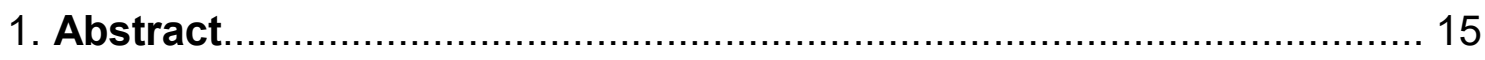

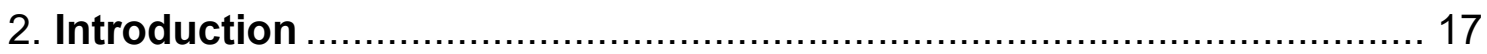

2.1 Aims and objectives of the present work..................................... 29

3. Chapter I. Establishment and preliminary characterization of hypoxia reporter mouse lines

3.1 Summary of chapter I

4. Chapter II - Visualizing functional hypoxia as primarily neuronal response to brain activity 35

4.1 Summary of chapter II 35

4.2 Chapter II - Visualizing functional hypoxia as primarily neuronal response to brain activity

4.3 Chapter II. Further experimental data on the CAG-CreERT2-ODD::R26RtdTomato line 66

5. Chapter III. Imaging hypoxic neurons with CaMKIla-based and inducible credependent tdTomato reporter mice ...................................................... 75

5.1 Summary of Chapter III............................................................ 75

5.2 Chapter III - Original publication............................................. 80

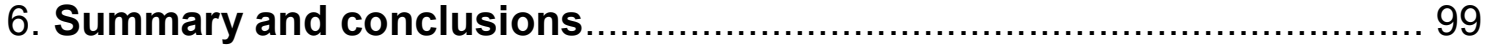

7. Outlook and follow-up studies ............................................ 105

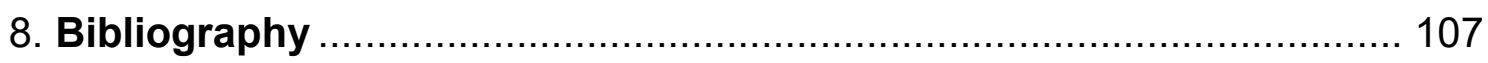

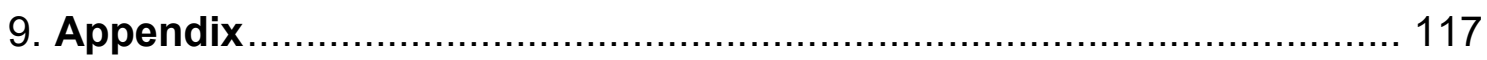

9.1 List of Abbreviations............................................................... 117

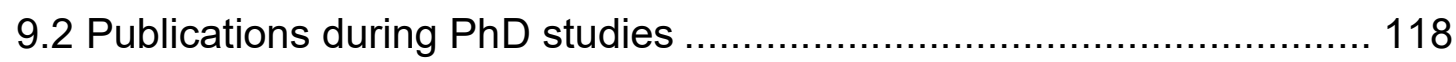





\section{Abstract}

Hypoxia is a critical regulator of the hypoxic response driving neurodevelopment, angiogenesis, hematopoiesis, and tissue regeneration. In the central nervous system, oxygen is a fundamental constituent of cellular homeostasis and is tightly controlled down to the single cells to maintain physiological functions. Under challenging conditions, slight alterations are detected and adaptive mechanisms are activated to adjust the homeostatic balance. In the brain, neuronal metabolic and network activity demands tightly controlled oxygen supply and any disruption results in detrimental outcomes. We therefore hypothesized that high neuronal activity would result in transient reduction of oxygen leading to physiological, 'functional' hypoxia followed by initiation of an adaptive gene response. A novel transgenic and robust approach, utilizing the oxygen-dependent degradation (ODD) domain of HIF-1 $\alpha$ fused to CreERT2 recombinase driven by CAG promoter was developed to report and label hypoxic cells upon breeding with tdTomato reporter mice. CAG-CreERT2-ODD::R26R-tdTomato transgenic mice allowed us to fate map hypoxic cells in the behaving brain under normoxia and hypoxia. We demonstrate an increase in the number of hypoxic neurons in the hippocampus upon complex motor cognitive challenge. Light-sheet microscopy whole-brain spatial mapping revealed a global distribution of hypoxic cells in the brain. Hypoxic cells were particularly abundant in the hippocampus and increased under inspiratory hypoxia. Hypoxic cell type characterization by immuno-labelling revealed that the major population of tdTomato+ cell were neurons followed by small subset of astrocytes, endothelial cells, oligodendrocytes and pericytes. Unexpectedly, the microglial population was devoid of tdTomato+ fluorescence in the brain. However, single-cell transcriptome analysis indicated comparable expression of ODD/tdTomato mRNA across cell-types in the hippocampus. These findings point to variable hypoxia tolerance and response of different cells in the brain. Furthermore, association of high neuronal activity with cognitive challenge was confirmed in new neuron-specific CaMKIla-Cre-ERT2-ODD::R26R-tdTomato transgenic mouse model. Hippocampal hypoxic neuron quantification results indicate an increase upon exposure to a challenging cognitive and motor task. Light-sheet microscopy analysis provided the spatial and temporal distribution of hypoxic neurons in the whole-brain. Taken together, these data provide evidence of the existence of physiological hypoxia in the brain under normoxia and its increase upon complex motor learning tasks. 



\section{Introduction}

\section{Brain homeostasis}

Over the course of evolution, oxygen has become an essential constituent of organism's survival, growth and development, demanding constant supply of energy used in metabolism to maintain normal cellular homeostasis (Hsia et al., 2013; Kump, 2008; Sato et al., 2011). In the central nervous system, especially in the brain, all cell types collectively play an important role to orchestrate responses to oxygen deprivation to monitor, control and maintain cellular homeostasis for regular brain functioning (Kriegstein et al., 2009; Roh et al., 2016). Small alterations, following challenging physiological or pathological conditions in brain homeostatic equilibrium are detected and responses are generated to restore homeostatic balance immediately (Dunwoodie, 2009). Molecular oxygen is one of the fundamental constituents of the cellular homeostatic machinery, necessary for energy production and metabolism via mitochondrial respiration and oxidative phosphorylation (Bélanger et al., 2011; Lewis et al., 1973). Under disease and adverse conditions, cells experience a hypoxic state resulting from reduced blood supply and altered cellular distribution. Hypoxia is a condition defined by significant reduction or drop of oxygen concentration in cells compared to normal ambient environmental oxygen concentrations $\left(20.9 \% \mathrm{O}_{2}\right)$ (Ainslie et al., 2010). There are several reports suggesting variable oxygen content and an innate oxygen threshold in different organs of the body. In mammals, arterial blood has the highest oxygen concentration after inhalation and following circulation. However, the oxygen concentration drops to its lowest levels in the deeper regions of the brain (Carreau et al., 2011; Ogoh et al., 2009).

Generally, oxygen deprived environments have been reported in pathological and physiological conditions. Pathologic hypoxia is considered a hallmark of tumors, inflammation and ischemic stroke conditions emanating from reduced blood and nutrient supply and leading to cell death and other dysfunctions (Eltzschig et al., 2011; Maxwell et al., 1997; Michiels, 2004; Ratcliffe, 2013). On the other hand, physiological hypoxia has proven to be an important driver of embryogenesis and development in the gestation period (Giaccia et al., 2004). Several lines of re- 
search suggest the existence of stem cell niches thriving in oxygen-poor environment in adult animals and considered essential for survival and maintenance (Mazumdar et al., 2010). Postnatally, under normal physiological conditions the stem cell niches give rise to new cells while in disease state by proliferation and differentiation meet the demands of modest functional recovery (Mohyeldin et al., 2010; Parmar et al., 2007).

In humans, the brain consumes around $20 \%$ of the entire metabolic energy (glucose and ATP), although in size the brain represents just $2 \%$ of the total body mass (Mergenthaler et al., 2013). Therefore, brain cells need a constant supply of nutrients and molecular oxygen to fuel their activities. Under disease and hypoxic challenges, the brain is the most susceptible due to an accumulation of free radicals and ionic imbalance (Bélanger et al., 2011). Therefore, several compensatory downstream mechanisms are triggered to maintain and restore normal cellular homeostasis (Brocato et al., 2014; Kenneth et al., 2008; Semenza, 2009).

One of the hallmarks of hypoxic stimuli is the activation of the hypoxia-inducible factors alpha (HIF- $\alpha$ ) family, a chief regulator of initiating the compensatory response to hypoxia in the cell (Wang et al., 1995a; Wang et al., 1995b). Hypoxiainducible factors belong to a family of transcription factors playing an important role in oxygen sensing, adaptation and maintaining homeostasis at cellular, organ and systemic levels (Kaelin et al., 2008). HIF- $\alpha$ activation and stabilization leads to the initiation of adaptive responses that support the cells to survive in hypoxic environments. Since its discovery in the 1990s, by Semenza and colleagues, HIF has been a center of hypoxia research covering the basic molecular and therapeutic strategies for cancer treatment (Semenza et al., 1991; Semenza et al., 1992).

\section{Structure of the HIF family}

Hypoxia-inducible factors form heterodimeric transcription factors consisting of HIF- $\alpha$ and HIF- $\beta$ subunits. These two subunits are part of the bHLH-PAS protein family, containing the basic helix-loop-helix (bHLH) motif that is required for binding to DNA in the nucleus (Wang et al., 1995a). HIF- $\alpha$ also contains PAS domains, which are essential for the dimerization process with HIF- $\beta$. HIF- $\alpha$ consists of two transactivation motifs which are termed as carboxy and amino terminal 
activation domains (CAD, NAD) (Pugh et al., 1997). The oxygen-dependent degradation (ODD) domain in NAD of HIF- $\alpha$ is an oxygen-labile, and therefore controls the stabilization and activity of hypoxia-inducible factors (Huang et al., 1996; Jiang et al., 1996; Wang \& Semenza, 1995b; Weidemann et al., 2008). In mammals, three different HIF- $\alpha$ isoforms are present: HIF-1 $\alpha$, HIF-2 $\alpha$ and HIF-3 $\alpha$ (Ema et al., 1997). HIF-1 $\beta$, also known as aryl hydrocarbon nuclear translocator (ARNT), is abundantly produced in the cell and is located in the cytosol and nucleus (Chilov et al., 1999; Wood et al., 1996). HIF-1a is ubiquitously expressed, and its activity is controlled by oxygen levels in the cells (Kamura et al., 2000; Wood et al., 1996). HIF-1 $\alpha$ consists of 826 amino acids whereas HIF- $2 \alpha$ consists of 870 amino acids and HIF-1 $\beta$ of 789 amino acids. HIFs have conserved proline residues in the ODD domains, which are vital for oxygen-dependent regulation by prolyl hydroxylase (PHDs) enzymes (Sutter et al., 2000). HIF-1 $\alpha$ contains conserved proline residues at positions (P402 and P564) and HIF-2 $\alpha$ at (P405 and P564) of the protein (Ivan et al., 2001). In contrast to less characterized HIF-3a (IPAS), the other two isoforms are $48 \%$ similar at amino acid level along with structural domains, therefore sharing the same HIF-1 $\beta$ subunit for dimerization, binding and transcriptional activation (Tian et al., 1997).

HIFs have variable expression levels throughout different organs and tissues and are modulated based on oxygen tension and environmental factors. HIF-1 $\beta$, a binding partner for the alpha subunits, is generally expressed in all cells under normoxic conditions. HIF-1 $\alpha$ expression has been reported in liver, kidney and brain at minimal levels, therefore implicating its role in maintaining the cellular homeostasis (Stroka et al., 2001). HIF-1a protein levels are regulated strictly and have a short half-life of around 5 min (Huang et al., 1998). The baseline expression of HIF-1a in the brain is upregulated upon hypoxia; it reaches the highest level at $5 \mathrm{~h}$, and takes $12 \mathrm{~h}$ to return to the normal level. Although the HIF- $\alpha$ isoforms share high structural similarity at protein level, the expression is variable in different cells across organs. HIF-1 $\alpha$ is expressed in most of the cells of the body and its expression in the brain has been reported in neurons, astrocytes, microglia, pericytes, endothelial and ependymal cells (Chávez et al., 2000; Ruscher et al., 1998). In contrast, HIF-2a expression is limited to certain tissues including interstitial cells in the kidney, parenchymal cells in the liver and endothelial cells. Therefore, it shows tissue specific expression and function (Tian et 
al., 1997; Wiesener et al., 1998). Like HIF-2a, HIF-3a expression is also tissue restricted with several isoforms reported due to alternative splicing (Makino et al., 2002). Some studies suggest HIF-3 $\alpha$ function as a negative modulator of HIF-1 and 2a-induced transcriptional changes (Gu et al., 1998; Makino et al., 2001). Other studies suggest sex related innate variation in the expression of HIF-1a under physiological and disease conditions, which may result in diverse responses to hypoxia stimuli (Zampino et al., 2006).

\section{HIF regulation}

HIF-1 $\alpha$ stabilization, activation and regulation are mainly controlled by oxygen levels in the cell. In an oxygen-enriched environment, HIF-1 $\alpha$ is regulated by posttranslational modifications (PTM) via hydroxylation of conserved proline residues in the ODD domain, carried out by PHDs (Myllyharju et al., 1997; Salceda et al., 1997). The functional activity of PHDs is regulated by oxygen levels in the cells along with other cofactors and act as an oxygen sensor. Upon hydroxylation of

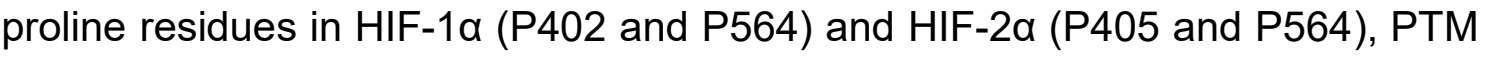
is recognized by the von Hippel-Lindau protein ( $p V H L)$, a recognition substrate of E3 ubiquitin ligase complex, which leads to ubiquitination and ultimately 26S proteasomal degradation (Maxwell et al., 1997; Yu et al., 2001). Under low oxygen conditions, oxygen required for hydroxylation by PHDs is missing, which results in HIF-1a stabilization (Chen et al., 2009; Huang et al., 1998). Consequently, HIF-1 $\alpha$ can enter the nucleus, where it binds to HIF-1 $\beta$, forming a dimer. Next, an active transcriptional complex is formed which binds to hypoxia response elements (HRE) of many target genes, attracts regulatory proteins including p300 and CBP for stimulation, and finally leading to downstream transcriptional activation (Arany et al., 1996; Brocato et al., 2014; Kallio et al., 1998).

HIF-1a stabilization and activity are also regulated by other PTMs via factor inhibiting enzymes (FIH), which belong to Fe (II)-dependent asparaginyl hydroxylase (Hewitson et al., 2002; Mahon et al., 2001). FIH enzymes also require oxygen for their activity and function by hydroxylating asparaginyl residues in HIF-1a at N803 and HIF-2 $\alpha$ at N847 in the C-terminal activation domain (CTAD) (Dengler et al., 2014). In the nucleus, CTAD plays an important role for recruiting the coactivators upon binding of the heterodimer to HRE of target genes, rendering CTAD essential for induction of transcriptional activity (Dann et al., 2002; Hu et 
al., 2007). By hydroxylating asparaginyl residues, FIH enzymes inhibit co-activator recruitment and halt the transcriptional activation (Lando et al., 2002). FIHmediated PTM function as an additional control on HIF-1 $\alpha$ stability and degradation in the cell. Arrest defective enzyme (ARD1) is also involved in the HIF-1a regulation by acetylating lysine residues in the ODD domain, however this modification is not controlled by the level of oxygen (Jeong et al., 2002).

PHDs act as one of the principle oxygen sensors and belong to the family of nonheme-dependent dioxygenases. These enzymes require oxygen, Fe (II), 2-oxoglutarate as a cofactor to carry out their catalytic activity and are primarily present in the cytoplasm (Berra et al., 2001; McNeill et al., 2002). PHDs' association with HIF- $\alpha$ was established a decade after the initial description of the hypoxiainducible factors. Under normoxic conditions, PHDs function by using oxygen to stimulate the hydroxylation of proline amino acids in the ODD domain of HIF- $\alpha$. Under hypoxic conditions, PHDs are unable to mark HIF-1a with a hydroxylation signal, necessary for $\mathrm{pVHL}$ recognition and recruitment for proteasomal degradation (Kaelin \& Ratcliffe, 2008; Marxsen et al., 2004; Urrutia et al., 2016). In literature, three common isoforms of PHDs are described: PHD1, PHD2 and PHD3, which are also termed as egg-laying defective nine homolog, EGLN2, EGLN1 and EGLN3 (Kaelin \& Ratcliffe, 2008). These isoforms are located in different cellular compartments where they display metabolite and tissue specific activities. PHD1 is reported to be specific for HIF-1 and $2 \alpha$ and has nuclear expression in many organs especially in the brain cells (Appelhoff et al., 2004; Bruick et al., 2001). PHD2 is expressed in both nucleus and cytoplasm and it is reported to have a specific role for HIF-1 $\alpha$ and is expressed at high levels in all tissues of the body (Epstein et al., 2001). PHD3 is found in the nucleus and cytoplasm, expressed in low amounts in all the tissues. Finally, another isoform was described as PHD4, which is a transmembrane protein involved in production and regulation of erythropoietin (EPO) (Gorres et al., 2010; Koivunen et al., 2007; Laitala et al., 2012). Overall, the HIF-VHL-PHD axis acts as oxygen sensor and plays an important role in regulating the HIF-mediated transcriptional response.

Accumulating evidence obtained from several studies suggest that oxygen-independent pathways are also involved in regulation of HIF-a (Koh et al., 2012). Some of the potential candidates from these studies include reactive oxygen 
species (ROS), sirtuins, nitric oxide (NO), and other metabolites (Sandau et al., 2001). HIF- $\alpha$ stabilization and activity are moderated by these factors but the exact functional mechanisms behind this regulation are not clear yet. Oxygen-independent HIF- $\alpha$ regulation controls transcriptional changes involved in cell metabolism, new vessel formation and homeostasis (Chun et al., 2002; Faton et al., 2013; Masoud et al., 2015).

HIF- $\alpha$ stabilization and activation leads to an initiation of many target pathways modulating erythropoiesis, angiogenesis, glycolysis, cell cycle control, anti-apoptotic factors and reconditioning metabolic switches to survive under hypoxic challenge (Dengler et al., 2014; Kaelin \& Ratcliffe, 2008). HIF- $\alpha$ activation during fetal development is crucial for normal growth and vascular system formation. Moreover, absence of any HIF- $\alpha$ isoform in mice leads to prenatal death of the embryos (lyer et al., 1998; Peng et al., 2000; Scortegagna et al., 2003). At present, HIF- $\alpha$ activated transcription is intensely investigated in adult mammals and various studies have confirmed several HIF-1 $\alpha$ and HIF-2 $\alpha$ common transcriptional targets, apart from novel cell and tissue specific target genes.

The primary targets of HIF- $\alpha$ transcriptional activation are a set of pathways controlling the oxygen homeostasis in the cells. So far, more than 100 direct target genes of HIF- $\alpha$ have been confirmed in different studies and the number is increasing every year (Khurana et al., 2013). Some of the common gene products regulated by HIF-1 $\alpha$ and HIF-2 $\alpha$ are VEGFA, BCL2, IL1 $\beta$, GLUT1 and carbonic anhydrase IX and XII (Chiche et al., 2009). HIF-1a activate exclusive gene products including nitric oxide production, LDHA, BNIP3, Wnt and Notch signaling. HIF-2 $\alpha$ specific targets include SOD2, EPO, OCT4, TGF $\alpha$ and Cyclin D1 (Blouin et al., 2004; Jelkmann, 2004; Lee et al., 2015). HIF-a-induced transcriptional activity leads to homeostatic changes by the expression of EPO, which is involved in the production of new red blood cells. This function is further facilitated by the VEGF, which controls new capillary formation in hypoxic regions, along with immediate discharge of nitric oxide (NO) resulting in vasodilation and increased blood supply in the affected area (Kenneth \& Rocha, 2008; Wang et al., 1995a).

Several studies suggest that HIF-2 $\alpha$ is mainly responsible for cellular production of EPO. In the kidney, interstitial cells are well known to produce EPO while in 
the brain neurons, glial cells, endothelial cells and pericytes are reported by ourselves and others to produce EPO (Chávez et al., 2000; Ehrenreich et al., 2004; Ehrenreich et al., 2007a; Engelhardt et al., 2015; Lewczuk et al., 2000; Sirén et al., 2001a; Sirén et al., 2001b; Sirén et al., 2005; Urrutia et al., 2016). During hypoxia, glucose transport is regulated by modifying the expression of glucose transporter 1 (GLUT-1) and additional regulators, crucial for glucose metabolism, in order to provide continuous supply of energy (Ainslie \& Ogoh, 2010; Sharp et al., 2004). HIF-3a has been suggested to play a role in modulating HIF-a-induced transcriptional activity by binding to HIF-1 $1 \beta$ and restoring the normal physiological levels in the cell (Ravenna et al., 2016). In summary, HIF- $\alpha$ activity controls a repertoire of target genes modulating the function of cells to repair, survive and compensate for hypoxic challenges.

\section{Oxygen sensing machinery at cellular and tissue level}

There are different homeostatic mechanisms in the body to detect and respond to a lack of oxygen. At tissue level, central and peripheral chemoreceptors perform an important role in detecting the reduced level of oxygen (O'Regan et al., 1982; Prabhakar et al., 2004). Central chemoreceptors are present in the medulla oblongata of the hindbrain region. These chemoreceptors detect changes in $\mathrm{pH}$ and carbon dioxide levels, initiating a recovery response by activating the respiratory center to regulate ventilation, heart rate and vasoconstriction to meet oxygen demand (Guyenet et al., 2015; Lahiri et al., 2007). Peripheral chemoreceptors are present on carotid and aortic bodies, where they mainly monitor the level of oxygen and carbon dioxide $\left(\mathrm{CO}_{2}\right)$ in the arterial blood (Kumar et al., 2012). The chemoreceptors contain special $\mathrm{K}+$ channels that are regulated by ROSs and $\mathrm{NADH}$. These radicals are generated due to fluctuation in the level of oxygen. Therefore, relaying fast response to adjust the oxygen supply under physiological and pathological conditions (Gourine, 2005; Lahiri et al., 2003).

Recently, pericytes were described as unique EPO producing cells among others in the brain by HIF-2 $\alpha$ regulated transcriptional activity (Urrutia et al., 2016). This new role of pericytes was demonstrated to sense low oxygen levels and activate the erythropoiesis process (Kisler et al., 2017; Urrutia et al., 2016). At subcellular level, many candidates have been reported to sense oxygen levels. Some of these players include PHDs, FIH (discussed above) and mitochondria involved in 
HIF- $\alpha$ activation and regulation (Waypa et al., 2016). Dioxygen is vital for the mitochondrial energy production, under hypoxic conditions the electron transport chain reaction is inhibited, which results in a rise in the levels of ROS. Redox changes in the cell also influence the action of PHDs and $\mathrm{FIH}$ and therefore elicit a similar response of HIF- $\alpha$ activation to trigger restorative mechanisms (Chandel et al., 2000). Overall, under reduced oxygen levels, mitochondria act as an oxygen sensors indirectly in the cell (Dunham-Snary et al., 2016).

\section{Exercise and learning-induced hypoxia in the brain}

Lack of physical activity and a sedentary lifestyle are the primary cause of diseases including obesity, cancer, stroke and heart malfunctions worldwide (Bhaskaran et al., 2014; Lee et al., 2012). A key complication observed across these diseases is the lack or insufficient supply of blood to the targeted areas, resulting in a shortage of oxygen and metabolites required for normal functioning (Chen et al., 2009; Marti et al., 2000). Under hypoxic ischemic conditions, HIFinduced transcriptional regulators trigger several downstream signaling pathways for cell survival and release of growth factors. Exercise-induced changes in the brain follow a similar pattern of response activation as observed during hypoxic events in the brain.

Several studies have reported an increased requirement of oxygen and glucose upon exercise to produce energy. Short bouts of exercise lead to HIF-1a stabilization and upregulation followed by short episodes of hypoxia in the cells (Kerr et al., 2011; Wu et al., 2019). Exercise leads to increased blood flow to the brain (Ogoh \& Ainslie, 2009; Querido et al., 2007). However, this increase is not happening in the whole-brain, but restricted to active areas. Numerous studies employing exercise and learning-paradigms in mice, have shown that upregulation of EPO and VEGF in the motor cortex, dentate gyrus of the hippocampus etc., results in high performance in cognitive behavioral tasks along with enhanced neurogenesis and angiogenesis (Adamcio et al., 2010; Berchtold et al., 2010; Bloor, 2005; Cao et al., 2004; Ehrenreich et al., 2007b; Ehrenreich et al., 2009; Favret et al., 2007; Jin et al., 2002; Sargin et al., 2011; van Praag et al., 1999; Wüstenberg et al., 2010). However, physical exercise and learning-induced alterations should be accounted as global phenomenon in the brain that affects several brain regions and not only those associated with motor movements. 
In the past decades, our understanding of the adult brain has changed from seeing it as a completely inflexible structure to a more modifiable in terms of formation of new neurons and blood vessels upon injury and exercise (Gould et al., 1999a; Gould et al., 1999b). Physical activity has been demonstrated to enhance learning and memory by generating and integrating new neurons into the hippocampal formations (Fabel et al., 2008). In the adult animals, new cells are generated in the dentate gyrus (DG) upon running and incorporated into the circuitry (van Praag et al., 1999). Following these interesting discoveries, several groups reported the effects of different housing and environmental enrichment schemes in mice, leading to improved cognition and memory resulting from neurogenesis and angiogenesis in comparison to inactive mice (Jankowsky et al., 2005; Liu et al., 2018; McKenzie et al., 2014; Meijer et al., 2014; van Praag et al., 2005). It is a common understanding that physical exercise improves cognitive functions in all populations irrespective of age (Cotman et al., 2007; Hillman et al., 2008).

Recently, Wu et al. 2019 showed exercise-induced physiological hypoxia in a peripheral organ, by using HIF-1a luciferase reporter mice. In this study, the authors initially checked the normal physiological hypoxia in the body by pimonidazole hydrochloride, followed by using ODD luciferase reporter mice under different exercise paradigms (Safran et al., 2006). Interestingly they reported an upregulation in the level of HIF-1 $\alpha$ and hypoxia in the small intestine upon physical activity (Wu et al., 2019).

In summary, several studies provide adequate proof of physiological hypoxia as an important driver of growth, survival, division and stemness of precursor cells (Adamcio et al., 2010; Hassouna et al., 2016; Mazumdar et al., 2010; Mohyeldin et al., 2010). Accumulating evidence from our lab and others suggest that physical exercise lead to a hypoxic state at the tissue or cellular level due to exceeding oxygen, glucose and metabolite demand in the respective active areas (Adamcio et al., 2008; Adamcio et al., 2010; Kerr \& Swain, 2011). Exercise-induced hypoxia in the brain and muscles leads to an activation of downstream pathways, comprising enhanced oxygen supply and metabolic switches to maintain and restore normal cellular homeostasis (Cao et al., 2004; El-Kordi et al., 2009; Goodall et al., 2014; Hara et al., 1990; Ogoh \& Ainslie, 2009; Wu et al., 2019). 


\section{Oxygen sensing tools in the hypoxia-imaging field}

Over the last few decades, there has been a lot of advancement in the field of hypoxia sensing due to the indispensable role of oxygen in cellular and developmental functions. Multiple techniques and methods have been described to study, visualize, and label the hypoxic machinery at cellular and sub-cellular resolution. These techniques allow investigators to monitor and examine oxygen tension in vitro and in vivo, in settings ranging from normal physiological to pathological conditions (Erapaneedi et al., 2016; Esipova et al., 2019; Goldman et al., 2011; Lidsky et al., 2018; Misra et al., 2016; Safran et al., 2006; Spencer et al., 2014). However, these settings are limited due to the requirements of a complex and high throughput machinery followed by invasive and toxic procedures (Papkovsky et al., 2018). Cellular resolution and indirect measurement of oxygen levels further contribute to the limited success of current oxygen sensing technologies.

Some of the well-known hypoxia imaging techniques employ dye-based biosensors or genetically modified fluorescent proteins for detection of oxygen tension in the cells. Dye-based oxygen biosensors have been characterized over the past decade, showing a great potential in detecting oxygen levels (Mirabello et al., 2018; Sandhu et al., 2017; Sato et al., 2011). Frequent use of these dyes is hampered by complex experimental conditions and reports of cell toxicities (Papkovsky \& Dmitriev, 2018). Fluorescent protein-based hypoxia detection methods are commonly used, due to genetic encoding and expression at the cellular and tissue level (Feil et al., 1997). The intrinsic activation, expression and labelling of specific cellular compartments make these fluorescent proteins, with tunable spectral visualization, feasible for in vivo models (Branda et al., 2004; Metzger et al., 2001). Most of these oxygen sensors are either equipped with a hypoxia responsive promoter or carry the ODD domain of HIF-1a placed above the cell type specific promoters (Erapaneedi et al., 2016; Kimura et al., 2015). These methods provide an indirect measure of oxygen undersupply in the cells visualized later at cellular level due to the presence of fluorescent proteins under the fluorescence microscope (Danhier et al., 2015; Misra et al., 2016; Yudkoff et al., 1988). 
To date, several transgenic mice have been described in the literature, to study hypoxia under in vivo conditions. Initially, Rosa26 ODD-Luc/+ mice based on the combination of the firefly luciferase sequence with the ODD domain of HIF-1a have been used to study tissue hypoxia. Although these mice report HIF stabilization, ease of use is hampered by the essential requirement of luciferin for signal detection, along with oxygen and adenosine triphosphate (ATP) (Safran et al., 2006). Recently, a new fluorescent protein, UnaG, was described, allowing the detection of hypoxic and re-oxygenated states. This protein is genetically encoded and works by detecting and labelling activated hypoxia-inducible factor without oxygen requirement for detection in the cell (Erapaneedi et al., 2016). However, authors tested the working efficiency of the method only in cell lines and tumor tissues. Another hypoxia-reporter mouse strain was described in the context of fate mapping progenitor like cardiomyocytes in the heart tissue (Kimura et al., 2015; Nakada et al., 2016). CAG-CreERT2-ODD::R26R-tdTomato mice were based on genetically encoded tdTomato fluorescence-based detection of all hypoxic cells. Taken together, all the hypoxia detecting methods have widely studied cellular hypoxia is in peripheral and tumor tissue. However, the area of brain cellular hypoxia is neglected and is not investigated in detail.

\section{$\mathrm{Ca}^{2+1}$ calmodulin-dependent protein kinase II alpha (CaMKIla) promoter ex- pression}

Since its discovery, CaMKIla, has been implicated in several important physiological functions covering learning, memory and synaptic plasticity (Lisman et al., 2002; Schulman et al., 1978). Calcium/calmodulin-dependent protein kinases (CaM) are part of serine/threonine (Ser/Thr) protein kinases and are intensely investigated in the brain due to elevated expression levels in the adult forebrain (Minichiello et al., 1999). CaMKIla, is abundantly expressed in pyramidal neurons, especially at the postsynaptic densities (PSDs) and steadily contribute to learning and memory (Erondu et al., 1985; Mayford et al., 1996; Wang et al., 2013). Calmodulins are best characterized as calcium sensing proteins that are activated by second messenger binding $\left(\mathrm{Ca}^{2+}\right)$. Activated calcium-bound calmodulin (CaM) regulate and permanently activate calcium/calmodulins ( $\left.\mathrm{Ca}^{2+} / \mathrm{CaM}\right)$ dependent protein kinase (CaMKIIa) and initiate many downstream targets involved in long-term potentiation (LTP) (Chin et al., 2000; Erondu \& Kennedy, 
1985). Due to brain-specific neuronal expression and validation in several transgenic mice, CaMKIla promoter have been employed for the generation of transgenic mice previously and was also used for generation of neuron-specific hypoxia reporter transgenic mice (Mayford et al., 1996; Tsien et al., 1996; Wang et al., 2013).

\section{Transcriptome analysis of hypoxic cells in the brain}

Hypoxic exposure leads to alteration in the transcriptional profile of different cell types in the brain. Previous RNA sequencing studies on tumor tissues have reported deregulation in several pathways associated with metastasis, angiogenesis and mainly upregulation of the HIF-1a for survival under reduced oxygen and energy metabolite environment (Harris et al., 2018; Hochgerner et al., 2018; Louie et al., 2010). Single cell transcriptome analysis during development and adult age have revealed great diversity and heterogeneity among different tissues across cell populations (Butler et al., 2018; Stuart et al., 2019). In the present study, single cell RNA sequencing of hippocampal cells treated with hypoxic and normoxic conditions were investigated to delineate the effects of hypoxia. 


\subsection{Aims and objectives of the present work}

In the introduction, I discussed how cellular homeostasis is maintained and tightly modulated by adequate oxygen, metabolic and energy support. This regulation is essential for all physiological cellular activities. In the central nervous system, especially in the brain, cellular homeostatic process particularly the oxygen level is inflexibly coordinated from regional levels down to neuronal connections at the synapses (Sharp \& Bernaudin, 2004). Furthermore, as I highlighted that a modest number of tools have been developed to study the basic hypoxia machinery and to visualize the labelled cells undergoing hypoxia in vitro and in vivo (Erapaneedi et al., 2016; Esipova et al., 2019; Goldman et al., 2011; Lidsky et al., 2018; Misra et al., 2016; Safran et al., 2006; Spencer et al., 2014). Despite the fact that these techniques provide new ways to monitor and study oxygen in different tissues, the exact oxygen concentration and hypoxia sensing machinery in different brain cell types is poorly understood, both under normal and disease conditions. Therefore, the aim of the present thesis is to gain more insight into the brain's hypoxic environment and its association with exercise, learning and memory.

Our group has previously shown increased long-term potentiation (LTP) and improved performance in cognitive and learning tasks in adult mice upon treatment with EPO (Adamcio et al., 2008; El-Kordi et al., 2009). The underlying mechanism of improved performance in learning tasks was thoroughly uncovered by Hassouna et al. 2016. The authors have shown a $20 \%$ increase in the number of cornu ammonis (CA) neurons in the pyramidal layer of the hippocampus within only 3 weeks of EPO administration along with an increase in learning and cognitive performance (Hassouna et al., 2016). It was postulated that EPO administration influenced the neuronal precursor cells by driving the differentiation and resulting in significant increase in mature neurons in the hippocampus. On the other hand, other groups have reported that exercise, cognitive challenge and learning tasks demand a high metabolic and oxygen content along with more neurons for a better performance (Berchtold et al., 2010; Kerr \& Swain, 2011; Wu et al., 2019). Taken together, an increase in the number of pyramidal neurons and enhanced cognitive flexibility could be explained by HIF-1 $\alpha$ stabilization under transient episodes of functional hypoxia that facilitate the proliferation and 
better performance in learning tasks. To understand and investigate our hypothesis that challenging learning leads to hypoxia in the brain, a new oxygen sensing transgenic mouse model expressing inducible cre-recombinase fused to ODD domain of HIF-1a was developed. The mice express a ubiquitous CAG promoter to fate map all hypoxic cells playing a crucial part in learning and memory (Kimura et al., 2015). The recruitment and employment of these new hypoxia reporter mice aims to develop a better insight into the hypoxic state in the brain and is fundamental to study the effect of exercise and motor-learning-induced hypoxia.

Chapter I comprises preliminary work performed to generate hypoxia reporter mice. It contains the summary of different problems faced during generation of new transgenic mice and as well as basic experiments performed to understand oxygen sensing machinery under different experimental paradigms.

Chapter II encompasses results from hypoxia-reporter mice, carrying a ubiquitous, CAG promoter to explore the brain's regional oxygen tension differences in all cell types. This transgenic mouse model will further provide a deeper understanding of the different hypoxic cellular populations in the brain.

As we noticed that, the neuronal population is most susceptible to physiological and pathological hypoxic challenges in the brain, we aimed at developing a new neuron-specific hypoxia reporter mouse in chapter III. CaMKIla-CreERT2ODD::R26R-tdTomato mice, would allow studying the effects of exercise and learning in particular in the neuronal population in the brain regardless of other cell populations. CaMKIla expressing hypoxic neurons will provide a better understanding of neuronal oxygen sensitivity and global distribution in the brain. 


\section{Chapter I. Establishment and preliminary characterization of hypoxia reporter mouse lines}

\subsection{Summary of chapter I}

To address the issue of hypoxia labelling in the brain, new hypoxia reporter mice were generated for basic research as described in chapter II and III. The global hypoxia reporter mouse has been previously described (Kimura et al., 2015). To produce hypoxia reporter mice in our lab, methods comprising cloning, sequencing quality control and microinjection of the CAG-CreERT2-ODDpA plasmid were performed. In the following, the summary of different hurdles with respect to the generation of these transgenic mice as well as further experiments to check the activity of the oxygen sensing machinery in the mice brain.

After generation, founder mice were checked for presence of the transgene in the genome by genotyping PCR. Subsequently, potential founder mice were selected and were further bred with C57BL/6N and tdTomato reporter mice. Initially, F1 litters carrying the transgene were selected for validation of the hypoxia sensing machinery. Mice were checked under hypoxic $\left(6 \% \mathrm{O}_{2}\right)$ and normoxic $\left(21 \% \mathrm{O}_{2}\right)$ conditions along with tamoxifen injections. Unfortunately, we did not observe any expression of tdTomato reporter in this experiment except single mouse. Although the transgene was present in the genome upon checking via genotyping PCR, the labelling of tdTomato fluorescent protein was not found in the brain. To evaluate this issue, we screened the literature for potential complications associated with transgenic mice. In the original publication, the authors also report unexpected silencing of the transgene in the transgenic mice. Therefore, the transgenic mice were kept on a mixed genetic background instead of a pure C57BL/6 background.

After careful screening of the literature with regard to problems associated with transgenic mice, we speculated that the absence of the transgene expression could be due to epigenetic gene silencing. There are many reports in the literature, linking the presence of bacterial sequence with transgene silencing in the mouse genome (Chen et al., 2004; McEachern, 2012; Suzuki et al., 2006). The CAG-CreERT2-ODDpA plasmid also contains a bacterial backbone, comprising of ampicillin selection cassette (Figure 1a). In order to avoid this potential problem 
the bacterial sequence was removed by restriction digestion, for subsequent generation of transgenic mice. The plasmid was sequenced again and its linear form was used for generation of new transgenic mice by microinjection (Figure 1b). Subsequently, a long process for breeding and selection of suitable mice for experiments was conducted.

In a similar scheme, as described above, founder mice carrying the transgene were selected and bred with C57BL/6N and tdTomato reporter mice. F1 litters containing the CAG-CreERT2-ODD::Rosa26-tdTomato transgene were evaluated under hypoxic and normoxic conditions to test for oxygen sensitivity of the transgene using the experimental scheme described in the original publication. Remarkably, we observed a sparse population of tdTomato+ hypoxic cells under normoxic conditions and a major increase in the number of hypoxic cells upon application of exogenous hypoxia. In order to generate neuron-specific hypoxia sensitive mice the CAG promoter was replaced with (Figures 1a and c) CaMKIla promoter to obtain CaMKIla-CreERT2-ODD expressing transgenic mice (Figure 1d).

Ligand-independent labelling of cells was checked in CAG-CreERT2ODD::Rosa26 reporter mice ( 4 and 56 weeks old) as shown in figure $2 a$ and $b$. We observed only a few tdTomato+ cells in 4 weeks old mice compared to 56 weeks of age. In older mice, the number of non-specific tdTomato+ cells accumulated over time and therefore produced considerable background signal. These experiments were essential for the characterization and evaluation of the working efficiency of this reporter system. Collectively, we can infer from these results that CAG-CreERT2-ODD::Rosa26 reporter mice are useful to study hy- 
poxia in younger age. In contrast, we observed spontaneous cre activity and accumulation of tdTomato+ cells upon aging leads to non-specific background signal, which could hamper the data interpretation.
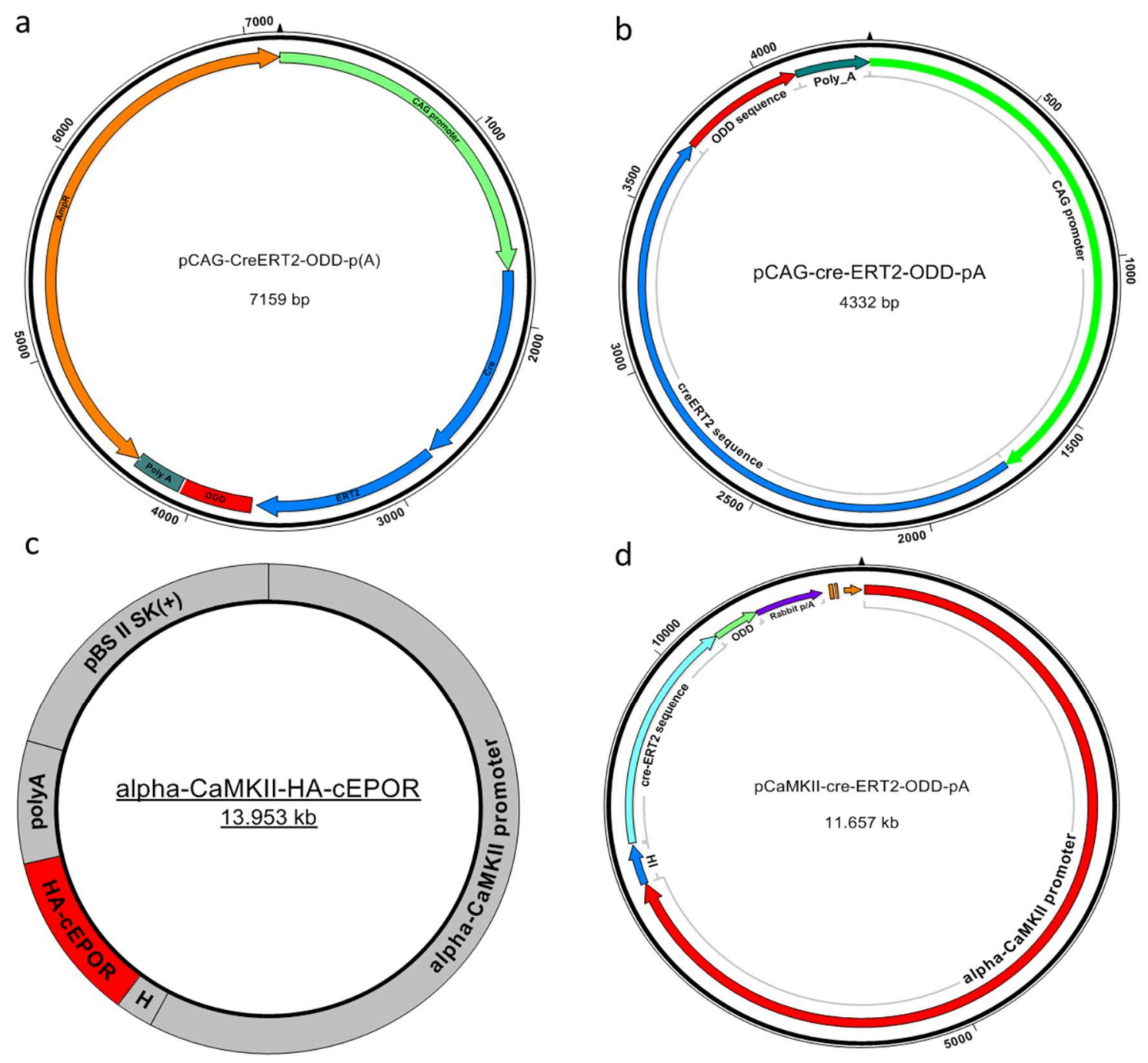

Figure 1. Schematic representation of the hypoxia reporting constructs. Original CAG-CreERT2-ODDpA vector is shown in figure 1a. CAG promoter is driving the expression of inducible cre-recombinase fused with ODD domain. This vector also contains ampicillin resistance-cassette required for antibiotic selection during cloning procedure. Figure $1 \mathrm{~b}$ represents the modified CAG-CreERT2-ODDpA vector. For microinjection process, vector was linearized and ampicillin sequence was removed to avoid the transgene silencing. CaMKIla promoter was derived from alpha-CaMKIIHA-cEPOR vector (figure 3b). The CAG promoter was removed from CAG-CreERT2ODDpA vector (figure 1a) and CaMKIla promoter was inserted in the CreERT2ODDpA vector backbone to assemble CaMKIla-CreERT2-ODDpA (figure 1d). 


\section{No tamoxifen}

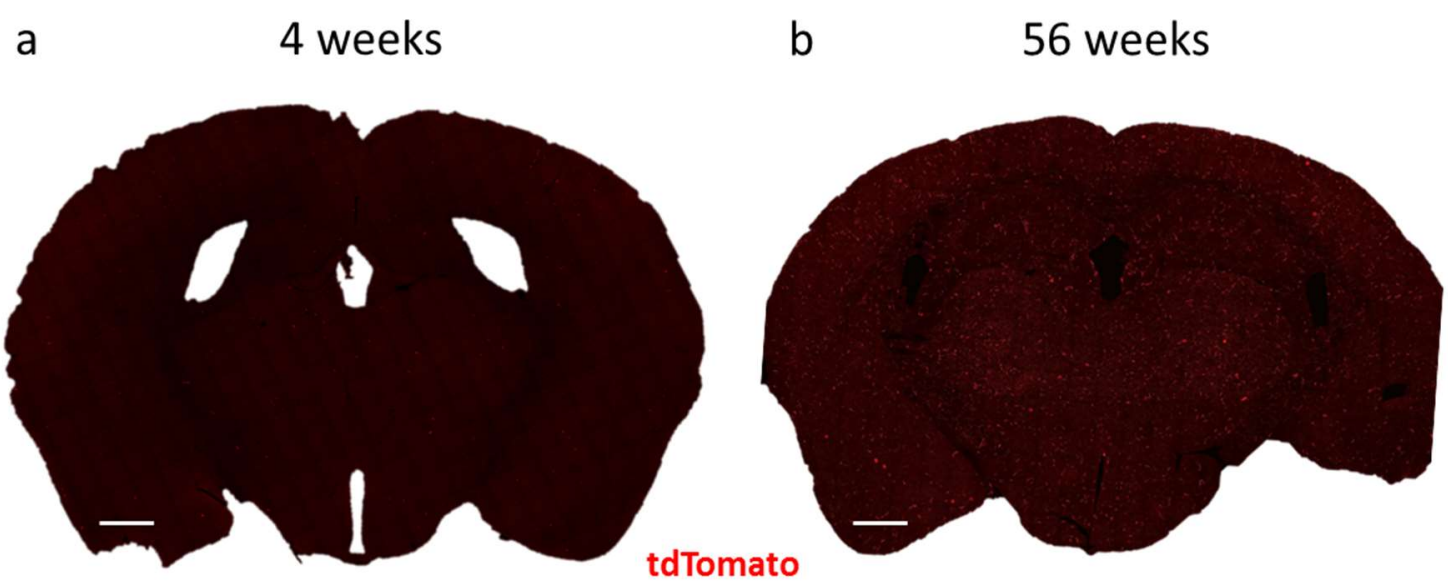

Figure 2. Overview coronal image of a 4 and 56 weeks old non-treated CAGCreERT2-ODD::R26R-tdTomato mice. (a) Control mice without any treatment were perfused and checked under the confocal microscope. Ligand (tamoxifen)independent labelling of cells with tdTomato (red) was observed throughout the whole-brain in the old age mice. Scale bar represents $500 \mu \mathrm{m}$. 


\section{Chapter II - Visualizing functional hypoxia as primarily neu- ronal response to brain activity}

\subsection{Summary of chapter II}

In the brain, cellular homeostasis is tightly controlled, down to single cells to maintain physiological functions. Under challenging conditions, minor alterations are detected and adaptive mechanisms are activated to adjust the homeostatic balance (Sharp \& Bernaudin, 2004). Oxygen is one of the fundamental constituents of cellular homeostasis and is central in cellular metabolic activity via energy production.

In recent years, given the central role of oxygen, only a modest number of tools have been developed to study the basic hypoxia machinery and to image and label the hypoxic cells in in vitro and in vivo conditions (Erapaneedi et al., 2016; Esipova et al., 2019; Goldman et al., 2011; Lidsky et al., 2018; Misra et al., 2016; Safran et al., 2006; Spencer et al., 2014). Although these techniques provide new approaches to monitor and study oxygen tension in cells, common use of these oxygen-sensing tools is hindered due to the requirement of complex and high throughput machinery (Papkovsky \& Dmitriev, 2018). In addition, most of these techniques involve invasive and toxic procedures for oxygen sensing and report an indirect measure of oxygen levels in the cells, which may contribute to the limited output. Unfortunately, most of previous work focuses on peripheral organs and the role of HIF-1a with respect to brain cell type specific hypoxia response has not been thoroughly investigated. In 2015, Kimura et al. published a hypoxiareporter mouse strain expressing a ubiquitous CAG promoter in conjunction with inducible cre-recombinase fused to the ODD domain of HIF-1 $1 \alpha$. These mice allow the labelling of all hypoxic cells upon tamoxifen administration under physiological and pathological conditions. In order to investigate the role of hypoxia in different cell populations in the brain, we aimed to generate CAG-CreERT2-ODD transgenic mice as described before (Kimura et al., 2015).

Our group has previously demonstrated that EPO induces cognitive improvement under health and disease in human and mice in several behavioral paradigms (Adamcio et al., 2008; Adamcio et al., 2010; Ehrenreich et al., 2004; Ehrenreich et al., 2007a; Ehrenreich et al., 2007b; Ehrenreich et al., 2009; El-Kordi et al., 
2009; Lewczuk et al., 2000; Sargin et al., 2011; Sirén et al., 2001a; Sirén et al., 2001b; Sirén et al., 2005; Wüstenberg et al., 2010). Subsequently, the underlying mechanisms resulting in cognitive improvement, were systematically uncovered by revealing an increase in the number of pyramidal neurons in the hippocampus of EPO treated mice (Hassouna et al., 2016). Considerable research from many investigators has reported an enhanced requirement of oxygen and glucose for energy production in the active brain region during exercise, learning and memory tasks (Kerr \& Swain, 2011; van Praag et al., 2005; Wu et al., 2019). These homeostatic alterations upregulate HIF-1a thereby activating hypoxia response pathways. Hence, we proposed that an increase in the number of pyramidal neurons and enhanced cognitive flexibility could result from short episodes of functional hypoxia in the hippocampus of active animals. Therefore, we hypothesized that so-called functional hypoxia is the driving force behind the increase in neurons, which further facilitates the proliferation of existing cells and cognitive improvement. We aimed to test the hypothesis exercise and learning leads to hypoxia in the brain of mice. Therefore, hypoxia reporter mice carrying CAGCreERT2-ODD transgene were generated allowing the labelling of hypoxic cells upon HIF-1a stabilization.

In preliminary experiments, CAG-CreERT2-ODD transgenic mice were crossbred with R26R-tdTomato reporter mice to permanently label hypoxic cells. Working efficiency of the reporter system and tracing of hypoxic cells was validated by application of exogenous hypoxia in the CAG-CreERT2-ODD::R26R-tdTomato mice. Negligible background leakiness was observed in the non-tamoxifen treated mice and did not influence the data interpretation. In this study, for the first time by means of the CAG-CreERT2-ODD::R26R-tdTomato transgenic mice, it was shown that a sparse population of hypoxic cells labeled with tdTomato fluorescent signal are present under normal physiological conditions and this is in agreement with previous immunoblot reports (Stroka et al., 2001). Overall, the number of tdTomato+ hypoxic cells increase upon the application of exogenous hypoxia.

Tamoxifen and oxygen dose-response curve: $A$ tamoxifen dose-response curve was established in CAG-CreERT2-ODD::R26R-tdTomato transgenic mice demonstrating a rise in the amount of hypoxic cells in the brain upon tamoxifen 
$(100 \mathrm{mg} / \mathrm{kg} / \mathrm{d})$ titration $(1 \mathrm{x}, 3 \mathrm{x}$ and $5 \mathrm{x}$ injections). Targeted quantification of hippocampal neurons rendered the 5-tamoxifen injection paradigm suitable for modest labelling in the brain. Next, we tested the effect of severity of hypoxic challenges $\left(20.9 \%, 15 \%, 12 \%, 10 \%, 8 \%\right.$ and $\left.6 \% \mathrm{O}_{2}\right)$ in CAG-CreERT2-ODD::R26RtdTomato transgenic mice and quantified the number of hypoxic neurons in the hippocampus. The results of oxygen-dose-response analysis indicated a dramatic expansion in the number of hypoxic neurons (highest in $6 \% \mathrm{O}_{2}$ ) upon decrease in oxygen concentration in comparison to ambient oxygen levels $(20.9 \%$ $\mathrm{O}_{2}$ ). The oxygen-dose-response curve analysis elegantly demonstrates the sensitivity of the oxygen sensing machinery and validates the hypoxia response in the CAG-CreERT2-ODD::R26R-tdTomato transgenic mice. Furthermore, under normal physiological conditions long-term tdTomato labelling efficiency was investigated in the mice over the period of 1 month. Hypoxic neuron quantification exhibited lasting persistence of the tdTomato signal in the brain. In summary, these results suggest oxygen-dependent labelling of hypoxic cells in the brain of CAG-CreERT2-ODD::R26R-tdTomato transgenic mice under different experimental conditions thereby highlighting the potential for long-term fate mapping experiments.

Cellular characterization of hypoxic cells: After confirming the oxygen-dependent labelling of hypoxic cells in the brain, we explored the identity of tdTomato fluorescent-labelled cells in the brain. Hypoxic cells were characterized by immunohistochemistry, using cell type specific markers including NeuN for mature neurons, IBA1 for microglia, $\mathrm{S} 100 \beta$ for mature astrocytes, Olig2 for oligodendrocytes, CD13 for pericytes and lectin for endothelial cells along with the blood vessels. Stained sections were checked under the confocal microscope for colocalization with tdTomato+ cells. Surprisingly, microglial cells were considered non-responsive to hypoxia since no co-labelling of IBA-1 with tdTomato fluorescence signal was detected in the whole-brain. This finding raised the question of variability in expression of CAG-CreERT2-ODD-pA transgene in different brain cell types. In order to understand and clarify the diverse expression of CAGCreERT2-ODD-pA transgene in different cell types as well as to eliminate the possibility of absence of transgene expression in microglia, single cell transcriptome analysis of hippocampi from hypoxia and normoxia treated mice was performed. Overall, data analysis revealed a comparable expression of both 
transgenes (ODD, tdTomato) in all cell types of the hippocampus along with upregulation of hypoxia regulated genes (Cao et al., 2004). Presence of both transgenes (ODD, tdTomato) in microglial population confirms the expression at mRNA level. However, absence of corresponding tdTomato protein signal in histology could be explained by cell type specific hypoxia tolerance. During cell type specific hypoxia tolerance analysis, we made the startling discovery that the gene encoding hexokinase2 (Hk2) revealed a prominent expression in microglia under hypoxic and normoxic conditions. Hk2 (glycolytic enzyme) expression abundance in microglia may provide resistance and tolerance to hypoxic stimuli as noticed in tumor cells (DeWaal et al., 2018; Wolf et al., 2011).

Learning-induced hypoxia: Next, we tested our hypothesis that exercise and learning leads to functional hypoxia in the brain of CAG-CreERT2-ODD::R26RtdTomato transgenic mice. In this experiment, 4 and 24 weeks old mice (both sexes) were included and divided into four groups. In the treatment paradigm, mice received a daily tamoxifen injection for 5 days. Mice undergoing hypoxia were exposed to $6 \% \mathrm{O}_{2}$ for $6 \mathrm{~h}$ on 5 consecutive days. Mice undergoing voluntary wheel-running were exposed to complex running wheels for 5 days and mice undergoing normoxia were kept under normal housing conditions. Additionally, a group of control mice were administered with a daily injection of oil for 5 days, to control for non-specific tdTomato labelling in the brain. Mice were sacrificed on day 8 , and the brains were collected for histological examination and image acquisition under confocal microscope. Targeted quantification of tdTomato+ neurons in cornu ammonis layer (CA1, CA3) and dentate gyrus (DG) of the hippocampus was performed in all groups. Hypoxia treated mice revealed highest increase in hypoxic neurons in the hippocampus. Interestingly, quantification results revealed a modest increase in the number of hypoxic neurons in mice that underwent voluntary wheel running in comparison to control mice under normoxia, pointing to an exercise and learning-induced increase in the number of hypoxic neurons. This increase can be explained by high oxygen and metabolite demand in the active areas, leading to a transient state of functional hypoxia in the cells. In particular, CAG-CreERT2-ODD::R26R-tdTomato female mice revealed a somewhat higher number of hypoxic neurons in response to hypoxia 
and complex running wheel exposure as compared to male mice, suggesting sex specific difference in the HIF-1 $\alpha$ activation machinery (Zampino et al., 2006).

Light-sheet microscopy: We employed modern imaging technology to map the overall distribution of hypoxic cells in the whole-brain by light-sheet microscopy (LSM). The 3-dimensional presentation of tdTomato+ hypoxic cells was checked under hypoxia, complex running wheel, normoxia and in placebo treated mice, revealing a global distribution in the whole-brain. Cortical and hippocampal total hypoxic cell quantification revealed an increase in hypoxia treated mice in comparison to the normoxia control group validating the histological quantification. Sparse population of hypoxic cells were also observed without tamoxifen administration, which could be explained by non-specific tdTomato antibody immunoreactivity and spontaneous cre-recombinase activity. CRW exposed mice in the LSM experiment showed a similar increase in total number of hypoxic cells in the hippocampus, as observed with histology quantification in comparison to normoxia mice. Here, the increase in hypoxic cells in CRW mice, as demonstrated by histology and LSM, strongly suggests high neuronal activity in the hippocampus in response to exercise and learning. These results further support and confirm our hypothesis, that exercise and learning lead to a metabolically challenging environment in brain cells which results in transient episodes of hypoxia (Wu et al., 2019).

In conclusion, CAG-CreERT2-ODD::R26R-tdTomato is an invaluable hypoxia sensing tool to study reduced oxygen levels in different cell types in mice under normal physiological and pathological conditions. By using hypoxia-sensing mice, we provide evidence that exercise and learning activity leads to a hypoxic and metabolically stimulating environment in the brain, especially in neurons, leading to functional hypoxia. In the future, these mice will be a vital tool to detect and study hypoxia in the brain. 


\section{Original publication}

Butt, U.J*., Steixner-Kumar, A.A*., Depp, C*., Sun, T., Hassouna, I., Wüstefeld, L., Arinrad, S., Zillmann, M.R., Schopf, N., Garcia-Agudo, L.F., Mohrmann, L., Bode, U., Ronnenberg, A., Hindermann, M., Goebbels, S., Bonn, S., Katschinski, D.M., Miskowiak, K.W., Nave, K.A., \& Ehrenreich, H. " Hippocampal neurons respond to brain activity with functional hypoxia." Molecular Psychiatry (2021): 118.

* Shared first authorship.

\section{Personal contributions}

I was responsible for the planning and development of the ODD project under supervision of Prof. Dr. Dr. Hannelore Ehrenreich. Initially, I participated and completed the construction of CAG-CreERT2-ODD-pA plasmid and developed the CAG-CreERT2-ODD transgenic mice. Additionally, I carried out the basic characterization and validation of hypoxia reporter mice. I performed the hypoxia, normoxia, and CRW experiments and provided the results for the research article. For functional characterization, I performed the experiments and provided the samples for LSM. Images and videos were prepared by our collaborator CD. Similarly, I prepared the samples and conducted the hypoxia experiment for single cell RNA sequencing. Data was analyzed by my colleague AS. I contributed to writing of the paper, preparation of figures, materials and methods. Together with my supervisor and the first authors, I finalized the research article for publication. 


\title{
4.2 Chapter II - Visualizing functional hypoxia as primarily neuronal re- sponse to brain activity
}

Molecular Psychiatry

https://doi.org/10.1038/s41380-020-00988-w

ARTICLE

\section{Hippocampal neurons respond to brain activity with functional hypoxia}

\begin{abstract}
Umer Javed Butt $\oplus^{1} \cdot$ Agnes A. Steixner-Kumar $\odot^{1} \cdot$ Constanze Depp $\odot^{2} \cdot$ Ting Sun $^{2,3} \cdot$ Imam Hassouna $^{1}$.

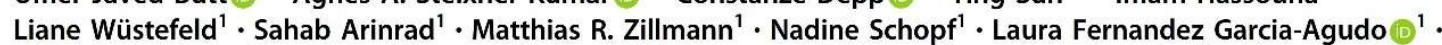
Leonie Mohrmann ${ }^{1} \cdot$ Ulli Bode ${ }^{2} \cdot$ Anja Ronnenberg $^{1} \cdot$ Martin Hindermann ${ }^{1} \cdot$ Sandra Goebbels $^{2} \cdot$ Stefan Bonn $^{3}$.

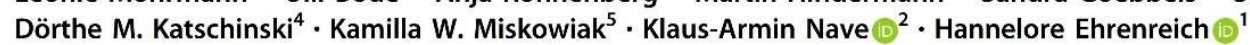

Received: 2 June 2020 / Revised: 24 November 2020 / Accepted: 4 December 2020

(c) The Author(s) 2021. This article is published with open access

\section{Abstract}

Physical activity and cognitive challenge are established non-invasive methods to induce comprehensive brain activation and thereby improve global brain function including mood and emotional well-being in healthy subjects and in patients. However, the mechanisms underlying this experimental and clinical observation and broadly exploited therapeutic tool are still widely obscure. Here we show in the behaving brain that physiological (endogenous) hypoxia is likely a respective lead mechanism, regulating hippocampal plasticity via adaptive gene expression. A refined transgenic approach in mice, utilizing the oxygen-dependent degradation (ODD) domain of HIF-1 $\alpha$ fused to CreERT2 recombinase, allows us to demonstrate hypoxic cells in the performing brain under normoxia and motor-cognitive challenge, and spatially map them by light-sheet microscopy, all in comparison to inspiratory hypoxia as strong positive control. We report that a complex motor-cognitive challenge causes hypoxia across essentially all brain areas, with hypoxic neurons particularly abundant in the hippocampus. These data suggest an intriguing model of neuroplasticity, in which a specific task-associated neuronal activity triggers mild hypoxia as a local neuron-specific as well as a brain-wide response, comprising indirectly activated neurons and nonneuronal cells.

These authors contributed equally: Umer Javed Butt, Agnes A. Steixner-Kumar, Constanze Depp

Supplementary information The online version of this article (https:// doi.org/10.1038/s41380-020-00988-w) contains supplementary material, which is available to authorized users.

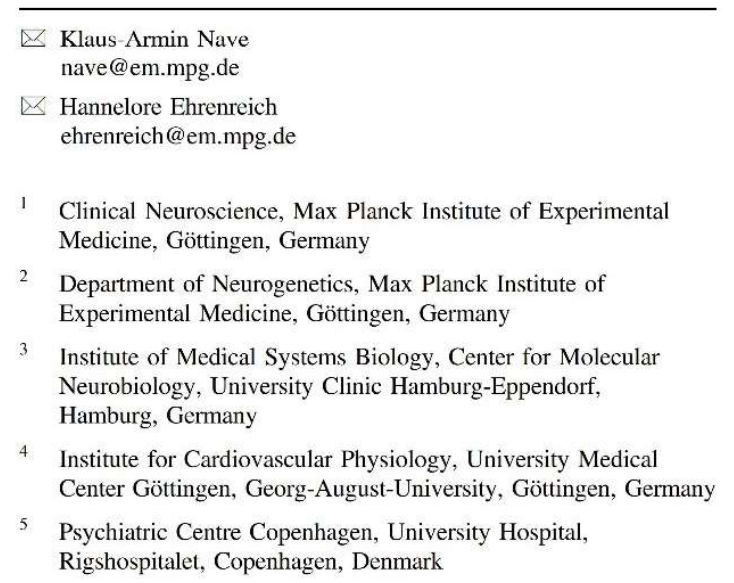

Published online: 09 February 2021

\section{Introduction}

Hypoxia is the term for reduced oxygen levels in cells or tissues, relative to their 'normal' content. In former times interpreted as principally pathological, for instance upon cardiac arrest, hypoxia is increasingly recognized as physiological driving force of early neurodevelopment including angiogenesis, hematopoiesis, and tissue regeneration. Known cellular environments experiencing hypoxia include developing embryos, stem cell niches, the renal papilla, inflammatory tissue, or the inner mass of tumours [1-10]. A specific transcriptional programme, induced by hypoxia, allows cells to adapt to lower oxygen levels and/or to limited metabolic support [11]. The transcription is partly independent of $[10,12]$ and partly controlled by hypoxia-inducible factors (HIF), binding to hypoxia-responsive elements to modulate expression of a myriad of genes, some of which are potent growth factors like vascular endothelial growth factor (VEGF) or erythropoietin (EPO) [10, 13-20].

On one hand, the tight association of neuronal activity with oxygen availability is the basis of functional magnetic 
resonance imaging (fMRI), which works by detecting the level of oxygen in blood throughout the brain. Changes in oxygenation and hemodynamics generate a fast surrogate signal of brain activity based on structural and functional neurovascular coupling [21-23].

On the other hand, extensive physical activity as well as cognitive challenge lead to widespread brain activation, and are ultimately associated with improved global brain function including mood and emotional well-being in health and disease [24, 25]. Neurologists and psychiatrists encourage their patients to improve functions by practicing, following the old rule 'use-it-or-lose-it'. For example, hippocampal volume increases following exercise in both healthy and schizophrenic subjects, and this plastic response correlates with improvement in test scores for short-term memory [26]. Despite these well-established observations, the underlying mechanisms have remained widely obscure.

In several clinical trials targeting different neuropsychiatric diseases, we showed over the last 2 decades that high-dose recombinant human EPO consistently improved cognition and reduced grey matter loss (e.g. [27-30]). Subsequently focusing on preclinical EPO studies for deeper mechanistic insight, we discovered that challenging cognitive tasks apparently induce transient neuronal hypoxia which triggers neuronal EPO expression. Endogenous EPO in turn enhances cognition via augmenting dendritic spine formation and increasing numbers of pyramidal neurons [31, 32]. In this context, we coined the term 'brain EPO circle', and reported an increase in hypoxia-labelled neurons after complex running wheel (CRW) exposure together with an amplified expression of EPO in pyramidal CA1 neurons [32].

Taking all this information together, we designed the present study, hypothesizing that for physiological postnatal and adult adaptation processes in the brain, hypoxia may be a crucial mediator of major general relevance. We show here that complex motor-cognitive activity leads to 'functional hypoxia' as a local, neuronal network-specific, as well as a brain-wide response, encompassing indirectly activated neurons and - to a lesser degree-non-neuronal cells. This activity-induced hypoxia regulates adaptive gene expression and fosters neuroplasticity.

\section{Materials and methods}

Important note: All experiments, including cell counting, were performed by investigators unaware of group assignment and treatments ('fully blinded').

\section{Generation of CAG-CreERT2-ODD transgenic mice}

The p-CAG vector, kindly provided by Hesham A. Sadek [33], was slightly modified (Fig. 1a). In brief, the vector contains the oxygen-dependent-degradation domain (ODD) of HIF-1 $\alpha$, fused with a tamoxifen-inducible cre-recombinase, driven by a ubiquitous CAG promoter. Under normoxic conditions, the ODD is hydroxylated by prolylhydroxylases, which tag HIF-1 $\alpha$ upon binding of Von Hippel-Lindau (VHL) protein for proteasomal degradation. Hypoxic conditions lead to inactivation of these enzymes and thus stabilization of HIF- $1 \alpha$, allowing it to accumulate in the nucleus and activate transcription of its target genes. Analogously, under hypoxic conditions the ODD-crerecombinase fusion construct is stabilized and-in the presence of tamoxifen - translocates to the nucleus to trigger tdTomato expression, which leads to permanent labelling of cells that were transiently exposed to hypoxia [33]. The vector was cloned and bacterial backbone (ampicillin sequence) removed by Dral and SpeI (New England Biolabs, MA, USA) restriction enzymes. The linearized vector for pro-nuclear microinjection was purified by QIAquick Gel Extraction kit (Qiagen, Venlo, Netherlands) and introduced into fertilized eggs for generation of CAG-CreERT2ODD transgenic mice. Transgenic founder mice and the next generations were viable and breeding normally.

\section{Mouse genotyping}

Founder mice from the litters were screened by PCR amplification of genomic DNA for the transgene using following primer pair: forward $5^{\prime}$-GCTGAAGACACAG AAGCAAA- $3^{\prime}$ and reverse $5^{\prime}$-GTGGGTAGGAGATG GAGATG- $3^{\prime}$. Mice carrying the transgene were maintained on C57BL6/N (Charles River, MA, USA) background. F1 litters were bred to Rosa26R-tdTomato reporter mice [34]. For analysis of tdTomato transgene, primer1 5'-TCAATGGGCGGGGGTCGTT-3', primer2 $5^{\prime}$-CTCTGCTGCCTCCTGGCTTCT- $3^{\prime}$ and primer $35^{\prime}$-C GAGGCGGATCACAAGCAATA- $3^{\prime}$ were used. CAGCreERT2-ODD::R26R-tdTomato F2 litters were selected for histological analysis.

\section{Experimental procedures}

All experiments were approved by and conducted in accordance with the regulations of the local Animal Care and Use Committee (Niedersächsisches Landesamt für Verbraucherschutz und Lebensmittelsicherheit, LAVES). CAG-CreERT2-ODD::R26R-tdTomato mice were used at the age of 4 and 24 weeks in all experiments. Mice were single housed in standard plastic cages, starting 2-3 days before the respective experiments, and maintained in temperature-controlled environment $\left(21 \pm 2{ }^{\circ} \mathrm{C}\right)$ on $12 \mathrm{~h}$ light-dark cycle with food and water available ad libitum. Mice of each gender were randomly allocated to experimental and control groups. 
a CAG-creERT2-ODD-PCAG

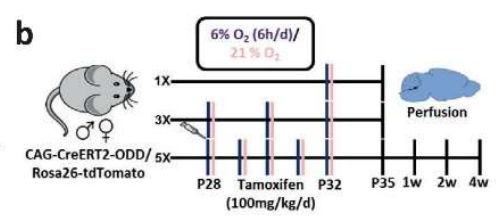

b

Characteristics of tamoxifen-induced ODD labelling of hypoxic neurons in hippocampus

C Hypoxia/tamoxifen dose response
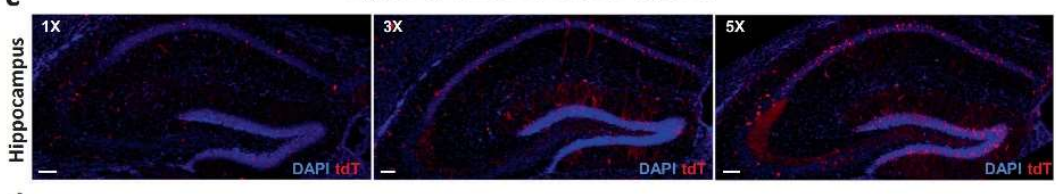

d
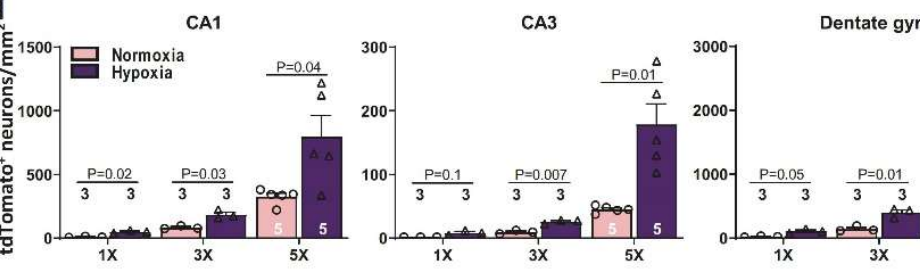

entate gyrus

Oxygen dose response
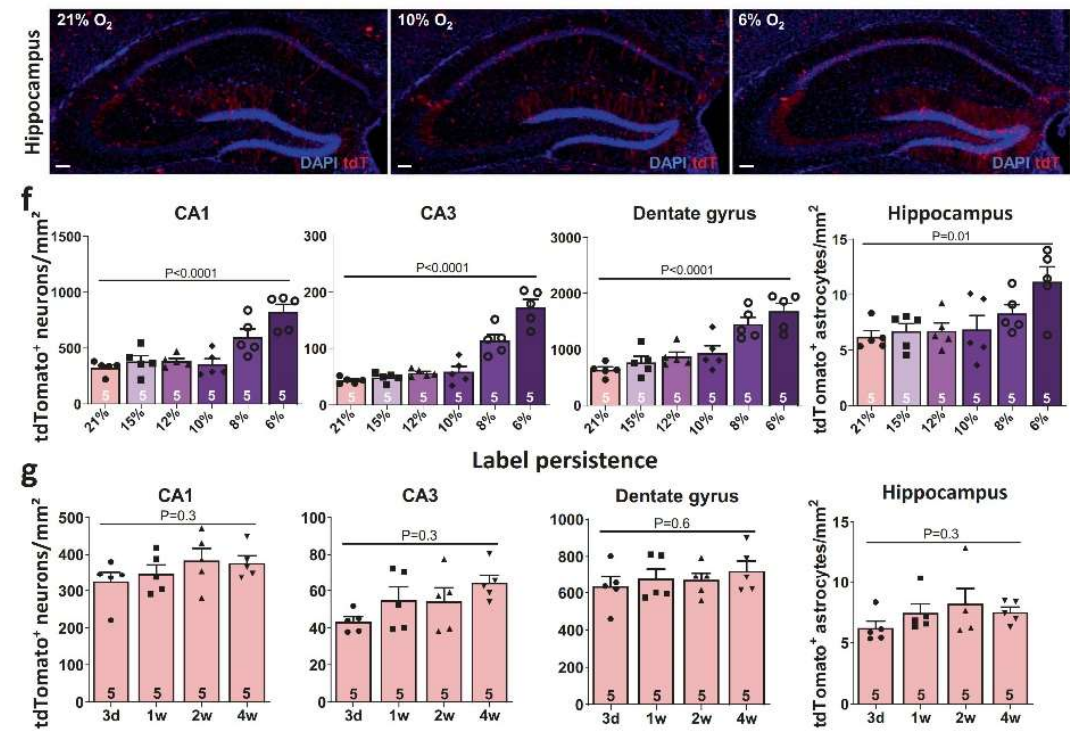

Label persistence
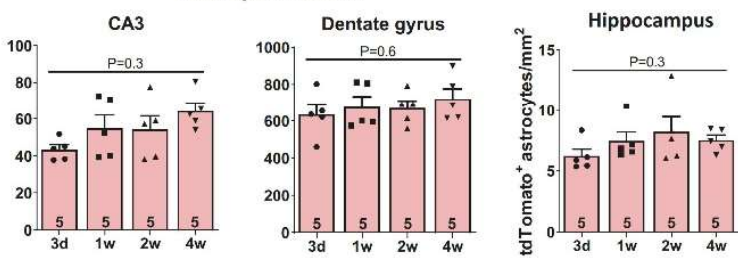

Fig. 1 Generation and characterization of CAG-CreERT2-ODD: R26R-tdTomato mice as model to study hypoxic cells in the brain. a CAG-CreERT2-ODD and CAG-Rosa26R-tdTomato constructs.

b Experimental outline for quantification of hypoxic cells in hippocampal regions $\mathrm{CA} 1, \mathrm{CA} 3$, and dentate gyrus under variable conditions, as displayed in (c-g). After the last day of treatment (P32), mice were sacrificed, perfused and brains harvested for histological analysis at the time points indicated. $\mathbf{c}$ Illustrative images of hippocampus from $1 \times, 3 \times$ and $5 \times$ hypoxia/tamoxifen-treated mice, showing tdTomato + (red) hypoxic cells; DAPI (blue) as nuclear stain. d Quantitative results (based on counting of $\mathrm{tdT}+\mathrm{NeuN}+$ neurons) of the hypoxia/tamoxifen titration scheme, combining $1 \times, 3 \times$ or $5 \times$ placement of mice right after

tamoxifen injection ( $100 \mathrm{mg} / \mathrm{kg} / \mathrm{d}$ i.p.) for $6 \mathrm{~h}$ into a cage with hypoxia exposure $\left(6 \% \mathrm{O}_{2}\right)$ versus a cage with continued normoxia $\left(21 \% \mathrm{O}_{2}\right)$. e Illustrative images ( $5 \times$ tamoxifen), showing tdTomato + (red) hypoxic cells and DAPI (blue) as nuclear stain and $\mathbf{f}$ quantification of the dose-response of hypoxic neurons (based on tdT $+\mathrm{NeuN}+$ cells) and astrocytes (based on tdT $+\mathrm{S} 100 \beta+$ cells) upon decreasing inspiratory $\mathrm{O}_{2}$ concentration $\left(5 \times\right.$ tamoxifen; $21 \% \mathrm{O}_{2}, 15 \% \mathrm{O}_{2}, 12 \% \mathrm{O}_{2}, 10 \% \mathrm{O}_{2}, 8 \% \mathrm{O}_{2}$ and $6 \% \mathrm{O}_{2}$ ). $\mathrm{g}$ Tracking of tamoxifen-induced tdTomato + hypoxic cells over the course of 4 weeks after treatment cessation under normoxic conditions ( $5 \times$ tamoxifen) reveals essentially label persistence; 2 -tailed Welch's $t$-test and 1-way ANOVA were used for statistical analyses; error bars indicate SEM; scale bar represents $100 \mu \mathrm{m}$. 


\section{Tamoxifen injections}

Tamoxifen stock solution was prepared by mixing $300 \mathrm{mg}$ tamoxifen (Sigma, Darmstadt, Germany) in $30 \mathrm{ml}$ of corn oil (Sigma) and stored at $4{ }^{\circ} \mathrm{C} ; 100 \mathrm{mg} / \mathrm{kg}$ tamoxifen was injected intraperitoneally (i.p.) after preheating and sonication for $20 \mathrm{~min}$. To control for non-induced expression of the transgene ('leakiness'), corn oil was injected.

\section{Hypoxia exposure}

A hypoxia chamber $(60 \mathrm{~cm} \times 38 \mathrm{~cm} \times 20 \mathrm{~cm})$ was designed in cooperation with Coy Laboratory Products (Michigan, USA). The hypoxia chamber is equipped with an oxygen sensor, oxygen controller and a ceiling fan for constant air circulation. In hypoxia experiments (Fig. 1b), animals were given a single dose of tamoxifen $(100 \mathrm{mg} / \mathrm{kg})$ before being placed in the hypoxia chamber for $6 \mathrm{~h}$ at $6 \% \mathrm{O}_{2}$. This procedure was repeated at identical time $(10.30-16.30 \mathrm{~h})$ for 5 consecutive days if not stated otherwise. $\mathrm{O}_{2}$ was dropped from 21 to $6 \%$ over the course of $30 \mathrm{~min}$, kept at $6 \%$ for $6 \mathrm{~h}$ and brought back up to $21 \%$ upon cessation of the experiment. Mice included in the normoxia group and oil-only control mice were handled identically but stayed at $21 \% \mathrm{O}_{2}$. For histological studies mice were perfused 3 days after the 5-day experiment (day 8) and brains dissected for further analysis. For single-cell RNAseq analysis, mice were sacrificed immediately after the last hypoxia/normoxia control session on day 5 without perfusion.

\section{Running wheel experiment}

CRW (TSE Systems, Bad Homburg, Germany) are characterized by randomized missing bars as previously described [35, 36] and running activity is computercontrolled via Phenomaster software (TSE Systems, Germany). Running-naïve mice, aged 4 or 24 weeks, were allowed to freely use CRW for 5 whole days. Tamoxifen injections were given daily during the light cycle, along with refill of fresh food and water. Non-running mice were excluded from the experiment (5/24 all together). Control mice were housed without CRW in standard cages. After day 5, CRW mice were returned to standard cages for 3 days before perfusion (day 8).

\section{Tissue preparation and histology}

Mice were anesthetized with Avertin (Tribromoethanol, Sigma-Aldrich, St Louis, MN, USA, $0.276 \mathrm{mg} \mathrm{g}^{-1}$ ) and perfused with $0.9 \%$ saline and pre-chilled $4 \%$ paraformaldehyde (PFA; Sigma, Missouri, United States), followed by brain isolation. All tissues were post-fixed in $4 \%$ formaldehyde at $4{ }^{\circ} \mathrm{C}$ for $24 \mathrm{~h}$ and later cryoprotected in $30 \%$ sucrose prepared in phosphate buffer saline (PBS) for $48 \mathrm{~h}$ at $4{ }^{\circ} \mathrm{C}$. Brains were further covered in cryoprotectant (O.C.T. ${ }^{\text {TM }}$ Tissue-Tek, Sakura, Netherlands) and kept until use at $-80{ }^{\circ} \mathrm{C}$. Whole mouse brains were cut into $30 \mu \mathrm{m}$ thick coronal floating sections using a cryostat (Leica, Wetzlar, Germany) and stored in a cryoprotective solution (25\% ethylene glycol and $25 \%$ glycerol in PBS) at $4{ }^{\circ} \mathrm{C}$ until further use.

\section{Immunofluorescence staining}

For immunofluorescence analysis, 5 sections $(30 \mu \mathrm{m})$ from each mouse were washed and blocked for $1 \mathrm{~h}$ in $5 \%$ horse serum diluted in PBS with $0.5 \%$ Triton $\mathrm{X}-100$ at room temperature (RT). Primary antibodies were diluted in $3 \%$ horse serum PBS/0.3\% Triton X-100 and sections incubated for $48 \mathrm{~h}$ at $4{ }^{\circ} \mathrm{C}$, followed by washing and incubation with respective secondary antibody for $2 \mathrm{~h}$ at RT. For nuclear counterstaining, 4',6-diamidino-2-phenylindole (DAPI, Sigma, Missouri, United States) was used. The sections were then mounted on SuperFrostPlus Slides (Thermo Fisher, Germany) with Aqua-Polymount (Polysciences, Inc USA). For direct visualization of tdTomato, sections were only stained with DAPI and investigated by confocal microscope Leica TCS SP5-II. Primary antibodies used were anti-NeuN (MAB377, 1:1000, Mouse Millipore, Darmstadt, Germany), anti-IBA1 (234006, 1:1000, chicken; SYSY, Göttingen, Germany) and anti-Olig2 (AB9610, 1:1000, rabbit, Millipore), anti-alanyl aminopeptidase membrane (CD13)-AF647 (564352, rat, 1:200, 564352; BD Biosciences, San Jose, CA, USA), Lectin (DL-1174, 1:500, DyLight 488 Lycopersicon Esculentum (Tomato), antiS100ß (287004/1-4, guinea pig, 1:500, SYSY). Secondary antibodies used were Alexa 488 anti-guinea pig (123588, 1:500, Invitrogen), Alexa 647 anti-chicken (121361, 1:500, Jackson ImmunoResearch, Cambridgeshire, UK), Alexa 647 anti-mouse (1757130), Alexa 647 anti-rabbit (1693297) and Alexa 647 anti-rat (A-21247, 1:500; Thermo Fisher).

\section{Confocal imaging and quantification}

For quantification of NeuN+tdTomato + co-labelled cells, serial coronal sections from the dorsal part of hippocampus were taken (coordinates from Bregma: -1.34 to $-2.54 \mathrm{~mm}$ posterior). Stained sections $(30 \mu \mathrm{m})$ were imaged using a Leica confocal microscope TCS SP5 System equipped with a $20 \times$ objective $(\mathrm{NA}=0.70)$. Neurons $(\mathrm{NeuN}+)$ from $\mathrm{CA} 1$, $\mathrm{CA} 3$ and DG were counted on both sides of hippocampus by Fiji software (https://imagej.net/Fiji). NeuN+tdTomato+ cells were considered as neurons having experienced hypoxia. Astrocytes (S100 $\beta+$ tdTomato + ) and endothelial 
cells (Lectin+tdTomato + ) were counted bilaterally in whole hippocampus ( 5 sections per animal). For oligodendrocyte quantification, Olig $2+$ tdTomato + cells were counted in serial coronal sections of the rostral corpus callosum (both sides). Illustrative images were analyzed and processed with Imaris 9.1.0 (www.bitplane.com).

\section{Light-sheet microscopy (LSM)}

\section{Whole mount tissue staining and clearing}

To visualize tdTomato + cells in the entire brain we performed LSM in combination with whole mount immune labelling and tissue clearing. Animals were deeply anesthetized and transcardially perfused using PBS and 4\%PFA/ PBS, respectively. Brains were removed, post-fixed in $4 \%$ PFA overnight and stored in PBS until further use. Brain hemispheres were processed for immune labelling and tissue clearing following a slightly modified iDISCO protocol [37]. Samples were dehydrated with a methanol/PBS series $(50 \%, 80 \%, 100 \%$, and $2 \times 100 \%, 1 \mathrm{~h}, \mathrm{RT})$ followed by overnight bleaching and permeabilization in a mixture of $5 \% \mathrm{H}_{2} \mathrm{O}_{2} / 20 \%$ dimethyl sulfoxide (DMSO) in methanol at $4{ }^{\circ} \mathrm{C}$. Samples were retrieved and washed further with methanol at $4{ }^{\circ} \mathrm{C}$ for $30 \mathrm{~min}$ and $-20^{\circ} \mathrm{C}$ for $3 \mathrm{~h}$ prior to incubation in 20\% DMSO in methanol at RT for $2 \mathrm{~h}$. Samples were then rehydrated using a descending methanol/PBS series $(80 \%, 50 \%$, PBS, $1 \mathrm{~h}$ each, RT) and further washed with in PBS $/ 0.2 \%$ Triton X-100 for $2 \mathrm{~h}$. The samples were then incubated overnight in $0.2 \%$ Triton $\mathrm{X}-100$, $20 \%$ DMSO, and $0.3 \mathrm{M}$ glycine in PBS at $37^{\circ} \mathrm{C}$ and blocked using PBS containing 6\% goat serum, $10 \%$ DMSO and $0.2 \%$ Triton $\mathrm{X}-100$ for 2 days at $37^{\circ} \mathrm{C}$. Samples were retrieved, washed twice in PBS containing $0.2 \%$ Tween(r) 20 and $10 \mu \mathrm{g} / \mathrm{ml}$ heparin (PTwH) at RT for $1 \mathrm{~h}$ and incubated with primary antibody solution (anti-RFP; Rockland \# $600-401-379 ; 1: 250$ in $\mathrm{PTwH} / 5 \% \mathrm{DMSO} / 3 \%$ goat serum) for 14 days at $37^{\circ} \mathrm{C}$. After several washes during the day and an additional overnight wash in PTwH samples were incubated with secondary antibody solution (goat anti-rabbit Alexa555; Thermo Fisher Scientific \# A-21428; 1:500 in $\mathrm{PTwH} / 3 \%$ goat serum) for 7 days at $37^{\circ} \mathrm{C}$. Prior to clearing, the samples were again washed in PTwH (several solution changes during the day) followed by an additional overnight wash. Tissue was dehydrated using an ascending series of Methanol/PBS $(20 \%, 40 \%, 60 \%, 80 \%, 2 \times 100 \% 1 \mathrm{~h}, \mathrm{RT})$ followed by overnight incubation in a mixture of $33 \%$ dichloromethan (DCM) and 66\% methanol at RT. Samples were further delipidated by incubation in 100\% DCM for $40 \mathrm{~min}$ and transferred to pure ethyl cinnamate (Eci; SigmaAldrich \#112372) as clearing agent. Tissues became transparent after $30 \mathrm{~min}$ in Eci and were stored at RT until imaging.

\section{LSM and 3D analysis/visualization}

LSM was performed using a LaVision Ultramicroscope II equipped with a $2 \times$ objective, corrected dipping cap and zoom body. Samples were mounted onto the sample holder with the medial surface of the brain hemisphere facing down in order to acquire sagittal images. The holder was placed into the imaging chamber filled with Eci. Images were acquired in mosaic acquisition mode with the following specifications: $5 \mu \mathrm{m}$ sheet thickness; $20 \%$ sheet width; $2 \times$ zoom; $4 \times 5$ tiling; $4 \mu \mathrm{m}$ z-step size; dual site sheet illumination; $50 \mathrm{~ms}$ camera exposure time; $1000 \mathrm{px} \times$ $1600 \mathrm{px}$ field of view. Red fluorescence was recorded using $561 \mathrm{~nm}$ laser excitation (5-10\%) and 585/40 emission filters. Images were loaded into Vision4D 3.0 (Arivis) and stitched using the tile sorter setup. Hippocampus and cortex regions of interests (ROIs) where manually annotated according to the sagittal Allen mouse brain atlas [38]. For this, hippocampus and cortex ROIs were traced manually in a few planes in 2D from which the 3D ROI was extrapolated automatically. Cortex and hippocampus annotations were then cropped with a medial cut-off of $\sim 0.4 \mathrm{~mm}$ and a lateral cut-off of $\sim 4.4 \mathrm{~mm}$ (corresponding to the lateral end of the hippocampal formation). Cortex ROIs spanned the dorsal parts of the cortex as defined by anatomical landmarks. Next, tdTomato + cells per ROI were identified using the blob finder algorithm in Vision4D. Noise was removed by deleting objects with voxel sizes $<10$ from the object table. Objects were then critically reviewed, and any additional noise was manually removed from the dataset. The number of tdTomato+ cells per ROI was extracted from the object table and plotted using GraphPad (Prism). For visualization purposes, representative single planes of the 3D datasets displayed in inverted grayscale were extracted. To clearly visualize the distribution of tdTomato + cells in the 3D hippocampus, the datasets were cropped along the respective hippocampus ROI, the grayscale 3D renderings were inverted, and a high resolution snapshot of the 3D ROIs was taken. Blobfinder identified objects were further displayed as simplified spheres using the centroid display mode of objects in Vision4D.

\section{Single-cell RNA sequencing}

\section{Isolation and preparation of whole hippocampal cells}

CAG-CreERT2-ODD::R26R-tdTomato mice, 4 weeks old, received tamoxifen $(100 \mathrm{mg} / \mathrm{kg} / \mathrm{d})$ daily for 5 consecutive days before being exposed to either inspiratory hypoxia $\left(6 \% \mathrm{O}_{2}\right)$ or normoxia $\left(21 \% \mathrm{O}_{2}\right)$ for $6 \mathrm{~h}$ each. Mice were handled identically and sacrificed on day 5 immediately after the last hypoxia/normoxia session. Hippocampi were dissected in 
Earle's Balanced Salt Solution (EBSS1) and later digested with a working solution of Papain/DNaseI prepared in EBSS1, according to the manufacturer's instructions (Worthington Biochemical Corp). All steps were carried out on ice except the Papain/DNaseI dissociation. The samples were then incubated at $37^{\circ} \mathrm{C}$ for $40 \mathrm{~min}$ with constant shaking in water bath and switched to $5 \% \mathrm{CO}_{2}$ every $10 \mathrm{~min}$. Papain/DNaseI was removed and samples were further diluted in $5 \mathrm{ml}$ EBSS2. Dissociated tissues were manually triturated (avoid air bubbles), followed by centrifugation at $900 \mathrm{rpm}$ for $10 \mathrm{~min}$ at $4{ }^{\circ} \mathrm{C}$. Supernatant was discarded and the cell pellet gently resuspended in $200 \mu \mathrm{l}$ of EBSS2 until a smooth and creamy suspension was obtained. Dissociated cells were washed frequently with DMEM/F12 (Sigma) without phenol-red containing 3\% foetal bovine serum (FBS; Life Technologies) and centrifuged at $900 \mathrm{rpm}$ for $10 \mathrm{~min}$ at $4{ }^{\circ} \mathrm{C}$. Cell pellet was resuspended in $1 \mathrm{ml}$ of DMEM/F12 and filtered through a $70 \mu \mathrm{m}$ strainer cap (Corning) smoothly to harvest a clear cell suspension. Cell viability and yield was calculated by mixing an equal volume of acridine orange and propidium iodide and checked at the cell counter (Nexcelom Bioscience, MA, USA). Cell suspension $(1 \mathrm{ml})$ was diluted with $3 \mathrm{ml}$ PBS containing $0.04 \%$ BSA and centrifuged. Supernatant was removed and cell pellet resuspended in $400 \mu \mathrm{lBS}$ containing $0.04 \%$ BSA. Cells were fixed by adding $1.6 \mathrm{ml}$ methanol dropwise and kept at $-20^{\circ} \mathrm{C}$ for $30 \mathrm{~min}$ and stored at $-80^{\circ} \mathrm{C}$ for further use.

\section{Cell rehydration, single-cell library preparation and RNA sequencing (scRNA-seq)}

The Methanol fixed cell suspensions were stored at $-80^{\circ} \mathrm{C}$ for less than 7 days before processing. For each sample, $\sim 1.5$ million cells were taken for rehydration and downstream scRNA-seq experiment. Cells were first placed on ice and equilibrated to $4{ }^{\circ} \mathrm{C}$. After equilibration, cells were washed 2 times by $1 \mathrm{ml}$ pre-chilled rehydration buffer $(1.0 \%$ BSA, $0.5 \mathrm{U} / \mu \mathrm{l}$ RNAse inhibitor in $1 \times$ Dulbecco's Phosphate-Buffered Saline, DPBS). Centrifugation was done at $3000 \mathrm{rcf}$ for $10 \mathrm{~min}$ at $4{ }^{\circ} \mathrm{C}$. Finally, cells were resuspended in pre-chilled $1 \times$ DPBS with $0.04 \%$ BSA and $0.5 \mathrm{U} / \mu \mathrm{l}$ RNase inhibitor to achieve a concentration of 1000 cells/ $\mu l$. Rehydrated cells were immediately used for the scRNA-seq using the $10 \times$ genomics chromium single-cell gene expression platform. Around 35,000 cells from each sample were loaded on 1 well of $10 \times$ Single Cell A chip, where each single cell was lysed and its transcriptome combined with a single barcoded gel bead in an oil droplet. Barcode cDNA libraries were then prepared using Chromium Single Cell $3^{\prime}$ Library and Gel-beads kit v2 according to the manufacturer's instructions. Library quality was checked using Agilent High Sensitivity DNA chip on Agilent 2100 Bioanalyzer. High quality libraries were sequenced on Illumina HiSeq 4000 sequencer with an average depth of 200,000 reads per cell.

\section{Alignment and initial processing of sequencing data}

CellRanger v2.2.0 software was used to align sequence reads to the customized mouse $\mathrm{mm} 10$ reference genome to which the ODD and tdTomato sequences were added. A filtered gene expression matrix was generated by CellRanger and was afterwards loaded into Seurat for further analysis.

\section{Single-cell sequencing data analysis}

Additional quality control as well as sample integration, cell clustering and marker gene identification were done with $\mathrm{R}$ (v3.4.1) [39] using Seurat packages v2.3.0 (quality control, normalization) [40] and v3.0.0 (scaling, integration, clustering, differential expression) [41]. Genes that were detected in $\geq 3$ cells and cells in which the number of detected genes ranged between 500 and 6000 (hypoxia samples) or 7500 (normoxia samples) respectively were retained for further analysis. In addition, cells that contained $>20 \%$ mitochondrial genes were removed, resulting in a total number of 28,114 cells, expressing altogether 20,976 genes. After integration and clustering, another 2264 cells that were likely to be doublets upon examination of marker genes and/or predicted to be doublets by DoubletFinder [42] were excluded. This led to the final dataset containing 25,850 cells (normoxia: $n=12,341$, hypoxia: $n=13,509$ ) and 20,976 genes. This dataset was used for further analysis and graphical display. Gene expression levels were normalized via natural-log normalization of gene transcripts divided by total transcripts per cell and scaled by 10,000 . Data integration of the 4 samples was done using the 20 first dimensions of CCA (canonical correlation analysis) and by calling the functions FindIntegrationAnchors() and IntegrateData(). After scaling of integrated data and dimension reduction through principal component analysis, uniform manifold approximation and projection (UMAP) dimension reduction was performed on the first 30 principal components. Subsequently, nearest neighbors were identified using the FindNeighbors() function and clusters were determined with FindClusters (resolution $=0.2$ ). This initially revealed 21 cell clusters. After removal of doublet (sub)clusters (determined by co-expression of 2 main celltype markers) and merging of clusters that were highly similar with regard to main marker expression, 16 different cell types were identified. Identification of cell types was based on differentially expressed genes (function FindAllMarkers $(\operatorname{logfc}$.threshold $=0.5)$ ) in each cluster and expression of known marker genes [43]. The FindMarkers() function was employed to determine differential expression 
of Vegfa and $H k 2$ between hypoxia and normoxia. A positive $\log \mathrm{FC}$ indicates higher expression under hypoxia as compared to normoxia. Percentages of ODD, tdTomato or double-positive cells as well as $H k 2+$ cells were determined by calculating the proportion of cells with non-zero expression in the respective genes. For all feature plots showing normalized expression levels, a minimal and maximal cut-off was set at 0.5 and 2 , respectively.

\section{Extraction of mRNA and real-time quantitative reverse transcription polymerase chain reaction (qPCR)}

Juvenile female WT (C57BL/6N) P32 mice were sacrificed after $6 \mathrm{~h}$ of normoxia, hypoxia or CRW (during their active phase, i.e., lights-off) and hippocampi were dissected and directly frozen on dry ice. Total RNA was extracted from hippocampal tissue by using miRNeasy Mini Kit (Qiagen, Hilden, Germany). The cDNA was prepared using SuperScript III Reverse Transcriptase (Thermo Fisher Scientific Life Technologies GmbH, Darmstadt, Germany), and $1 \mu \mathrm{g}$ of RNA along with oligo (dT) and Random Hexamer Primer in a total volume of $20 \mu \mathrm{l}$. The qPCR was performed as described in detail earlier [31, 44]. For qPCR, $4 \mu \mathrm{l}$ of 1:10 diluted cDNA were used as template with $5 \mu$ of Power SYBR Green PCR Master Mix (Thermo Fisher Scientific Life Technologies) and $1 \mathrm{pmol}$ of primers (in $1 \mu \mathrm{l} \mathrm{H}_{2} \mathrm{O}$ ).

For $\mathrm{N}$-myc downstream-regulated gene 1 protein (Ndrgl), nitric oxide synthase 1 (Nosl), Ankyrin repeat domain-containing protein 37 (Ankrd37), HIG1 domain family member 1A (Higdla), VEGF A (Vegfa), erythropoietin (Epo), enolase 2 (Eno2), beta-actin (Act $\beta$ ) and hypoxanthine guanine phosphoribosyl transferase (Hprtl) the following primers were used:

Ndrgl forward primer:

5'-CGAAGACCACCCTGCTCAAG-3'

Ndrgl reverse primer:

5'-ATGCTGGCAGAAGGCATGTAT-3'

Nos 1 forward primer: $5^{\prime}$-CATCAGGCACCCCAAGTT- ${ }^{\prime}$

Nos $I$ reverse primer:

5'-CAGCAGCATGTTGGACACA- $3^{\prime}$

Ankrd37 forward primer:

5'-AAACAGGTGCTGACCTCAACC- ${ }^{\prime}$

Ankrd37 reverse primer:

5'-CAGTCCAGGCTTCCAACCTTT-3'

Higdla forward primer:

5'-ACGATGAAGGTCAGGGGTCT-3'

Higdla reverse primer:

5'-AGGCAACAATCGCTGCAAAG- ${ }^{\prime}$

Vegfa forward primer:

5'-AGCACAGCAGATGTGAATGC- ${ }^{\prime}$

Vegfa reverse primer:

5'-TTGACCCTTTCCCTTTCCTC-3'
Epo forward primer:

5'-AAGGTCCCAGACTGAGTGAAAATATTAC- ${ }^{\prime}$

Epo reverse primer: $5^{\prime}$-GGACAGGCCTTGCCAAACT $-3^{\prime}$

Eno2 forward primer:

5'-TGGAGTTTGGGGAGTGCTGGATG- ${ }^{\prime}$

Eno2 reverse primer:

5'-AGGGCTGGGGAGAGGGTTAGAGG-3'

$\beta$-actin forward primer:

$5^{\prime}$-CTTCCTCCCTGGAGAAGAGC-3'

$\beta$-actin reverse primer:

$5^{\prime}$-ATGCCACAGGATTCCATACC- ${ }^{\prime}$

Hprtl forward primer:

5'-GCTTGCTGGTGAAAAGGACCTCTCGAAG-3'

Hprtl reverse primer:

5'-CCCTGAAGTACTCATTATAGTCAAGGGCAT- ${ }^{\prime}$

The qPCR reactions ( 3 technical replicates) were run on LightCycler ${ }^{\circ} 480$ System (Roche, Mannheim, Germany). Fold difference in mRNA expression was calculated by the $\Delta \Delta \mathrm{Ct}$ method and normalized to Hprtl and $\beta$-actin.

\section{Statistical analyses}

Data obtained for all quantifications were analyzed by GraphPad Prism 7 (GraphPad Software, Inc. San Diego, CA, USA). Statistical significance between multiple groups was calculated by 1-way or 2-way analysis of variance (ANOVA). Welch's $t$-test was performed to compare two groups. Jonckheere-Terpstra trend test was applied to identify stepwise increases in data. General and cell-typespecific differences in numbers of $H k 2+$ cells were assessed via Chi-square tests (unadjusted $P$ values reported). Differential expression (scRNA-seq) was evaluated by Wilcoxon rank sum test (Bonferroni-corrected $P$ values reported, if not declared otherwise). A $P$ value $<0.05$ was considered statistically significant. Bar graphs show means and error bars represent standard error of mean (SEM). Sample sizes were selected based on previous work in order to allow sufficient statistical power to detect differences with a minimum number of animals (RRR principle). Datasets were routinely screened for statistical outliers using Grubb's test and outliers were excluded if indicated $(P<0.05)$

For intercorrelation analyses and calculation of the gene composite score, following the reported standard operating procedure [44, 45], gene expression was Z-standardized. Intercorrelation between qPCR-genes was plotted using Rpackage 'corrplot'. Cronbach's alpha was used as measure of internal consistency and $\alpha>0.6$ considered acceptable. For calculation of the composite score, Z-standardized single gene expression values were averaged across all 7 genes. 


\section{Results and discussion}

\section{Transgenic approach to permanent labelling of hypoxic brain cells}

To test our hypothesis that neuronal activity leads to 'functional hypoxia', we employed a transgenic mouse line (CAG-CreERT2-ODD::R26R-tdTomato) that allows permanent reporter labelling of cells that undergo hypoxia by expression of a fusion protein. This fusion protein is comprised of the ODD domain of HIF-1 $\alpha$ and a tamoxifeninducible CreERT2, driven by a ubiquitous CAG promoter (slightly modified from Kimura et al. [33]) (Fig. 1a). Using this transgenic approach, we first defined our 'gold standard' of a prominent positive control by comparing mice exposed to intermittent inspiratory hypoxia $\left(6 \% \mathrm{O}_{2}\right.$ for $6 \mathrm{~h}$ daily, applied over 5 days) with mice under inspiratory normoxia $\left(21 \% \mathrm{O}_{2}\right)$ that were handled identically, including the five tamoxifen injections as indicated (Fig. 1b).

\section{Characterization of the model: hypoxia/tamoxifen and oxygen dose-response curves (inspiratory hypoxia) as well as persistence of labelling}

Based on this 'gold standard' (tamoxifen injection, followed by $6 \% \mathrm{O}_{2}$ for $6 \mathrm{~h}$ daily, applied over 5 days), we determined the numbers of tdTomato+ cells (mainly neurons-see below) in cornu ammonis hippocampi, namely in CA1, which is a well-defined, highly cognition-relevant, and at the same time established hypoxia-vulnerable brain region (Sommer's sector) [46-49], and in CA3, as well as in the dentate gyrus (DG) [50,51]. As deducible from the scheme in Fig. 1b, in pilot experiments, hypoxia days/tamoxifen injections $(100 \mathrm{mg} / \mathrm{kg} /$ day $)$ had first been experimentally 'titrated' by applying $1 \times, 3 \times$ or $5 \times$ hypoxia $\left(6 \% \mathrm{O}_{2}\right.$ for $6 \mathrm{~h}$ daily, each preceded by a tamoxifen injection) and compared to respective normoxia days/tamoxifen injections. This led to a clear dose-response of the number of tdTomato + cells to hypoxia/tamoxifen and our final decision to stay with $5 \times$ hypoxia/tamoxifen as our 'gold standard' in the following experiments (Fig. 1c, d).

We also explored in initial tests the influence of the degree of inspiratory hypoxia on the number of tdTomato + neurons and obtained an 'oxygen dose-response curve' that started to rise appreciably only at $8 \% \mathrm{O}_{2}$, and made us select the $6 \% \mathrm{O}_{2}$ for our subsequent experiments (Fig. 1e, f).

Using pimonidazole as an immediate hypoxia marker for further confirming hypoxia mapping [4] allowed us at least to identify few scattered tdTomato + cells that showed double-labelling, very similar to what was presented in a recent paper on heart [33]. Unfortunately, the temporal dynamics of tdTomato labelling (visible earliest $\sim 6-8 \mathrm{~h}$ after hypoxia exposure) and pimonidazole which can be maximally applied for $90 \mathrm{~min}$ before the staining becomes increasingly unspecific, do not permit more extensive colabelling (Supplementary Fig. 1).

Finally, we analyzed in preparatory work tdTomato labelling at various time points ( 3 days to 4 weeks) after completed exposure to the standard schedule of $5 x$ tamoxifen injections under inspiratory normoxia $\left(21 \% \mathrm{O}_{2}\right)$, which showed essentially persistence of labelling over up to 4 weeks (Fig. 1g). In all these experiments, characterizing our transgenic model, 4-week-old male and female mice were equally distributed across experimental groups.

\section{Light-sheet microscopy discloses a distinct increase in labelled cells from baseline cage activity to inspiratory hypoxia}

We next applied light-sheet microscopy (LSM) to obtain a 3-dimensional (3D) overview of tdTomato+ (ODD) labelling in whole brain (Fig. 2a-c) [37, 52]. Because of the slightly stronger response of female mice to the hypoxia stimulus (see below), we opted for females at the age of 4-5 weeks. Comparative 3D visualization of hypoxic cells revealed a striking, regionally distinct distribution pattern across the entire brain. Similar to our histological quantification (Fig. 1c, d), the amount of labelled cells all over the brain increased markedly from mice with just baseline cage activity to animals exposed to our defined 'gold standard' of inspiratory hypoxia $\left(6 \% \mathrm{O}_{2}\right.$ for $6 \mathrm{~h}$ daily, applied over 5 days) (Fig. 2a-c). To control for non-induced expression of the transgene ('leakiness' of tdTomato labelling), corn oil ('no tamoxifen') was injected, which led to an only small percentage of labelled cells (quantified for normoxia in LSM, Fig. 2c; same depiction upon hypoxia, as screened by histology). We note that the olfactory bulb, the lead sensory organ of mice, showed an extraordinarily strong tdTomato+ labelling upon tamoxifen across conditions, offering in this species an 'internal control of functional hypoxia' (Fig. 2a-c and Supplementary Video I).

\section{Hypoxic cells in cortex and hippocampus are predominantly neurons}

We next explored the immunohistochemical nature of hypoxia signal-positive cells in cortex and hippocampus (representative illustrating images upon our defined 'gold standard' of inspiratory hypoxia given in Fig. 3a-e). Comparing normoxia (just baseline cage activity) with exogenous/inspiratory hypoxia (and later with endogenous/ activity-related hypoxia; see below) revealed essentially quantitative, no noticeable qualitative differences between conditions. In other words, the cell types labelled under normoxia and exogenous hypoxia (as well as later under CRW performance as endogenous/activity-related hypoxia; 


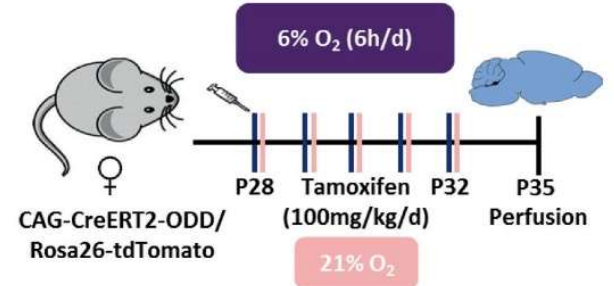

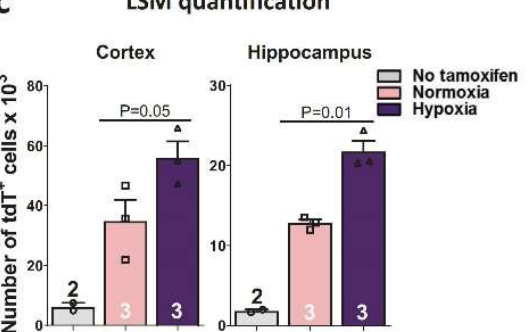

b
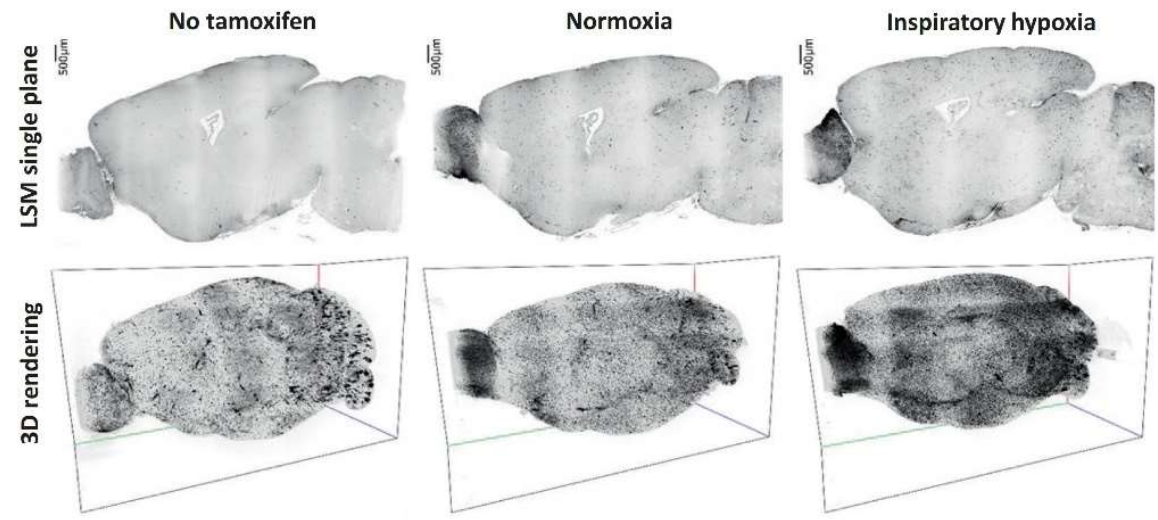

Fig. 2 Light-sheet microscopy (LSM) allows spatial mapping of hypoxic cells in whole brain using CAG-CreERT2-ODD::R26R reporter mice. a Experimental outline. b Light-sheet microscopy (LSM) 2D sagittal planes and maximal intensity projection 3D renderings of tdTomato + cells in the entire brain hemispheres; images

shown in inverted grey scale; scale in 3D images given by bounding box: $x$ axis (green) $10 \mathrm{~mm}, y$ axis (red) $6.2 \mathrm{~mm}, z$ axis (blue) $4.2 \mathrm{~mm}$. c Quantification of tdTomato + cells in 3D hippocampus and cortex; 2-tailed Welch's $t$-test; error bars indicate SEM.

see below for quantifications), and their overall distribution were widely corresponding. Remarkably, the overwhelming fraction of tdTomato+ cells were neurons, followed by endothelial cells, whereas labelled astrocytes (mainly protoplasmic), oligodendrocytes and pericytes were much scarcer and displayed some regional differences. Rare, labelled oligodendrocytes, for instance, appeared mainly in white matter areas, whereas no labelled oligodendrocytes were found in the hippocampus. Surprisingly, microglia were never tdTomato+ labelled.

\section{Single-cell transcriptomes show comparable expression of ODD and tdTomato in hippocampal cell lineages under normoxia or hypoxia}

To exclude a merely technical problem as underlying cause of these surprising cellular differences in labelling, we determined whether the ODD-CreERT2 transgene, harbouring a ubiquitously active (CAG) promotor, is indeed equally expressed in all cell types. For this, we employed single-cell transcriptome analysis of whole hippocampus as a most sophisticated and comprehensive approach.
This approach confirmed comparable expression of both transgenes (ODD, tdTomato) in all hippocampal cell types under normoxia as well as upon our defined 'gold standard' of inspiratory hypoxia (Fig. 4a-c, Supplementary Fig. 2). As an example, we verified Vegfa as a prototypical hypoxiainducible gene, upregulated in many cell types under hypoxia (for bulk comparison normoxia-hypoxia: $P=8.96 \mathrm{e}$ $-22)$ and particulary highly expressed in OPC and astrocytes (avg_logFC $>0.25, \quad p_{\text {unadj }}<0.05 ; \quad$ Supplementary Fig. 3a, b).

\section{Hexokinase2 expression is inversely related to the amount of tdTomato + cells in each cell type}

Searching for a first explanation of the apparent 'hypoxia tolerance' of microglia (never tdTomato+ labelled in any gender or treatment condition), we made an unexpected discovery. The expression of hexokinase 2 ( $H k 2$; catalyzing the first step of glycolysis) under hypoxia was found inversely related to the amount of tdTomato + cells in each cell type (Fig. 4d). Cell types with a high percentage of $H k 2+$ cells under hypoxia showed a lower number of 


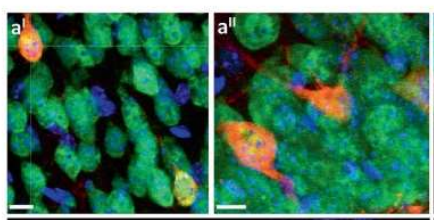

a
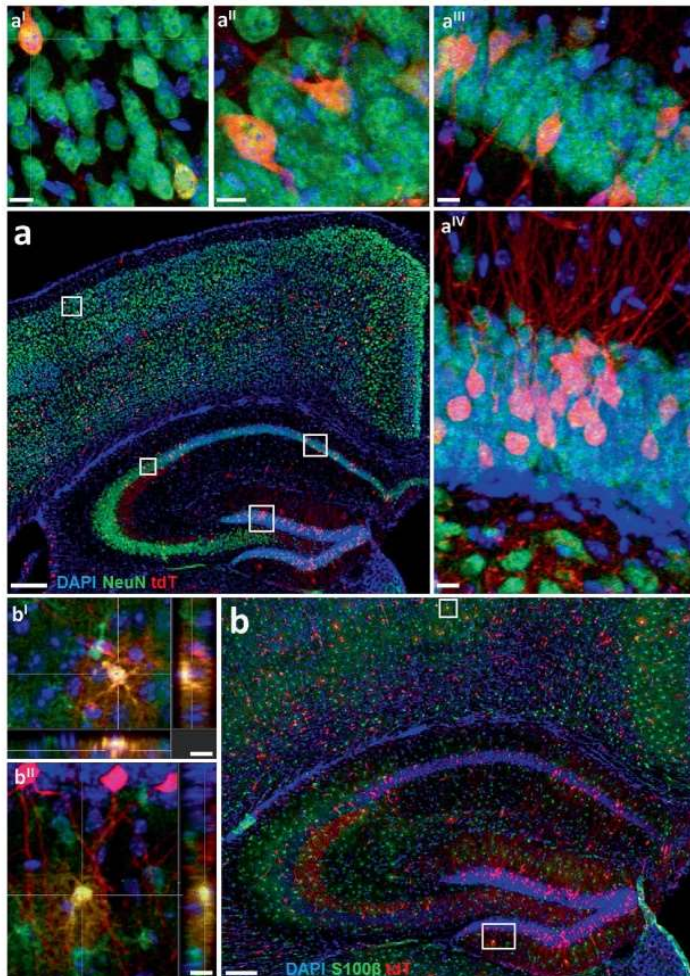

Fig. 3 Confocal images illustrate the predominance of neurons among hypoxic cells in hippocampus and cortex of CAGCreERT2-ODD::R26R-tdTomato mice. Shown are representative images obtained upon our defined 'gold standard' of inspiratory hypoxia. Note that normoxia and CRW (endogenous/activity-related 'functional hypoxia') revealed quantitative, no noticeable qualitative differences of labelled cell types. a High density of tdTomato $+\left(\mathrm{tdT}^{+}\right.$, red) neurons shown by co-labelling with the neuronal marker $\mathrm{NeuN}$ (green). Magnified confocal images of $\mathrm{tdT}^{+} \mathrm{NeuN}^{+}$neurons depict cortex $\left(\mathrm{a}^{\mathrm{I}}\right)$, hippocampal CA2 $\left(\mathrm{a}^{\mathrm{II}}\right), \mathrm{CA} 1\left(\mathrm{a}^{\mathrm{III}}\right)$, and dentate gyrus, DG $\left(\mathrm{a}^{\mathrm{IV}}\right)$. b S100ß-stained astrocytes (green) show less frequent

tdTomato + cells. Accordingly, microglia showed the most prominent expression compared to all other cell types under normoxic and hypoxic conditions (normoxia, $P=6.02 \mathrm{e}-7$, $\log \mathrm{FC}=0.07 ;$ hypoxia, $P=6.85 \mathrm{e}-15, \quad \log \mathrm{FC}=0.29$ ). Oligodendrocytes also exhibited high $H k 2$ expression under hypoxia in the hippocampus. In line with this, no labelled oligodendrocytes appeared within the hippocampus, but predominantly (yet rarely) in white matter tracts (see also below). Hk2 is a glycolytic enzyme, well-recognized from tumour biology to promote cell survival by hypoxia resistance $[53,54]$. Under inspiratory hypoxia, increased numbers of $H k 2+$ cells were identified among almost all cell types $(P<0.05$ compared to the respective cell-type-specific normoxia numbers) (Fig. 4c, d). Even though highly interesting, Hk2 may not be the only explanation for the

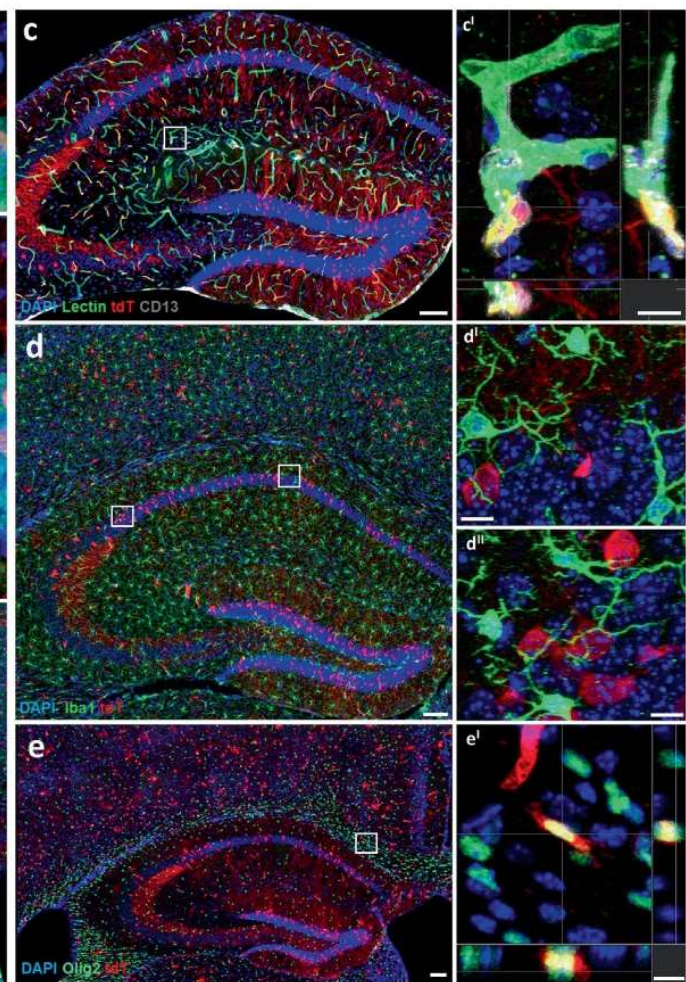

co-immunostaining with tdTomato. $\left(\mathrm{b}^{\mathrm{I}}\right)$ and $\left(\mathrm{b}^{\mathrm{II}}\right)$ display protoplasmic astrocytes from cortex and DG with orthogonal views. $\mathrm{c}$ Overview of hippocampal endothelial cells (lectin+, green) and pericytes (CD13+, grey), some co-immunostained with tdTomato; $\left(\mathrm{c}^{\mathrm{T}}\right)$ zoom-in image of co-labelled cells. $\mathbf{d}$ Images of microglia staining (lbal, green). $\left(\mathrm{d}^{\mathrm{l}}\right)$ and $\left(d^{I I}\right)$ represent magnified images from CA1 and CA2, documenting lack of co-localization of Ibal with tdTomato. e Olig2 staining (green) reveals sparse double labelling with tdTomato as illustrated in $\left(\mathrm{e}^{\mathrm{l}}\right)$ depicting cells from the corpus callosum. Scale bars represent $200 \mu \mathrm{m}$ in overview and $10 \mu \mathrm{m}$ in zoom-in images.

observed differences between cell types. Notably, however, we did not observe on mRNA level any indicative expression patterns under normoxia or hypoxia of prolyl-hydroxylases, VHL gene or factor inhibiting hypoxia-inducible factor 1 that could potentially explain differences in tdTomato labelling due to different activity of the ODD degradation pathway in different cell types.

Nonetheless, the absence of tdTomato labelling in microglia is a highly intriguing finding. In some agreement with studies on brain tumours [55], we see in our scRNAseq data that microglia clearly respond with an upregulation of hypoxia-related transcripts, yet it remains unclear why no ODD stabilization takes place. This could be partially explained by the high $\mathrm{Hk} 2$ expression, protecting microglia indirectly from a strong decrease in oxygen levels which 

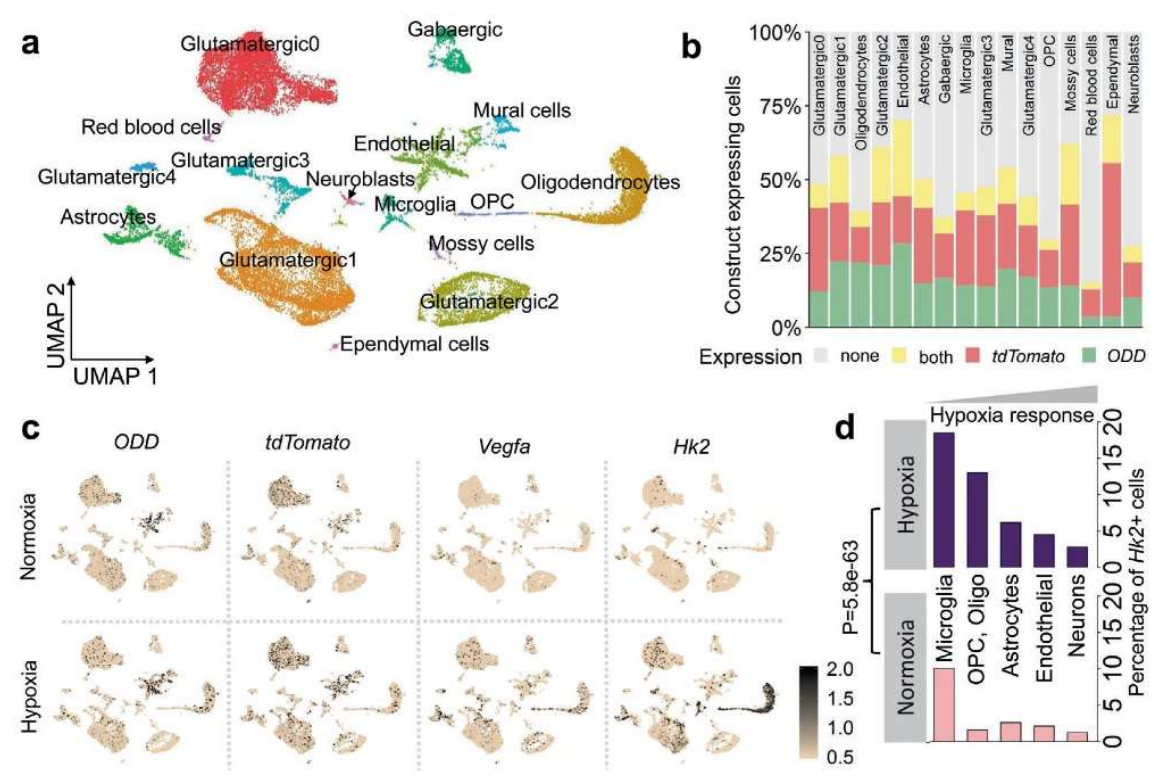

Fig. 4 Single-cell transcriptome analysis shows overall construct expression and indicates a role for hexokinase 2 in the differentia cellular hypoxia response. a Unbiased clustering of hippocampal cells from mice under normoxia $(n=2)$ or hypoxia $(n=2)$, represented in UMAP space, reveals 16 distinct cell populations across conditions. OPC: oligodendrocyte precursor cells. b ODD and tdTomato mRNAs are present in all cell types at comparable levels; percentage of cells shown where either ODD, tdTomato, both transcripts together, or none were detected (normalized expression $>0$ ). c Expression plots of ODD, tdTomato, and of 2 hypoxia-regulated genes, Vegfa and $H k 2$. Constructs ODD and tdTomato are expressed at

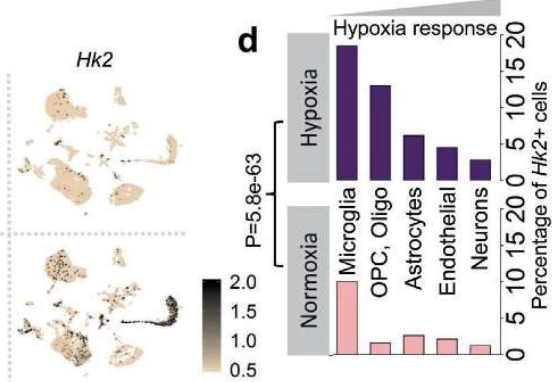

similar levels in all cell types. $H k 2+$ cells were ordered by expression level (highly expressing cells plotted on top by setting FeaturePlot $($ order $=\mathrm{T})$ ) to prevent masking of relatively rare $H k 2+$ cells. d Percentage of detectable $H k 2$ expressing cells increases to a varying degree in all cell types (borderline in endothelial cells under hypoxia). Microglia reveal the highest number of $H k 2$ expressing cells under normoxia and a considerable increase under hypoxia; a strong increase under hypoxia is also observed in OPC and oligodendrocytes. For easier visualization, the cluster of ependymal cells, which was located far away from all other clusters, was shifted upwards on the UMAP2 axis (a, c). Chi-square test presented.

would be required for ODD stabilization. Alternatively, a different level of oxygen-dependence of microglia ('setpoint'), a divergent activity of the HIF- $1 \alpha$ degradation pathway or cell-type-related molecular features preventing ODD-stabilization could be relevant in this context. Possibilities range e.g. from different abundance of prolylhydroxylases or VHL protein, deviating availability of iron, ascorbate, tricarboxylic acid cycle intermediates, or levels of radical oxygen species (some only shown in vitro to be relevant) [56]. Moreover, less tight coupling of microglial and vascular oxygen levels (possibly related to distance from capillaries) may affect ODD-stabilization in microglia. All these speculations will have to be experimentally addressed in the future.

\section{Number of tdTomato + neurons upon motor- cognitive challenge suggests 'functional hypoxia'}

Based on the characterization of our model and its validation by intermittent exogenous/inspiratory hypoxia, we next approached our hypothesis that neuronal activity leads to 'functional hypoxia'. For this purpose, we selected a multifaceted, demanding learning task for mice, i.e., running on complex wheels $[35,36]$. CRW performance requires hippocampal involvement, but also activation of the motor circuits, somatosensory or visual cortex, and recruitment of the prefrontal-corticostriatal path [57-59]. Randomized missing bars, requiring continuous steplength adaptation and bilateral coordination characterize CRW. The CRW task is entirely dependent on voluntary mouse activity and constitutes a marked cognitive challenge that stimulates complicated pattern recognition, intricate motor learning, and respective coordination. We thus compared mice, running on complex wheels, employed to induce our postulated, activity-related, 'functional endogenous hypoxia' ('CRW'; under inspiratory normoxia; $21 \% \mathrm{O}_{2}$ ), with mice upon spontaneous cage activity (unchallenged 'normoxia' controls; $21 \% \mathrm{O}_{2}$ ) and mice exposed to our 'gold standard' of a prominent positive control ('hypoxia'; $6 \% \mathrm{O}_{2}$ for $6 \mathrm{~h}$ daily) - all conditions applied over 5 days. Animals of all groups were handled identically, including tamoxifen injections as indicated 
U. J. Butt et al.

(Fig. 5a-c). In this series of experiments, we included also gender and age comparison. Hence, we determined tdTomato+ (ODD labelled) neurons upon normoxia (basal activity), cognitive challenge (CRW) or inspiratory hypoxia $\left(6 \% \mathrm{O}_{2}\right)$, in males and females separately, as well as in 2 different age groups ( 4 and 24 weeks, Fig. 5c), focusing again on $\mathrm{CA} 1, \mathrm{CA} 3$ and $\mathrm{DG}$ as our regions of interest. Altogether, a characteristic stair pattern from normoxia over CRW to hypoxia became obvious (Jonckheere-Terpstra trend test for both genders and time points significant), with female mice showing slightly higher amounts of tdTomato + cells (for CAl: interaction treatment $\times$ gender: 4 weeks: $P_{\text {interaction }}=0.048$, 24 weeks $P_{\text {interaction }}=0.018$; for CA3: $P_{\text {interaction }}=0.08$ and

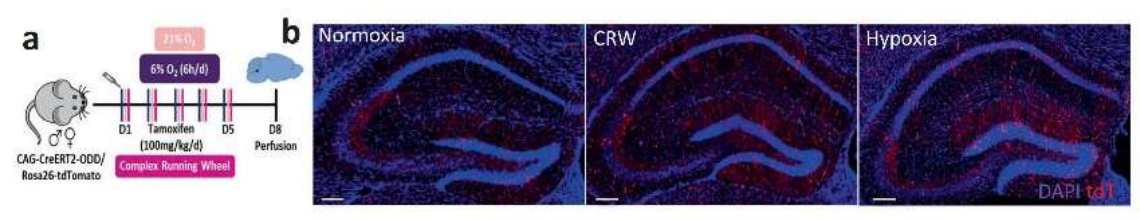

C ODD-tdTomato labelling upon normoxia (basal activity), motor-cognitive challenge (CRW)
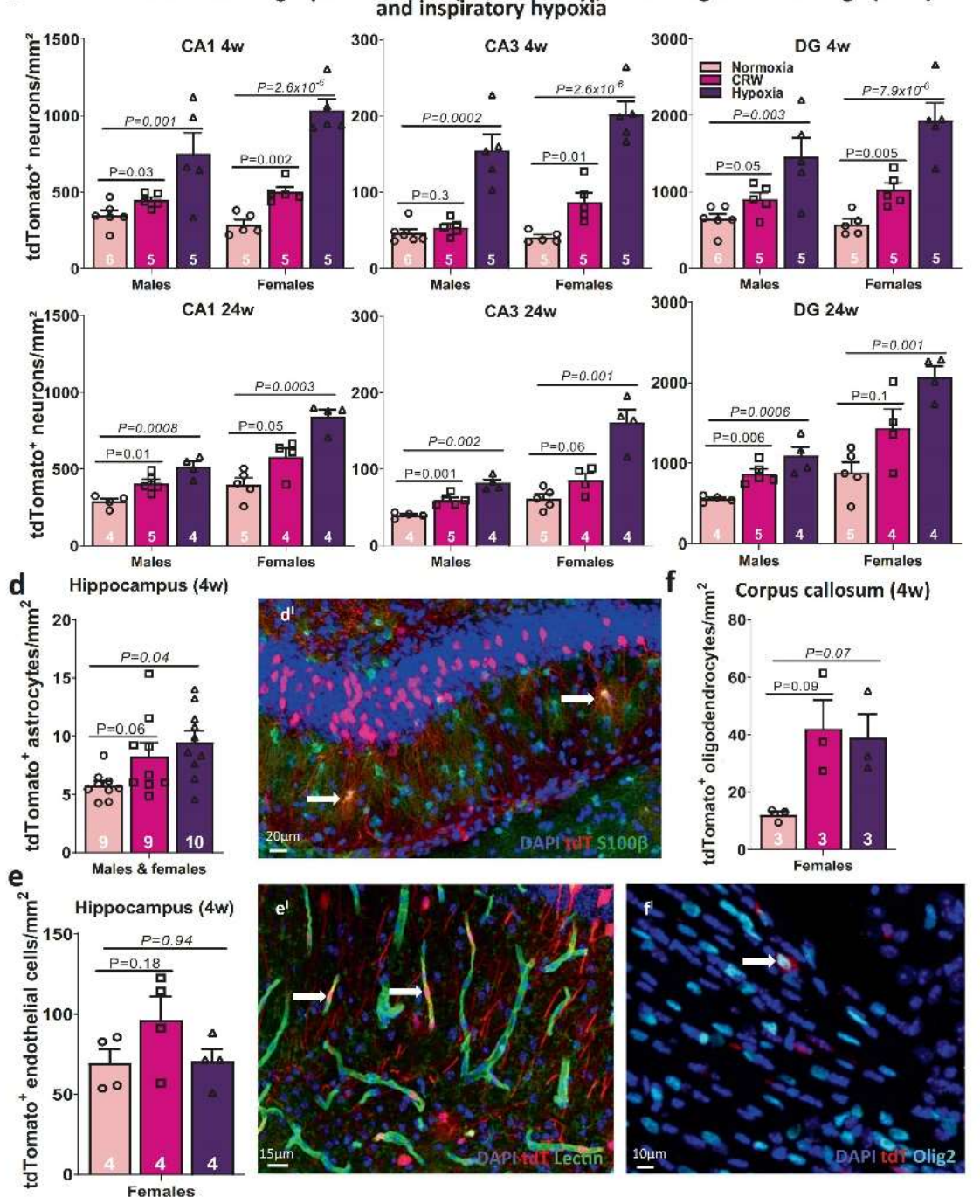

SPRINGER NATURE 
4 Fig. 5 Number of tdTomato + neurons in hippocampus afte motor-cognitive challenge (CRW) versus inspiratory hypoxia support the concept of 'functional hypoxia'. a Experimental outline. b Representative images of the hippocampus upon all 3 conditions. c Comparative quantification of labelled pyramidal neurons $(\operatorname{tdT}+\mathrm{NeuN}+)$ in CA1, CA3 and DG of 4 and 24 week-old CAGCreERT2-ODD::R26R-tdTomato mice of both genders upon spontaneous home cage activity (normoxia), CRW performance and inspiratory hypoxia ('gold standard'). $\left(\mathbf{d}+\mathbf{d}^{\prime}\right)$ The low numbers of hypoxic astrocytes $(\mathrm{tdT}+\mathrm{S} 100 \beta+)$ in whole hippocampus of both genders display a stair pattern similar to neurons with increase upon CRW and inspiratory hypoxia in 4 week-old mice. $\left(\mathbf{e}+\mathbf{e}^{\prime}\right)$ Quantification of hypoxic endothelial cells (tdT + Lectin + ) under normoxia, CRW and hypoxia in whole hippocampus of 4-week-old female mice does not yield significant differences between conditions. $\left(\mathbf{f}+\mathbf{f}^{\prime}\right)$ Quantification of hypoxic oligodendrocytes (tdT+Olig2+) in corpus callosum under normoxia, CRW and hypoxia in 4-week-old female mice shows a strong tendency of increases under both CRW and hypoxia. White arrows in $\mathrm{d}^{\prime}, \mathrm{e}^{\prime}$ and $\mathrm{f}^{\prime}$ illustrate quantified doublepositive cells; two-tailed Welch's test for two-group comparison between normoxia and CRW ( $P$ value non-italicized). JonckheereTerpstra trend test (with 20,000 permutations in $\mathrm{d}-\mathrm{f}$ ) for comparing all three groups ( $P$ value in italics); error bars indicate SEM.

$P_{\text {interaction }}=0.0001 ;$ for DG: $P_{\text {interaction }}=0.1$ and $P_{\text {interaction }}$ $=0.09$, respectively). Interestingly, this is in line with previous reports on gender differences in response to hypoxia [60-62], reflecting e.g. sex steroidal or oestrous cycle influence, perhaps in combination with a genderdiverse ventilatory response or stress perception during single housing. Of note in this context, even cultured male neurons, being more resistant under normoxia, are more vulnerable under strong hypoxia than female neurons and, interestingly, the male vulnerability pattern is acquired in cells from neonatally testosterone-primed females [63]. Age did not seem to play an appreciable role in the 2 groups (4 and 24 weeks) examined.

To provide a representative impression of the magnitude of the hypoxia response, we quantified in CA1 the percentage of hypoxic, i.e., tdTomato+ neurons among all neurons. These comprised in 4 -week-old males $3.70 \pm 0.35 \%$ upon normoxia, $4.85 \pm 0.26 \%$ upon $\mathrm{CRW}$ and $8.02 \pm 1.37 \%$ upon hypoxia. In 4-week-old females, fractions amounted to $3.13 \pm 0.33 \%, 5.44 \pm 0.46 \%$ and $11.2 \pm 0.78 \%$, respectively In 24-week-old males they reached $2.51 \pm 0.16 \%, 4.43 \pm$ $0.35 \%$ and $5.66 \pm 0.41 \%$, and in 24-week-old females $4.34 \pm 0.54 \%, 6.37 \pm 0.75 \%$ and $9.28 \pm 0.63 \%$ (all trend test $P$ values $<0.01)$. Importantly, CRW performance as pronounced motor-cognitive challenge, enhanced number of tdTomato + neurons over normoxia throughout, strengthening our hypothesis that neuronal activity leads to 'functional hypoxia', i.e. activity-induced endogenous hypoxia.

Interestingly, also quantification of tdTomato + astrocytes in whole hippocampus revealed a characteristic stair pattern from normoxia over CRW to hypoxia (Fig. 5d). The percentage of hypoxic astrocytes, i.e. tdTomato + of all astrocytes, encompassed under normoxia $0.90 \pm 0.06 \%$, CRW $1.30 \pm 0.17 \%$ and hypoxia $1.49 \pm 0.14 \%$ (trend test $P=0.004)$. In contrast, no significant differences in the number of hypoxic endothelial cells in whole hippocampus were observed between the three conditions (Fig. 5e), with percentages amounting to $7.98 \pm 0.91 \%$ for normoxia, $9.11 \pm 1.34 \%$ for CRW and $7.77 \pm 0.86 \%$ for hypoxia (trend test $P=0.61)$. Overall, we cannot exclude additional region-specific differences (on top of hippocampus), since we did not systematically quantify other brain regions (except for whole cortex in LSM, see below). To provide an extra overview, coronal brain sections illustrate overall brain tdTomato labelling following normoxia versus CRW (Supplementary Fig. 4a, b).

Since in whole hippocampus, there were no hypoxialabelled tdTomato + oligodendrocytes found (in perfect accordance with the high $H k 2$ expression of these cells, compare Fig. 4d), we had to move to corpus callosum for representative quantifications. There we saw a comparably strong tendency of an increase in hypoxic (tdTomato+) oligodendrocytes upon both, CRW and hypoxia (Fig. 5f). Percentages were as follows: $0.53 \pm 0.11 \%$ for normoxia, $1.26 \pm 0.14 \%$ for CRW and $1.91 \pm 0.06 \%$ for hypoxia (trend test $P=0.002$ ). These cell-type-specific findings-in addition to neurons - are highly interesting but will have to be investigated in more depth in future work, employing e.g. different reporter systems and challenges.

We next planned to demonstrate by cFos labelling that induced neuronal activity leads to 'functional hypoxia', searching for double-labelling by cFos and tdTomato+ of those neurons that have been activated during CRW. However, the ODD-tdTomato labelling occurs much too late, i.e., when cFos expression has already disappeared; thus, doublelabelling cannot be detected due to the different cFos/ODDtdTomato kinetics [32]. For instance, at $4 \mathrm{~h}$ after CRW start, there is plenty of cFos labelling (e.g. cFos + neurons $/ \mathrm{mm}^{2}$ in CA1: non-runner controls, $40 \pm 20.83$, versus CRW, $150 \pm$ 24.36; $P=0.01$ ), however, essentially no ODD-tdTomato labelling visible yet.

\section{Hippocampal mRNA expression of hypoxia- regulated genes after $\mathbf{6 h}$ of CRW supports 'functional hypoxia'}

CRW exposure enhanced the number of tdTomato+ (ODD labelled) neurons over normoxia throughout, supporting that neuronal activity induces 'functional hypoxia'. Interestingly, intensive treadmill exercise has been reported earlier to increase expression of HIF- $1 \alpha$ and its downstream transcript targets [64]. Nevertheless, we next measured hypoxia-regulated gene expression as an additional way of verifying that motor-cognitive challenge causes cellular/ neuronal hypoxia. For this, we extracted mRNA from 

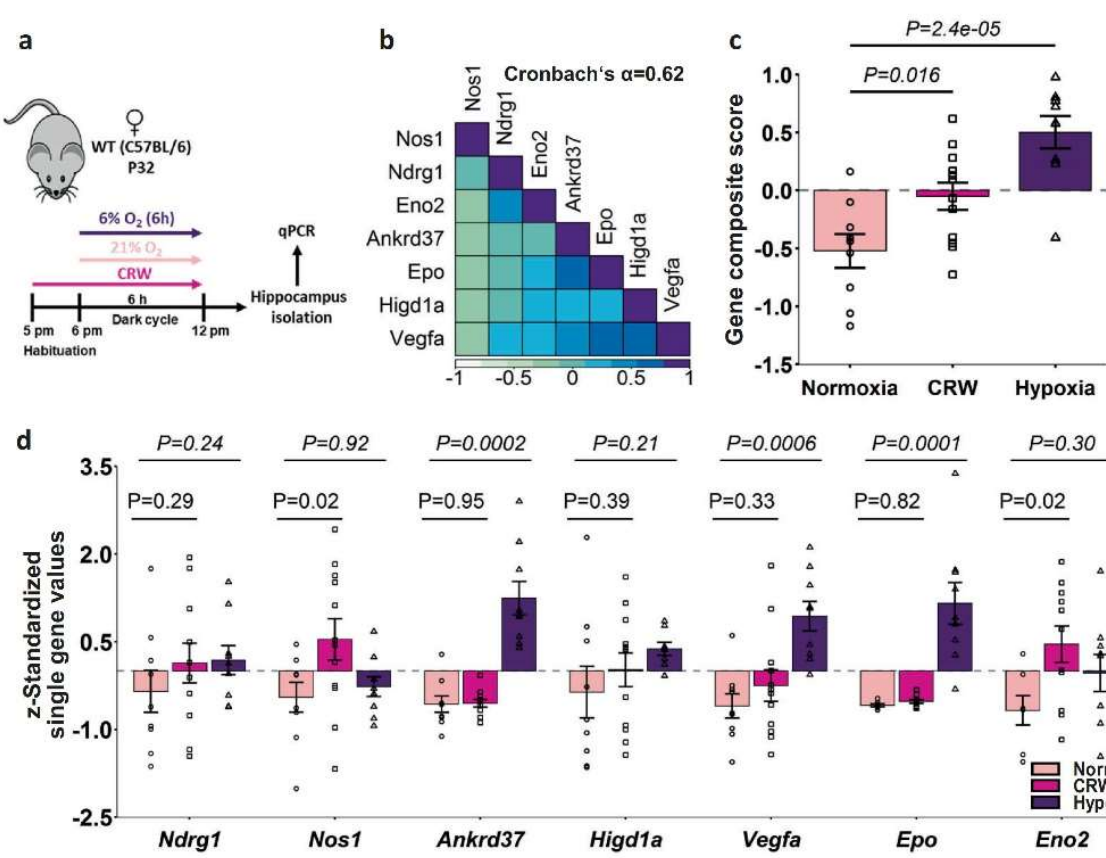

Fig. 6 Selected hypoxia-regulated genes' mRNA expression in hippocampus after $6 \mathrm{~h}$ of motor-cognitive challenge versus inspiratory hypoxia support 'functional hypoxia'. a Experimental outline for hippocampal mRNA expression analyses by qPCR b Intercorrelation pattern and Cronbach's alpha of selected hypoxiaregulated genes. $\mathbf{c}$ Composite gene score shows clear stepwise increase from normoxia over CRW to hypoxia. d Z-transformed qPCR results

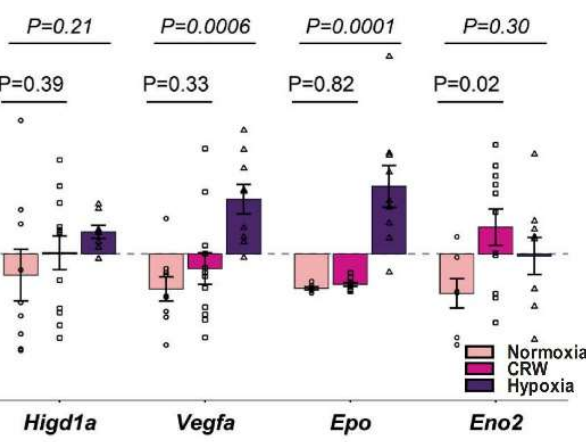

show comparative expression stair patterns (normalized to Hprtl and $\beta$-actin) of all selected single genes, namely $\mathrm{Ndrg} 1$, Nos 1 , Ankrd37, Higdla, Vegfa, Epo, and Eno2; $t$-test with pooled standard deviation for two-group comparison between normoxia and CRW ( $P$ value nonitalicized); Jonckheere-Terpstra trend test $(20,000$ permutations in $(\mathbf{d}))$ for comparing all three groups ( $P$ value in italics); error bars indicate SEM.

hippocampus of mice, exposed for $6 \mathrm{~h}$ in a highly standardized fashion to either normoxia, hypoxia or CRW (Fig. 6a-d). As target genes, we selected Ndrgl, Nosl, Ankrd37, Higdla, Vegfa, Epo and Eno2, all confirmed by our scRNA-seq of whole hippocampus or by respective literature $[32,65]$ to be hypoxia-regulated in neurons and other cell types. We determined mRNA of Epo as a very potent hypoxia-inducible gene, which had escaped scRNAseq analysis due to its very low expression, a known dropout effect of this methodology. For Epo, we know from our previous work using in situ hybridization, that CRW induces its expression in pyramidal neurons, however, peaking at $9 \mathrm{~h} \mathrm{[32].}$

For most of these selected genes, we see a similar picture as with ODD-tdTomato quantifications, with stepwise increase in hippocampal mRNA expression from normoxia over CRW to hypoxia (Fig. 6d). Although gene expression changes under CRW appear to be slight and are often not nominally significant, the consistently obtained pattern (trend test significant or gene expression CRW/hypoxia $>$ normoxia) at a single defined time point $(6 \mathrm{~h})$ is still remarkable, considering variable individual gene

expression dynamics and the fact that we exposed mice to only $6 \mathrm{~h}$ of $\mathrm{CRW}$, a relatively mild stimulus when compared to $6 \% \mathrm{O}_{2}$ for $6 \mathrm{~h}$, as used in our positive control. Nevertheless, since we aimed at proving with our selected transcripts and the obtained data that CRW performance indeed causes cellular/neuronal hypoxia, resembling the inspiratory hypoxia configuration, we performed intercorrelation analyses. Based hereon, we calculated a novel composite score of gene expression, following our previously described standard operating procedure [44, 45]. The internal consistency of the mRNA expression data is reflected by a Cronbach's alpha of $>0.6$, allowing us to calculate the transcript composite scores for normoxia, CRW and hypoxia, respectively, which turned out to be clearly significant (Fig. 6b-d).

\section{LSM mapping of tdTomato + cells upon normoxia versus motor-cognitive challenge indicates 'functional hypoxia' in the behaving brain}

Having substantiated by transcript analyses that motorcognitive challenge causes endogenous hypoxia, we 


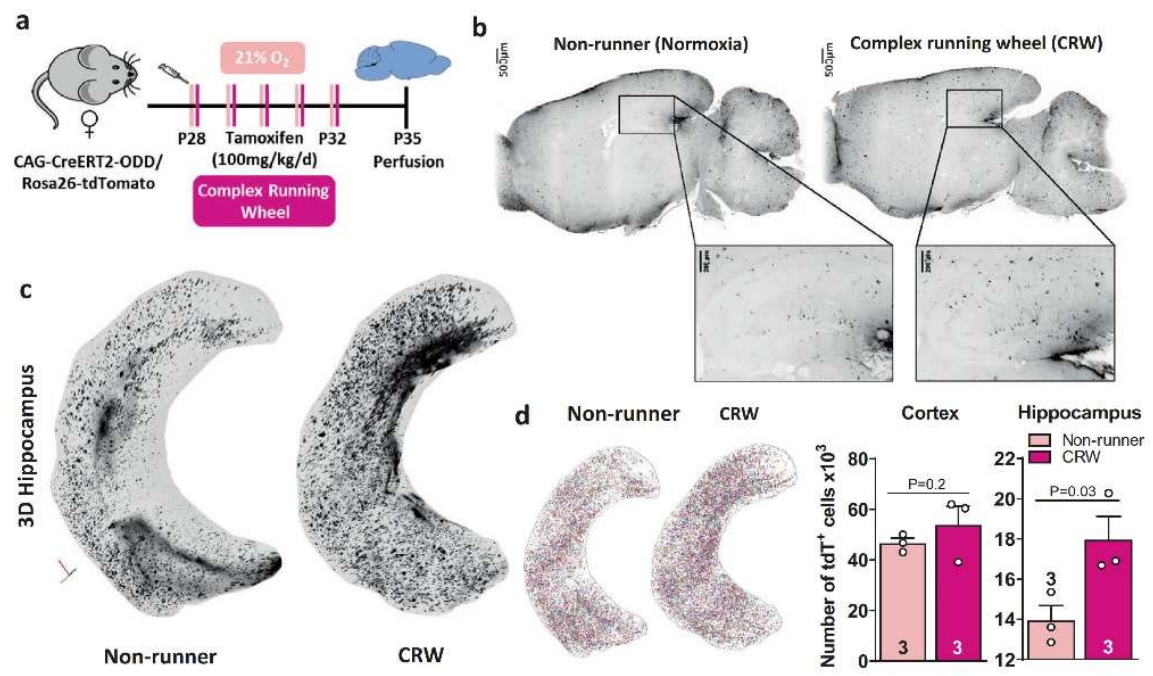

Fig. 7 LSM mapping of tdTomato+ cell distribution upon motorcognitive challenge indicates 'functional hypoxia' in the behaving brain. a Experimental outline. b LSM 2D sagittal planes of tdTomato+ cells; images in inverted grey scale; magnified views of hippocampal area. $\mathbf{c}$ Dorsal view of 3D hippocampi. Hippocampi cropped from LSM hemisphere dataset and visualized as 3D maximal intensity projection; orientation given in left corner. d Quantification of tdTomato + cells in hippocampus and cortex; left images display 3D renderings shown in (d) with tdTomato+ cells represented as random-coloured blobs; 1-tailed Welch's $t$-test, error bars indicate SEM. conducted a second round of LSM analyses. We directly compared female non-runner mice (normoxia) with mice exposed to CRW (Fig. 7a-d). Display in 3D and quantification of tdTomato+ cells in hippocampus revealed a marked increase upon CRW, again strongly supporting our 'functional hypoxia' hypothesis (Fig. 7a-d; Supplementary Video II). Strikingly, however, upon performing a particular task (here: $\mathrm{CRW}$ ) requiring an activation of defined groups of neurons, one might perhaps expect more region-specific activation. The fact that whole hippocampus (and not just cornu ammonis) showed an increase in tdTomato + cells, and that even the cortex displayed a respective tendency, seems less puzzling in light of recent reports that demonstrate a global/brainwide neuronal activation during certain tasks and behaviours in mice [66] and zebrafish [67]. Moreover, even normal wheel running leads to increased brain activation (measured by $\mathrm{cFos}$ expression) in the majority of 25 investigated brain regions [68]. Thus, the focused 'functional hypoxia' in hippocampal regions upon CRW that we see is embedded in a global/brain-wide neuronal activation. Of note, some degree of overreporting by the used reporter system, caused e.g. by tamoxifen-induced cellular stress [69], cannot be entirely ruled out. While overall, this does not invalidate the above conclusions, future studies might want to co-administer vitamin $\mathrm{E}$ with tamoxifen in order to reduce any potential undesired cellular stress.

\section{Working model of activity-induced hypoxia as a driver of neuroplasticity}

The present data suggest a novel working model in which energy-consuming neuronal activity induces hypoxia with its well-known transcriptional programme that-via HIF stabilization-includes metabolic adaptations and the expression of potent growth factors like EPO and VEGF [10, 13-16]. According to this model, activity-induced physiological hypoxia hereby acts as a critical driver of neuroplasticity, engaging e.g. brain-expressed EPO [32]. Notably, recent work even widened our understanding of the hypoxia response, highlighting hundreds of genes expressed under low oxygen, yet most unrelated to HIF $[10,12]$. This suggests that the entire hypoxia response is only in part detectable with our ODD reporter that depends on HIF stabilization. The mechanism is even more complex, as HIF- $1 \alpha$ stabilization can also be stimulated e.g. by the PI3K-Akt-mTOR pathway [70], suggesting that HIF-1 $\alpha$ dependent gene regulation is kept in a cell-type-specific physiological window. Thus, also CreERT2-ODD dependent activation of the reporter gene may be cell typespecifically co-regulated at the cellular level, partly explaining response differences between cell types, as discussed above and exemplified particularly by microglia that appear nearly unresponsive to hypoxia.

Therefore, all data presented in this manuscript have to be understood in this greater context. We are also aware that 
functional hypoxia due to specific neuronal network activity, as induced here by CRW, might with our reporter system not equally be detectable in all theoretically involved brain regions (hippocampus versus motor cortex), since HIF may not be the exclusive mediator of the entire hypoxia response. In fact, we see with our reporter a brainwide 'general' neuronal hypoxia answer to tasks such as complex motor-cognitive learning, on top of stimulated specific areas like the hippocampal cornu ammonis (demonstrated in Video II). This response includes the participation of indirectly activated neurons and of nonneuronal cells in adjusting the brain to specific activityinduced challenges. CRW being a strong motor-cognitive test, we do expect both components (general and specific) to be involved. In addition, 'systemic reactions' ('out of breath'), evoked by physical exercise, may add globally to this particular hypoxia response. Future experiments will further tackle these thought-provoking, still open questions and help disentangle the relative contributions of general versus specific components.

To conclude, our 'functional hypoxia model' integrates not only the specific activity-induced hypoxia, as described here for the hippocampus, but may also help understanding previously unexplained observations. These range from the 'hypoxia vulnerability' of brain areas highly relevant for cognition, as the CA1 region of the hippocampus (Sommer's Sector) [46-49], or the observed HIF-1 $\alpha$ expression upon exercise in activity-involved brain areas [64], to the beneficial effects of HIF stabilizers on cognitive performance [16]. Moreover, our model suggests a general response to specific activity-induced neuronal challenges, such as complex motor-cognitive tasks, which includes indirectly activated neurons and non-neuronal cells. The widespread 'functional hypoxia' arising from increasing energy demands may explain the advantageous effects of physical and cognitive challenges on brain dimensions and global brain function. A picture of hypoxia-induced adaptive neuroplasticity in the postnatal/adult brain is emerging that could also provide the ground for exploring the beneficial therapeutic role of hypoxia in pathological states.

\section{Data availability}

Raw and processed scRNA-seq data are publicly available on GEO via accession code GSE162079.

\section{Code availability}

Analysis scripts for scRNA-seq analysis are available at https://github.com/AgnesSteixner/Butt_et_al_ODD_hypoxia

Acknowledgements This study was supported by the Max Planck Society, the Deutsche Forschungsgemeinschaft (DFG, German
Research Foundation) Research Center for Nanoscale Microscopy and Molecular Physiology of the Brain (CNMPB) as well as by the DFG -TRR 274/1 2020 - 408885537. UJB has received a PhD stipend from National University of Sciences and Technology (NUST), Faculty Development Program Abroad 2014/15 Pakistan. AAS has held a stipend of the IMPRS-GGNB Ph.D. Program Neurosciences (DFG Grant GSC 226), Göttingen. CD holds a Boehringer Ingelheim Fonds Ph.D. Fellowship. TS and SB obtain funding from SFB 1286 Project Z2. KWM received a 5-year Lundbeck Foundation Fellowship (grant no. R215-2015-4121). KAN is supported by Adelson Medical Research Foundation and an ERC Advanced Grant.

Author contributions Concept, design and supervision of the study: HE. Data acquisition/analysis/interpretation: UJB, AAS, CD, TS, LW, IH, SA, MZ, NS, LFG, LM, UB, AR, MH, SG, SB, DMK, KWM, KAN and HE. Drafting manuscript: HE, together with UJB, AAS, CD and KAN. Drafting display items: UJB, AAS, CD, together with HE. All authors read and approved the final version of the manuscript.

Funding Open Access funding enabled and organized by Projekt DEAL.

\section{Compliance with ethical standards}

Conflict of interest The authors declare that they have no conflict of interest.

Publisher's note Springer Nature remains neutral with regard to jurisdictional claims in published maps and institutional affiliations.

Open Access This article is licensed under a Creative Commons Attribution 4.0 International License, which permits use, sharing, adaptation, distribution and reproduction in any medium or format, as long as you give appropriate credit to the original author(s) and the source, provide a link to the Creative Commons license, and indicate if changes were made. The images or other third party material in this article are included in the article's Creative Commons license, unless indicated otherwise in a credit line to the material. If material is not included in the article's Creative Commons license and your intended use is not permitted by statutory regulation or exceeds the permitted use, you will need to obtain permission directly from the copyright holder. To view a copy of this license, visit http://creativecommons. org/licenses/by/4.0/

\section{References}

1. Kaelin WG, Ratcliffe PJ. Oxygen sensing by metazoans: the central role of the HIF hydroxylase pathway. Mol Cell. 2008;30: 393-402.

2. Ratcliffe PJ. Oxygen sensing and hypoxia signalling pathways in animals: the implications of physiology for cancer. J Physiol. 2013;591:2027-42.

3. Semenza GL. Targeting HIF-1 for cancer therapy. Nat Rev Cancer. 2003;3:721-32.

4. Kullmann JA, Trivedi N, Howell D, Laumonnerie C, Nguyen V, Banerjee SS, et al. Oxygen tension and the VHL-Hiflalpha pathway determine onset of neuronal polarization and cerebellar germinal zone exit. Neuron. 2020;106:607-623 e605.

5. Ivashkiv LB. The hypoxia-lactate axis tempers inflammation. Nat Rev Immunol. 2020;20:85-86.

6. Huang X, Trinh T, Aljoufi A, Broxmeyer HE. Hypoxia signaling pathway in stem cell regulation: good and evil. Curr Stem Cell Rep. 2018:4:149-57. 
7. Morikawa T, Takubo K. Hypoxia regulates the hematopoietic stem cell niche. Pflug Arch. 2016;468:13-22.

8. Taylor CT, Colgan SP. Regulation of immunity and inflammation by hypoxia in immunological niches. Nat Rev Immunol. 2017;17:774-85

9. Baik AH, Jain IH. Turning the oxygen dial: balancing the highs and lows. Trends Cell Biol. 2020;30:516-36.

10. Lee P, Chandel NS, Simon MC. Cellular adaptation to hypoxia through hypoxia inducible factors and beyond. Nat Rev Mol Cell Biol. 2020;21:268-83.

11. Yuen TJ, Silbereis JC, Griveau A, Chang SM, Daneman R, Fancy SP, et al. Oligodendrocyte-encoded HIF function couples postnatal myelination and white matter angiogenesis. Cell. 2014;158 383-96.

12. Jain IH, Calvo SE, Markhard AL, Skinner OS, To TL, Ast T, et al. Genetic screen for cell fitness in high or low oxygen highlights mitochondrial and lipid metabolism. Cell. 2020;181:716-727 e711.

13. Kaelin WG. Proline hydroxylation and gene expression. Annu Rev Biochem. 2005;74:115-28.

14. Kenneth NS, Rocha S. Regulation of gene expression by hypoxia. Biochemical J. 2008;414:19-29.

15. Marti HH. Erythropoietin and the hypoxic brain. J Exp Biol. 2004:207:3233-42.

16. Adamcio B, Sperling S, Hagemeyer N, Walkinshaw G, Ehrenreich $\mathrm{H}$. Hypoxia inducible factor stabilization leads to lasting improvement of hippocampal memory in healthy mice. Behavioural Brain Res. 2010;208:80-84.

17. Brines M, Cerami A. Emerging biological roles for erythropoietin in the nervous system. Nat Rev Neurosci. 2005;6:484-94.

18. Suresh S, Rajvanshi PK, Noguchi CT. The many facets of erythropoietin physiologic and metabolic response. Front Physiol. 2020;10:1534.

19. Schuler B, Vogel J, Grenacher B, Jacobs RA, Arras M, Gassmann M. Acute and chronic elevation of erythropoietin in the brain improves exercise performance in mice without inducing erythropoiesis. FASEB J. 2012;26:3884-90.

20. Jelkmann W. Erythropoietin: structure, control of production, and function. Physiological Rev. 1992;72:449-89.

21. Logothetis NK, Pfeuffer J. On the nature of the BOLD fMR contrast mechanism. Magn Reson Imaging. 2004;22:1517-31

22. Hillman EMC. Coupling mechanism and significance of the BOLD signal: a status report. Annu Rev Neurosci. 2014;37: 161-81.

23. Raichle ME. Behind the scenes of functional brain imaging: a historical and physiological perspective. Proc Natl Acad Sci USA 1998;95:765-72.

24. Erickson KI, Hillman $\mathrm{CH}$, Kramer AF. Physical activity, brain and cognition. Curr Opin Behav Sci. 2015;4:27-32.

25. Kramer AF, Erickson KI. Capitalizing on cortical plasticity: influence of physical activity on cognition and brain function. Trends Cogn Sci. 2007;11:342-8.

26. Pajonk FG, Wobrock T, Gruber O, Scherk H, Berner D, Kaizl I, et al. Hippocampal plasticity in response to exercise in schizophrenia. Arch Gen Psychiatry. 2010;67:133-43.

27. Ehrenreich H, Fischer B, Norra C, Schellenberger F, Stender N, Stiefel M, et al. Exploring recombinant human erythropoietin in chronic progressive multiple sclerosis. Brain. 2007;130:2577-88

28. Ehrenreich H, Hinze-Selch D, Stawicki S, Aust C, KnolleVeentjer S, Wilms S, et al. Improvement of cognitive functions in chronic schizophrenic patients by recombinant human erythropoietin. Mol Psychiatry. 2007;12:206-20.

29. Miskowiak KW, Vinberg M, Christensen EM, Bukh JD, Harmer CJ, Ehrenreich H, et al. Recombinant human erythropoietin for treating treatment-resistant depression: a double-blind, randomized, placebo-controlled phase 2 trial. Neuropsychopharmacology. 2014;39:1399-408.

30. Miskowiak KW, Vinberg M, Macoveanu J, Ehrenreich H, Koster $\mathrm{N}$, Inkster B, et al. Effects of erythropoietin on hippocampal volume and memory in mood disorders. Biol Psychiatry. 2015;78:270-7.

31. Hassouna I, Ott C, Wüstefeld L, Offen N, Neher RA, Mitkovski $\mathrm{M}$, et al. Revisiting adult neurogenesis and the role of erythropoietin for neuronal and oligodendroglial differentiation in the hippocampus. Mol Psychiatry. 2016:21:1752-67.

32. Wakhloo D, Scharkowski F, Curto Y, Butt UJ, Bansal V, Steixner-Kumar AA, et al. Functional hypoxia drives neuroplasticity and neurogenesis via brain erythropoietin. Nat Commun. 2020;11:1-12.

33. Kimura W, Xiao F, Canseco DC, Muralidhar S, Thet S, Zhang $\mathrm{HM}$, et al. Hypoxia fate mapping identifies cycling cardiomyocytes in the adult heart. Nature. 2015:523:226-30.

34. Madisen L, Zwingman TA, Sunkin SM, Oh SW, Zariwala HA, Gu $\mathrm{H}$, et al. A robust and high-throughput Cre reporting and characterization system for the whole mouse brain. Nat Neurosci. 2010;13:133.

35. Liebetanz D, Merkler D. Effects of commissural de- and remyelination on motor skill behaviour in the cuprizone mouse model of multiple sclerosis. Exp Neurol. 2006;202:217-24.

36. McKenzie IA, Ohayon D, Li H, Paes de Faria J, Emery B, Tohyama $\mathrm{K}$, et al. Motor skill learning requires active central myelination. Science. 2014;346:318-22.

37. Liebmann T, Renier N, Bettayeb K, Greengard P, Tessier-Lavigne M, Flajolet M. Three-dimensional study of Alzheimer's disease hallmarks using the iDISCO clearing method. Cell Rep. 2016;16: $1138-52$.

38. Lein ES, Hawrylycz MJ, Ao N, Ayres M, Bensinger A, Bernard $\mathrm{A}$, et al. Genome-wide atlas of gene expression in the adult mouse brain. Nature. 2006;445:168-76.

39. Team RC. R: a language and environment for statistical computing. Vienna: R Foundation for Statistical Computing; 2018.

40. Butler A, Hoffman P, Smibert P, Papalexi E, Satija R. Integrating single-cell transcriptomic data across different conditions, technologies, and species. Nat Biotechnol. 2018;36:411-20.

41. Stuart T, Butler A, Hoffman P, Hafemeister C, Papalexi E, Mauck WM III, et al. Comprehensive Integration of single-cell data. Cell. 2019;177:1888-1902.e1821.

42. McGinnis CS, Murrow LM, Gartner ZJ. DoubletFinder: double detection in single-cell RNA sequencing data using artificial nearest neighbors. Cell Syst. 2019;8:329-337 e324.

43. Zeisel A, Muñoz-Manchado AB, Codeluppi S, Lönnerberg P, La Manno G, Juréus A, et al. Cell types in the mouse cortex and hippocampus revealed by single-cell RNA-seq. Science. 2015;347:1138.

44. Dere E, Dahm L, Lu D, Hammerschmidt K, Ju A, Tantra M, et al. Heterozygous AMBRA1 deficiency in mice: a genetic trait with autism-like behavior restricted to the female gender. Front Behav Neurosci. 2014:8:181

45. Ribbe K, Friedrichs H, Begemann M, Grube S, Papiol S, Kästner A, et al. The cross-sectional GRAS sample: A comprehensive phenotypical data collection of schizophrenic patients. BMC Psychiatry. 2010;10:91.

46. Penny JE, Kukums JR, Tyrer JH, Eadie MJ. Selective vulnerability of the hippocampus to hypoxia: cytophotometric studies of enzyme activity in single neurones. Proc Aust Assoc Neurol. $1974 ; 11: 177-81$.

47. Ng T, Graham DI, Adams JH, Ford I. Changes in the hippocampus and the cerebellum resulting from hypoxic insults: frequency and distribution. Acta Neuropathol. 1989;78: $438-43$. 
48. Schmidt-Kastner R. Genomic approach to selective vulnerability of the hippocampus in brain ischemia-hypoxia. Neuroscience. 2015:309:259-79.

49. Sommer W. Erkrankung des Ammonshorns als aetiologisches Moment der Epilepsie. Arch Psychiatr Nervenkr. 1880;10: 631-75.

50. Chatzi C, Schnell E, Westbrook GL. Localized hypoxia within the subgranular zone determines the early survival of newborn hippocampal granule cells. Elife. 2015:4:e08722.

51. Mazumdar J, O'brien WT, Johnson RS, LaManna JC, Chavez JC Klein PS, et al. O2 regulates stem cells through Wnt/ $\beta$-catenin signalling. Nat Cell Biol. 2010;12:1007-13.

52. Power RM, Huisken J. A guide to light-sheet fluorescence microscopy for multiscale imaging. Nat Methods. 2017;14: 360-73.

53. Mathupala SP, Rempel A, Pedersen PL. Glucose catabolism in cancer cells: Identification and characterization of a marked activation response of the type ii hexokinase gene to hypoxic conditions. J Biol Chem. 2001;276:43407-12.

54. Wolf A, Agnihotri S, Micallef J, Mukherjee J, Sabha N, Cairns R, et al. Hexokinase 2 is a key mediator of aerobic glycolysis and promotes tumor growth in human glioblastoma multiforme. J Exp Med. 2011;208:313-26.

55. Brandenburg S, Müller A, Turkowski K, Radev YT, Rot S, Schmidt C, et al. Resident microglia rather than peripheral macrophages promote vascularization in brain tumors and are source of alternative pro-angiogenic factors. Acta Neuropathol. 2016;131:365-78.

56. Pan Y, Mansfield KD, Bertozzi CC, Rudenko V, Chan DA Giaccia AJ, et al. Multiple factors affecting cellular redox status and energy metabolism modulate hypoxia-inducible factor proly hydroxylase activity in vivo and in vitro. Mol Cell Biol. 2007;27:912-25.

57. Guo Y, Wang Z, Prathap S, Holschneider DP. Recruitment of prefrontal-striatal circuit in response to skilled motor challenge. Neuroreport. 2017;28:1187-94.

58. Fabel K, Wolf SA, Ehninger D, Babu H, Leal-Galicia P, Kempermann G. Additive effects of physical exercise and environmental enrichment on adult hippocampal neurogenesis in mice. Front Neurosci. 2009;3:50.

59. Kobilo T, Liu QR, Gandhi K, Mughal M, Shaham Y, van Praag $\mathrm{H}$. Running is the neurogenic and neurotrophic stimulus in environmental enrichment. Learn Mem. 2011;18:605-9.
60. Cuffe JS, Walton SL, Singh RR, Spiers JG, Bielefeldt-Ohmann H, Wilkinson L, et al. Mid- to late term hypoxia in the mouse alters placental morphology, glucocorticoid regulatory pathways and nutrient transporters in a sex-specific manner. J Physiol. 2014;592:3127-41.

61. Lan WC, Priestley M, Mayoral SR, Tian L, Shamloo M, Penn AA. Sex-specific cognitive deficits and regional brain volume loss in mice exposed to chronic, sublethal hypoxia. Pediatr Res. 2011;70:15-20.

62. Soliz J, Thomsen JJ, Soulage C, Lundby C, Gassmann M. Sexdependent regulation of hypoxic ventilation in mice and humans is mediated by erythropoietin. Am J Physiol Regul Integr Comp Physiol. 2009;296:R1837-46.

63. Heyer A, Hasselblatt M, von Ahsen N, Häfner H, Sirén A-L, Ehrenreich $\mathrm{H}$. In vitro gender differences in neuronal survival on hypoxia and in $17 \beta$-estradiol-mediated neuroprotection. J Cereb Blood Flow Metab. 2005:25:427-30.

64. Halliday MR, Abeydeera D, Lundquist AJ, Petzinger GM, Jakowec MW. Intensive treadmill exercise increases expression of hypoxia-inducible factor lalpha and its downstream transcript targets: a potential role in neuroplasticity. Neuroreport. 2019;30:619-27

65. Jögi A, Vallon-Christersson J, Holmquist L, Axelson H, Borg Å, Påhlman S. Human neuroblastoma cells exposed to hypoxia: induction of genes associated with growth, survival, and aggressive behavior. Exp Cell Res. 2004;295:469-87

66. Steinmet7. NA, Zatka-Haas P, Carandini M, Harris KD. Distributed coding of choice, action and engagement across the mouse brain. Nature. 2019;576:266-73.

67. Marques JC, Li M, Schaak D, Robson DN, Li JM. Internal state dynamics shape brainwide activity and foraging behaviour. Nature. 2020;577:239-43

68. Rhodes JS, Garland T Jr, Gammie SC. Patterns of brain activity associated with variation in voluntary wheel-running behavior. Behav Neurosci. 2003;117:1243.

69. Denk F, Ramer LM, Erskine EL, Nassar MA, Bogdanov Y, Signore M, et al. Tamoxifen induces cellular stress in the nervous system by inhibiting cholesterol synthesis. Acta Neuropathol Commun. 2015;3:74.

70. Joshi S, Singh AR, Durden DL. MDM2 regulates hypoxic hypoxia-inducible factor 1alpha stability in an E3 ligase, proteasome, and PTEN-phosphatidylinositol 3-kinase-AKT-dependent manner. J Biol Chem. 2014;289:22785-97. 


\section{SUPPLEMENTARY INFORMATION}

\section{Hippocampal neurons respond to brain activity with functional hypoxia}

Umer Javed Butt ${ }^{15}$, Agnes A. Steixner-Kumar ${ }^{15}$, Constanze Depp ${ }^{25}$, Ting Sun ${ }^{2,3}$,

Imam Hassouna', Liane Wüstefeld', Sahab Arinrad ${ }^{1}$, Matthias R. Zillmann'1, Nadine Schopf ${ }^{1}$, Laura Fernandez Garcia-Agudo', Leonie Mohrmann ${ }^{1}$, Ulli Bode ${ }^{2}$, Anja Ronnenberg', Martin Hindermann'1, Sandra Goebbels², Stefan Bonn ${ }^{3}$, Dörthe M. Katschinski ${ }^{4}$,

Kamilla W. Miskowiak ${ }^{5}$, Klaus-Armin $\mathrm{Nave}^{2 *}$, and Hannelore Ehrenreich ${ }^{1 *}$

${ }^{\natural}$ Shared first authorship

${ }^{1}$ Clinical Neuroscience and ${ }^{2}$ Department of Neurogenetics, Max Planck Institute of Experimental Medicine, Göttingen, Germany Institute of Medical Systems Biology, Center for Molecular Neurobiology,

University Clinic Hamburg-Eppendorf, Hamburg, Germany

${ }^{4}$ Institute for Cardiovascular Physiology, University Medical Center Göttingen, Georg-August-University, Göttingen, Germany

${ }^{5}$ Psychiatric Centre Copenhagen, University Hospital, Rigshospitalet, Copenhagen, Denmark

*Correspondence:

Prof. Hannelore Ehrenreich, MD, DVM

Clinical Neuroscience, Max Planck Institute of Experimental Medicine

Hermann-Rein-Str.3, 37075 Göttingen, GERMANY

Phone +49-551-3899615; Fax +49-551-3899670

E-Mail: ehrenreich@em.mpg.de

Prof. Klaus-Armin Nave, PhD

Neurogenetics, Max Planck Institute of Experimental Medicine

Hermann-Rein-Str.3, 37075 Göttingen, GERMANY

Phone: +49-551-3899754; Fax: +49-551-3899758

E-Mail:nave@em.mpg.de 
Butt et al Supplementary Figure 1
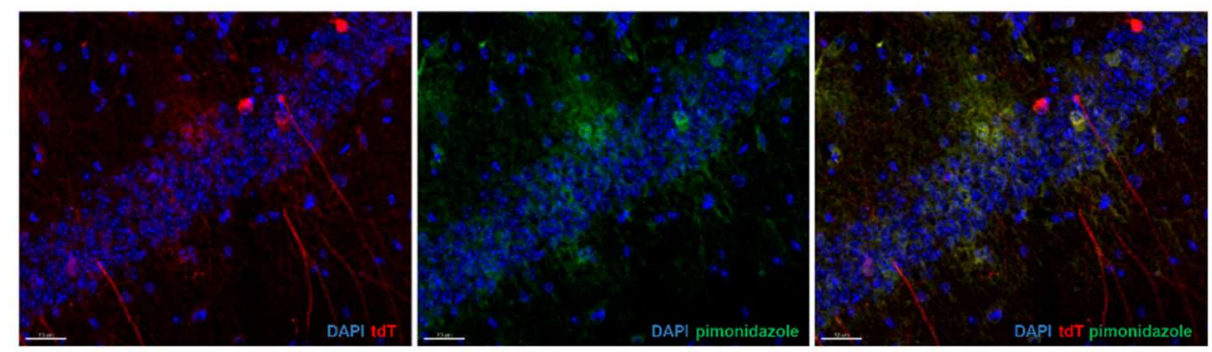

Supplementary Figure 1: Pimonidazole staining confirms functional hypoxia in tdTomato+ cells. Co-labelling of tdTomato and pimonidazole (Sato et al 2011) shows the presence of hypoxia in a subset of tdTomato+ cells (compare Kimura et al 2015). Pimonidazole was administered 16 hours after $1 \mathrm{X}$ tamoxifen injection $(100 \mathrm{mg} / \mathrm{kg}$ i.p.) and CRW start, and mice were sacrificed 90 minutes after pimonidazole administration. Scale bar represents $30 \mu \mathrm{m}$.

\section{References:}

Sato, Y., Endo, H., Okuyama, H., Takeda, T., Iwahashi, H., Imagawa, A., et al. Cellular hypoxia of pancreatic $\beta$-cells due to high levels of oxygen consumption for insulin secretion in vitro. Journal of Biological Chemistry 286, 12524-12532 (2011).

Kimura W, Xiao F, Canseco DC, Muralidhar S, Thet S, Zhang HM et al. Hypoxia fate mapping identifies cycling cardiomyocytes in the adult heart. Nature 2015; 523(7559): 226-230. 
a

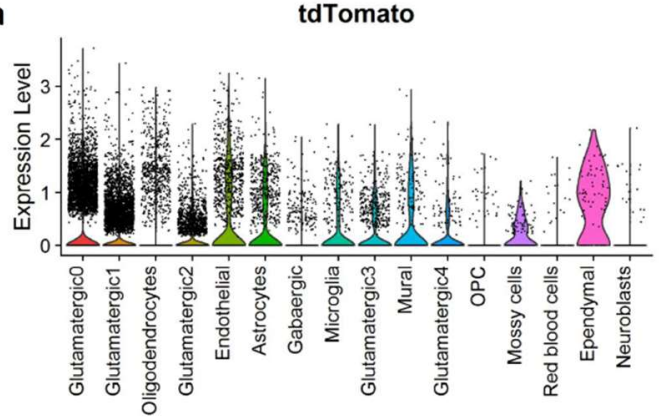

b

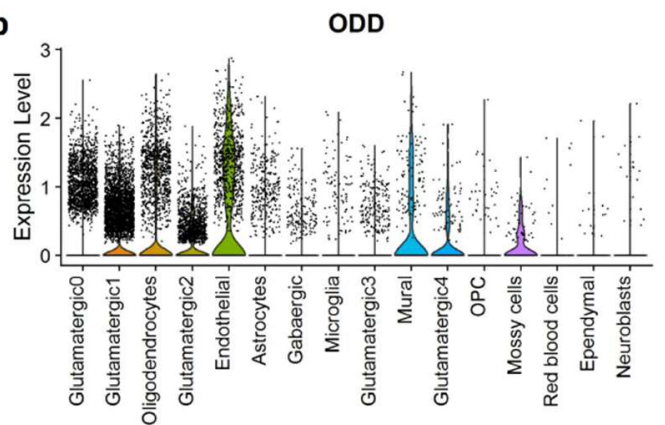

Supplementary Figure 2: Normalized expression levels of (a) tdTomato and (b) ODD in respective cell clusters. No differences in mean expression of either tdTomato or ODD ( $p>0.2$, Wilcoxon test, 2-tailed) were observed between microglia and neurons, i.e. the least (microglia) and the most pronounced (neurons, comprising glutamatergic0-4, gabaergic and mossy cell clusters) hypoxia-labelled cell types. 
Butt et al Supplementary Figure 3a

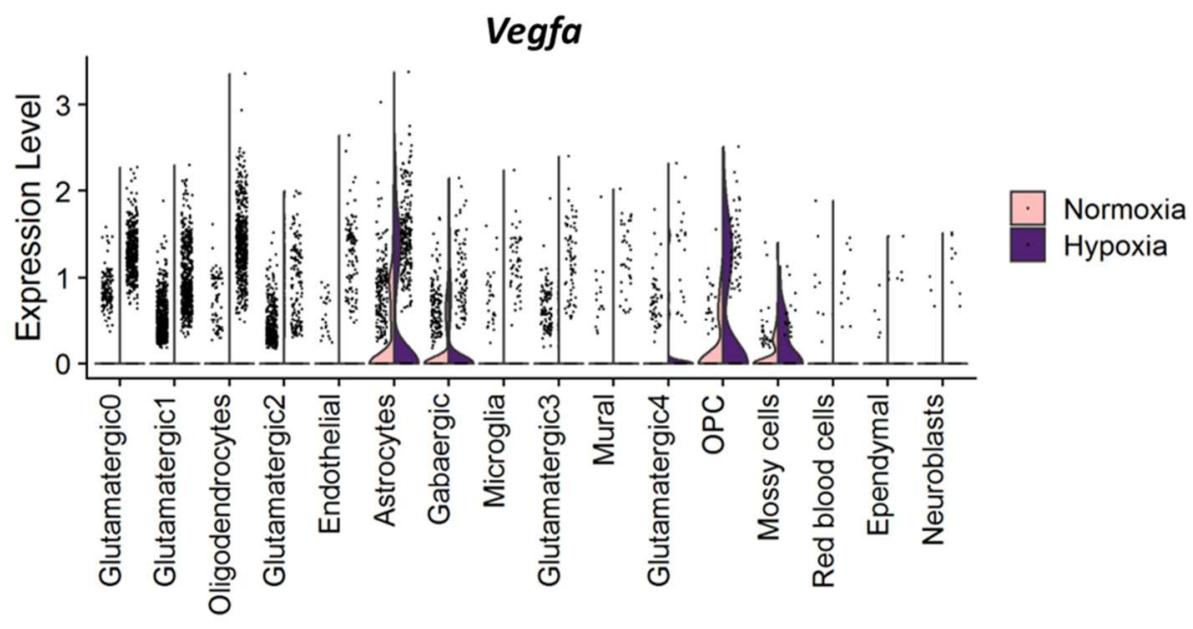

Supplementary Figure 3a: Violin plots showing expression of Vegfa under normoxia and hypoxia in all hippocampal cell populations. Vegfa was highest expressed in OPC and astrocytes (avg_logFC $>0.25, \mathrm{p}_{\text {unadj }}<0.05$ ) when compared to all other cell types. 
Butt et al Supplementary Figure 3b

\begin{tabular}{|l|l|l|l|l|l|}
\hline Cluster & p_val & avg_logFC & $\begin{array}{l}\text { Hypoxia } \\
\text { proportion }\end{array}$ & $\begin{array}{l}\text { Normoxia } \\
\text { proportion }\end{array}$ & p_val adj \\
\hline Glutamatergic0 & $1.06 \mathrm{E}-32$ & 0.221406 & 0.118 & 0.044 & $2.0 \mathrm{E}-28$ \\
\hline Glutamatergic1 & $6.28 \mathrm{E}-04$ & 0.169033 & 0.173 & 0.163 & $1.0 \mathrm{E}+00$ \\
\hline Oligodendrocytes & $4.84 \mathrm{E}-20$ & 0.468132 & 0.228 & 0.092 & $9.2 \mathrm{E}-16$ \\
\hline Glutamatergic2 & $1.27 \mathrm{E}-01$ & 0.205379 & 0.222 & 0.225 & $1.0 \mathrm{E}+00$ \\
\hline Endothelial & $3.62 \mathrm{E}-03$ & 0.211827 & 0.101 & 0.057 & $1.0 \mathrm{E}+00$ \\
\hline Astrocytes & $1.68 \mathrm{E}-05$ & 0.470057 & 0.374 & 0.35 & $3.2 \mathrm{E}-01$ \\
\hline Gabaergic & $8.91 \mathrm{E}-01$ & 0.154432 & 0.258 & 0.293 & $1.0 \mathrm{E}+00$ \\
\hline Microglia & $6.40 \mathrm{E}-01$ & 0.09817 & 0.153 & 0.188 & $1.0 \mathrm{E}+00$ \\
\hline Glutamatergic3 & $3.30 \mathrm{E}-03$ & 0.313916 & 0.225 & 0.171 & $1.0 \mathrm{E}+00$ \\
\hline Mural cells & $2.00 \mathrm{E}-01$ & 0.049427 & 0.129 & 0.203 & $1.0 \mathrm{E}+00$ \\
\hline Glutamatergic4 & $4.94 \mathrm{E}-01$ & 0.317821 & 0.25 & 0.246 & $1.0 \mathrm{E}+00$ \\
\hline OPC & $6.77 \mathrm{E}-03$ & 0.531906 & 0.389 & 0.293 & $1.0 \mathrm{E}+00$ \\
\hline Mossy cells & $4.25 \mathrm{E}-02$ & 0.141386 & 0.417 & 0.293 & $1.0 \mathrm{E}+00$ \\
\hline Red blood cells & $6.89 \mathrm{E}-01$ & -0.05511 & 0.108 & 0.129 & $1.0 \mathrm{E}+00$ \\
\hline Ependymal cells & $1.92 \mathrm{E}-01$ & 0.255091 & 0.174 & 0.086 & $1.0 \mathrm{E}+00$ \\
\hline Neuroblasts & $4.49 \mathrm{E}-01$ & 0.141355 & 0.116 & 0.075 & $1.0 \mathrm{E}+00$ \\
\hline & & & & & \\
\hline
\end{tabular}

Supplementary Figure 3b: Differential expression testing for Vegfa (as shown in Figure 4a) under hypoxia versus normoxia in all cell types (Wilcoxon test, 2-tailed); avg_logFC: positive log fold-change values indicate upregulation under hypoxia, negative values downregulation under hypoxia. Hypoxia proportion: Proportion of cells expressing Vegfa under hypoxia; Normoxia proportion: Proportion of cells expressing Vegfa under normoxia. P_val_adj: Bonferroni adjusted p-value (adjusted for all genes in data set). 
Butt et al Supplementary Figure 4a

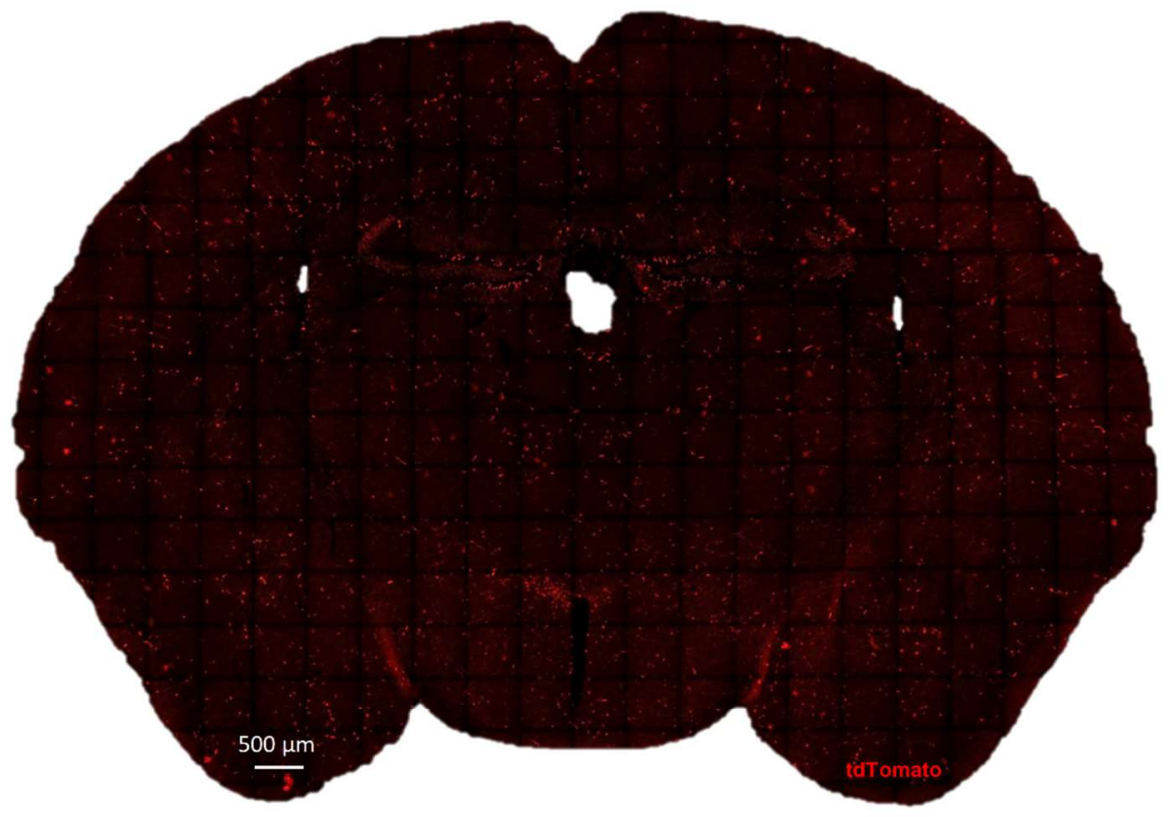

Supplementary Figure 4a: Representative coronal brain section of a CAG-CreERT2-

ODD::R26R-tdTomato mouse shows brain-wide distribution of scattered tdTomato+ cells under normoxia ( $5 \mathrm{X}$ tamoxifen injections). 
Butt et al Supplementary Figure 4b

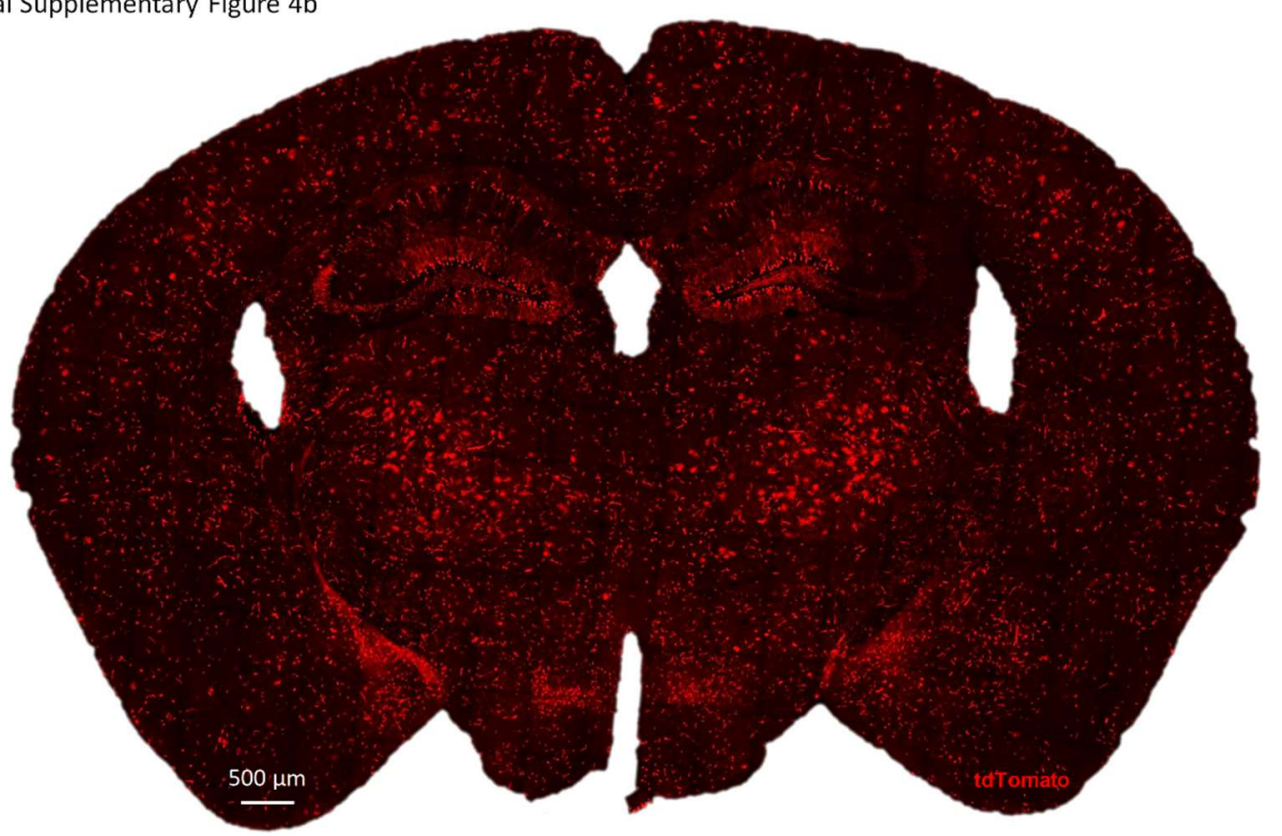

Supplementary Figure 4b: Representative coronal brain section of a CAG-CreERT2ODD::R26R-tdTomato mouse shows globally enhanced numbers and intensity of tdTomato+ cells after exposure to CRW (5 consecutive nights, $5 \mathrm{X}$ tamoxifen injections). The widely distributed tdTomato labelling indicates brain-wide response to motor-cognitive challenge. 


\subsection{Chapter II. Further experimental data on the CAG-CreERT2- ODD::R26R-tdTomato line}

The first part of chapter II mainly focuses on the results obtained from the CA1 layer of the hippocampus. However, in the framework of this project we obtained results using the CAG-CreERT2-ODD::R26R-tdTomato mice in various other experiments, which were not included in the manuscript. These results are reported and discussed in the following.

Tamoxifen dose-response curve: The response to different tamoxifen doses was evaluated in CAG-CreERT2-ODD::R26R-tdTomato mice as shown in figure 1. Mice received (a) $1 X$, (b) $3 X$ and (c) $5 X$ tamoxifen injections, along with hypoxia $\left(6 \% \mathrm{O}_{2}\right)$ for $6 \mathrm{~h}$. In a modified treatment paradigm, mice received 3-tamoxifen injections and were exposed to hypoxia $\left(6 \% \mathrm{O}_{2}\right.$ for $\left.6 \mathrm{~h}\right)$ for 5 consecutive days (Figure 1b). 2 days after the last injection mice were sacrificed and brains were collected for quantification of neurons. In the CA1 ( $1 X p=0.02,3 X p=0.03,3 X 5 X$ $p=0.02$ and $5 \times 5 X p=0.04), C A 3(1 X p=0.014,3 X p=0.01,3 X 5 X p=0.01$ and $5 X 5 X$ $p=0.03)$ and the DG (1X $p=0.1,3 X p=0.007,3 \times 5 X p=0.01$ and $5 \times 5 X p=0.007)$ of the hippocampus, quantification of neurons revealed a tamoxifen-dose-dependent increase in the number of $\mathrm{tdT}+/ \mathrm{NeuN}+$ hypoxic neurons under hypoxia as compared to normoxic controls. Hypoxic neurons also increased dramatically in mice receiving the above-mentioned $3 \times 5 X$ treatment paradigm. Overall, in CA1, CA3 and DG of the hippocampus the total number of hypoxic neurons increased proportional to the number of tamoxifen injections and days of hypoxia application.

Oxygen dose-response curve: The impact of hypoxia was studied in CAGCreERT2-ODD::R26R-tdTomato mice by exposing them to variable levels of exogenous hypoxia $\left(20.9 \%, 15 \%, 12 \%, 10 \%, 8 \%\right.$ and $\left.6 \% \mathrm{O}_{2}\right)$ (Figure 2a). Quantification results revealed an increase in the number of tdT+/NeuN+ hypoxic neurons in the CA3 $(p=0.001)$ and the DG $(p=0.003)$ of the hippocampus upon decreasing the oxygen concentration (Figure $2 b$ ). The highest increase was observed at $6 \% \mathrm{O}_{2}$ in comparison to normoxic controls. Moreover, the total number of $\mathrm{tdT}+/ \mathrm{S} 100 ß+$ increased upon decreasing the oxygen concentration in the whole hippocampus $(p=0.0032)$. 
Persistence of tdTomato fluorescent labeling: over time was observed in the CAG-CreERT2-ODD::R26R-tdTomato mice as shown in figure 3a. Mice received 5 tamoxifen injections and were kept under normoxic conditions. Brains were collected at different time points post treatment (3days, 1 week, 2 weeks, and 4 weeks). Quantification results in the CA3 region indicate prominent upregulation over the period of 1 month $(p=0.056)$ (Figure $3 b)$. In the $D G$, the total number of td $\mathrm{T}+\mathrm{NeuN}+$ hypoxic neurons was not significantly different $(\mathrm{p}=0.3)$ compared to respective controls (Figure $3 b$ ). In the whole hippocampus, the number of $\operatorname{td} T+/ S 100 ß+$ astrocytes was also not significantly different from controls $(p=0.3)$ (Figure 3c).

Exercise and motor learning-induced hypoxia was checked in CAGCreERT2-ODD::R26R-tdTomato mice (4 weeks age) as shown in figure 4a. In brief, mice received 5-tamoxifen injections along with exposure to CRW or hypoxia $\left(6 \% \mathrm{O}_{2}\right)$ or normoxia $\left(21.9 \% \mathrm{O}_{2}\right)$. Quantification results indicate an increase in hypoxic neurons in the CA3 (males $p=0.0001$, females $p=0.0001$ ) and DG (males $p=0.001$, females $p=0.0001$ ) upon exposure to hypoxia. Exposure to CRW did not lead to a significant increase in the CA3 and DG (Figure 4b). The total number of $t d T+/ S 100 ß$ hypoxic neurons increase only upon exposure to hypoxia in both genders (males $p=0.05$, females $p=0.01$ ). However, the total number was significantly different only in females in response to CRW compared to normoxic controls $(p=0.05)$. These results indicate that, the number of hypoxic neurons and astrocytes increases upon hypoxia and CRW exposure in comparison to normoxia.

In 24-week-old mice a similar treatment paradigm was used as explained above (Figure 4a). Quantification results indicate an increase in hypoxic neurons in both genders in CA3 (males $p=0.01$, females $p=0.0001$ ) and DG (males $p=0.07$, females $p=0.001$ ) on hypoxia exposure. The number of $t d T+/ N e u N+$ was not significantly upregulated in mice exposed to voluntary running on CRW compared to normoxic controls. Taken together, these results indicate that hypoxia and CRW lead to an increase in the number of hypoxic cells in the hippocampus representing enhanced oxygen requirement upon activity. 
NRW and CRW: The effect of exercise and motor learning-induced hypoxia in normal and complex running wheel was compared in CAG-CreERT2ODD::R26R-tdTomato male mice (4 weeks age). Mice were exposed to hypoxia, normoxia, complex running wheel (CRW) or normal running wheel (NRW) as shown in figures $5 a$ and $b$. Quantification results indicate an increase in the number of $t d+/ N e u N+$ hypoxic neurons upon exposure to NRW, CRW and hypoxia in the CA1 $(p=0.004), C A 3(p=0.0001)$ and DG $(p=0.02)$. There was an increased tendency in total number of hypoxic neurons in CA1 of CRW vs NRW exposed mice. The number of $\mathrm{td} T+/ \mathrm{S} 100 ß$ hypoxic astrocytes was not statistically different between groups. These results indicate that number of hypoxic neurons increase upon exposure to exercise and challenging motor learning especially in the CA1 and DG.

Hypoxic cells in peripheral organs: As CAG is ubiquitously expressed, peripheral organs from CAG-CreERT2-ODD::R26R-tdTomato mice exposed to hypoxia vs normoxia control, were also investigated. In the kidney, a high number of hypoxic cells was observed under hypoxia compared to normoxic controls (Figure 6). Similarly, in the section from liver, hypoxic cells were observed under both hypoxia and normoxia. These results indicate the sensitivity and proper functioning of the transgene in peripheral organs as shown by tdTomato+ hypoxic cells.

In summary, these results indicate that the number of hypoxic neurons and astrocytes increases upon exposure to exogenous hypoxia. The quantification results of the CA3 and DG are similar to the CA1 region, as reported previously in part 1. Exposure to NRW and CRW leads to an increased number of hypoxic cells in the hippocampus, reflecting the exercise and learning-induced functional hypoxia. Analysis of peripheral organs of CAG-CreERT2-ODD::R26R-tdTomato mice, also revealed hypoxic cell populations in the liver and the kidney. 
a

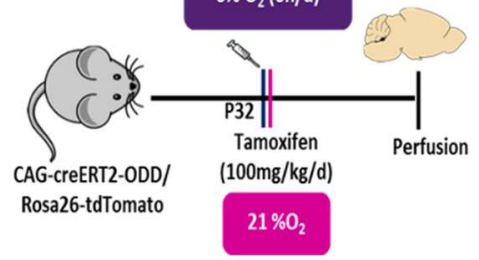

d

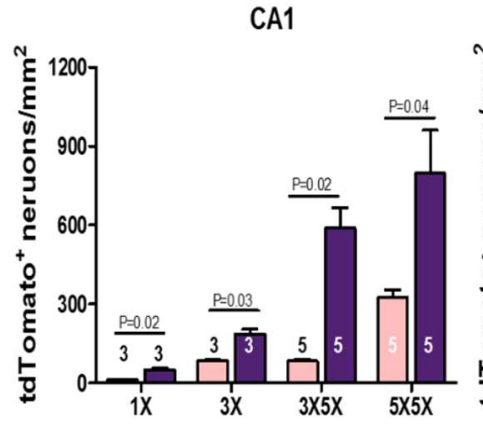

b

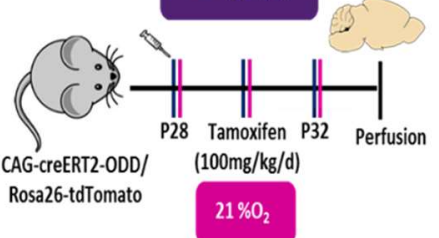

$6 \% \mathrm{O}_{2}(6 \mathrm{~h} / \mathrm{d})$

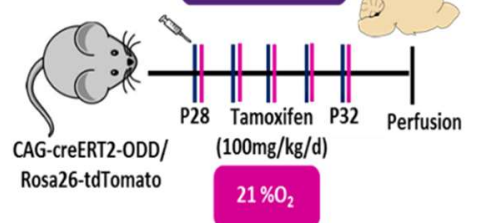

Figure 1. Tamoxifen dose-response curve to characterize CAG-CreERT2-ODD mice (mix gender, 4 weeks). Schematic representation of the tamoxifen dose-response curve treatment. Mice received (a) 1X, (b) 3X or (c) 5X tamoxifen injections, along with $6 \% \mathrm{O}_{2}$ for $6 \mathrm{~h}$ hypoxia exposure every day. All mice were sacrificed and perfused 2 days after the respective last injection. Quantification results indicate a dose-dependent increase in cre induced labelling in the number of hypoxic neurons in the hippocampus. There was a significant increase in the amount of tdTomato+ neurons in the CA1, CA3 and DG between mice undergoing hypoxia vs normoxic controls. Unpaired student t-test (two tailed, Welch's corrections) was used for group comparisons. Error bars indicate standard error of the mean (SEM) and ${ }^{*} P<0.05$, ${ }^{* *} P<0.001$ was considered significant. 


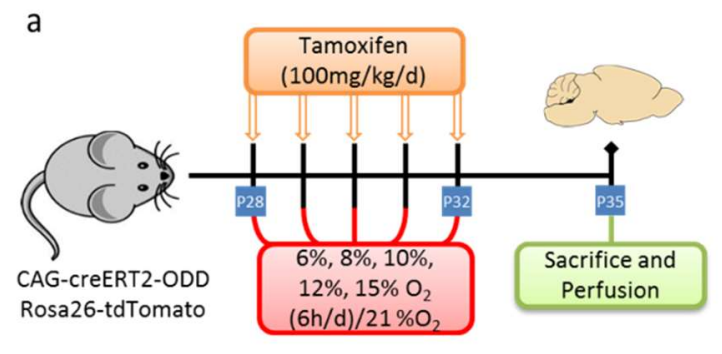

b

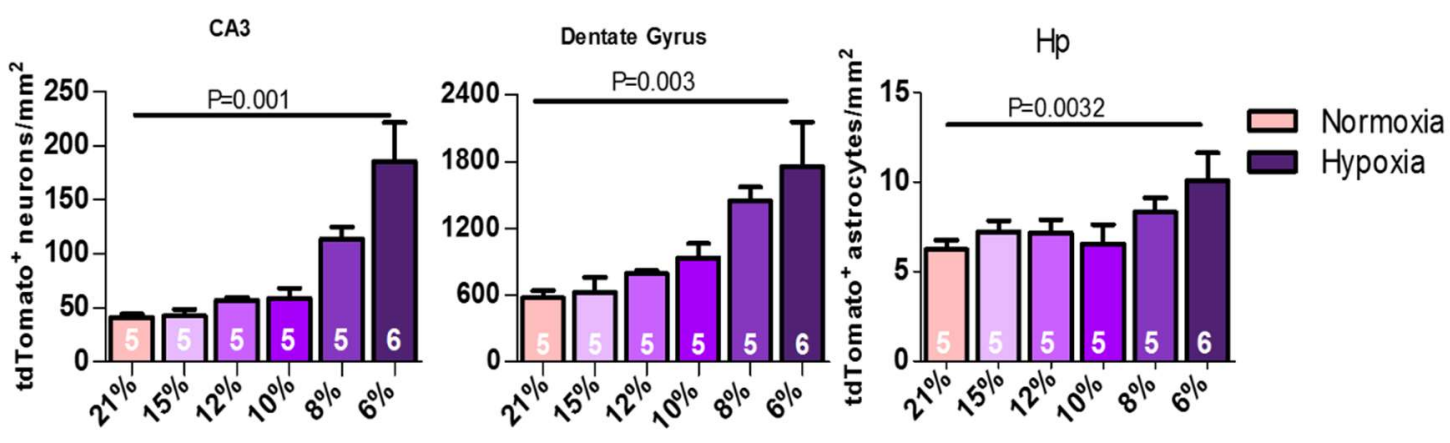

Figure 2. Oxygen-dose-response effect on hypoxic neurons in CAG-CreERT2ODD mice (mix gender, 4 weeks). Schematic representation of the oxygen doseresponse curve treatment. Mice received (a) 5 -tamoxifen injections along with variable hypoxic exposure $\left(6,8,10,12\right.$ and $\left.15 \% \mathrm{O}_{2}\right)$ and control normoxia mice were kept in normal ambient oxygen concentration $\left(21 \% \mathrm{O}_{2}\right)$. Quantification results indicate that dose-dependent increase in cre-induced labelling in number of hypoxic neurons upon decreasing oxygen concentration (b). The total tdTomato+ astrocytes also showed an increase in upon decreasing oxygen concentration. One-Way ANOVA was used for statistical analysis between groups. Error bars indicate standard error of mean (SEM) and ${ }^{*} \mathrm{P}<0.05,{ }^{* *} \mathrm{P}<0.001$ was considered significant. 


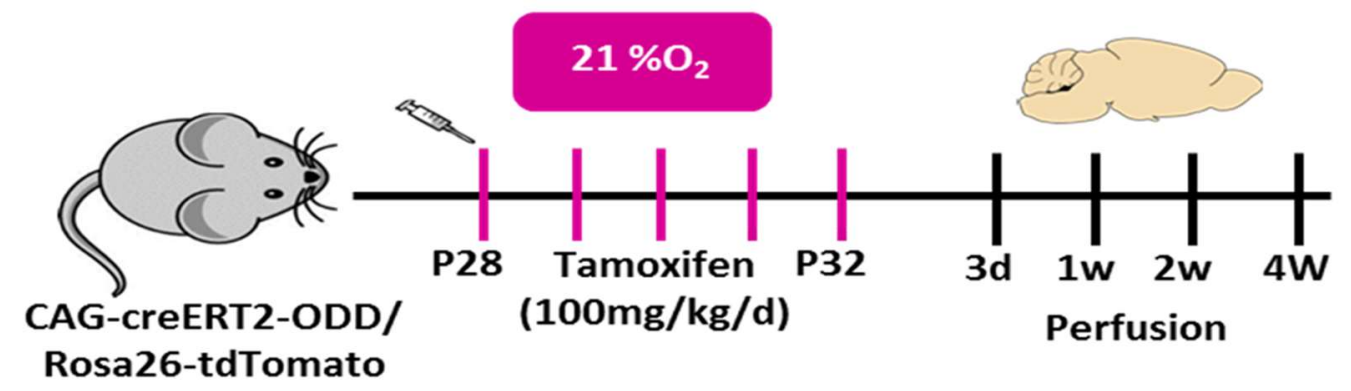

b

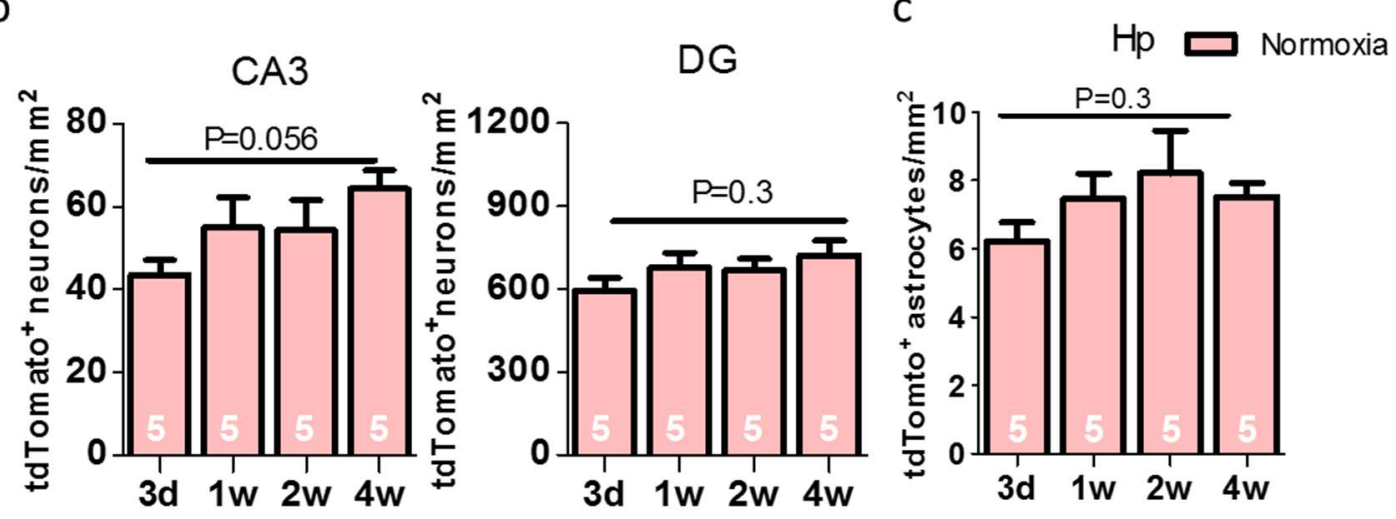

Figure 3. Persistence of tdTomato labelling in CAG-CreERT2-ODD::R26RtdTomato transgenic mice (mix gender, 4 weeks). Schematic representation of the treatment paradigm. Mice received (a) 5 -tamoxifen injections under normoxic conditions $\left(21 \% \mathrm{O}_{2}\right)$ and were sacrificed at different time points post treatment (3days, 1 week, 2 weeks and 4 weeks). Image analysis revealed presence of tdTomato+ neurons even 4 weeks after tamoxifen administration. The total number of tdTomato+ hypoxic neurons (b) and astrocytes (c) was not significantly different between different time points. Hippocampus ( $\mathrm{Hp}$ ). One-Way ANOVA was used for statistical analysis. Error bars indicate standard error of mean (SEM) and ${ }^{*} \mathrm{P}<0.05$, ${ }^{* *} \mathrm{P}<0.001$ was considered significant. 
a

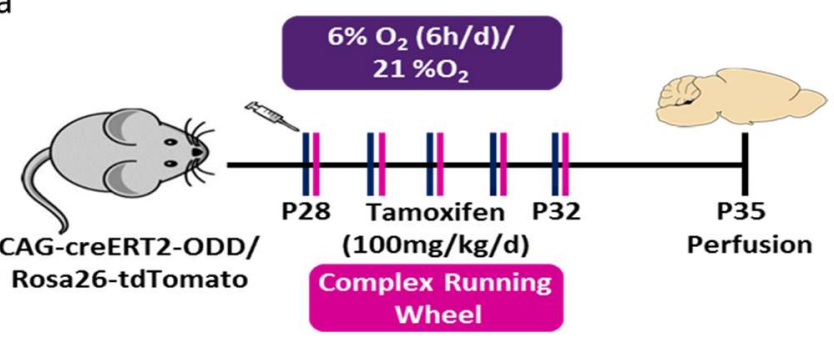

b

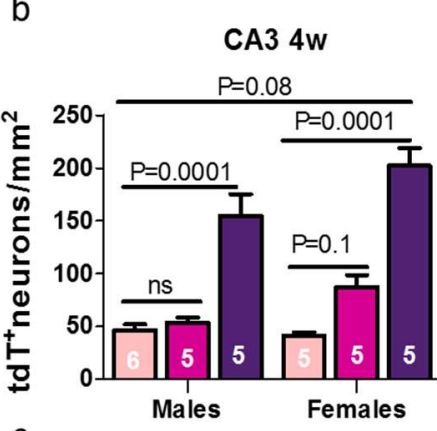

C CA3_24weeks

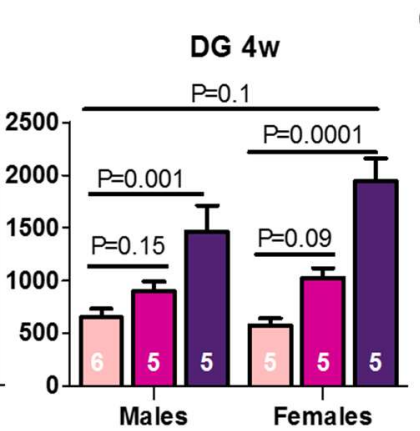

C Hp 4w
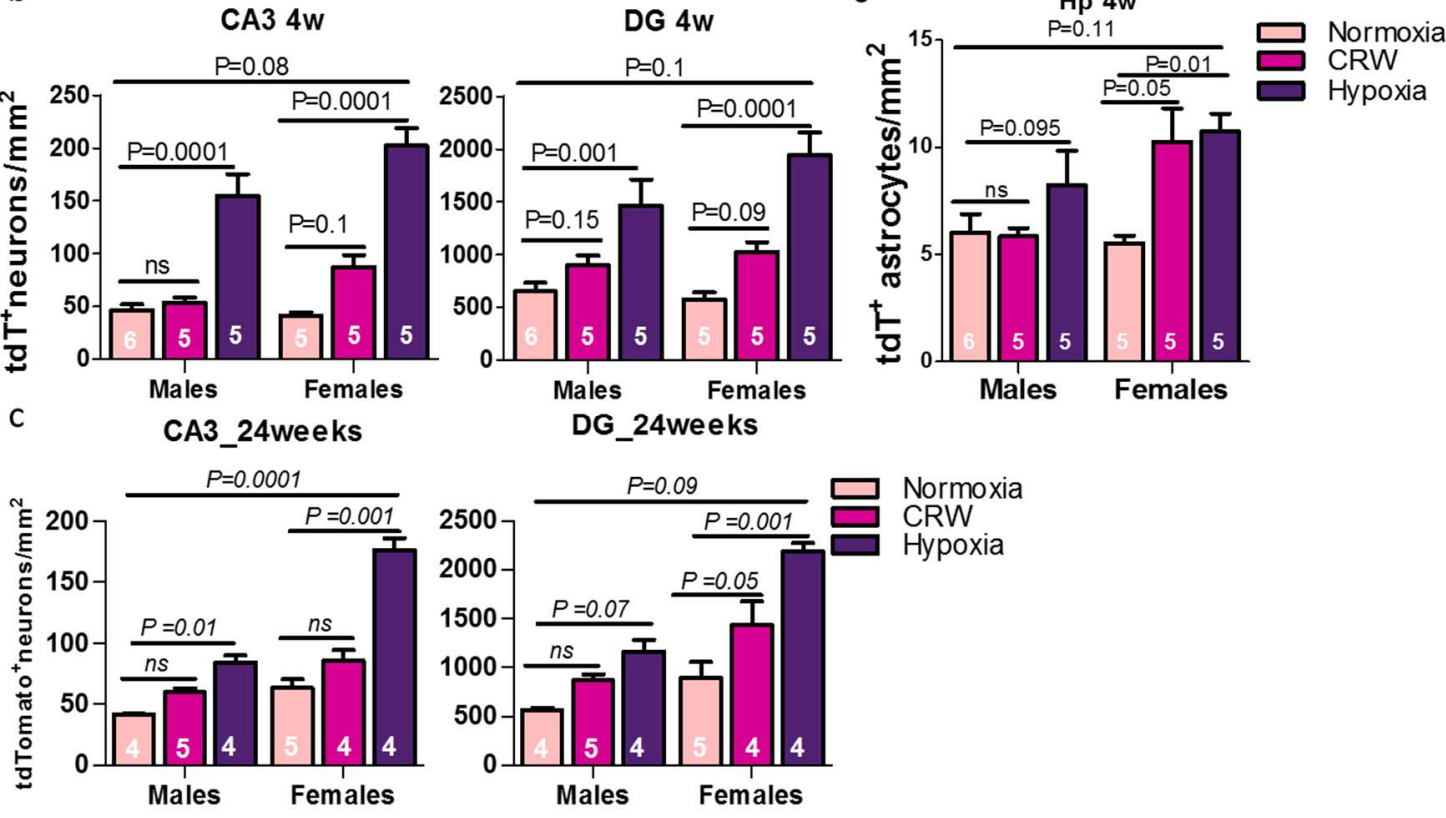

Figure 4. Exercise and motor learning induced hypoxia in the brain (4 and 24 weeks). (a) Schematic diagram of voluntary complex wheel running as an inducer of functional hypoxia in the brain. CAG-CreERT2-ODD::R26R-tdTomato transgenic mice (4 and 24 weeks age) of both genders were included in the experiment. Mice with no prior training were given free access to complex running wheels (CRW) for 5 complete days and received tamoxifen injections every other day. Normoxia control mice received the same treatment but were kept single-housed in normal standard cages. All animals were sacrificed on day 8 of the experiment and brains were collected for histological quantifications. (b) In the CA3 and DG region, the population of $\mathrm{tdT}+/ \mathrm{NeuN}+$ neurons was upregulated in comparison to normoxic controls. Females showed an enhanced response to hypoxia and CRW compared to males. (c) Number of tdT+/S100 $\beta$ cells also increased upon hypoxia and CRW exposure in females as compared to males. Two-way ANOVA with Bonferroni's multiple corrections) was used for statistical analysis. Error bars represent standard error of mean (SEM) and ${ }^{*} \mathrm{P}<0.05,{ }^{* *} \mathrm{P}<0.001$ was considered significant. 
a

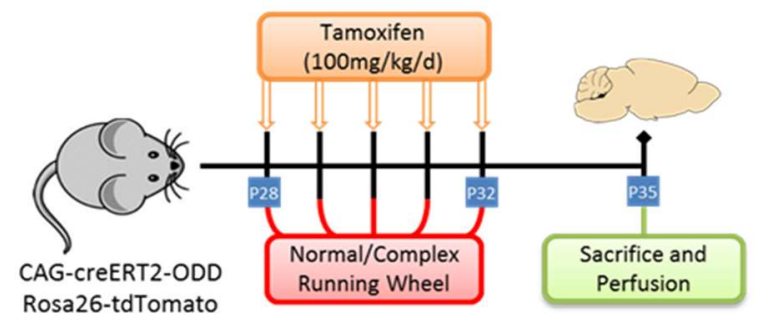

b

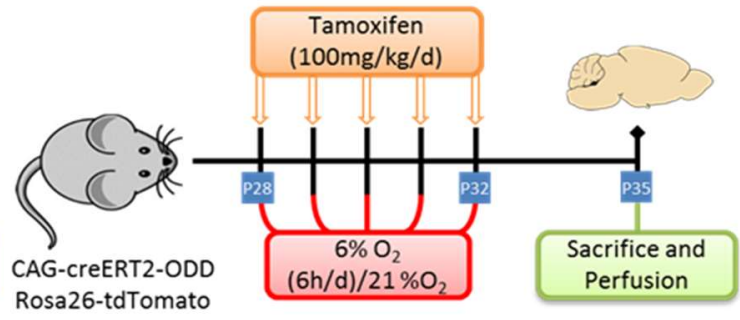

C

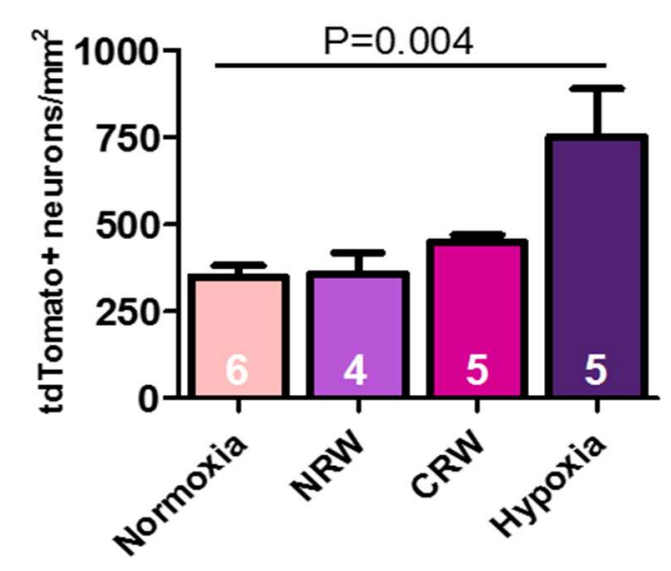

d

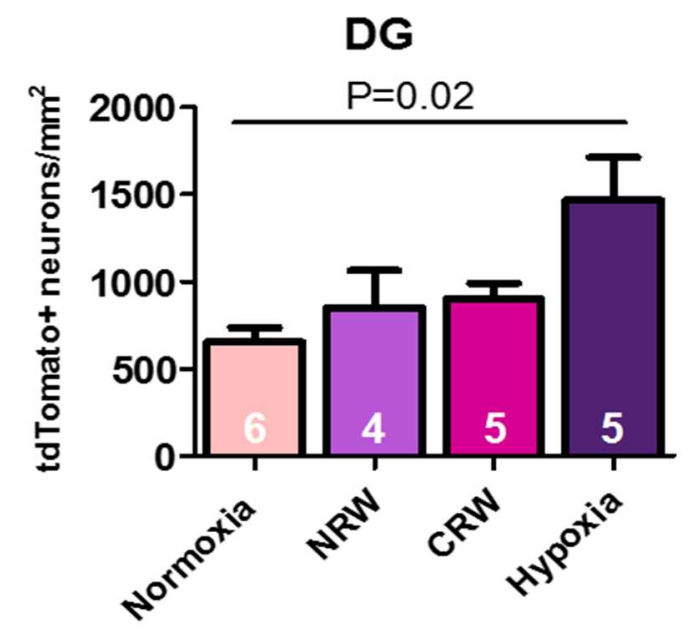

CA3

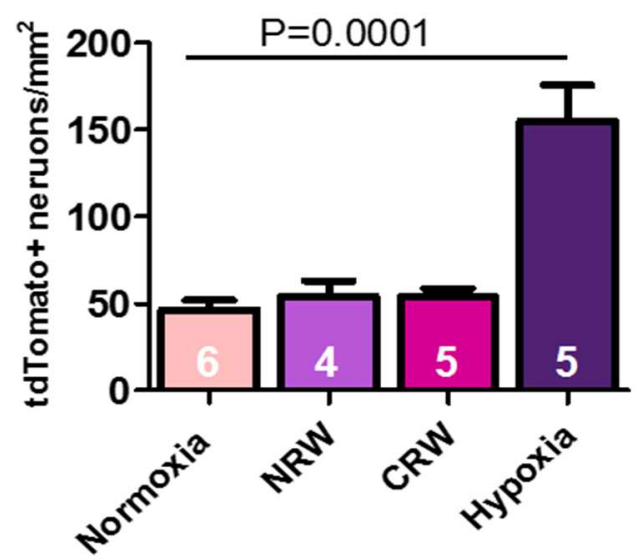

e

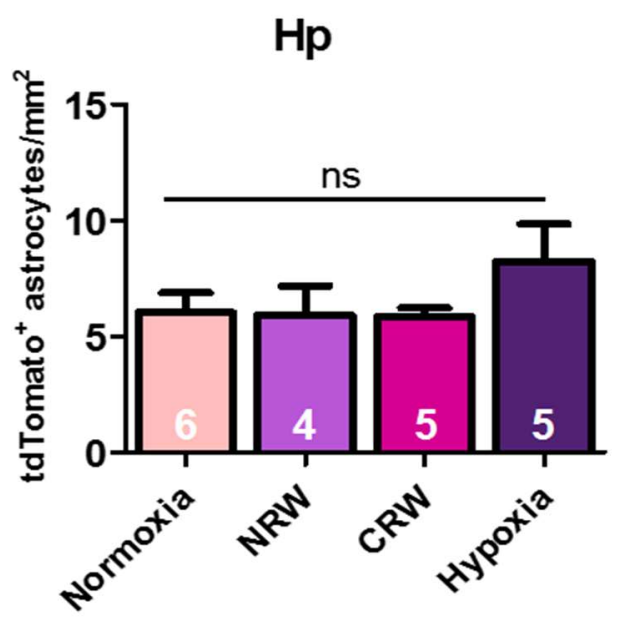

Figure 5. Comparison of complex vs normal running wheels for exercise and motor learning induced hypoxia in the brain (males, 8 weeks). Schematic diagram of voluntary wheel running as an inducer of functional hypoxia in male mice is shown in (a) and for normoxia vs hypoxia treatment in (b). The number of hypoxic neurons increased in the CA1, CA3 and DG in response to the respective treatments (c, d). Overall, the number of hypoxic astrocytes did not change significantly across groups (e). One-way ANOVA with Bonferroni's multiple corrections was used for statistical analysis. Error bars represent standard error of mean (SEM) and ${ }^{*} \mathrm{P}<0.05$, ${ }^{* *} \mathrm{P}<0.001$ was considered significant. 

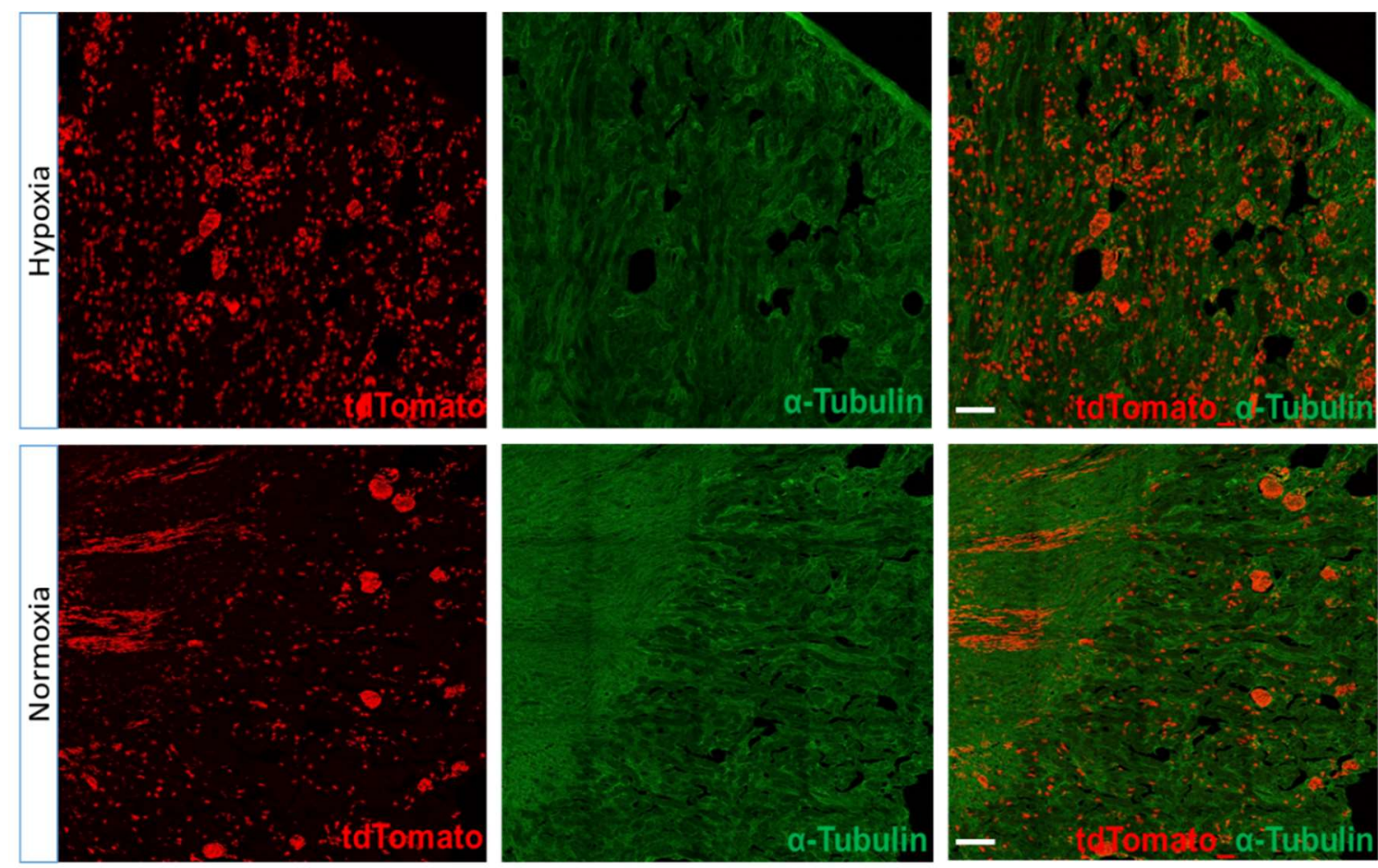

Figure 6. tdTomato+ hypoxic cells in the kidney of CAG-CreERT2-ODD::R26RtdTomato mouse. Kidney sections $(30 \mu \mathrm{m})$ of mice treated according to figure $5 \mathrm{~b}$ were immuno-stained with $\alpha$-Tubulin and illustrative images of normoxia and hypoxia treated mice are shown. Scale bar represents $10 \mu \mathrm{m}$. tdTomato (red), a-Tubulin (green).
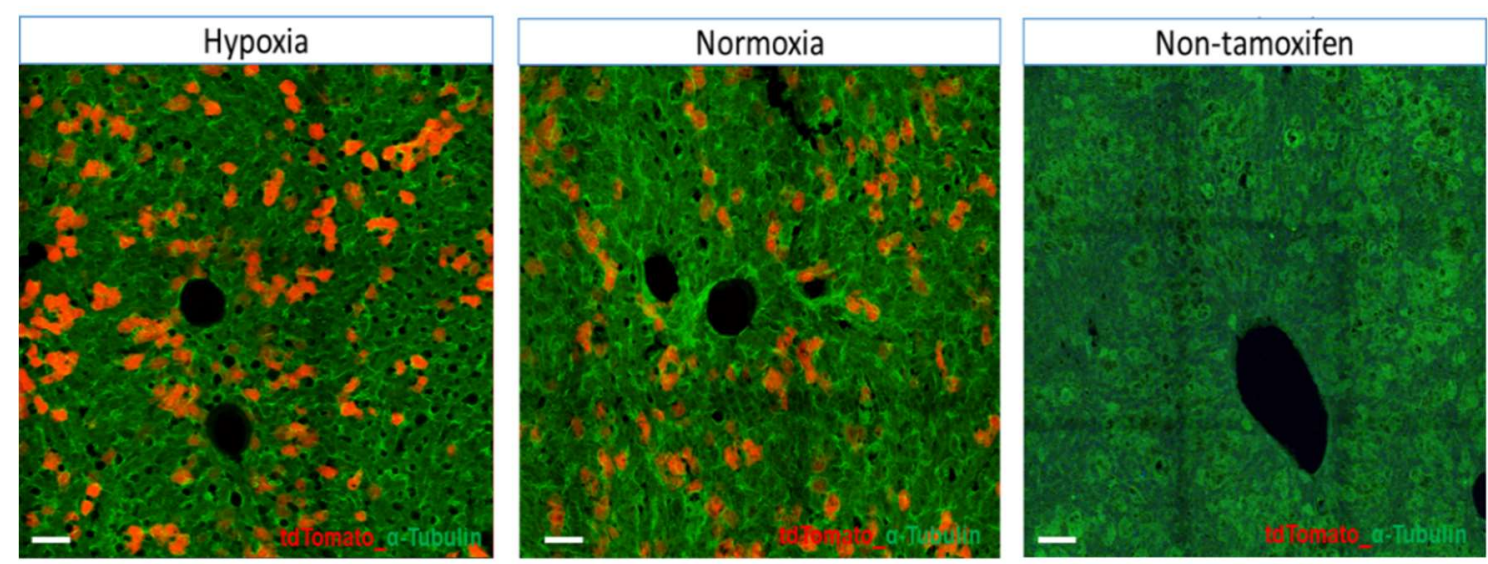

Figure 7. tdTomato+ hypoxic cells in the liver of CAG-CreERT2-ODD::R26RtdTomato mouse. Liver sections $(30 \mu \mathrm{m})$ of mice treated according to treatment shown in (figure $5 b$ ) were immuno-stained with $\alpha$-Tubulin and illustrative images of hypoxia, normoxia and non-tamoxifen treated mice are shown. Scale bar represents $10 \mu \mathrm{m}$. 


\section{Chapter III. Imaging hypoxic neurons with CaMKIla-based and inducible cre-dependent tdTomato reporter mice}

\subsection{Summary of Chapter III}

In the previous Chapter, the generation and characterization of hypoxia sensing CAG-CreERT2-ODD transgenic mice was described. These transgenic mice express inducible cre-recombinase fused with the ODD domain of HIF-1 $\alpha$ under the control of ubiquitous CAG promoter. Upon crossing with R26R-tdTomato reporter mice and tamoxifen administration, all hypoxic cells are irreversibly marked with tdTomato fluorescent protein. Under normal physiological conditions, we observed multiple types of brain cells including neurons, astrocytes, oligodendrocytes, pericytes and endothelial cells undergoing hypoxia. We also examined the consequence of exercise and learning on the oxygen levels in the brain and observed an overall increase in the total number of hypoxic cells. Quantification of tdTomato+ neurons in the dorsal or rostral hippocampus of CRW mice indicates a modest increase in the number of hypoxic neurons in comparison to the normoxic condition. 3-Dimensional visualization of all hypoxic cells by LSM demonstrated global distribution in the whole-brain. The total number of hypoxic cells increased upon application of exogenous hypoxia as well as exposure to $\mathrm{CRW}$ in comparison to normoxic condition. Overall, we observed that the neuronal population was abundantly labelled with tdTomato fluorescent protein throughout the entire brain, highlighting the susceptibility of neurons to hypoxic challenges.

Previously, we confirmed the occurrence of functional hypoxia with the use of global hypoxia reporter mice expressing a CAG promoter. In order to visualize exercise and learning-induced hypoxia only in the neurons, irrespective of other cell types, we developed a new hypoxia reporter mouse restricted to the excitatory neuronal populations.

CaMKIla-CreERT2-ODD-pA transgenic mice: The aim of this project was to develop new transgenic hypoxia-reporting mice, allowing spatial and temporal mapping of low oxygen concentration only in a restricted neuronal population of the brain. For this purpose, the CaMKIla promoter was selected due to its high expression in excitatory pyramidal neurons and their role in learning and memory. 
The expression of CaMKIla is developmentally regulated and reaches its maximum after postnatal week 3 (Coultrap et al., 2012; Mayford et al., 1996). In order to generate a construct expressing inducible cre-recombinase fused with oxygendependent degradation (ODD) domain of HIF-1a driven by CaMKIla promoter, first the CAG promoter was excised from pCAG-CreERT2-ODD-pA (Kimura et al., 2015). Subsequently, the CaMKIla sequence was introduced into the CreERT2-ODD-pA backbone to assemble CaMKIla-CreERT2-ODD-pA. In the next step, transgenic mice expressing CaMKIla-CreERT2-ODD-pA were generated by microinjection.

In order to label and visualize HIF-1 $\alpha$ expressing hypoxic cells in the brain, CaMKIla-CreERT2-ODD transgenic mice were bred to R26R-tdTomato reporter mice. F2 litters harboring the CaMKIla-CreERT2-ODD::R26R-tdTomato transgene were selected for an initial screening characterization of the transgenic mice. First, the oxygen-dependent regulation of the transgene was tested by the application of exogenous hypoxia $\left(6 \% \mathrm{O}_{2}\right)$ and under ambient oxygen concentration by tamoxifen administration. Control mice only received vehicle (oil) injections. Brains were harvested, sectioned and visualized under the confocal microscope for a native tdTomato+ fluorescent protein signal. In addition, not only distinct labelling of hypoxic neurons with tdTomato+ fluorescent signal was observed in hypoxic conditions but also under normoxic conditions throughout the brain. Interestingly, hypoxic neurons were observed under normal physiological conditions. This is in line with the results observed in the CAG-CreERT2-ODD::R26RtdTomato mice. On the other hand, analysis of tamoxifen-independent-induction and labelling of neurons revealed minimal background noise in the mice.

Tamoxifen dose-response curve: In the proof of principle experiment based on previous findings in the CAG-CreERT2-ODD::R26R-tdTomato, different tamoxifen administration regimens were tested in CaMKIla-CreERT2-ODD::R26RtdTomato mice. This experiment assessed the working and labelling efficacy of CaMKIla promoter expression in different areas of the brain. Therefore, transgenic mice received $1 \mathrm{X}, 3 \mathrm{X}$ or $5 \mathrm{X}$ injections along with intermittent hypoxia for respective days for $6 \mathrm{~h}$ at $6 \% \mathrm{O}_{2}$ daily. Mice were sacrificed on day 8 and the brains were harvested. Quantification results confirmed that the number of hypoxic neu- 
rons in CA1, CA3 and DG of dorsal hippocampus increased upon tamoxifen injections. Tamoxifen dose-response curve results indicate a steady increase in number of hypoxic cells. 5X tamoxifen injections produced excessive amounts of cre-induced tdTomato labelling in the brain, which hampered the quantification of individual neurons especially in the dentate gyrus (DG). Thus, for further experiments, a 3-tamoxifen injection paradigm on every other day was considered suitable.

Cellular characterization of hypoxic neurons: Next, we characterized and confirmed the neuronal phenotype of tdTomato+ cells in the CaMKIla-CreERT2ODD::R26R-tdTomato mice. Coronal brain sections $(30 \mu \mathrm{m})$ were stained and immuno-labelled with neuron specific markers (NeuN, Ctip2) followed by visualization under the confocal microscope. tdTomato+ cells overlapping with NeuN signals were observed throughout the brain. Distribution of hypoxic neurons in CaMKIla-CreERT2-ODD::R26R-tdTomato mice were confirmed in hippocampus, cortex, striatum, olfactory bulb and prefrontal cortex. Studies involving mRNA and protein level have also reported a similar distribution of CaMKIla promoter expression (Burgin et al., 1990; Mayford et al., 1996; Wang et al., 2013). The highest number of CaMKIla expressing cells were detected in the dentate gyrus and an overall even distribution in different layers of cortex was observed. We did not see tdTomato signal in other cell types upon tamoxifen-induction, which showed the neuron-specific expression of the introduced transgene.

Learning-induced hypoxia: To test our hypothesis that challenging learning and cognitive tasks lead to transient episodes of functional hypoxia, we used a voluntary running wheel task to assess the effects in CaMKIla-CreERT2-ODD::R26RtdTomato mice. Exercise and motor activity-induced changes in the cells have been discussed in detail in the introduction. We have previously demonstrated that running naïve mice upon exposure to normal and complex running wheel for a period of 5 days leads to a significant increase in the number of hypoxic neurons in the hippocampus in CAG-CreERT2-ODD::R26R-tdTomato mice. A similar experimental paradigm was tested in CaMKIla-CreERT2-ODD::R26R-tdTomato mice with 3-tamoxifen injections on 5 consecutive days. Subsequently, dorsal or rostral hippocampal hypoxic neurons were quantified, which disclosed an in- 
crease in their total number under the influence of exercise in the complex-running-wheel paradigm compared to inactive mice. These results are consistent with the results in CRW using CAG-CreERT2-ODD::R26R-tdTomato mice and indicate that exercise and learning activities create short windows of oxygen and metabolite starvation in active neurons due to exercise. This leads to HIF stabilization and cre activation resulting in tdTomato labelling. For the first time, our study builds on the idea of hypoxia as a normal physiological state in the brain, which is imperative for exercise and learning.

\section{Light-sheet microscopy:}

Furthermore, we explored the overall distribution of hypoxic neurons in CaMKIlaCreERT2-ODD::R26R-tdTomato transgenic mice by LSM. In the current study, mice grouped into hypoxic, normoxic, CRW and vehicle treated controls were included. Results show a global distribution of hypoxic neurons expressing CaMKIla in the whole mouse brain. Hypoxic neurons were visualized in one and 3-dimensional planes.

In summary, we report a novel and robust non-intrusive oxygen-sensing mouse model allowing the visualization of hypoxic cells under normal physiological conditions. Alterations in cellular homeostasis, for example reduced oxygen availability, are elegantly detected by CaMKIla-CreERT2-ODD::R26R-tdTomato mice. By the exposure to exercise and learning paradigms, we could show activity-induced short episodes of hypoxia in the brain, confirmed by quantification in the hippocampi of CRW mice. In conclusion, this model is suitable for studying the effects of hypoxia under normal physiological and pathological conditions, especially in neuron-targeted studies in the brain. 


\section{Original publication}

Butt, U.J., Hassouna, I., Fernandez Garcia-Agudo, L., Steixner-Kumar, A.A., Depp, C., Barnkothe, N., Zillmann, M.R., Ronnenberg, A., Bonet, V., Goebbels, S., Nave, K.A., and Ehrenreich, H. CaMKIla Expressing Neurons to Report Activity-Related Endogenous Hypoxia upon Motor-Cognitive Challenge. International journal of molecular sciences, 2021, 22(6), p.3164.

\section{Personal contributions}

I contributed to the planning and addressing the research question with my supervisor Prof. Dr. Dr. Hannelore Ehrenreich. I established the new neuron-specific hypoxia sensitive expression plasmid and further developed the transgenic mice expressing the CaMKIla-CreERT2-ODD::R26R-tdTomato mice. Furthermore, I performed the basic characterization and validation of the transgenic mice and carried out the in vivo experiments followed by quantification and analysis. I performed the hypoxia, CRW and normoxia experiments for LSM and provided the samples to our collaborator CD for processing and image acquisition. I contributed to drafting the manuscript, preparation of figures, materials and methods together with my supervisor. 


\title{
5.2 Chapter III - Original publication
}

\section{CaMKII $\alpha$ Expressing Neurons to Report Activity-Related Endogenous Hypoxia upon Motor-Cognitive Challenge}

\author{
Umer Javed Butt ${ }^{1}$, Imam Hassouna ${ }^{1}$, Laura Fernandez Garcia-Agudo ${ }^{1}$, Agnes A. Steixner-Kumar ${ }^{1}$ (D), \\ Constanze Depp ${ }^{2}$, Nadine Barnkothe ${ }^{1}$, Matthias R. Zillmann ${ }^{1} \mathbb{D}$, Anja Ronnenberg ${ }^{1}$, Viktoria Bonet ${ }^{1}$, \\ Sandra Goebbels ${ }^{2}$, Klaus-Armin Nave ${ }^{2}$ and Hannelore Ehrenreich ${ }^{1, *(1)}$ \\ 1 Clinical Neuroscience, Max Planck Institute of Experimental Medicine, 37075 Göttingen, Germany; \\ butt@em.mpg.de (U.J.B.); hassouna@em.mpg.de (I.H.); agudo@em.mpg.de (L.F.G.-A.); \\ steixner@em.mpg.de (A.A.S.-K.); barnkothe@em.mpg.de (N.B.); zillmann@em.mpg.de (M.R.Z.) \\ ronnenberg@em.mpg.de (A.R.); bonet@em.mpg.de (V.B.) \\ 2 Department of Neurogenetics, Max Planck Institute of Experimental Medicine, 37075 Göttingen, Germany; \\ depp@em.mpg.de (C.D.); sgoebbels@em.mpg.de (S.G.); nave@em.mpg.de (K.-A.N.) \\ * Correspondence: ehrenreich@em.mpg.de; Tel.: +49-551-389-9628; Fax: +49-551-389-9670
}

\section{check for
updates}

Citation: Butt, U.J.; Hassouna, L; Fernandez Garcia-Agudo, L Steixner-Kumar, A.A.; Depp, C. Barnkothe, N.; Zillmann, M.R Ronnenberg, A.; Bonet, V.; Goebbels, S.; et al. CaMKII $\alpha$ Expressing Neurons to Report Activity-Related Endogenous Hypoxia upon Motor-Cognitive Challenge. Int. J. Mol. Sci. 2021, 22, 3164 https://doi.org/10.3390/ijms22063164

Academic Editor: Luca Bonfanti

Received: 18 February 2021 Accepted: 16 March 2021 Published: 20 March 2021

Publisher's Note: MDPI stays neutra with regard to jurisdictional claims i published maps and institutional affiliations.

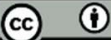

Copyright: 2021 by the author Licensee MDPI, Basel, Switzerland. This article is an open access article distributed under the terms and conditions of the Creative Commons Attribution (CC BY) license (https:// creativecommons.org/licenses/by/ $4.0 /)$
Abstract: We previously introduced the brain erythropoietin (EPO) circle as a model to explain the adaptive 'brain hardware upgrade' and enhanced performance. In this fundamental circle, brain cells, challenged by motor-cognitive tasks, experience functional hypoxia, triggering the expression of EPO among other genes. We attested hypoxic cells by a transgenic reporter approach under the ubiquitous CAG promoter, with Hif- $1 \alpha$ oxygen-dependent degradation-domain (ODD) fused to CreERT2 recombinase. To specifically focus on the functional hypoxia of excitatory pyramidal neurons, here we generated CaMKII $\alpha$-CreERT2-ODD::R26R-tdTomato mice. Behavioral challenges, light-sheet microscopy, immunohistochemistry, single-cell mRNA-seq, and neuronal cultures under normoxia or hypoxia served to portray these mice. Upon complex running wheel performance as the motorcognitive task, a distinct increase in functional hypoxic neurons was assessed immunohistochemically and confirmed three-dimensionally. In contrast, fear conditioning as hippocampal stimulus was likely too short-lived to provoke neuronal hypoxia. Transcriptome data of hippocampus under normoxia versus inspiratory hypoxia revealed increases in CA1 CaMKII $\alpha$-neurons with an immature signature, characterized by the expression of Dcx, Tbr1, CaMKII $\alpha, T l e 4$, and Zbtb20, and consistent with accelerated differentiation. The hypoxia reporter response was reproduced in vitro upon neuronal maturation. To conclude, task-associated activity triggers neuronal functional hypoxia as a local and brain-wide reaction mediating adaptive neuroplasticity. Hypoxia-induced genes such as $\mathrm{EPO}$ drive neuronal differentiation, brain maturation, and improved performance.

Keywords: physiological hypoxia; complex running wheel; hippocampus; scRNA-seq; light-shee microscopy; Hif- $1 \alpha$; neuronal differentiation; brain maturation; neuron culture; immature neurons

\section{Introduction}

Erythropoietin (EPO) is a potent hypoxia-inducible growth factor, originally named after its role in hematopoiesis, but later described to have neuroprotective functions in the nervous system [1-3]. 'Brain doping', in the sense of substantially improved maximal exercise performance without altered red blood cell production, has been suspected for a long time to be the main operative force rather than blood doping alone [4]. This was elegantly demonstrated a few years ago in a transgenic mouse line, which constitutively overexpresses human erythropoietin (EPO) exclusively in the brain [5]. However, the mechanisms explaining these dramatic erythropoiesis-independent effects and, in particular, the underlying physiology have remained widely obscure. We previously introduced the brain EPO circle as a working model explaining the adaptive 'brain hardware upgrade' and enhanced performance. In this fundamental regulatory circle, brain cells, challenged 
by motor-cognitive tasks, experience functional hypoxia, triggering the local expression of EPO, among other genes [6]. We confirmed functional hypoxic cells by a transgenic reporter approach under the ubiquitous $\mathrm{CAG}$ promoter, with the Hif- $1 \alpha$ oxygen-dependent degradation-domain (ODD) fused to CreERT2-recombinase [7].

The brain EPO circle matches perfectly with well-established, but mechanistically unexplored, experimental and clinical observations that physical activity and cognitive challenge induce comprehensive brain activation, thereby improving global brain function and increasing brain dimensions [8-10]. We showed that physiological, endogenous hypoxia is likely a respective lead mechanism, regulating neuroplasticity via assimilated gene expression in neurons of the behaving brain [7]. Importantly, targeted deletion of the EPO receptor from pyramidal neurons eliminated the observed improvement in performance [6].

These observations placed pyramidal neurons in the center of our interest and stimulated us to generate a new mouse line using a pyramidal neuronal promoter. The CaMKII $\alpha$ CreERT2-ODD::R26R-tdTomato mice presented here allowed us to specifically concentrate on the functional hypoxia of the excitatory pyramidal neurons using immunohistochemistry including light-sheet microscopy (LSM) for three-dimensional views. Additionally, hippocampal single-cell mRNA-seq transcriptome data under normoxia versus inspiratory hypoxia, focusing on CaMKII $\alpha$ expressing neurons, revealed the first hints of accelerated neurodifferentiation, pointing to enhanced neuroplasticity and brain maturation upon hypoxia.

\section{Results and Discussion}

2.1. Generation of a New Mouse Line to Report Pyramidal Neuronal Hypoxia

In our previous work [7], we used a transgenic mouse line, driven by the universal CAG promoter (CAG-CreERT2-ODD::R26R-tdTomato, slightly modified from [11]).This reporter line allowed permanent labelling of all cells undergoing transient hypoxia by the expression of a fusion protein, comprised of Hif- $1 \alpha$ ODD and tamoxifen-inducible CreERT2 Immunohistochemical analyses identified mainly neurons as reporter-labeled cells, but also certain (much lower) percentages of astrocytes, oligodendrocytes, endothelial cells, and pericytes. Hence, to specifically focus on functional hypoxia of activity-challenged pyramidal neurons, we created CaMKII $\alpha$-CreERT2-ODD::R26R-tdTomato mice (Figure 1a)

To characterize these mice, we started out with our previous 'gold standard' of positive control derived from our experience with the CAG-line [7]. We compared mice exposed to intermittent inspiratory hypoxia $\left(6 \% \mathrm{O}_{2}\right.$ for $6 \mathrm{~h}$ daily, applied over five days) with mice under inspiratory normoxia $\left(21 \% \mathrm{O}_{2}\right)$ that were handled identically, including five tamoxifen injections as indicated (Figure $1 \mathrm{~b}$ ). This regimen led to very strong staining of the pyramidal neurons already under normoxia and an extreme labelling under hypoxia, as illustrated in Figure 1c. Using corn oil injections alone as a solvent control ('no tamoxifen') to assess the non-induced expression of the transgene ('leakiness' of the tdTomato reporter) led to hardly any labelled cells (Figure 1c; Supplementary Materials-LSM Videos S1 and S2). 


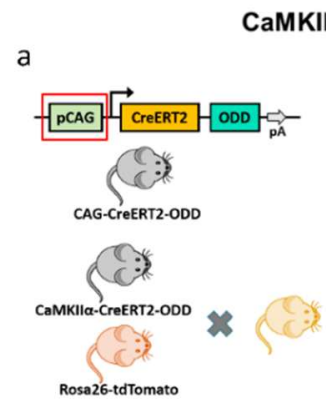

CaMKIla-CreERT2-ODD::R26R-tdTomato mouse model

C

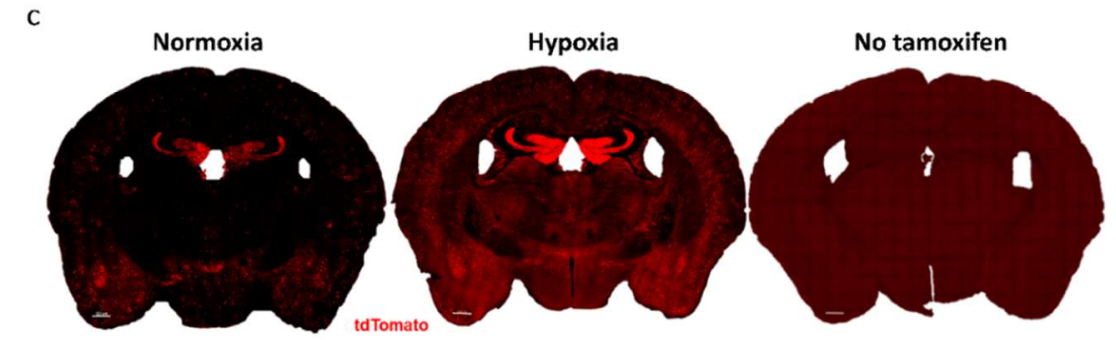

d

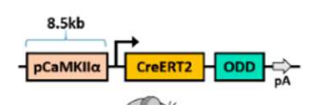

b

Design of initial screening

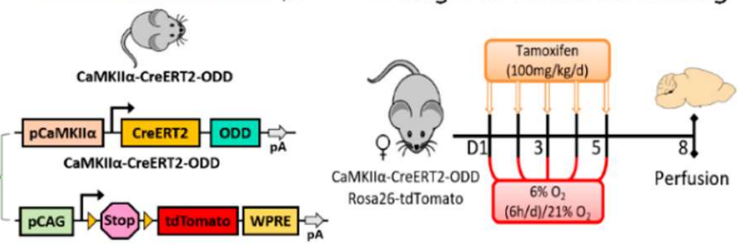

Rosa26-tdTomato

Hypoxia/tamoxifen dose-response curve

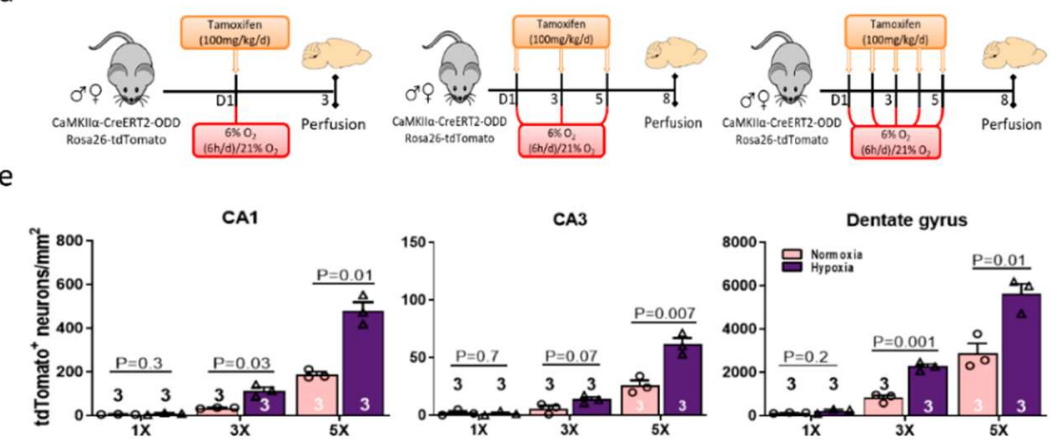

Figure 1. Generation and first characterization of excitatory neuron-specific CaMKII $\alpha$-CreERT2-ODD::R26R-tdTomato hypoxia reporter mice. (a) Schematic representation of the CAG-CreERT2-ODD construct where the CAG promoter was excised using SpeI and EcoRI restriction enzymes to obtain the vector backbone (CreERT2-ODD-pA). Subsequently, the excitatory neuronal promotor CaMKII $\alpha$ of $8.5 \mathrm{~kb}$ was inserted into CreERT2-ODD-pA to generate the CaMKIII-CreERT2ODD-pA vector. Next, the linearized vector was used for the generation of CaMKII $\alpha$-CreERT2-ODD expressing transgenic mice. These were crossed with CAG-Rosa26-tdTomato reporter mice. Permanent labelling of hypoxic neurons is achieved via Hif-1 $\alpha$ oxygen-dependent degradation domain (ODD) stabilization upon tamoxifen injection. (b) Experimental design: For an initial screening, CaMKII $\alpha$-CreERT2-ODD::R26R-tdTomato hypoxia reporter mice received tamoxifen five times over five days, each time followed by exogenous hypoxia $\left(6 \% \mathrm{O}_{2}\right.$ for $\left.6 \mathrm{~h}\right)$ versus normoxia $\left(21 \% \mathrm{O}_{2}\right)$, and mice were sacrificed on day eight. (c) Representative coronal images of five-times tamoxifen-injected CaMKII $\alpha$-CreERT2-ODD::R26RtdTomato mice under normoxic or hypoxic conditions. The corn oil control ('no tamoxifen') shows only very few tdTomato+ (red) neurons, i.e., minimal tamoxifen-independent Cre-activity. Scale bar represents $500 \mu \mathrm{m}$. (d) Experimental scheme of the hypoxia/tamoxifen dose response curve in CaMKII $\alpha$-CreERT2-ODD::R26R-tdTomato mice. (e) Quantification of tdTomato+Ctip2+ double-positive neurons in CA1, CA3 and dentate gyrus from eight-week-old mice of equally distributed mixed gender. Unpaired Student's $t$-test (two-tailed, Welch's corrections) was used for statistical analysis between groups; $\mathrm{n}$ numbers given in graphs; error bars indicate standard error of mean (SEM). 


\subsection{Critical Considerations Regarding Our Model and Proof-of-Concept Testing}

The pCaMKII $\alpha$-CreERT2-ODD transgene encodes a Cre-fusion protein that is made and immediately inactivated by two independent posttranslational mechanisms, which are both active: (1) The estrogen receptor (ER) domain is chaperoned by heat shock protein $\mathrm{Hsp} 90$ and thus the entire fusion protein is maintained inactive in the cytoplasm: Here, no Cre recombination is possible; (2) The ODD domain causes the hydroxylation of the entire fusion protein (by PHD/prolyl-hydroxylase domain proteins, followed by polyubiquitination via the VHL proteins/Von Hippel-Lindau tumor suppressor, leading to proteasomal degradation). Also here, no Cre recombination is possible. Cre recombination only occurs when both inhibitory mechanisms are blocked at the same time, i.e., by the coincidence of tamoxifen (binding to the ER domain and releasing Hsp90) and hypoxia (inactivating PHD and thereby stabilizing CreERT2-ODD). Nuclear Cre activity, even if short and only at a low level, causes the permanent activation of our Rosa26-tdTomato reporter gene by removing a STOP sequence from the DNA upstream of its coding sequence (Figure 1a), rendering the RNA transcript translatable. Consequently, all Cre-recombined cells become stably red labelled (independent of further tamoxifen and/or hypoxia). Thus, we achieved permanent staining of once hypoxic cells.

However, in order to work, a sufficient concentration of Hsp90 as well as of PHD/VHL proteins to match the cellular abundance of CreERT2-ODD are needed. Too much CreERT2ODD could out-titrate either Hsp90 and/or PHD+VHL proteins and thus result in unspecific Cre activity ('leakiness' of the system). Reassuringly, this is very low in our system as described above (Figure 1c; Supplementary Materials-LSM Videos S1 and S2).

On the other hand, the abundance of neuronally expressed CreERT2-ODD may be critical for the PHD/VHL system to work. When expressed under the control of strong promoters (both, pCAG used in [7], and pCaMKII $\alpha$ are strong promoters), the ODD domain may out-titrate the abundance of PHD and/or VHL proteins, whose expression level is regulated to exactly match the endogenous Hif proteins. These operate at a much lower abundance level, are not 'inducible' as Hsp90 proteins are, and PHD/VHL out-titration should not cause cellular stress. Therefore, we checked by qPCR whether known target genes of Hif- $1 \alpha$ are upregulated under normoxia and without any hypoxia stimulation, simply due to a high-level expression of the ODD domain in transgenic mice. Again reassuringly, the comparative qPCR results for the selected Hif-regulated genes Vegfa, Higdf1a, and Epo, obtained under normoxic conditions from the hippocampal tissue of pCaMKII $\alpha$-CreERT2-ODD and pCAG-CreERT2-ODD versus wildtype mice of the same background strain, age, and gender, did not show differences (data not shown).

\subsection{Evaluation of a Hypoxia/Tamoxifen Dose-Response Curve}

Encouraged by the outcome of these necessary controls, we next performed comparative quantifications of tdTomato+ neurons upon tamoxifen injection immediately before hypoxia, applied either once, thrice, or five times (each $6 \% \mathrm{O}_{2}$ for $6 \mathrm{~h}$ ), or analogously before normoxia, in cornu ammonis hippocampi (CA1 and CA3), as well as in the dentate gyrus. The quantification results made us select the three-times tamoxifen/hypoxia condition as a suitable titration result, i.e., as the appropriate 'gold standard' of tamoxifen injections to be applied in this particular mouse line (Figure 1d,e).

\subsection{Immunohistochemical Characterization of Hypoxic Neurons in Brain and Primary Hippocampal Neuronal Cultures}

Figure 2 provides illustrating immunohistochemical images of functional hypoxia, giving examples of tdTomato-stained excitatory neurons in the hippocampus and its subfields, various cortical areas and striatum/corpus callosum. We note the strong tdTomato labelling of hypoxic pyramidal neuronal cell bodies and the abundant neuropil staining in the hippocampus. In particular, mossy fibers in the vicinity of CA3 (stratum lucidum) and granule cell projections in the dentate gyrus are strongly labelled. The whole dentate gyrus reveals densely packed hypoxic neurons with intense fiber staining. 


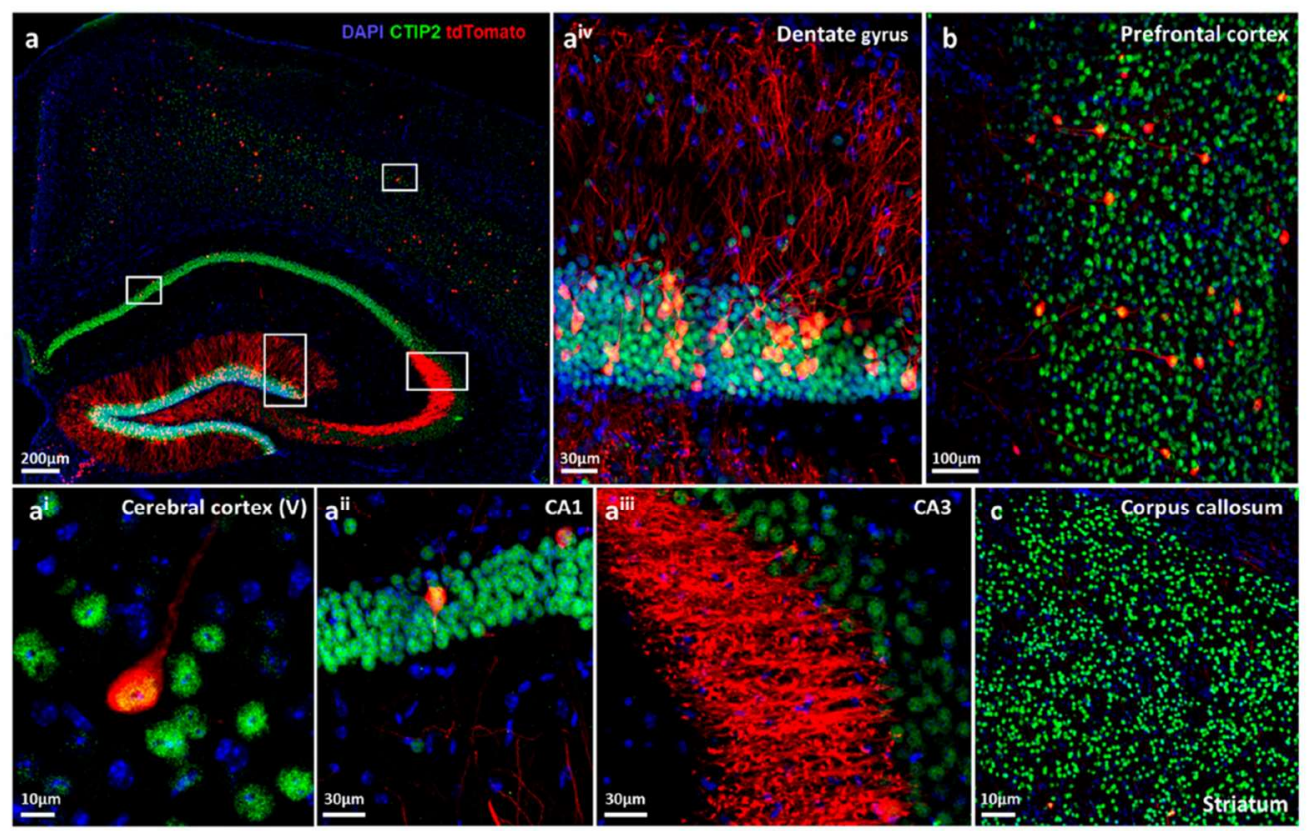

Figure 2. Immunohistochemical characterization of functional hypoxic pyramidal neurons in CaMKII $\alpha$-CreERT2ODD::R26R-tdTomato reporter mice. (a) Overview image of an eight-week-old female mouse after exposure to complex running wheel (CRW) showing distinct labelling of neurons with tdTomato fluorescent protein (red), co-immunostained with the neuronal marker Ctip2 (green) and DAPI (blue) as nuclear counterstain. White frames denote magnifications

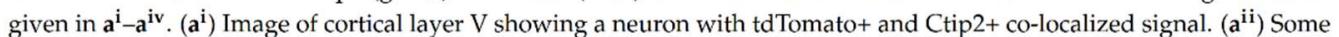
cornu ammonis (CA1) pyramidal neurons are tdTomato positive. $\left(\mathbf{a}^{\mathrm{iii}}\right)$ In CA3, just a few neuronal cell bodies display the tdTomato signal; however, strong staining is observed in stratum lucidum, rendering neuronal morphological details such as dendritic spines and synapses easily distinguishable at high resolution. $\left(\mathrm{a}^{\mathrm{iv}}\right)$ Close-up image of the superior plate of the dentate gyrus, demonstrating densely tdTomato-expressing granular neurons and remarkable labelling of neutropil. (b) A subset of neurons in prefrontal cortex and their processes are also tdTomato labelled. (c) CaMKII $\alpha$ expressing tdTomato+ neurons are sparsely present in the striatum. Scale bars represent $200 \mu \mathrm{m}(\mathbf{a}), 10 \mu \mathrm{m}\left(\mathbf{a}^{\mathbf{i}}, \mathbf{c}\right), 30 \mu \mathrm{m}\left(\mathbf{a}^{\mathrm{ii}}, \mathbf{a}^{\mathrm{iii}}\right.$ and $\left.\mathbf{a}^{\mathrm{iv}}\right)$, and $100 \mu \mathrm{m}$ (b).

In primary E17 hippocampal neuronal cultures, immediate addition of (Z)-4hydroxytamoxifen $(2 \mu \mathrm{M})$ from day in vitro (DIV) 0 onwards, under continuous hypoxia $\left(1 \% \mathrm{O}_{2}\right)$ versus normoxia $\left(21 \% \mathrm{O}_{2}\right)$, revealed tdTomato labelling on DIV16, clearly enhanced under hypoxia. In contrast, no staining was seen on DIV7 (data not shown), indicating that some degree of neuronal maturation is a prerequisite for the activity of the CaMKII $\alpha$ promoter, and thus for the expression of the CreERT2-ODD fusion protein, leading to permanent labelling of hypoxic cells (Figure 3a-d). 
a CaMKIIla-CreERT2-

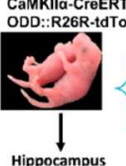

CaMKIla-CreERT2-ODD::R26R-tdTomato hippocampal culture

-

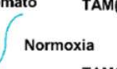

TAM $(2 \mu \mathrm{M}) / \mathrm{EtOH}$

isolation

Seeding DIV

$\left.\right|_{\text {DIv } 0}$

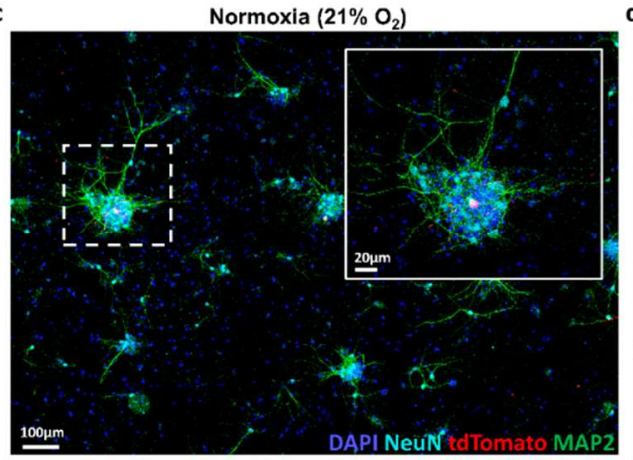

d b

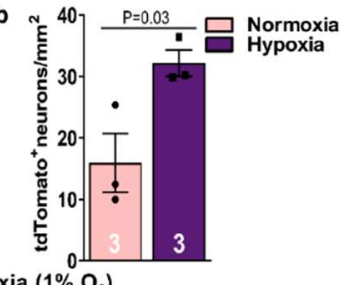

Hypoxia $\left(1 \% \mathrm{O}_{2}\right)$

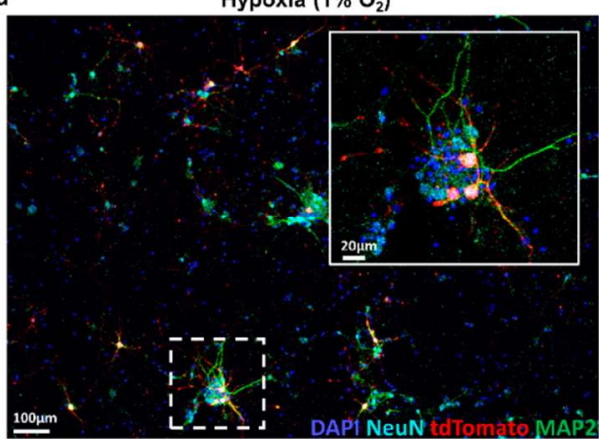

Figure 3. Maturing hippocampal neurons from CaMKII $\alpha$-CreERT2-ODD::R26R-tdTomato mice respond to hypoxia also in vitro. (a) Experimental design: Isolation and culture of hippocampal neurons from E17 pups. (Z)-4-hydroxytamoxifen $(2 \mu \mathrm{M})$ or solvent control (final EtOH concentration always $<0.016 \%$ ) are added to neuron cultures on day in vitro (DIV) 0 , followed by incubation in the IncuCyte ${ }^{\mathrm{R}}$ under either normoxia $\left(21 \% \mathrm{O}_{2}\right)$ or hypoxia $\left(1 \% \mathrm{O}_{2}\right)$ for seven or 16 days, fixation, staining, and quantification. (b) Quantification of hypoxic neurons, as visualized by tdTomato+ label, reveals an increase under hypoxia at DIV16. (c,d) Representative images of hippocampal neurons stained with neuronal markers NeuN (light blue), MAP2 (green) demonstrate co-localization with tdTomato (red), as shown strongly in hypoxic and less prominent in normoxic conditions. DAPI (blue) was used as a nuclear counterstain. Scale bars represent $100 \mu \mathrm{m}$ in overview and $20 \mu \mathrm{m}$ in magnified images. Unpaired Student's $t$-test (one-tailed, Welch's corrections) was used for statistical analysis between conditions; $n$ numbers given in graphs; error bars indicate standard error of mean (SEM).

2.5. A Challenging Complex Motor-Cognitive Task but Not a Brief Conditioning Stimulus Induces Functional Neuronal Hypoxia in the Hippocampus

Using complex running wheel (CRW) performance as a challenging motor-cognitive task, a distinct increase in functional hypoxic neurons was assessed immunohistochemically in eight-week-old male and female mice (Figure $4 a-c)$. Particularly in the dentate gyrus, tdTomato labeled neurons were highly abundant under functional hypoxia. Similar to what we observed upon inspiratory hypoxia quantifications (Figure 1e), they were rarer in CA1 and much rarer in CA3. As reported and extensively discussed earlier with the CAGCreERT2-ODD::R26R-tdTomato mice [7], females displayed distinctly stronger labelling as compared to males (overall gender comparison $p$ values CA1: $7.5 \times 10^{-5}$, CA3: 0.0034 and dentate gyrus: 0.00035; unpaired Welch's $t$-test, two-tailed). 
a
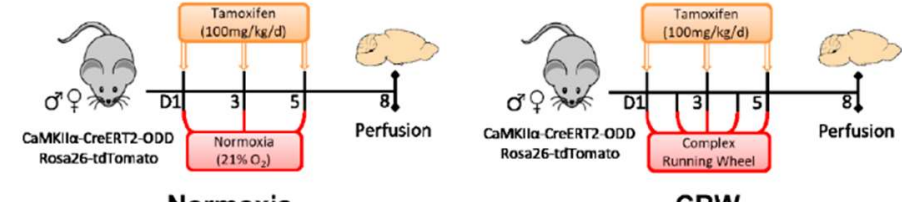

b

Normoxia

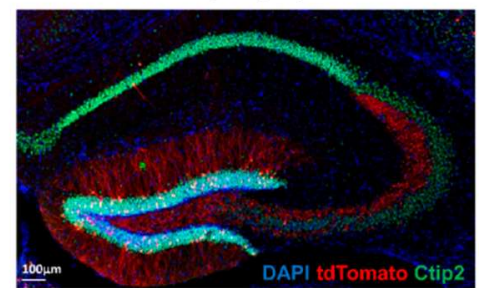

CRW

CA1

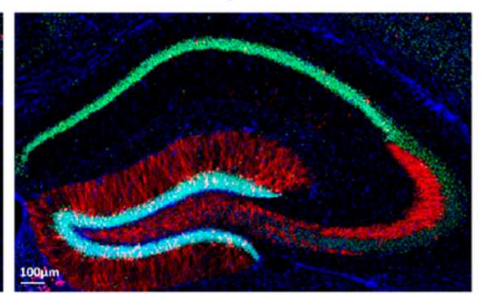

CA3
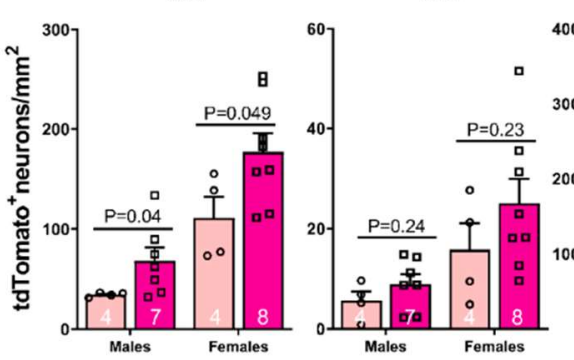

Dentate gyrus

Fear conditioning does not induce 'functional hypoxia'

d

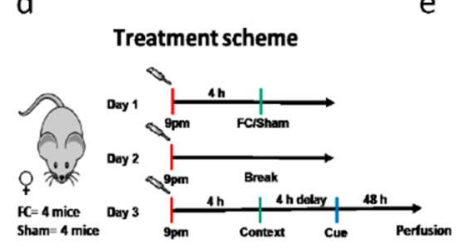

e 므 Sham CA1

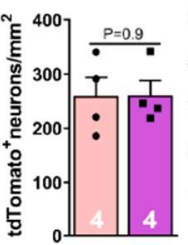

CA3 Dentate gyrus

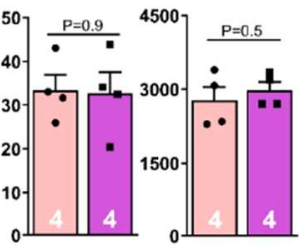

Figure 4. Complex motor-cognitive challenge as inducer of 'functional hypoxia' in the hippocampus. (a) Experimental design: mice with no prior training were given free access to complex running wheels (CRW) for five complete days and received tamoxifen injections every other day (three in total). Normoxia mice obtained the same treatment but were kept in standard cages. All mice were perfused on day eight and brains collected for histology. (b) Representative hippocampus images from eight-week-old female CRW-exposed and normoxia mice; scale bar $100 \mu \mathrm{m}$. (c) Quantifications of tdTomato+/Ctip2+ neurons in CA1, CA3, and dentate gyrus revealed an increase upon exposure to CRW in both genders, with normoxia and CRW values considerably more pronounced in females. (d) Experimental design of the fear conditioning (FC) pilot experiment (24-week-old females). (e) Quantification of tdT+/Ctip2+ neurons in hippocampal CA1, CA3, and dentate gyrus regions of sham and FC mice indicate no noteworthy difference. This is likely due to the FC stimulus being too short-lived to evoke functional hypoxia. Unpaired Student's $t$-test (two-tailed, Welch's corrections); $\mathrm{n}$ numbers given in graphs; error bars indicate standard error of mean (SEM).

We next wondered whether a very brief conditioning stimulus, leading to an extended imprinting of a negative memory (in this case, foot shocks in a defined context, also combined with a tone), would equally result in functional hypoxia of the involved hippocampal neurons. In contrast to CRW, however, contextual fear conditioning as hippocampal 
stimulus combined with cue memory as primarily amygdala readout was likely too shortlived and 'weak' to provoke detectable neuronal hypoxia in a pilot group of female mice (Figure $4 d, e)$.

Lightsheet microscopy allowed a three-dimensional presentation of stained neurons, with fly-through stacks yielding an ostensive dose-response curve from the nearly negative 'no tamoxifen' condition (lack of substantial non-induced expression of the transgene) to normoxia and to hypoxia (Supplementary Materials-LSM Video S1). In fact, CRW-induced functional neuronal hypoxia was so strong that the tdTomato labeling reached the overall level obtained by inspiratory hypoxia, offering to pool and analyze these two hypoxia conditions together (Figure 5a-c). LSM quantifications under hypoxia (both functional and inspiratory) compared to normoxia (sitting controls) showed a clear trend towards an increase but failed statistical significance due to the considerable mouse-to-mouse variation resulting in a relatively large scatter (Figure 5c; Supplementary Materials-LSM Video S2).

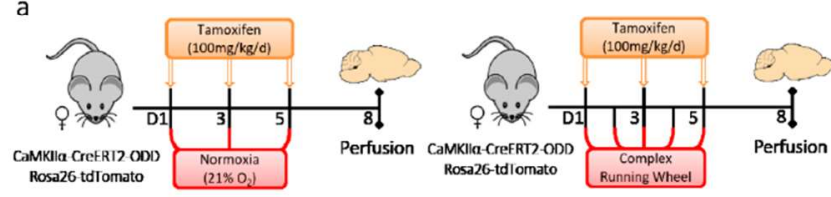

b
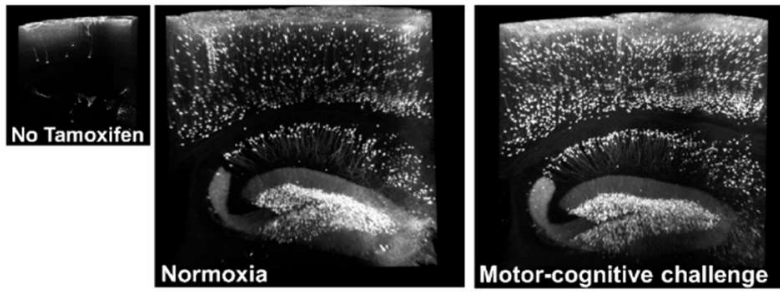

C

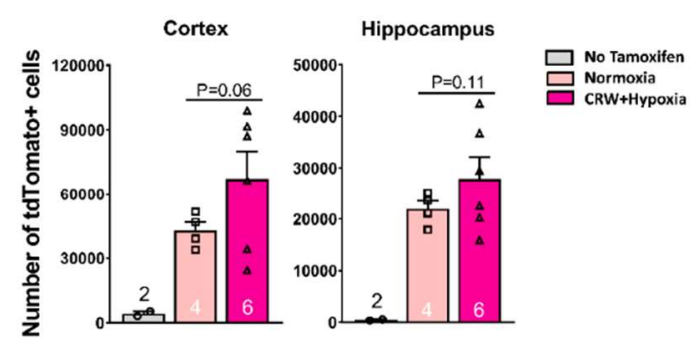

Figure 5. Light-sheet microscopy (LSM) enables 3D presentation of hypoxic neurons in CaMKII $\alpha$ CreERT2-ODD::R26R reporter mice. (a) Experimental design. (b) Illustrative 3D images rendered in maximal intensity modus demonstrate tdTomato+ hypoxic neurons in hippocampus and cortex of female normoxia versus CRW mice; the small image on the left displays a 'no tamoxifen' control brain with very few scattered tdTomato+ neurons. Scale: $1.96 \times 2.28 \times 0.4 \mathrm{~mm}$. (c) LSM quantification of tdTomato+ cells in cortex and hippocampus of eight-week-old female mice show a clear tendency of an equally strong increase under CRW and hypoxia (groups thus pooled) as compared to normoxia, with the 'no tamoxifen' condition being negligible. Unpaired Student's $t$-test (two-tailed, Welch's corrections); n numbers given in graphs; error bars indicate standard error of mean (SEM). 
2.6. Transcriptome Data Reveal Profound Regulation of CamkII $\alpha$ Expressing Neurons Upon Hypoxia vs. Normoxia

We next employed our scRNA-seq dataset of mouse hippocampus (from CAG-CreERT2ODD::R26R-tdTomato mice) collected upon normoxia $\left(21 \% \mathrm{O}_{2}\right)$ versus inspiratory hypoxia (6\% $\mathrm{O}_{2}$ for six hours/day) for five consecutive days (compare Figure 1b; GSE162079 [7]), to evaluate the hypoxia regulation of all cells expressing the CaMKII $\alpha$-promoter. In the first step, we selected all glutamatergic neuronal populations (including mossy fiber cells), known to express CaMKII $\alpha$ [12-15] from the hippocampal dataset and compared the expression levels of CaMKII $\alpha$ under normoxic and hypoxic conditions (Figure 6a). Surprisingly, CaMKII $\alpha$ was consistently upregulated under hypoxia in the excitatory neurons from the three discernible hippocampal regions (CA1, CA3, dentate gyrus). Given that CaMKII $\alpha$ is known to be a crucial mediator of synaptic plasticity, longterm potentiation as well as memory formation and learning [16-19], this hypoxia-dependent regulation might point to a common pathway that is activated via endogenous functional hypoxia upon neuronal activation and mirrored by exogenous inspiratory hypoxia. In fact, it could very well support our working model of functional hypoxia-induced 'brain hardware upgrade'.

Importantly, neither the overall expression level of CaMKII $\alpha$ nor the strength of upregulation under hypoxia in the three hippocampal regions were indicative of the amount of tdTomato-labelling observed in immunohistochemistry; e.g., the expression of CaMKII and its upregulation under hypoxia was strongest in CA3 (CaMKII $\alpha$ expression CA3 versus CA1: $p=1.68 \times 10^{-52}, \operatorname{logFC}=0.25$; CA3 versus the dentate gyrus: $p=9.96 \times 10^{-9}$, $\log \mathrm{FC}=0.16$ ), whereas the number of tdTomato-labelled neurons (IHC) was lower in $\mathrm{CA} 3$ when compared to CA1 and the dentate gyrus (Figures 1e and 4c). Overall, baseline expression of CaMKII $\alpha$ was high, with $97 \%, 99 \%$, and $93 \%$ of excitatory neurons expressing this gene under normoxia in CA1, CA3, and the dentate gyrus, respectively. Thus, although a certain amount of hypoxia 'over-reporting' - driven by the increased activity of the promoter under hypoxia-cannot be entirely excluded, the regional differences in the strength of hypoxic response we observed with immunohistochemical quantification (tdTomato-labelling) likely reflect true differences in the physiological response of neurons from different hippocampal regions to hypoxia. A systematic error affecting regional differences therefore seems highly unlikely.

In order to further explore the transcriptomic hypoxia response of CaMKII $\alpha$-neurons, we performed differential expression analyses. Figure $6 \mathrm{~b}$ depicts the top 20 up- and top 20 downregulated genes under hypoxia after clustering cells and genes by k-means clustering. The major regulated gene clusters included genes of the tubulin (cluster 2) and hemoglobin (cluster 5) family as well as ribosomal genes (cluster 4). Clustering of cells revealed three distinct cell populations with cluster 2 and 3 almost exclusively corresponding to either cells derived from mice exposed to normoxia (cluster 2 ) or hypoxia (cluster 3 ). In contrast, cluster 1 was composed of cells from both conditions showing a somewhat 'intermediate' expression of hypoxia-regulated genes, e.g., see gene clusters 2,4 , and 5 . The subpopulation of cells, which was derived from mice kept at normoxic conditions but appeared to have a mild hypoxia transcriptome signature, might represent cells that recently underwent physiological functional hypoxia. Notably, these cells were mainly situated in the dentate gyrus, a hippocampal region that harbors a neurogenic niche known to require hypoxia for its functionality [21]. 
a

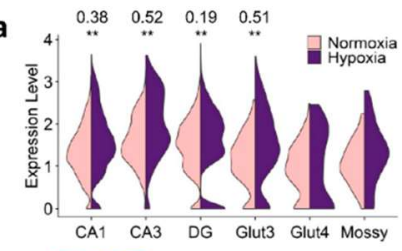

C
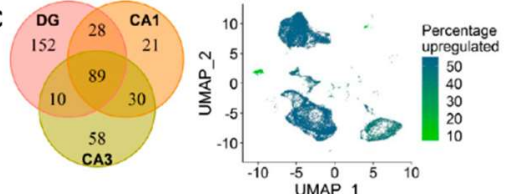

d

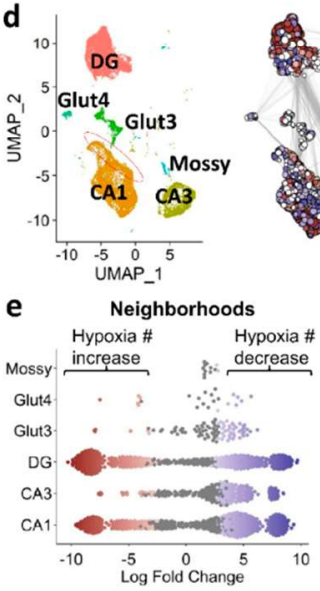

Tle4

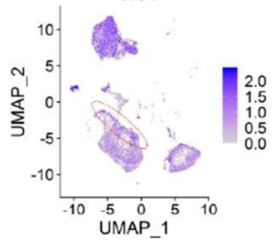

Dcx b
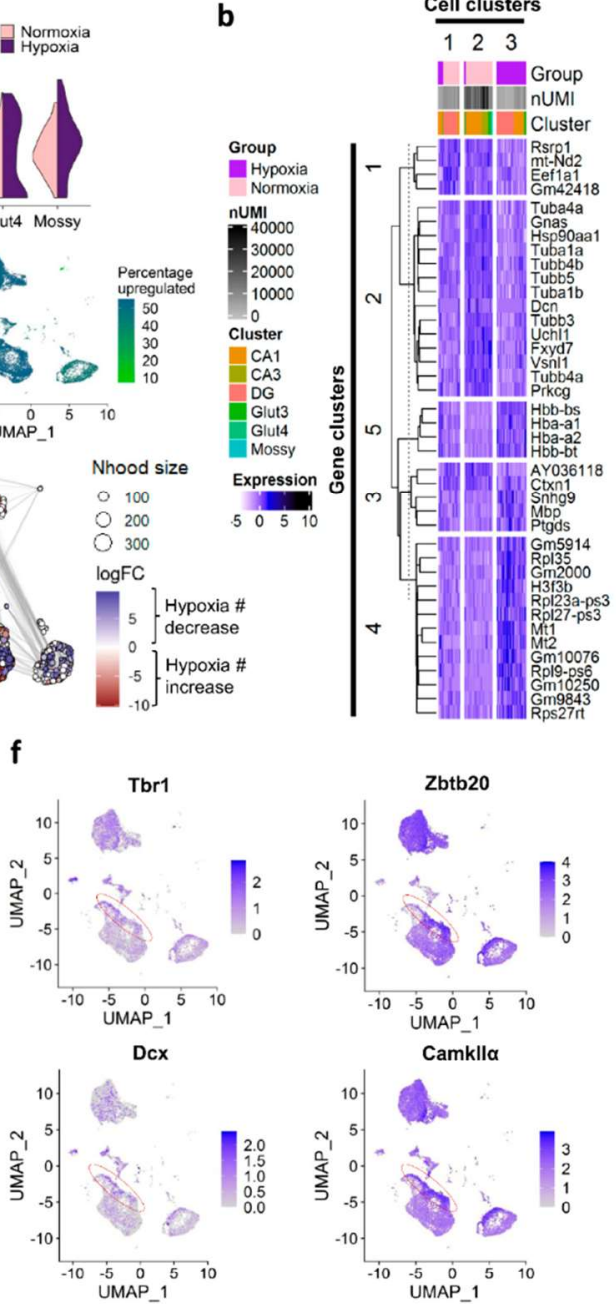
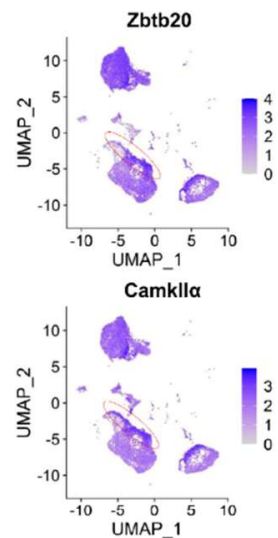

Figure 6. Response of hippocampal excitatory neurons to hypoxia evaluated by scRNA-seq. (a) Upregulation of CaMKII $\alpha$ under hypoxia (five days, $6 \% \mathrm{O}_{2}$ for $6 \mathrm{~h} /$ day) in excitatory neurons of the hippocampus. (b) Heatmap of top 20 upregulated and top 20 downregulated genes under hypoxia. Columns represent cells, rows represent genes. Scaled normalized expression shown. Cells and genes were clustered using the k-means algorithm. Number of unique molecular identifiers per cell: nUMI. (c) Left panel: Venn diagram depicting number of shared and uniquely regulated genes under hypoxia amongst excitatory neurons of different hippocampal regions (CA1, CA3, dentate gyrus). All clusters were randomly down-sampled to $n=2296$ cells for better comparability. Right panel: Percentage of upregulated genes amongst all hypoxia-regulated genes; | log-fold change I > 0.25, p-adj < 0.05, correlation-adjusted Wilcoxon test. (d) Differential cell abundance testing with MiloR [20]. Red (= negative $\log F$ ) represents a numerical increase upon hypoxia in the respective neighborhood, blue (= positive $\log \mathrm{FC}$ ) indicates a decrease. Region marked in red specifies region of immature and intermediately mature neurons of CA1 increased in number under hypoxia. $\log F C$ : $\log$-fold change, Nhood size: neighborhood size. (e) Beeswarm plot showing the numerical increases and decreases under hypoxia in the respective neighborhoods of each cell cluster. Red (=negative $\log \mathrm{FC}$ ) indicates an increase under hypoxia, blue (= positive logFC) a decrease. (f) Expression of immature and intermediately mature excitatory neuron markers. Red oval indicates again the interesting region denoted in (d) with increase in cell abundance upon hypoxia. 
In addition, the number of (uniquely) hypoxia regulated genes $(\mid \log \mathrm{FCl}>0.25$, $p_{\text {adj }}<0.05$ ) was highest in the dentate gyrus. Down-sampling of cells included into differential testing ( $n=2296$, i.e., equal to the size of CA3 as the smallest region) was performed beforehand to avoid differences in the number of detectable, differentially expressed genes due to differences in statistical power (Figure $6 \mathrm{c}$, left panel). This finding points again to a specific and elevated responsivity to hypoxia in the dentate gyrus as compared to CA. In total, 89 genes were equally regulated under hypoxia in CA1, CA3, and the dentate gyrus. As shown in Figure 6c, right panel, the percentage of upregulated genes amongst all regulated genes in $\mathrm{CA} 1$ and the dentate gyrus was $~ 54 \%$, whereas in CA3 the majority of genes was downregulated (44\% upregulated). Cluster Glut 4 , a very small cluster characterized by a high expression of immature neuronal markers such as Dcx, Tbr1, and Tle4, showed a remarkable downregulation of genes with $\sim 93 \%$ of all hypoxia differentially expressed genes being downregulated. This cluster is highly similar to the cluster of immature CA1 neurons responding to EPO already six hours after rhEPO administration that we identified earlier [6]. At this point, the physiological relevance of the strongly dampening gene regulatory response in this immature cell subpopulation remains unclear, but certainly will be investigated in future studies.

Next, we investigated numerical abundance changes in the different excitatory neuron populations of the hippocampus using MiloR [20]. This tool allows a fine-grained analysis of numerical abundance shifts under hypoxia by forming neighborhoods of similar cells within each cell cluster and assessing their numerical abundance in each treatment condition. Figure 6 d,e show the numerical shifts in each neighborhood. In CA1 and the dentate gyrus, numerically increased (red) as well as numerically decreased (blue) neighborhoods were detected under hypoxia. In contrast, CA3 neurons appeared to primarily respond to hypoxia with a numerical decrease (blue). Interestingly, in the CA1 neurons, there was a small subpopulation of cells located at the rim of the cluster (red oval in Figure $6 \mathrm{~d}$ ), which were increased in number under hypoxia. Inspection of expression markers indicated that these cells were of immature neuronal identity as shown by the high expression of $T b r 1$, Zbtb20, Tle4, and Dcx (Figure 6f). In addition, CaMKIIL expression, shown to be important for neuronal maturation in the hippocampus [22], appeared to be elevated in these cells (Figure 6f). Interestingly, previous literature inconsistently reported both negative as well as positive effects of exogenously applied hypoxia on neuronal development, differentiation, and maturation [23-29]. Our current findings indicate a positive, stimulating effect on neuronal differentiation, resulting in an increased number of young neurons in CA1. Importantly, this apparent incongruence of existing findings might not only be due to the different modes and severity of hypoxia application, but also due to a previous lack of more distinct inspections of different neuronal precursor and immature neuron populations in the hippocampus, which we provide here.

\subsection{Synopsis of Our Model: Hypoxia as a Fundamental Driving Force of Demand-Oriented} Neuroplasticity and the Consequential 'Brain Hardware Upgrade'

In an extensive line of research, we have shown over the last two decades that rhEPO treatment improves cognition and increases brain matter in humans and rodents, both healthy and diseased, but the underlying mechanisms have been unknown for a long time. Just recently, we discovered a fundamental regulatory circle, in which brain networks, challenged by cognitive tasks, drift into 'functional hypoxia' that drives neurodifferentiation and dendritic spine formation via neuronal EPO synthesis [6]. These findings highlight that EPO is not only a crucial mediator of neurogenesis during embryonic life and brain development $[30,31]$, but also pivotal for adult hippocampal neurodifferentiation and neuroplasticity on demand. The neuroprotective and anti-apoptotic effects of EPO, promoting neuronal survival, together with its neurotrophic properties, certainly contribute to the observed enhanced differentiation of local silent precursors and to their undisturbed maturation.

From the discovery of this regulatory EPO circle, driving challenge-induced brain maturation, we moved on to study the hypoxia response of all brain cells [7], and in the 
present work specifically of pyramidal neurons, employing our transgenic mouse models pCAG-CreERT2-ODD and pCaMKII $\alpha$-CreERT2-ODD, respectively.

In the sense of 'dosis facit venenum', strong oxygen deficit or hypoxia in the brain, as seen after cardiac arrest or in stroke, is definitely a serious state of emergency leading to permanent brain damage. Nevertheless, as shown here, functional hypoxia can be an important signal for growth and a driving force of the 'brain hardware upgrade'.

Hypoxia induces a specific transcriptional program, enabling cells to adapt to lower oxygen levels and/or to inadequate metabolic support. The transcription is to some degree controlled by hypoxia-inducible factors, binding to hypoxia-responsive elements to modulate the expression of many genes, some of which are potent growth factors such as vascular endothelial growth factor (VEGF) or EPO. Newer work, however, has revealed that hypoxia-induced gene transcription is partly Hif-independent [32,33]. Hence, the immunohistochemical findings reported here including LSM are based on Hif mechanisms, and may, therefore, only partly reflect the global cellular hypoxia response. In contrast, our transcriptome data seem to approach the whole hypoxia regulation more fundamentally. Overall, they strongly support the pivotal effects of hypoxia on neuronal differentiation and brain maturation.

Ultimately, moderate hypoxia, at least partly via brain EPO expression, may evolve as a highly promising add-on treatment strategy for neurodevelopmental, neuroinflammatory, and neurodegenerative brain diseases, applicable in the sense of individualized therapeutic approaches to still untreatable conditions.

\section{Materials and Methods}

\subsection{Generation of the CaMKII $\alpha$-CreERT2-ODD-pAa Vector}

The genetic strategy for the generation of the CaMKII $\alpha$-CreERT2-ODD-pA vector is illustrated in Figure 1a. Briefly, the construct consists of the murine CaMKII $\alpha$ promoter, active in excitatory neurons, which drives the expression of tamoxifen-inducible Crerecombinase (Cre-ERT2), fused to the oxygen-dependent degradation domain (ODD) of Hif- $1 \alpha$. The latter responds to varying oxygen concentrations. The pCAG-CreERT2ODD-pA plasmid was kindly provided by Dr. Hashim A Sadek (USA) [11] and slightly modified [7]. In the first step, the CAG promoter was excised by digesting CAG-CreERT2ODD-pA with SpeI and EcoRI restriction enzymes. In parallel, the CaMKII $\alpha$ promoter fragment of $8.5 \mathrm{~kb}$ was obtained by digesting the CaMKII $\alpha$-HA-cEPOR vector $[34,35]$ with XmaI, NdeI, PacI, and NotI restriction enzymes (New England Biolabs, USA). In a second step, this CaMKII $\alpha$ promoter fragment was ligated upstream into the CreERT2-ODD vector to yield CaMKII $\alpha$-Cre-ERT2-ODD-pA.

\subsection{CamkII $\alpha$-CreERT2-ODD: R26R-tdTomato Transgenic Mice: Generation \& Validation}

CaMKII $\alpha$-Cre-ERT2-ODD-pA was digested with the SfiI and SalI (New England Biolabs, USA) restriction enzymes and the ampicillin selection sequence removed. After gel elusion with the QIAquick gel extraction kit (Qiagen, Venlo, Netherlands), linearized DNA of $11.6 \mathrm{~kb}$ was obtained comprising CaMKII $\alpha$ promoter, inducible Cre-recombinase, and ODD sequence. The linearized DNA was used for pro-nuclear microinjection into fertilized eggs for the production of CaMKII $\alpha$-CreERT2-ODD transgenic mice. Litters from the foster mothers were screened for the presence of CaMKII $\alpha$ and ODD sequence by genotyping as explained below for suitable transgenic founders. CaMKII $\alpha$-CreERT2-ODD transgenic mice were maintained on C57BL/6N background (Charles River, MA, USA) and F1 litters carrying the CaMKII $\alpha$-CreERT2-ODD transgene were selected and further crossed with C57BL/6N and R26R-tdTomato mice for colony maintenance and to generate the desired heterozygous reporter mice. F2 litters of both genders harboring the CaMKII $\alpha$ CreERT2-ODD::R26R-tdTomato transgene were used for the experiments. All transgenic mice showed normal breeding, home cage behavior and life expectancy, and lacked any obvious abnormal phenotype. 


\subsection{Genotyping}

Genotyping polymerase chain reaction (PCR kit, Biozym, Oldendorf, Germany) was performed by using the following primers for CaMKII $\alpha$ promoter, forward: $5^{\prime}$-GGTTCTCCGTTT GCACTCAGGA- $3^{\prime}$ and reverse: $5^{\prime}$-CCATGAGTGAACGAACCTGG- 3 and for ODD forward: $5^{\prime}$-GCTGAAGACACAGAAGCAAA-3' and reverse: $5^{\prime}$-GTGGGTAGGAGATGGAGATG- ${ }^{\prime}$. In the subsequent generation, CaMKII $\alpha$-CreERT2-ODD::R26R-tdTomato transgenic F2 offspring, harboring the tdTomato sequence were screened by the following primers: Primer1: 5'TCAATGGGCGGGGGTCGTT3'; Primer2: 5'CTCTGCTGCCTCCTGGCTTCT3'; Primer3 $5^{\prime}$ CGAGGCGGATCACAAGCAATA $3^{\prime}$. Complete PCR protocols are available on request.

\subsection{Mouse Maintenance}

All experiments were approved by and conducted in accordance with the regulations of the local Animal Care and Use Committee (Niedersächsisches Landesamt für Verbraucherschutz und Lebensmittelsicherheit, LAVES). CaMKII $\alpha$-CreERT2-ODD::R26R-tdTomato mice were kept on the C57BL/6N genetic background, group-housed, maintained in a germ-free and temperature $\left(21 \pm 2{ }^{\circ} \mathrm{C}\right)$-controlled environment on a $12 \mathrm{~h} \mathrm{light} /$ dark schedule. Adult transgenic mice from F2 generation (seven to eight weeks old) of both genders, harboring the CaMKII $\alpha$-CreERT2-ODD::R26R-tdTomato transgene, were used in the experiments unless otherwise stated. Each mouse was single-housed for habituation before the start of the experiment. All mice (normoxia, hypoxia, CRW, 'no tamoxifen') were handled in the same way, and euthanised two days after the last tamoxifen/solvent control injection. See the experimental designs in the figures for details.

\subsection{Tamoxifen Preparation}

A stock solution of tamoxifen was prepared by mixing $300 \mathrm{mg}$ tamoxifen in $30 \mathrm{~mL}$ of corn oil (both Sigma-Aldrich, Darmstadt, Germany) by sonication at $37^{\circ} \mathrm{C}$ and stored at $4{ }^{\circ} \mathrm{C}$. To label the hypoxic cells in normoxia, CRW, or hypoxia mice, a working solution of tamoxifen $(100 \mathrm{mg} / \mathrm{kg})$ was freshly warmed up, sonicated for $20 \mathrm{~min}$ before each application, and injected intraperitoneally (i.p.) as stated in the experimental designs. The 'no tamoxifen' controls received vehicle (corn oil) only.

\subsection{Hypoxia Application}

Exogenous hypoxia was applied to CaMKII $\alpha$-CreERT2-ODD::R26R-tdTomato mice in a small hypoxia chamber $(60 \mathrm{~cm} \times 38 \mathrm{~cm} \times 20 \mathrm{~cm})$, custom-designed in cooperation with Coy Laboratory Products (Michigan, USA). The hypoxia chamber is connected to an oxygen sensor, a stage controller and a ceiling fan for constant air circulation. In the hypoxia treatment group, CaMKII $\alpha$-CreERT2-ODD::R26R-tdTomato mice received a first dose of tamoxifen $(100 \mathrm{mg} / \mathrm{kg} / \mathrm{d})$ and were then put into the hypoxia chamber. The ambient oxygen concentration $\left(20.9 \% \mathrm{O}_{2}\right)$ was dropped to $6 \% \mathrm{O}_{2}$ within $15 \mathrm{~min}$, kept for 6 hand then increased back to $20.9 \% \mathrm{O}_{2}$. In the normoxia, CRW, and 'no tamoxifen' group, mice received tamoxifen injections or corn oil, respectively, and were kept in ambient oxygen concentration $\left(20.9 \% \mathrm{O}_{2}\right)$

\subsection{Complex Running Wheel (CRW) Exposure}

CRW is a simple, sensitive, and fully computerized voluntary wheel-running task, which is frequently used to detect motor-cognitive and activity impairments in mice [36,37]. The CRW (TSE Systems, Bad Homburg, Germany) is defined by irregularly spaced bars. The locomotor activity and running performance is computer-controlled via Phenomaster software (TSE Systems, Bad Homburg, Germany). Untrained CaMKII $\alpha$-CreERT2ODD::R26R-tdTomato transgenic mice (eight-week-old; both genders) were given tamoxifen injections every other day (Figure 4a) and had continued free access to CRW for five days. On day five, mice were transferred back to standard cages for two days and later perfused. 


\subsection{Fear Conditioning}

Fear conditioning (Med Associates inc, Hertfordshire, United Kingdom) was performed in a single trial (design in Figure 4d) in which, after a $120 \mathrm{~s}$ baseline period, mice experienced two paired presentations of a $10 \mathrm{~s}, 5 \mathrm{kHz}, 85 \mathrm{~dB}$ tone (conditioned stimulus) and an electrical foot shock for $2 \mathrm{~s}$ with an intensity of $0.4 \mathrm{~mA}$ (unconditioned stimulus, Context A). The control group (SHAM) consisted of mice exposed to the context only. The contextual memory was assessed $48 \mathrm{~h}$ after training (Context A). Trained mice and control mice were observed over $120 \mathrm{~s}$ for freezing inside the conditioning chamber. After $4 \mathrm{~h}$, mice were placed in an unfamiliar, new chamber (Context B) and were, after an initial $120 \mathrm{~s}$ baseline phase, re-exposed to the tone (CS) to assess cued memory/fear over $120 \mathrm{~s}$.

\subsection{Hippocampal Neuronal Culture and Image Acquisition}

For the neuronal culture, CaMKII $\alpha$-ODD-CreERT2::tdTomato fetuses were extracted from the uterus at approximately embryonic day 17 (E17). Hippocampi were dissected from the brain and the meninges were removed before digestion with $0.05 \%$ trypsin for $10 \mathrm{~min}$ at $37^{\circ} \mathrm{C}$. Subsequent washes were performed in a neuronal culture medium containing 2\% B27 (cat. no. 17504044), 1\% GlutaMAX (cat. no. 35050087), and 1\% penicillin/streptomycin (cat. no. 15140163) in Neurobasal A (cat. no. 10888022, all from Gibco, Thermo Fisher Scientific, Darmstadt, Germany). Single-cell suspension was obtained by careful dissociation of the tissue with a P200 micropipette. Seeding was conducted using poly-D-lysine (PDL) (Sigma-Aldrich, Darmstadt, Germany)-coated glass coverslips in 24-well plates, in densities of 100,000 cells/well. The same day of the preparation, fresh $2 \mu \mathrm{M}$ (Z)-4-hydroxytamoxifen (H79049, Sigma-Aldrich, Hamburg, Germany) or an equivalent volume of $99.8 \%$ pure ethanol (EtOH) (Honeywell, North Carolina, USA) were prepared and added to the cultured wells. The final $\mathrm{EtOH}$ concentration was always $<0.016 \%$. Cells were incubated at $37^{\circ} \mathrm{C}$ in $5 \% \mathrm{CO}_{2}$, in either normoxia $\left(21 \% \mathrm{O}_{2}\right)$ or hypoxia $\left(1 \% \mathrm{O}_{2}\right)$, for seven or 16 days. Then, fixation was performed with $2 \%$ acrolein (SigmaAldrich, Darmstadt, Germany) and 3\% formaldehyde (Merck, Darmstadt, Germany) in PBS. IncuCyte ${ }^{\mathrm{R}}$ ZOOM (Sartorius, Ann Arbor, MI, USA) images $(1400 \times 1400 \mu \mathrm{m})$ were taken with a $10 \times$ objective using phase contrast and the red fluorescent channel. For the neuronal quantification of tdTomato+ cells, images were processed in IncuCyte ${ }^{\mathrm{R}} \mathrm{ZOOM}$ software with TopHat parameter (radius $=100 \mu \mathrm{m}$, threshold red calibrated unit $=0.5$ ) and edge split sensitivity $=-10$.

\subsection{Tissue Preparation and Immunohistochemistry}

For tissue harvesting and perfusion, Avertin (Tribromoethanol, Sigma-Aldrich, St Louis, MN, USA, $0.276 \mathrm{mg} / \mathrm{g}$ ) was used for deep sedation by intraperitoneal injection followed by perfusion with ice cold $0.9 \%$ saline solution and $4 \%$ formaldehyde (Merck, Darmstadt, Germany). The brains were removed, placed in $4 \%$ formaldehyde at $4{ }^{\circ} \mathrm{C}$ for $24 \mathrm{~h}$, then cryoprotected in 30\% sucrose in phosphate buffered saline (PBS) (Merck, Darmstadt, Germany) at $4{ }^{\circ} \mathrm{C}$ for $48 \mathrm{~h}$, finally covered in cryoprotectant (O.C.T.TM Tissue-Tek, Sakura, The Netherlands) and preserved at $-80^{\circ} \mathrm{C}$ until use. In preparation for immunohistochemistry (IHC), brains were cut into $30-\mu \mathrm{m}$-thick coronal sections using a cryostat (Leica, Wetzlar, Germany) and stored floating in a cryoprotective solution (25\% ethylene glycol and $25 \%$ glycerol in PBS (Sigma-Aldrich, Darmstadt, Germany) at $4{ }^{\circ} \mathrm{C}$. For IHC, five sections $(30 \mu \mathrm{m})$ from each mouse were washed and blocked for $1 \mathrm{~h}$ in $5 \%$ horse serum in PBS with $0.5 \%$ Triton X-100 (Sigma-Aldrich, Darmstadt, Germany) at room temperature (RT). Primary antibodies were diluted in 3\% horse serum (Jackson ImmunoResearch, West Grove, Pennsylvania, USA) in PBS/0.3\% Triton X-100 and sections incubated for $48 \mathrm{~h}$ at $4{ }^{\circ} \mathrm{C}$, followed by washing and incubation with the respective secondary antibodies for $2 \mathrm{~h}$ at RT. For nuclear counterstaining, $4^{\prime}$,6-diamidino-2-phenylindole (DAPI, Sigma, Missouri, United States) was used. The sections were then mounted on SuperFrostPlus slides (ThermoFisher, Darmstadt, Germany) with Aqua-Polymount (Polysciences, Inc. Warrington, PA, USA). For the direct visualization of tdTomato, sections were only counterstained 
with nuclear stain (DAPI) and investigated by confocal microscope (Leica TCS SP5-II; Mannheim, Germany). The primary antibodies applied were anti-NeuN (1: 1000, mouse; Millipore, Darmstadt, Germany), and anti-Ctip2 (1: 500, guinea pig; SYSY, Göttingen, Germany). The secondary antibodies used were Alexa488 anti-guinea pig (1: 500, Invitrogen, Darmstadt, Germany), and Alexa647 anti-mouse (1: 500; ThermoFisher, Darmstadt, Germany). For the quantification of NeuN+tdTomato+ co-labelled cells, sequential coronal sections from the dorsal part of hippocampus were taken (coordinates from Bregma: -1.34 to $-2.54 \mathrm{~mm}$ posterior). Stained sections $(30 \mu \mathrm{m})$ were imaged using Leica TCS SP5 (Mannheim, Germany), equipped with a $20 \times$ objective $(N A=0.70)$. Hypoxic neurons (Ctip2+tdTomato+) from CA1, CA3, and the dentate gyrus were counted manually by Fiji software (https:/ / imagej.net/Fiji, accessed on 18 February 2021). Representative images of bilateral hippocampi from five sections per mouse were processed/analyzed using Imaris 9.1.0 (www.bitplane.com, accessed on 18 February 2021).

\subsection{Light-Sheet Microscopy (LSM)}

Whole mount tissue staining and clearing:

To visualize the tdTomato+ cells in the entire brain, we performed LSM in combination with whole mount immune labelling and tissue clearing. Following transcardial perfusion as described above, the brains were removed, post-fixed in 4\% PFA overnight and stored in PBS. The brain hemispheres were processed for immune-labelling and tissue clearing following a slightly modified iDISCO protocol [38]. The samples were dehydrated with a methanol/PBS series $(50 \%, 80 \%, 100 \%$, and $2 \times 100 \%, 1 \mathrm{~h}$, RT) followed by overnight bleaching and permeabilization in a mixture of $5 \% \mathrm{H}_{2} \mathrm{O}_{2} / 20 \%$ dimethyl sulfoxide (DMSO) (Sigma-Aldrich, Darmstadt, Germany) in methanol at $4{ }^{\circ} \mathrm{C}$. Samples were retrieved and washed further with methanol at $4{ }^{\circ} \mathrm{C}$ for $30 \mathrm{~min}$ and $-20^{\circ} \mathrm{C}$ for $3 \mathrm{~h}$ prior to incubation in $20 \%$ DMSO in methanol at RT for $2 \mathrm{~h}$. Samples were then rehydrated using a descending methanol/PBS series ( $80 \%, 50 \%$, PBS, 1 h each, RT) and further washed with in PBS $/ 0.2 \%$ TritonX-100 for $2 \mathrm{~h}$. The samples were then incubated overnight in $0.2 \%$ TritonX-100, $20 \%$ DMSO, and $0.3 \mathrm{M}$ glycine in PBS at $37^{\circ} \mathrm{C}$ and blocked using PBS containing $6 \%$ goat serum, $10 \%$ DMSO and $0.2 \%$ Triton- $\mathrm{X} 100$ for two days at $37^{\circ} \mathrm{C}$. The samples were then washed twice in PBS containing $0.2 \%$ Tween $(\mathrm{r}) 20$ and $10 \mu \mathrm{g} / \mathrm{mL}$ heparin $(\mathrm{PTwH})$ at RT for $1 \mathrm{~h}$ and incubated with primary antibody solution (anti-RFP; Rockland \# 600401-379, Pennsylvania, USA; 1: 250 in PTwH/5\%DMSO/3\% goat serum) for 14 days at $37^{\circ} \mathrm{C}$. After several washes during the day and an additional overnight wash in $\mathrm{PTwH}$, the samples were incubated with secondary antibody solution (goat anti-rabbit Alexa555; ThermoFisher Scientific Darmstadt, Germany, \# A-21428; 1: 500 in PTwH/3\% goat serum) for seven days at $37^{\circ} \mathrm{C}$. Prior to clearing, the samples were again washed in PTwH (several solution changes during the day) followed by an additional overnight wash. Tissues were dehydrated using an ascending series of methanol/PBS $(20,40,60,80$, and $2 \times 100 \% 1 \mathrm{~h}$, RT) followed by overnight incubation in a mixture of $33 \%$ dichloromethan (DCM) and $66 \%$ methanol at RT. Samples were further delipidated by incubation in 100\% DCM for $40 \mathrm{~min}$ and transferred to pure ethyl cinnamate (Eci; Sigma Aldrich \#112372, Darmstadt, Germany) as the clearing agent. The tissues became transparent after $30 \mathrm{~min}$ in Eci and were stored at RT until imaging.

LSM and 3D analysis/visualisation

LSM was performed using a LaVision Ultramicroscope II equipped with a $2 \times$ objective, corrected dipping cap and zoom body. Samples were mounted onto the sample holder with the medial surface of the brain hemisphere facing down in order to acquire sagittal images. The holder was placed into the imaging chamber filled with Eci. The images were acquired in mosaic acquisition mode with the following specifications: $5 \mu \mathrm{m}$ sheet thickness; $20 \%$ sheet width; $2 x$ zoom; $4 \times 5$ tiling; $4 \mu \mathrm{m}$ z-step size; dual site sheet illumination; $50 \mathrm{~ms}$ camera exposure time; $1000 \mathrm{px} \times 1600 \mathrm{px}$ field of view. Red fluorescence was recorded using $561 \mathrm{~nm}$ laser excitation ( 5 to 10\%) and 585/40 emission filters. Images were loaded into Vision4D 3.0 (Arivis) and stitched using the tile sorter setup. Hippocampus and 
cortex regions of interests (ROIs) where manually annotated according to the sagittal Allen mouse brain atlas [39]. For this, hippocampus and cortex ROIs were traced manually in a few planes in 2D from which the 3D ROI was extrapolated automatically. Cortex and hippocampus annotations were then cropped with a medial cut-off of approximately $0.4 \mathrm{~mm}$ and a lateral cut-off of approximately $4.4 \mathrm{~mm}$ (corresponding to the lateral end of the hippocampal formation). Cortex ROIs spanned the dorsal parts of the cortex as defined by anatomical landmarks. Next, tdTomato+ cells per ROI were identified using the blob finder algorithm in Vision4D. Noise was removed by deleting objects with voxel sizes $<10$ from the object table. Objects were then critically reviewed, and any additional noise was manually removed from the dataset. The number of tdTomato+ cells per ROI was extracted from the object table and plotted using GraphPad (Prism). For visualisation purposes, a $1300 \times 1400 \times 100$ px field of view spanning the hippocampus and overlaying the cortex was cut out from the original datasets and visualized in the 3D maximal intensity modus. Whole-brain and hippocampal 3D videos as well as 'flythrough' stacks were rendered in Vision4D.

\subsection{2. scRNA-seq}

Single-cell RNA sequencing (scRNA-seq) data was obtained and processed as described in detail earlier [7] (GSE162079). For the purpose of the current paper, only excitatory neuron populations known to express CaMKII $\alpha$ were included in the analyses. All analyses were performed using R v4.0.0 [40], except for the MiloR package, which was run in R v4.0.3. Differentially expressed genes between hypoxia and normoxia were identified using the FindMarkers() function with default settings (two tailed correlation-adjusted Mann-Whitney U test, | log-fold change I > 0.25) in Seurat v3.1.5 [41] (please note that the empty brackets indicate the use of respective functions with its default settings). The regional assignment of neuronal clusters was performed using the following markers: Mpped1 for CA1, Mndal for CA3, and Prox1 for DG. Mossy fiber cells were characterized by the strong expression of Calb2 [42], while the Glut3 cluster was showing a high expression of Tshz2, possibly indicating subicular origin [43]. Glut4 was high in the expression of Tbr1, Dcx, and Tle4, indicating an immature cell state [6]. In order to mitigate powerrelated differences in the number of detectable differentially expressed genes, clusters were down-sampled to the smallest cluster included in the comparison, i.e., CA3 with $n=2296$ (Figure $6 c$, left panel). For easier visualization, a small number of cells located far below on the UMAP2 axis were shifted upwards. The heatmap was created with the ComplexHeatmap package [44]. The Venn diagrams were created with with VennDiagram [45]. The numerical changes under hypoxia versus normoxia were investigated using the MiloR v0.1.0 [20] package. The graph was built using buildGraph $(k=30$, $d=30$ ) and neighbourhoods were defined with the same paramaters by makeNhoods() and setting the proportion of randomly sampled graph vertices to prop $=0.2$. Distances between the nearest neighbours were calculated with calcNhoodDistance $(d=30)$. Cells per neighbourhood were quanitfied with countCells(). Differential abundance testing was performed with testNhoods(design $=\sim$ experimental_group) and the results were visualized after calling buildNhoodGraph() with plotNhoodGraphDA(). On https://github.com/AgnesSteixner/Butt_et_al_Camk2a, accessed on 18 February 2021, analysis scripts are available. Bonferroni-adjusted (Seurat) or false discovery rate-adjusted (MiloR) $p$-values were reported.

\subsection{Statistical Analysis}

Data obtained for all quantifications were analyzed by Graph Pad Prism 8 (GraphPad Software, Inc. San Diego, CA, USA). The statistical significance was calculated by using an unpaired Welch's $t$-test to compare two groups. A $p$ value $<0.05$ was considered statistically significant. All bar graphs show means and error bars represent the standard error of mean (SEM). 
Supplementary Materials: The following are available online at https://www.mdpi.com/1422-0 067/22/6/3164/s1. LSM Video S1. Fly-Through-Stacks. LSM Video S2. Hippocampal Closeup 3 conditions.

Author Contributions: Concept, design and supervision of the study: H.E.; Data acquisition/analysis/ interpretation: U.J.B., I.H., L.F.G.-A., A.A.S.-K., C.D., N.B., M.R.Z., A.R., V.B., S.G., K.-A.N. and H.E.; Drafting manuscript: H.E., together with U.J.B., L.F.G.-A. and A.A.S.-K.; Drafting display items: U.J.B., I.H., L.F.G.-A., A.A.S.-K., together with H.E. All authors have read and agreed to the published version of the manuscript.

Funding: This study was supported by the Max Planck Society, the Deutsche Forschungsgemeinschaft (DFG, German Research Foundation) TRR 274/1 2020-408885537.

Institutional Review Board Statement: All experiments were approved by and conducted in accordance with the regulations of the local Animal Care and Use Committee (Niedersächsisches Landesamt für Verbraucherschutz und Lebensmittelsicherheit, LAVES).

Informed Consent Statement: Not applicable.

Data Availability Statement: Analysis scripts for scRNA-seq analysis: https: / / github.com/AgnesSteixner / Butt_et_al_Camk2a. Raw and processed scRNA-seq data are publicly available on GEO via accession code GSE162079.

Acknowledgments: U.J.B. has received a PhD stipend from National University of Sciences and Technology (NUST), Faculty Development Program Abroad 2014/15 Pakistan. A.A.S.-K. has held a stipend of the IMPRS-GGNB PhD Program Neurosciences (DFG Grant GSC 226), Göttingen. C.D. holds a Boehringer Ingelheim Fonds PhD Fellowship. K.-A.N. is supported by Adelson Medical Research Foundation and an ERC Advanced Grant.

Conflicts of Interest: The authors declare no competing financial or other interests.

\section{References}

1. Brines, M.; Cerami, A. Emerging biological roles for erythropoietin in the nervous system. Nat. Rev. Neurosci. 2005, 6, 484-494. [CrossRef]

2. Jelkmann, W. Regulation of erythropoietin production. J. Physiol. 2011, 589, 1251-1258. [CrossRef]

3. Krantz, S.B. Erythropoietin. Blood 1991, 77, 419-434. [CrossRef]

4. Grasso, G.; Alafaci, C.; Ghezzi, P. Is erythropoietin a worthy candidate for traumatic brain injury or are we heading the wrong way? F1000Research 2016, 5, 911. [CrossRef] [PubMed]

5. Schuler, B.; Vogel, J.; Grenacher, B.; Jacobs, R.A.; Arras, M.; Gassmann, M. Acute and chronic elevation of erythropoietin in the brain improves exercise performance in mice without inducing erythropoiesis. FASEB J. 2012, 26, 3884-3890. [CrossRef] [PubMed]

6. Wakhloo, D.; Scharkowski, F.; Curto, Y.; Butt, U.J.; Bansal, V.; Steixner-Kumar, A.A.; Wüstefeld, L.; Rajput, A.; Arinrad, S.; Zillmann, M.R.; et al. Functional hypoxia drives neuroplasticity and neurogenesis via brain erythropoietin. Nat. Commun. 2020, 11, 1-12. [CrossRef] [PubMed]

7. Butt, U.J.; Steixner-Kumar, A.A.; Depp, C.; Sun, T.; Hassouna, I.; Wüstefeld, L.; Arinrad, S.; Zillmann, M.R.; Schopf, N.; Garcia-Agudo, L.F.; et al. Hippocampal neurons respond to brain activity with functional hypoxia. Mol. Psychiatry 2021 1-18. [CrossRef]

8. Erickson, K.I.; Hillman, C.; Stillman, C.M.; Ballard, R.M.; Bloodgood, B.; Conroy, D.E.; Macko, R.; Marquez, D.X.; Petruzzello, S.J.; Powell, K.E. Physical Activity, Cognition, and Brain Outcomes: A Review of the 2018 Physical Activity Guidelines. Med. Sci. Sports Exerc. 2019, 51, 1242-1251. [CrossRef] [PubMed]

9. Kramer, A.F.; Erickson, K.I. Capitalizing on cortical plasticity: Influence of physical activity on cognition and brain function. Trends Cogn. Sci. 2007, 11, 342-348. [CrossRef]

10. Pajonk, F.-G.; Wobrock, T.; Gruber, O.; Scherk, H.; Berner, D.; Kaizl, I.; Kierer, A.; Müller, S.; Oest, M.; Meyer, T.; et al. Hippocampal Plasticity in Response to Exercise in Schizophrenia. Arch. Gen. Psychiatry 2010, 67, 133-143. [CrossRef] [PubMed]

11. Kimura, W.; Xiao, F.; Canseco, D.C.; Muralidhar, S.; Thet, S.; Zhang, H.M.; Abderrahman, Y.; Chen, R.; Garcia, J.A.; Shelton, J.M.; et al. Hypoxia fate mapping identifies cycling cardiomyocytes in the adult heart. Nature 2015, 523, 226-230. [CrossRef]

12. Benson, D.; Isackson, P.; Gall, C.; Jones, E. Contrasting patterns in the localization of glutamic acid decarboxylase and Ca2+ /calmodulin protein kinase gene expression in the rat centrat nervous system. Neuroscience 1992, 46, 825-849. [CrossRef]

13. Jones, E.; Huntley, G.; Benson, D. Alpha calcium/calmodulin-dependent protein kinase II selectively expressed in a subpopulation of excitatory neurons in monkey sensory-motor cortex: Comparison with GAD-67 expression. J. Neurosci. 1994, 14, 611-629. [CrossRef] 
14. Sík, A.; Hájos, N.; Gulácsi, A.; Mody, I.; Freund, T.F. The absence of a major $\mathrm{Ca}^{2+}$ signaling pathway in GABAergic neurons of the hippocampus. Proc. Natl. Acad. Sci. USA 1998, 95, 3245-3250. [CrossRef] [PubMed]

15. Juárez-Muñoz, Y.; Ramos-Languren, L.E.; Escobar, M.L. CaMKII Requirement for in Vivo Insular Cortex LTP Maintenance and CTA Memory Persistence. Front. Pharmacol. 2017, 8, 822. [CrossRef] [PubMed]

16. Achterberg, K.G.; Buitendijk, G.H.; Kool, M.J.; Goorden, S.M.; Post, L.; Slump, D.E.; Silva, A.J.; van Woerden, G.M.; Kushner, S.A.; Elgersma, Y. Temporal and region-specific requirements of $\alpha$ CaMKII in spatial and contextual learning. J. Neurosci. 2014, 34, 11180-11187. [CrossRef] [PubMed]

17. Silva, A.; Stevens, C.; Tonegawa, S.; Wang, Y. Deficient hippocampal long-term potentiation in alpha-calcium-calmodulin kinase II mutant mice. Science 1992, 257, 201-206. [CrossRef] [PubMed]

18. E Lisman, J.; Schulman, H.; Cline, H.T. The molecular basis of CaMKII function in synaptic and behavioural memory. Nat. Rev. Neurosci. 2002, 3, 175-190. [CrossRef]

19. Lucchesi, W.; Mizuno, K.; Giese, K.P. Novel insights into CaMKII function and regulation during memory formation. Brain Res. Bull. 2011, 85, 2-8. [CrossRef] [PubMed]

20. Dann, E.; Henderson, N.; Teichmann, S.; Morgan, M.; Marioni, J. Milo: Differential abundance testing on single-cell data using k-NN graphs. bioRxiv 2020. [CrossRef]

21. Chatzi, C.; Schnell, E.; Westbrook, G.L. Author response: Localized hypoxia within the subgranular zone determines the early survival of newborn hippocampal granule cells. eLife 2015, 4, 08722. [CrossRef]

22. Yamasaki, N.; Maekawa, M.; Kobayashi, K.; Kajii, Y.; Maeda, J.; Soma, M.; Takao, K.; Tanda, K.; Ohira, K.; Toyama, K.; et al. Alpha-CaMKII deficiency causes immature dentate gyrus, a novel candidate endophenotype of psychiatric disorders. Mol. Brain 2008, 1, 6. [CrossRef] [PubMed]

23. Vieira, H.L.; Alves, P.M.; Vercelli, A. Modulation of neuronal stem cell differentiation by hypoxia and reactive oxygen species. Prog. Neurobiol. 2011, 93, 444-455. [CrossRef] [PubMed]

24. Zhu, L.-L.; Wu, L.-Y.; Yew, D.T.; Fan, M. Effects of Hypoxia on the Proliferation and Differentiation of NSCs. Mol. Neurobiol. 2005, 31, 231-242. [CrossRef]

25. Večeřa, J.; Procházková, J.; Šumberová, V.; Pánská, V.; Paculová, H.; Lánová, M.K.; Mašek, J.; Bohačiaková, D.; Andersson, E.R.; Pacherník, J. Hypoxia/Hif1 $\alpha$ prevents premature neuronal differentiation of neural stem cells through the activation of Hes1. Stem Cell Res. 2020, 45, 101770. [CrossRef] [PubMed]

26. McClendon, E.; Shaver, D.C.; Degener-O’Brien, K.; Gong, X.; Nguyen, T.; Hoerder-Suabedissen, A.; Molnár, Z.; Mohr, C.; Richardson, B.D.; Rossi, D.J.; et al. Transient Hypoxemia Chronically Disrupts Maturation of Preterm Fetal Ovine Subplate Neuron Arborization and Activity. J. Neurosci. 2017, 37, 11912-11929. [CrossRef] [PubMed]

27. Li, Y.; Zhang, Y.; Liu, X.; Wang, M.; Wang, P.; Yang, J.; Zhang, S. Lutein inhibits proliferation, invasion and migration of hypoxic breast cancer cells via downregulation of HES1. Int. J. Oncol. 2018, 52, 2119-2129. [CrossRef]

28. Khuu, M.A.; Pagan, C.M.; Nallamothu, T.; Hevner, R.F.; Hodge, R.D.; Ramirez, J.-M.; Garcia, A.J. Intermittent Hypoxia Disrupts Adult Neurogenesis and Synaptic Plasticity in the Dentate Gyrus. J. Neurosci. 2018, 39, 1320-1331. [CrossRef]

29. Nallamothu, T.; Castro-Rivera, C.; Vera, A.; Garcia, A.J. Changes in Cellular Composition of Dentate Gyrus in Response to Intermittent Hypoxia. FASEB J. 2020, 34, 1. [CrossRef]

30. Constanthin, P.E.; Contestabile, A.; Petrenko, V.; Quairiaux, C.; Salmon, P.; Hüppi, P.S.; Kiss, J.Z. Endogenous erythropoietin signaling regulates migration and laminar positioning of upper-layer neurons in the developing neocortex. Development 2020, 147, dev190249. [CrossRef]

31. Noguchi, C.T.; Asavaritikrai, P.; Teng, R.; Jia, Y. Role of erythropoietin in the brain. Crit. Rev. Oncol. 2007, 64, 159-171. [CrossRef]

32. Lee, P.; Chandel, N.S.; Simon, M.C. Cellular adaptation to hypoxia through hypoxia inducible factors and beyond. Nat. Rev. Mol. Cell Biol. 2020, 21, 268-283. [CrossRef]

33. Jain, I.H.; Calvo, S.E.; Markhard, A.L.; Skinner, O.S.; To, T.-L.; Ast, T.; Mootha, V.K. Genetic Screen for Cell Fitness in High or Low Oxygen Highlights Mitochondrial and Lipid Metabolism. Cell 2020, 181, 716-727.e11. [CrossRef]

34. Sargin, D.; El-Kordi, A.; Agarwal, A.; Müller, M.; Wojcik, S.M.; Hassouna, I.; Sperling, S.; Nave, K.-A.; Ehrenreich, H. Expression of constitutively active erythropoietin receptor in pyramidal neurons of cortex and hippocampus boosts higher cognitive functions in mice. BMC Biol. 2011, 9, 27. [CrossRef]

35. Yoshimura, A.; Longmore, G.; Lodish, H.F. Point mutation in the exoplasmic domain of the erythropoietin receptor resulting in hormone-independent activation and tumorigenicity. Nat. Cell Biol. 1990, 348, 647-649. [CrossRef] [PubMed]

36. McKenzie, I.A.; Ohayon, D.; Li, H.; De Faria, J.P.; Emery, B.; Tohyama, K.; Richardson, W.D. Motor skill learning requires active central myelination. Science 2014, 346, 318-322. [CrossRef] [PubMed]

37. Liebetanz, D.; Merkler, D. Effects of commissural de- and remyelination on motor skill behaviour in the cuprizone mouse model of multiple sclerosis. Exp. Neurol. 2006, 202, 217-224. [CrossRef]

38. Liebmann, T.; Renier, N.; Bettayeb, K.; Greengard, P.; Tessier-Lavigne, M.; Flajolet, M. Three-Dimensional Study of Alzheimer's Disease Hallmarks Using the iDISCO Clearing Method. Cell Rep. 2016, 16, 1138-1152. [CrossRef] [PubMed]

39. Lein, E.S.; Hawrylycz, M.J.; Ao, N.; Ayres, M.; Bensinger, A.; Bernard, A.; Boe, A.F.; Boguski, M.S.; Brockway, K.S.; Byrnes, E.J.; et al. Genome-wide atlas of gene expression in the adult mouse brain. Nat. Cell Biol. 2006, 445, 168-176. [CrossRef]

40. R Core Team. R: A Language and Environment for Statistical Computing; R Foundation for Statistical Computing: Vienna, Austria, 2020. Available online: https://www.R-project.org (accessed on 18 February 2021). 
41. Stuart, T.; Butler, A.; Hoffman, P.; Hafemeister, C.; Papalexi, E.; Mauck, W.M.; Hao, Y.; Stoeckius, M.; Smibert, P.; Satija, R. Comprehensive Integration of Single-Cell Data. Cell 2019, 177, 1888-1902. [CrossRef] [PubMed]

42. Cembrowski, M.S.; Wang, L.; Sugino, K.; Shields, B.C.; Spruston, N. Hipposeq: A comprehensive RNA-seq database of gene expression in hippocampal principal neurons. eLife 2016, 5, e14997. [CrossRef] [PubMed]

43. Harris, K.D.; Hochgerner, H.; Skene, N.G.; Magno, L.; Katona, L.; Gonzales, C.B.; Somogyi, P.; Kessaris, N.; Linnarsson, S. Hjerling-Leffler, J. Classes and continua of hippocampal CA1 inhibitory neurons revealed by single-cell transcriptomics. PLoS Biol. 2018, 16, e2006387. [CrossRef] [PubMed]

44. Gu, Z.; Eils, R.; Schlesner, M. Complex heatmaps reveal patterns and correlations in multidimensional genomic data. Bioinformatics 2016, 32, 2847-2849. [CrossRef] [PubMed]

45. Chen, H. VennDiagram: Generate High-Resolution Venn and Euler Plots. R Package Version 1.6.20. 2018. Available online: https: / /CRAN.R-project.org/ package=VennDiagram (accessed on 18 February 2021). 


\section{Summary and conclusions}

Cellular homeostasis is critical for maintaining the physiological function of the body. In the central nervous system, constant supply and distribution of oxygen is essential for executing proper brain functions. Hypoxia has been implicated in fetal development and maintenance of hypoxic niches in the body (Giaccia et al., 2004; Mazumdar et al., 2010). On the contrary, it is also a potential driving force behind tumor growth, spread and worsened treatment outcomes. Hypoxia-inducible factors are central to the regulation of oxygen homeostasis as they are primarily responsible for generating compensatory responses under conditions of reduced oxygen (Semenza et al., 1991; Wang et al., 1995a). In the present work, we aimed to understand the multidimensional role of how the basic cellular oxygen sensing machinery works in the brain and what target transcriptional pathways are used when the machinery is activated while proceeding towards cellular homeostasis. Our data from newly generated hypoxia reporter mice provide new proof for a normal distribution of hypoxic cells along with functional implications of exercise and learning paradigms in the brain.

In chapter I, establishment and preliminary characterization of hypoxia reporter mouse lines are described. Initial generation of transgenic mice faced the problem of transgene silencing due to the presence of bacterial sequence in the plasmid. This issue was solved by removal of ampicillin resistance cassette from the plasmid to avoid the possible transgene silencing in the genome. Moreover, characterization experiments in CAG-CreERT2-ODD::R26R-tdTomato transgenic mice at early and old age provided the evidence of tamoxifen independent labelling of cells due to old age.

In chapter II, detailed results from basic characterization and validation of CAGCreERT2-ODD::R26R-tdTomato transgenic mice are presented. The generation of transgenic mice expressing CAG promoter allows the labelling of all hypoxic cells in the brain with tdTomato fluorescent protein, therefore establishing the basis for extensive investigation, of the homeostatic association with hypoxia in the body (Kimura et al., 2015). Currently different hypoxia reporting tools have been described, indicating the labelling of hypoxia in several in vitro and animal studies. These tools have practical and functional shortcomings in detecting low 
oxygen levels together with invasive of the approaches (Papkovsky \& Dmitriev, 2018). Our models provide ease of performing experiments as tamoxifen is injected peripherally and does not involve high grade invasive procedures for visualizing the hypoxic cells (Feil et al., 1997).

Evaluation of the oxygen-dependent regulation of the transgene by application of exogenous hypoxia $\left(6 \% \mathrm{O}_{2}\right.$ for $6 \mathrm{~h}$ ) in CAG-CreERT2-ODD::R26R-tdTomato mice, reveals the response by an increase in numbers of hypoxic cells. These findings follow a similar pattern in heart tissue, revealing the sensitivity and broad application of reporter systems (Kimura et al., 2015). In particular, detection of hypoxic cells under normoxic conditions in the brain was unexpected, although it is known that oxygen levels are low in the deeper regions of the brain (Silver et al., 1998). Along these lines, the expression of HIF-1 $\alpha$ under normoxic conditions in different organs and significant upregulation upon systemic hypoxia application are reported (Stroka et al., 2001). Taken together, these results point to low levels of oxygen in the brain, distinctly shown by tdTomato+ signal, and highlight the further use of these mice in long-term fate mapping under physiological conditions.

Indirect measurement of HIF-1a stabilization and cre activity by immunohistochemistry were not achieved due to the lack of suitable and specific antibodies. Apart from that, we tested the tissue hypoxia marker Pimonidazole (Hypoxyprobe-1 Mab1) and observed a few cells co-localizing tdTomato+ and pimonidazole staining in hypoxic cells (Sato et al., 2011). On the other hand, ligand-independent cre activation and spontaneous activity labelled hypoxic cells in transgenic mice were very low (Kristianto et al., 2017).

Data obtained from the oxygen and tamoxifen titration curves demonstrated a direct correlation with the total number of tdTomato+ hypoxic cells. Oxygen-severity results clearly indicate that lowering the oxygen level in the environment corresponds to the amount of hypoxic cells in the brain, revealing the transgene sensitivity to low oxygen. Previous studies have shown an organ-specific upregulation of HIF-1a in immunoblot analyses in tissues from different systemic hypoxia treated mice. Our observation confirms the results observed by Stroka et al. (1998), that a stepwise decrease in oxygen levels increases the amount of 
HIF-1 $\alpha$ in the brain. Furthermore, our data suggest enhanced cre-mediated recombination upon increasing tamoxifen injections, which is explained by high amounts of tamoxifen availability in the system.

Subsequent, immuno-histochemical results reveal distinct populations of hypoxic cells in the brain including neurons, astrocytes, oligodendrocytes, pericytes and endothelial cells. We noted that the neuronal population is the most vulnerable to reduced oxygen levels. It is well established from previous studies, that the hippocampus is most prone in stroke and ischemic conditions due to reduced oxygen availability (Schmidt-Kastner et al., 1991; Sommer, 1880). In this context, we observed a similar response in the hippocampus as the total number of hypoxic neurons significantly increased upon hypoxia application. Surprisingly, during histological examination we found no co-localization between IBA1+ and tdTomato+ fluorescence signals in the cells. This could be the result of hypoxic tolerance in microglia, as immune cells of the brain are not affected by reduced oxygen levels, or perhaps the absence of expression of the transgene. This peculiar response could be associated with developmental origin as during embryogenesis microglia are derived from mesoderm, later becoming part of the brain. (Chan et al., 2007; Obernier et al., 2019). This issue was addressed by confirming the active HIF machinery in the microglia by single cell RNA sequencing.

Additionally, our investigation in different hippocampal cell populations revealed strong hypoxic influences at mRNA level. This approach on first hand confirmed the presence and normal activity of both transgenes in all cells. These findings rule out the lack of transgene in microglial population. Differential gene expression analyses revealed strong upregulation of hypoxia related target genes in all cell clusters. This observation is in accordance with previous reports, demonstrating hypoxia-induced gene activity. Interestingly, high expression of Hk2 was observed under the normoxic and significantly upregulated under the hypoxic conditions. $H k 2$ is a glycolytic enzyme, highly active in anaerobic metabolism and is known to play an important role in tumor growth and progression (DeWaal et al., 2018; Li et al., 2018; Wolf et al., 2011). This could explain the absence of tdTomato+ expression in microglial population and providing hypoxic tolerance due to high expression of $H k 2$. 
Finally, we tested and confirmed our hypothesis that cognitive challenge by exercise and learning leads to functional hypoxia in the brain. Voluntary exercise on CRW lead to an increase in number of hypoxic neurons in CA1, CA3 and DG of CAG-CreERT2-ODD::R26R-tdTomato mice compared to sedentary controls. Exercise and learning tasks demand high oxygen content and glucose in the active area, due to high neuronal activity. Studies report that exposure to Morris water maze (MWM) and other exercise-related paradigms in mice resulted in the upregulation of HIF-1 $\alpha$ in the whole hippocampus, indicating the stabilization of HIF$1 \alpha$ (Kerr \& Swain, 2011; Wu et al., 2019). Exercise and learning tasks generate similar responses, as observed under hypoxic conditions to restore the homeostatic balance by transient episodes of functional hypoxia. These findings implicate that hypoxia is a critical part of brain cellular architecture and plays an important role in the plastic nature of hypoxic cells.

In light-sheet microscopy, systematic mapping of hypoxic cells revealed global distribution throughout the whole-brain. Some areas were highly sensitive to low oxygen levels and demonstrated high tdTomato+ cells under normal physiological conditions, including olfactory bulb and hippocampus. Quantification of total hypoxic cells in the cortical and hippocampal regions in the brain demonstrated significant differences in the hypoxia-treated mice compared to normoxic controls. 3D reconstruction results established overall spatial distributions and increases in the total number of hypoxic cells in the brain. Based on previous histological observations, total amount of hypoxic cells in the hippocampus was also significantly upregulated in mice exposed to CRW. These findings lay a groundwork for further investigations to address the involvement of hypoxia in physiological and pathological conditions.

Chapter III, earlier we observed that the neuronal population is most susceptible to hypoxic stimuli and a large subset of neurons undergoes hypoxia shown by tdTomato+ fluorescent protein. Therefore, we developed oxygen sensing reporter mice, which allow the detection and labelling of hypoxic neurons only in the brain. CaMKIla-CreERT2-ODD::R26R-tdTomato mice consist of inducible cre-recombinase fused to ODD domain of HIF-1a downstream of the neuron-specific CaMKIla promoter (Kimura et al., 2015; Mayford et al., 1996). Consequently, this 
reporter labels hypoxic neurons upon HIF-1 $\alpha$ stabilization with tdTomato+ fluorescent protein.

In a proof of principle experiment for basic characterization of CaMKIla-CreERT2ODD::R26R-tdTomato mice, the working efficiency was confirmed by application of exogenous hypoxia ( $6 \% \mathrm{O}_{2}$ for $6 \mathrm{~h}$ ), revealing a major population of neurons labelled by tdTomato+ fluorescent protein. This confirm the oxygen-dependent regulation of HIF-1a machinery. Interestingly, under normoxic conditions we observed a spare population of hypoxic neurons in the brain, similar to our previous findings in CAG expressing transgenic mice. Taken together, the present neuronspecific hypoxia reporter mice are an ideal and non-invasive tool to study hypoxia in neuronal populations.

Cellular morphology and identity was characterized in CaMKIla-CreERT2ODD::R26R-tdTomato mice by immune-staining with neuron-specific markers, demonstrating the overlapping signal tdTomato+/Ctip2+ in neuronal populations. The overall pattern of CaMKIla promoter expression is similar to previously published reports (Burgin et al., 1990; Mayford et al., 1996; Tsien et al., 1996). We observed an abundant expression of tdTomato+ fluorescent protein in the hippocampal and cortical region. The granular layer in dentate gyrus exhibited the highest number of tdTomato+ hypoxic neurons and strong tdTomato fluorescent signal rendering the cellular processes easily distinguishable. Particularly, in the stratum lucidum (SL), synaptic connections between mossy cells, granule cells and CA3 neurons display a strong tdTomato+ fluorescence signal. Remarkably, strong tdTomato expression in neurons allows the visualization of processes including dendrites, axons and, at a higher resolution, nerve endings and dendritic spines. Our results demonstrate that CaMKIla-CreERT2-ODD::R26R-tdTomato mice exhibit neuron-specific labelling of hypoxic cells and are suitable for mapping excitatory neuronal populations in the brain.

Cre-mediated recombination was evaluated by different tamoxifen injection paradigms in combination with exogenous hypoxia $\left(6 \% \mathrm{O}_{2}\right.$ for $\left.6 \mathrm{~h}\right)$ application in adult mice. We observed sparse labelling with a single tamoxifen dose and hypoxia exposure under hypoxic and normoxic conditions. In contrast, the number increased considerably upon accumulative tamoxifen injections in CA1, CA3 and 
DG of hippocampus. The severity of hypoxia and amount of ligand directly correlated with the total number of labelled hypoxic cells in the brain. Excess availability of ligand and hypoxia leads to saturation and abundant cre-mediated recombination in the brain. These findings confirm the sensitivity of transgene activity and suggest short-term treatment protocols to study physiological hypoxia in the brain.

We demonstrated the utility of CaMKIla-CreERT2-ODD::R26R-tdTomato under physiological settings by recording the effect of exercise and learning. Mice were allowed to freely run on the CRW for 5 days, which resulted in the upregulation of hypoxic cells in the hippocampus especially in females. This increase reflects exercise-induced neuronal-activity leading to HIF-1a stabilization and functional hypoxia, followed by presence of tdTomato+ protein in the neuronal population. Together with results of CRW in CAG-CreERT2-ODD mice, these data confirm the existence of exercise and learning-induced hypoxia in the brain.

Gender specific differences were observed in both transgenic mouse lines, in terms labelling of hypoxic neurons and response to hypoxic stimuli. In comparison to males, we have detected an enhanced response in females to hypoxia and CRW stimuli in the brain, represented overall by the total number of tdTomato+ hypoxic cells in the hippocampus. Previous studies have highlighted sex-based differences on treadmill running, demonstrating that females cover longer distances every day in comparison to males (Bono et al., 2006; Garland et al., 2011). Alternatively, variability in the number of tdTomato labelled hypoxic neurons could be explained by gender-based metabolic responses and bioavailability of tamoxifen concentration in the system (Wilson et al., 2016; Zampino et al., 2006).

In conclusion, the present thesis provides data comprising an improved understanding of hypoxia research in the brain. The development of new hypoxia reporter systems will provide the basis for a deeper understanding of the hypoxic machinery in physiological and pathological settings. In the future, these tools will allow spatial and temporal mapping of hypoxic cells, therefore promoting investigation of reduced oxygen levels in the brain. 


\section{Outlook and follow-up studies}

Collectively, the present research builds the groundwork for future hypoxia studies in the brain. Although current work addresses the issue of physiological hypoxia under normoxia and its increase upon complex motor learning tasks. However, it would be interesting to conduct follow-up studies:

To investigate, the functional consequence and validation of locally increased ODD stabilization and labelling of hypoxic cells in a different hypoxia reporting approaches.

To characterize and delineate the impact of NRW and CRW exposure on HIFbased hypoxia labelling and its association with cognitive improvement in trained and untrained mice.

To explore the impact of behavioral tests, which involve little or no exercise including environmental enrichment, fear conditioning and IntelliCage setups on the hypoxic state and cognitive improvement in the brain. 


\section{Bibliography}

Adamcio, B., Sargin, D., Stradomska, A., Medrihan, L., Gertler, C., Theis, F., et al. (2008). Erythropoietin enhances hippocampal long-term potentiation and memory. BMC Biology, 6(1), 37.

Adamcio, B., Sperling, S., Hagemeyer, N., Walkinshaw, G., \& Ehrenreich, H. (2010). Hypoxia inducible factor stabilization leads to lasting improvement of hippocampal memory in healthy mice. Behavioural Brain Research, 208(1), 80-84.

Ainslie, P. N., \& Ogoh, S. (2010). Regulation of cerebral blood flow in mammals during chronic hypoxia: a matter of balance. Experimental Physiology, 95(2), 251-262.

Appelhoff, R. J., Tian, Y.-M., Raval, R. R., Turley, H., Harris, A. L., Pugh, C. W., et al. (2004). Differential Function of the Prolyl Hydroxylases PHD1, PHD2, and PHD3 in the Regulation of Hypoxia-inducible Factor. Journal of Biological Chemistry, 279(37), 38458-38465.

Arany, Z., Huang, L. E., Eckner, R., Bhattacharya, S., Jiang, C., Goldberg, M. A., et al. (1996). An essential role for p300/CBP in the cellular response to hypoxia. Proceedings of the National Academy of Sciences, 93(23), 12969-12973.

Bélanger, M., Allaman, I., \& Magistretti, Pierre J. (2011). Brain Energy Metabolism: Focus on Astrocyte-Neuron Metabolic Cooperation. Cell Metabolism, 14(6), 724-738.

Berchtold, N. C., Castello, N., \& Cotman, C. W. (2010). Exercise and time-dependent benefits to learning and memory. Neuroscience, 167(3), 588-597.

Berra, E., Roux, D., Richard, D. E., \& Pouysségur, J. (2001). Hypoxia-inducible factor-1 $\alpha$ (HIF-1 $\alpha$ ) escapes O2-driven proteasomal degradation irrespective of its subcellular localization: nucleus or cytoplasm. EMBO reports, 2(7), 615-620.

Bhaskaran, K., Douglas, I., Forbes, H., dos-Santos-Silva, I., Leon, D. A., \& Smeeth, L. (2014). Bodymass index and risk of 22 specific cancers: a population-based cohort study of $5 \cdot 24$ million UK adults. The Lancet, 384(9945), 755-765.

Bloor, C. M. (2005). Angiogenesis during exercise and training. Angiogenesis, 8(3), 263-271.

Blouin, C. C., Pagé, E. L., Soucy, G. M., \& Richard, D. E. (2004). Hypoxic gene activation by lipopolysaccharide in macrophages: implication of hypoxia-inducible factor $1 \alpha$. Blood, 103(3), 1124-1130.

Bono, J. P. D., Adlam, D., Paterson, D. J., \& Channon, K. M. (2006). Novel quantitative phenotypes of exercise training in mouse models. American Journal of Physiology-Regulatory, Integrative and Comparative Physiology, 290(4), R926-R934.

Branda, C. S., \& Dymecki, S. M. (2004). Talking about a Revolution: The Impact of Site-Specific Recombinases on Genetic Analyses in Mice. Developmental Cell, 6(1), 7-28.

Brocato, J., Chervona, Y., \& Costa, M. (2014). Molecular responses to hypoxia-inducible factor $1 \alpha$ and beyond. Molecular pharmacology, 85(5), 651-657.

Bruick, R. K., \& McKnight, S. L. (2001). A Conserved Family of Prolyl-4-Hydroxylases That Modify HIF. Science, 294(5545), 1337-1340.

Burgin, K., Waxham, M., Rickling, S., Westgate, S., Mobley, W., \& Kelly, P. (1990). In situ hybridization histochemistry of $\mathrm{Ca} 2+/$ calmodulin-dependent protein kinase in developing rat brain. The Journal of Neuroscience, 10(6), 1788-1798.

Butler, A., Hoffman, P., Smibert, P., Papalexi, E., \& Satija, R. (2018). Integrating single-cell transcriptomic data across different conditions, technologies, and species. Nature Biotechnology, 36, 411-420.

Cao, L., Jiao, X., Zuzga, D. S., Liu, Y., Fong, D. M., Young, D., et al. (2004). VEGF links hippocampal activity with neurogenesis, learning and memory. Nature Genetics, 36(8), 827-835.

Carreau, A., Hafny-Rahbi, B. E., Matejuk, A., Grillon, C., \& Kieda, C. (2011). Why is the partial oxygen pressure of human tissues a crucial parameter? Small molecules and hypoxia. Journal of Cellular and Molecular Medicine, 15(6), 1239-1253.

Chan, W. Y., Kohsaka, S., \& Rezaie, P. (2007). The origin and cell lineage of microglia-New concepts. Brain Research Reviews, 53(2), 344-354. 
Chandel, N. S., \& Schumacker, P. T. (2000). Cellular oxygen sensing by mitochondria: old questions, new insight. Journal of Applied Physiology, 88(5), 1880-1889.

Chávez, J. C., Agani, F., Pichiule, P., \& LaManna, J. C. (2000). Expression of hypoxia-inducible factor- $1 \alpha$ in the brain of rats during chronic hypoxia. Journal of Applied Physiology, 89(5), 1937-1942.

Chen, L., Endler, A., \& Shibasaki, F. (2009). Hypoxia and angiogenesis: regulation of hypoxiainducible factors via novel binding factors. Experimental \&Amp; Molecular Medicine, 41 849.

Chen, Z., He, C., Meuse, L., \& Kay, M. (2004). Silencing of episomal transgene expression by plasmid bacterial DNA elements in vivo. Gene therapy, 11(10), 856.

Chiche, J., Ilc, K., Laferrière, J., Trottier, E., Dayan, F., Mazure, N. M., et al. (2009). HypoxiaInducible Carbonic Anhydrase IX and XII Promote Tumor Cell Growth by Counteracting Acidosis through the Regulation of the Intracellular pH. Cancer Research, 69(1), 358-368.

Chilov, D., Camenisch, G., Kvietikova, I., Ziegler, U., Gassmann, M., \& Wenger, R. H. (1999). Induction and nuclear translocation of hypoxia-inducible factor-1 (HIF-1): heterodimerization with ARNT is not necessary for nuclear accumulation of HIF-1alpha. Journal of Cell Science, 112(8), 1203-1212.

Chin, D., \& Means, A. R. (2000). Calmodulin: a prototypical calcium sensor. Trends in Cell Biology, 10(8), 322-328.

Chun, Y. S., Kim, M. S., \& Park, J. W. (2002). Oxygen-Dependent and -Independent Regulation of HIF-1alpha. J Korean Med Sci, 17(5), 581-588.

Cotman, C. W., Berchtold, N. C., \& Christie, L.-A. (2007). Exercise builds brain health: key roles of growth factor cascades and inflammation. Trends in Neurosciences, 30(9), 464-472.

Coultrap, S. J., \& Bayer, K. U. (2012). CaMKII regulation in information processing and storage. Trends in Neurosciences, 35(10), 607-618.

Danhier, P., Krishnamachary, B., Bharti, S., Kakkad, S., Mironchik, Y., \& Bhujwalla, Z. M. (2015). Combining Optical Reporter Proteins with Different Half-lives to Detect Temporal Evolution of Hypoxia and Reoxygenation in Tumors. Neoplasia, 17(12), 871-881.

Dann, C. E., Bruick, R. K., \& Deisenhofer, J. (2002). Structure of factor-inhibiting hypoxiainducible factor 1: An asparaginyl hydroxylase involved in the hypoxic response pathway. Proceedings of the National Academy of Sciences, 99(24), 15351-15356.

Dengler, V. L., Galbraith, M. D., \& Espinosa, J. M. (2014). Transcriptional regulation by hypoxia inducible factors. Critical Reviews in Biochemistry and Molecular Biology, 49(1), 1-15.

DeWaal, D., Nogueira, V., Terry, A. R., Patra, K. C., Jeon, S.-M., Guzman, G., et al. (2018). Hexokinase-2 depletion inhibits glycolysis and induces oxidative phosphorylation in hepatocellular carcinoma and sensitizes to metformin. Nature Communications, 9(1), 446.

Dunham-Snary, K. J., Hong, Z. G., Xiong, P. Y., Del Paggio, J. C., Herr, J. E., Johri, A. M., et al. (2016). A mitochondrial redox oxygen sensor in the pulmonary vasculature and ductus arteriosus. Pflügers Archiv - European Journal of Physiology, 468(1), 43-58.

Dunwoodie, S. L. (2009). The Role of Hypoxia in Development of the Mammalian Embryo. Developmental Cell, 17(6), 755-773.

Ehrenreich, H., Degner, D., Meller, J., Brines, M., Béhé, M., Hasselblatt, M., et al. (2004). Erythropoietin: a candidate compound for neuroprotection in schizophrenia. Molecular Psychiatry, 9(1), 42-54.

Ehrenreich, H., Fischer, B., Norra, C., Schellenberger, F., Stender, N., Stiefel, M., et al. (2007a). Exploring recombinant human erythropoietin in chronic progressive multiple sclerosis. Brain, 130(10), 2577-2588.

Ehrenreich, H., Hinze-Selch, D., Stawicki, S., Aust, C., Knolle-Veentjer, S., Wilms, S., et al. (2007b). Improvement of cognitive functions in chronic schizophrenic patients by recombinant human erythropoietin. Molecular Psychiatry, 12(2), 206. 
Ehrenreich, H., Weissenborn, K., Prange, H., Schneider, D., Weimar, C., Wartenberg, K., et al. (2009). Recombinant human erythropoietin in the treatment of acute ischemic stroke. Stroke, 40(12), e647-e656.

El-Kordi, A., Radyushkin, K., \& Ehrenreich, H. (2009). Erythropoietin improves operant conditioning and stability of cognitive performance in mice. BMC Biology, 7, 37-37.

Eltzschig, H. K., \& Carmeliet, P. (2011). Hypoxia and Inflammation. New England Journal of Medicine, 364(7), 656-665.

Ema, M., Taya, S., Yokotani, N., Sogawa, K., Matsuda, Y., \& Fujii-Kuriyama, Y. (1997). A novel bHLH-PAS factor with close sequence similarity to hypoxia-inducible factor $1 \alpha$ regulates the <em $>\mathrm{VEGF}</ \mathrm{em}>$ expression and is potentially involved in lung and vascular development. Proceedings of the National Academy of Sciences, 94(9), 42734278.

Engelhardt, S., Huang, S.-F., Patkar, S., Gassmann, M., \& Ogunshola, O. O. (2015). Differential responses of blood-brain barrier associated cells to hypoxia and ischemia: a comparative study. Fluids and barriers of the CNS, 12, 4-4.

Epstein, A. C. R., Gleadle, J. M., McNeill, L. A., Hewitson, K. S., O'Rourke, J., Mole, D. R., et al. (2001). C. elegans EGL-9 and Mammalian Homologs Define a Family of Dioxygenases that Regulate HIF by Prolyl Hydroxylation. Cell, 107(1), 43-54.

Erapaneedi, R., Belousov, V. V., Schäfers, M., \& Kiefer, F. (2016). A novel family of fluorescent hypoxia sensors reveal strong heterogeneity in tumor hypoxia at the cellular level. The EMBO Journal, 35(1), 102.

Erondu, N., \& Kennedy, M. (1985). Regional distribution of type II Ca2+/calmodulin-dependent protein kinase in rat brain. The Journal of Neuroscience, 5(12), 3270-3277.

Esipova, T. V., Barrett, M. J. P., Erlebach, E., Masunov, A. E., Weber, B., \& Vinogradov, S. A. (2019). Oxyphor 2P: A High-Performance Probe for Deep-Tissue Longitudinal Oxygen Imaging. Cell Metabolism, 29(3), 736-744.e737.

Fabel, K., \& Kempermann, G. (2008). Physical Activity and the Regulation of Neurogenesis in the Adult and Aging Brain. NeuroMolecular Medicine, 10(2), 59-66.

Faton, A., \& Bing-Hua, J. (2013). Oxygen-independent Regulation of HIF-1: Novel Involvement of $\mathrm{PI3K} / \mathrm{AKT} / \mathrm{mTOR}$ Pathway in Cancer. Current Cancer Drug Targets, 13(3), 245-251.

Favret, F., \& Richalet, J.-P. (2007). Exercise and hypoxia: The role of the autonomic nervous system. Respiratory Physiology \& Neurobiology, 158(2), 280-286.

Feil, R., Wagner, J., Metzger, D., \& Chambon, P. (1997). Regulation of Cre Recombinase Activity by Mutated Estrogen Receptor Ligand-Binding Domains. Biochemical and Biophysical Research Communications, 237(3), 752-757.

Garland, T., Kelly, S. A., Malisch, J. L., Kolb, E. M., Hannon, R. M., Keeney, B. K., et al. (2011). How to run far: multiple solutions and sex-specific responses to selective breeding for high voluntary activity levels. Proceedings of the Royal Society B: Biological Sciences, 278(1705), 574-581.

Giaccia, A. J., Simon, M. C., \& Johnson, R. (2004). The biology of hypoxia: the role of oxygen sensing in development, normal function, and disease. Genes \& development, 18(18), 2183-2194.

Goldman, S. J., Chen, E., Taylor, R., Zhang, S., Petrosky, W., Reiss, M., et al. (2011). Use of the ODD-Luciferase Transgene for the Non-Invasive Imaging of Spontaneous Tumors in Mice. PloS one, 6(3), e18269.

Goodall, S., Twomey, R., \& Amann, M. (2014). Acute and chronic hypoxia: implications for cerebral function and exercise tolerance. Fatigue : biomedicine, health \& behavior, 2(2), 73-92.

Gorres, K. L., \& Raines, R. T. (2010). Prolyl 4-hydroxylase. Critical Reviews in Biochemistry and Molecular Biology, 45(2), 106-124. 
Gould, E., Reeves, A. J., Fallah, M., Tanapat, P., Gross, C. G., \& Fuchs, E. (1999a). Hippocampal neurogenesis in adult Old World primates. Proceedings of the National Academy of Sciences of the United States of America, 96(9), 5263-5267.

Gould, E., Reeves, A. J., Graziano, M. S. A., \& Gross, C. G. (1999b). Neurogenesis in the Neocortex of Adult Primates. Science, 286(5439), 548-552.

Gourine, A. V. (2005). On the peripheral and central chemoreception and control of breathing: an emerging role of ATP. The Journal of Physiology, 568(3), 715-724.

Gu, Y. Z., Moran, S. M., Hogenesch, J. B., Wartman, L., \& Bradfield, C. A. (1998). Molecular characterization and chromosomal localization of a third alpha-class hypoxia inducible factor subunit, HIF3alpha. Gene expression, 7(3), 205-213.

Guyenet, Patrice G., \& Bayliss, Douglas A. (2015). Neural Control of Breathing and CO2 Homeostasis. Neuron, 87(5), 946-961.

Hara, H., Onodera, H., Kyuya, K., \& Akaike, N. (1990). The regional difference of neuronal susceptibility in the dentate gyrus to hypoxia. Neuroscience Letters, 115(2), 189-194.

Harris, K. D., Hochgerner, H., Skene, N. G., Magno, L., Katona, L., Bengtsson Gonzales, C., et al. (2018). Classes and continua of hippocampal CA1 inhibitory neurons revealed by singlecell transcriptomics. PLOS Biology, 16(6), e2006387.

Hassouna, I., Ott, C., Wüstefeld, L., Offen, N., Neher, R. A., Mitkovski, M., et al. (2016). Revisiting adult neurogenesis and the role of erythropoietin for neuronal and oligodendroglial differentiation in the hippocampus. Molecular Psychiatry, 21(12), 1752-1767.

Hewitson, K. S., McNeill, L. A., Riordan, M. V., Tian, Y.-M., Bullock, A. N., Welford, R. W., et al. (2002). Hypoxia-inducible Factor (HIF) Asparagine Hydroxylase Is Identical to Factor Inhibiting HIF (FIH) and Is Related to the Cupin Structural Family. Journal of Biological Chemistry, 277(29), 26351-26355.

Hillman, C. H., Erickson, K. I., \& Kramer, A. F. (2008). Be smart, exercise your heart: exercise effects on brain and cognition. Nature Reviews Neuroscience, 9, 58.

Hochgerner, H., Zeisel, A., Lönnerberg, P., \& Linnarsson, S. (2018). Conserved properties of dentate gyrus neurogenesis across postnatal development revealed by single-cell RNA sequencing. Nature Neuroscience, 21(2), 290-299.

Hsia, C. C. W., Schmitz, A., Lambertz, M., Perry, S. F., \& Maina, J. N. (2013). Evolution of air breathing: oxygen homeostasis and the transitions from water to land and sky. Comprehensive Physiology, 3(2), 849-915.

Hu, C.-J., Sataur, A., Wang, L., Chen, H., \& Simon, M. C. (2007). The N-Terminal Transactivation Domain Confers Target Gene Specificity of Hypoxia-inducible Factors HIF-1 $\alpha$ and HIF-2 $\alpha$. Molecular Biology of the Cell, 18(11), 4528-4542.

Huang, L. E., Arany, Z., Livingston, D. M., \& Bunn, H. F. (1996). Activation of hypoxia-inducible transcription factor depends primarily upon redox-sensitive stabilization of its $\alpha$ subunit. Journal of Biological Chemistry, 271(50), 32253-32259.

Huang, L. E., Gu, J., Schau, M., \& Bunn, H. F. (1998). Regulation of hypoxia-inducible factor $1 \alpha$ is mediated by an O2-dependent degradation domain via the ubiquitin-proteasome pathway. Proceedings of the National Academy of Sciences, 95(14), 7987-7992.

Ivan, M., Kondo, K., Yang, H., Kim, W., Valiando, J., Ohh, M., et al. (2001). HIF $\alpha$ Targeted for VHLMediated Destruction by Proline Hydroxylation: Implications for O2 Sensing. Science, 292(5516), 464-468.

lyer, N. V., Kotch, L. E., Agani, F., Leung, S. W., Laughner, E., Wenger, R. H., et al. (1998). Cellular and developmental control of $\mathrm{O} 2$ homeostasis by hypoxia-inducible factor $1 \alpha$. Genes \& development, 12(2), 149-162.

Jankowsky, J. L., Melnikova, T., Fadale, D. J., Xu, G. M., Slunt, H. H., Gonzales, V., et al. (2005). Environmental Enrichment Mitigates Cognitive Deficits in a Mouse Model of Alzheimer's Disease. The Journal of Neuroscience, 25(21), 5217-5224.

Jelkmann, W. (2004). Molecular Biology of Erythropoietin. Internal Medicine, 43(8), 649-659. 
Jeong, J.-W., Bae, M.-K., Ahn, M.-Y., Kim, S.-H., Sohn, T.-K., Bae, M.-H., et al. (2002). Regulation and Destabilization of HIF-1 $\alpha$ by ARD1-Mediated Acetylation. Cell, 111(5), 709-720.

Jiang, B.-H., Rue, E., Wang, G. L., Roe, R., \& Semenza, G. L. (1996). Dimerization, DNA Binding, and Transactivation Properties of Hypoxia-inducible Factor 1. Journal of Biological Chemistry, 271(30), 17771-17778.

Jin, K., Zhu, Y., Sun, Y., Mao, X. O., Xie, L., \& Greenberg, D. A. (2002). Vascular endothelial growth factor (VEGF) stimulates neurogenesis in vitro and in vivo. Proceedings of the National Academy of Sciences of the United States of America, 99(18), 11946-11950.

Kaelin, W. G., \& Ratcliffe, P. J. (2008). Oxygen sensing by metazoans: The central role of the HIF hydroxylase pathway. Molecular Cell, 30(4), 393-402.

Kallio, P. J., Okamoto, K., O'Brien, S., Carrero, P., Makino, Y., Tanaka, H., et al. (1998). Signal transduction in hypoxic cells: inducible nuclear translocation and recruitment of theCBP/p300 coactivator by the hypoxia-induciblefactor-1 $\alpha$. The EMBO Journal, 17(22), 6573-6586.

Kamura, T., Sato, S., Iwai, K., Czyzyk-Krzeska, M., Conaway, R. C., \& Conaway, J. W. (2000). Activation of HIF1alpha ubiquitination by a reconstituted von Hippel-Lindau (VHL) tumor suppressor complex. Proceedings of the National Academy of Sciences of the United States of America, 97(19), 10430-10435.

Kenneth, N. S., \& Rocha, S. (2008). Regulation of gene expression by hypoxia. Biochemical Journal, 414(1), 19-29.

Kerr, A. L., \& Swain, R. A. (2011). Rapid cellular genesis and apoptosis: Effects of exercise in the adult rat. Behavioral Neuroscience, 125(1), 1-9.

Khurana, P., Sugadev, R., Jain, J., \& Singh, S. B. (2013). HypoxiaDB: a database of hypoxiaregulated proteins. Database, 2013.

Kimura, W., Xiao, F., Canseco, D. C., Muralidhar, S., Thet, S., Zhang, H. M., et al. (2015). Hypoxia fate mapping identifies cycling cardiomyocytes in the adult heart. Nature, 523(7559), 226-230.

Kisler, K., Nelson, A. R., Rege, S. V., Ramanathan, A., Wang, Y., Ahuja, A., et al. (2017). Pericyte degeneration leads to neurovascular uncoupling and limits oxygen supply to brain. Nature Neuroscience, 20(3), 406-416.

Koh, M. Y., \& Powis, G. (2012). Passing the baton: the HIF switch. Trends in Biochemical Sciences, $37(9), 364-372$.

Koivunen, P., Tiainen, P., Hyvärinen, J., Williams, K. E., Sormunen, R., Klaus, S. J., et al. (2007). An Endoplasmic Reticulum Transmembrane Prolyl 4-Hydroxylase Is Induced by Hypoxia and Acts on Hypoxia-inducible Factor $\alpha$. Journal of Biological Chemistry, 282(42), 3054430552.

Kriegstein, A., \& Alvarez-Buylla, A. (2009). The Glial Nature of Embryonic and Adult Neural Stem Cells. Annual Review of Neuroscience, 32(1), 149-184.

Kristianto, J., Johnson, M. G., Zastrow, R. K., Radcliff, A. B., \& Blank, R. D. (2017). Spontaneous recombinase activity of Cre-ERT2 in vivo. Transgenic Research, 26(3), 411-417.

Kumar, P., \& Prabhakar, N. R. (2012). Peripheral chemoreceptors: function and plasticity of the carotid body. Comprehensive Physiology, 2(1), 141-219.

Kump, L. R. (2008). The rise of atmospheric oxygen. Nature, 451(7176), 277.

Lahiri, S., Baby, S., DiGiulio, C., \& Roy, A. (2007). CO2/H+ Homeostasis: Role of Central and Peripheral Chemoreceptors in Adult Mammals (pp. 1-15).

Lahiri, S., \& Forster, R. E. (2003). CO2/H+ sensing: peripheral and central chemoreception. The International Journal of Biochemistry \& Cell Biology, 35(10), 1413-1435.

Laitala, A., Aro, E., Walkinshaw, G., Mäki, J. M., Rossi, M., Heikkilä, M., et al. (2012). Transmembrane prolyl 4-hydroxylase is a fourth prolyl 4-hydroxylase regulating EPO production and erythropoiesis. Blood, 120(16), 3336-3344. 
Lando, D., Peet, D. J., Gorman, J. J., Whelan, D. A., Whitelaw, M. L., \& Bruick, R. K. (2002). FIH-1 is an asparaginyl hydroxylase enzyme that regulates the transcriptional activity of hypoxia-inducible factor. Genes \& development, 16(12), 1466-1471.

Lee, I. M., Shiroma, E. J., Lobelo, F., Puska, P., Blair, S. N., \& Katzmarzyk, P. T. (2012). Effect of physical inactivity on major non-communicable diseases worldwide: an analysis of burden of disease and life expectancy. The Lancet, 380(9838), 219-229.

Lee, K. E., \& Simon, M. C. (2015). SnapShot: Hypoxia-Inducible Factors. Cell, 163(5), 12881288.e1281.

Lewczuk, P., Hasselblatt, M., Kamrowski-Kruck, H., Heyer, A., Unzicker, C., Sirén, A. L., et al. (2000). Survival of hippocampal neurons in culture upon hypoxia: effect of erythropoietin. Neuroreport, 11(16), 3485-3488.

Lewis, L. D., Pontén, U., \& Siesjö, B. K. (1973). Homeostatic Regulation of Brain Energy Metabolism in Hypoxia. Acta Physiologica Scandinavica, 88(2), 284-286.

Li, Y., Lu, B., Sheng, L., Zhu, Z., Sun, H., Zhou, Y., et al. (2018). Hexokinase 2-dependent hyperglycolysis driving microglial activation contributes to ischemic brain injury. Journal of Neurochemistry, 144(2), 186-200.

Lidsky, P. V., Lukyanov, K. A., Misra, T., Handke, B., Mishin, A. S., \& Lehner, C. F. (2018). A genetically encoded fluorescent probe for imaging of oxygenation gradients in living \&lt;em\&gt;Drosophila\&lt;/em\&gt. Development, 145(4), dev156257.

Lisman, J., Schulman, H., \& Cline, H. (2002). The molecular basis of CaMKII function in synaptic and behavioural memory. Nature Reviews Neuroscience, 3(3), 175-190.

Liu, P. Z., \& Nusslock, R. (2018). Exercise-Mediated Neurogenesis in the Hippocampus via BDNF. Frontiers in Neuroscience, 12(52).

Louie, E., Nik, S., Chen, J.-s., Schmidt, M., Song, B., Pacson, C., et al. (2010). Identification of a stem-like cell population by exposing metastatic breast cancer cell lines to repetitive cycles of hypoxia and reoxygenation. Breast Cancer Research, 12(6), R94.

Mahon, P. C., Hirota, K., \& Semenza, G. L. (2001). FIH-1: a novel protein that interacts with HIF1alpha and VHL to mediate repression of HIF-1 transcriptional activity. Genes \& development, 15(20), 2675-2686.

Makino, Y., Cao, R., Svensson, K., Bertilsson, G., Asman, M., Tanaka, H., et al. (2001). Inhibitory PAS domain protein is a negative regulator of hypoxia-inducible gene expression. Nature, 414(6863), 550-554.

Makino, Y., Kanopka, A., Wilson, W. J., Tanaka, H., \& Poellinger, L. (2002). Inhibitory PAS Domain Protein (IPAS) Is a Hypoxia-inducible Splicing Variant of the Hypoxia-inducible Factor- $3 \alpha$ Locus. Journal of Biological Chemistry, 277(36), 32405-32408.

Marti, H. J. H., Bernaudin, M., Bellail, A., Schoch, H., Euler, M., Petit, E., et al. (2000). HypoxiaInduced Vascular Endothelial Growth Factor Expression Precedes Neovascularization after Cerebral Ischemia. The American Journal of Pathology, 156(3), 965-976.

Marxsen, J. H., Stengel, P., Doege, K., Heikkinen, P., Jokilehto, T., Wagner, T., et al. (2004). Hypoxia-inducible factor-1 (HIF-1) promotes its degradation by induction of HIF-alphaprolyl-4-hydroxylases. The Biochemical journal, 381(Pt 3), 761-767.

Masoud, G. N., \& Li, W. (2015). HIF-1 $\alpha$ pathway: role, regulation and intervention for cancer therapy. Acta Pharmaceutica Sinica B, 5(5), 378-389.

Maxwell, P. H., Dachs, G. U., Gleadle, J. M., Nicholls, L. G., Harris, A. L., Stratford, I. J., et al. (1997) Hypoxia-inducible factor-1 modulates gene expression in solid tumors and influences both angiogenesis and tumor growth. Proceedings of the National Academy of Sciences of the United States of America, 94(15), 8104-8109.

Mayford, M., Bach, M. E., Huang, Y.-Y., Wang, L., Hawkins, R. D., \& Kandel, E. R. (1996). Control of Memory Formation Through Regulated Expression of a CaMKII Transgene. Science, 274(5293), 1678-1683. 
Mazumdar, J., O'Brien, W. T., Johnson, R. S., LaManna, J. C., Chavez, J. C., Klein, P. S., et al. (2010). $\mathrm{O} 2$ regulates stem cells through $\mathrm{Wnt} / \beta$-catenin signalling. Nature cell biology, 12(10), 1007-1013.

McEachern, L. A. (2012). Transgenic Epigenetics: Using Transgenic Organisms to Examine Epigenetic Phenomena. Genetics Research International, 2012, 14.

McKenzie, I. A., Ohayon, D., Li, H., Paes de Faria, J., Emery, B., Tohyama, K., et al. (2014). Motor skill learning requires active central myelination. Science, 346(6207), 318-322.

McNeill, L. A., Hewitson, K. S., Gleadle, J. M., Horsfall, L. E., Oldham, N. J., Maxwell, P. H., et al. (2002). The use of dioxygen by HIF prolyl hydroxylase (PHD1). Bioorganic \& Medicinal Chemistry Letters, 12(12), 1547-1550.

Meijer, J. H., \& Robbers, Y. (2014). Wheel running in the wild. Proceedings of the Royal Society B: Biological Sciences, 281(1786), 20140210.

Mergenthaler, P., Lindauer, U., Dienel, G. A., \& Meisel, A. (2013). Sugar for the brain: the role of glucose in physiological and pathological brain function. Trends in Neurosciences, 36(10), 587-597.

Metzger, D., \& Chambon, P. (2001). Site- and Time-Specific Gene Targeting in the Mouse. Methods, 24(1), 71-80.

Michiels, C. (2004). Physiological and pathological responses to hypoxia. The American Journal of Pathology, 164(6), 1875-1882.

Minichiello, L., Korte, M., Wolfer, D., Kühn, R., Unsicker, K., Cestari, V., et al. (1999). Essential Role for TrkB Receptors in Hippocampus-Mediated Learning. Neuron, 24(2), 401-414.

Mirabello, V., Cortezon-Tamarit, F., \& Pascu, S. I. (2018). Oxygen Sensing, Hypoxia Tracing and in Vivo Imaging with Functional Metalloprobes for the Early Detection of Noncommunicable Diseases. Frontiers in chemistry, 6, 27-27.

Misra, T., Baccino-Calace, M., Meyenhofer, F., Rodriguez-Crespo, D., Akarsu, H., ArmentaCalderón, R., et al. (2016). A genetically encoded biosensor for visualizing hypoxia responses in vivo. Biology Open, bio.018226.

Mohyeldin, A., Garzón-Muvdi, T., \& Quiñones-Hinojosa, A. (2010). Oxygen in Stem Cell Biology: A Critical Component of the Stem Cell Niche. Cell Stem Cell, 7(2), 150-161.

Myllyharju, J., \& Kivirikko, K. I. (1997). Characterization of the iron- and 2-oxoglutarate-binding sites of human prolyl 4-hydroxylase. The EMBO Journal, 16(6), 1173-1180.

Nakada, Y., Canseco, D. C., Thet, S., Abdisalaam, S., Asaithamby, A., Santos, C. X., et al. (2016). Hypoxia induces heart regeneration in adult mice. Nature, 541, 222-227.

O'Regan, R. G., \& Majcherczyk, S. (1982). Role of peripheral chemoreceptors and central chemosensitivity in the regulation of respiration and circulation. Journal of Experimental Biology, 100(1), 23-40.

Obernier, K., \& Alvarez-Buylla, A. (2019). Neural stem cells: origin, heterogeneity and regulation in the adult mammalian brain. Development, 146(4), dev156059.

Ogoh, S., \& Ainslie, P. N. (2009). Cerebral blood flow during exercise: mechanisms of regulation. Journal of Applied Physiology, 107(5), 1370-1380.

Papkovsky, D. B., \& Dmitriev, R. I. (2018). Imaging of oxygen and hypoxia in cell and tissue samples. Cellular and Molecular Life Sciences, 75(16), 2963-2980.

Parmar, K., Mauch, P., Vergilio, J.-A., Sackstein, R., \& Down, J. D. (2007). Distribution of hematopoietic stem cells in the bone marrow according to regional hypoxia. Proceedings of the National Academy of Sciences, 104(13), 5431-5436.

Peng, J., Zhang, L., Drysdale, L., \& Fong, G.-H. (2000). The transcription factor EPAS-1/hypoxiainducible factor $2 \alpha$ plays an important role in vascular remodeling. Proceedings of the National Academy of Sciences, 97(15), 8386-8391.

Prabhakar, N. R., \& Peng, Y.-J. (2004). Peripheral chemoreceptors in health and disease. Journal of Applied Physiology, 96(1), 359-366. 
Pugh, C. W., O'Rourke, J. F., Nagao, M., Gleadle, J. M., \& Ratcliffe, P. J. (1997). Activation of Hypoxia-inducible Factor-1; Definition of Regulatory Domains within the $\alpha$ Subunit. Journal of Biological Chemistry, 272(17), 11205-11214.

Querido, J. S., \& Sheel, A. W. (2007). Regulation of Cerebral Blood Flow During Exercise. Sports Medicine, 37(9), 765-782.

Ratcliffe, P. J. (2013). Oxygen sensing and hypoxia signalling pathways in animals: the implications of physiology for cancer. The Journal of Physiology, 591(8), 2027-2042.

Ravenna, L., Salvatori, L., \& Russo, M. A. (2016). HIF3 $\alpha$ : the little we know. The FEBS Journal, 283(6), 993-1003.

Roh, E., Song, D. K., \& Kim, M.-S. (2016). Emerging role of the brain in the homeostatic regulation of energy and glucose metabolism. Experimental \& molecular medicine, 48(3), e216e216.

Ruscher, K., Isaev, N., Trendelenburg, G., Weih, M., Iurato, L., Meisel, A., et al. (1998). Induction of hypoxia inducible factor 1 by oxygen glucose deprivation is attenuated by hypoxic preconditioning in rat cultured neurons. Neuroscience Letters, 254(2), 117-120.

Safran, M., Kim, W. Y., Connell, F., Flippin, L., Günzler, V., Horner, J. W., et al. (2006). Mouse model for noninvasive imaging of HIF prolyl hydroxylase activity: Assessment of an oral agent that stimulates erythropoietin production. Proceedings of the National Academy of Sciences of the United States of America, 103(1), 105.

Salceda, S., \& Caro, J. (1997). Hypoxia-inducible Factor $1 \alpha$ (HIF-1 $\alpha$ ) Protein Is Rapidly Degraded by the Ubiquitin-Proteasome System under Normoxic Conditions: ITS STABILIZATION BY HYPOXIA DEPENDS ON REDOX-INDUCED CHANGES. Journal of Biological Chemistry, 272(36), 22642-22647.

Sandau, K. B., Fandrey, J., \& Brüne, B. (2001). Accumulation of HIF-1 $\alpha$ under the influence of nitric oxide. Blood, 97(4), 1009-1015.

Sandhu, S., Kydd, L., \& Jaworski, J. (2017). Luminescent Probe Based Techniques for Hypoxia Imaging. Journal of nanomedicine research, 6(3), 00160.

Sargin, D., El-Kordi, A., Agarwal, A., Müller, M., Wojcik, S. M., Hassouna, I., et al. (2011). Expression of constitutively active erythropoietin receptor in pyramidal neurons of cortex and hippocampus boosts higher cognitive functions in mice. BMC Biology, 9(1), 27

Sato, Y., Endo, H., Okuyama, H., Takeda, T., Iwahashi, H., Imagawa, A., et al. (2011). Cellular hypoxia of pancreatic $\beta$-cells due to high levels of oxygen consumption for insulin secretion in vitro. Journal of Biological Chemistry, 286(14), 12524-12532.

Schmidt-Kastner, R., \& Freund, T. F. (1991). Selective vulnerability of the hippocampus in brain ischemia. Neuroscience, $40(3), 599-636$.

Schulman, H., \& Greengard, P. (1978). Stimulation of brain membrane protein phosphorylation by calcium and an endogenous heat-stable protein. Nature, 271(5644), 478-479.

Scortegagna, M., Ding, K., Oktay, Y., Gaur, A., Thurmond, F., Yan, L.-J., et al. (2003). Multiple organ pathology, metabolic abnormalities and impaired homeostasis of reactive oxygen species in Epas1-/- mice. Nature Genetics, 35(4), 331-340.

Semenza, G. L. (2009). Regulation of oxygen homeostasis by hypoxia-inducible factor 1 . Physiology, 24(2), 97-106.

Semenza, G. L., Nejfelt, M. K., Chi, S. M., \& Antonarakis, S. E. (1991). Hypoxia-inducible nuclear factors bind to an enhancer element located 3 ' to the human erythropoietin gene. Proceedings of the National Academy of Sciences of the United States of America, 88(13), 5680-5684.

Semenza, G. L., \& Wang, G. L. (1992). A nuclear factor induced by hypoxia via de novo protein synthesis binds to the human erythropoietin gene enhancer at a site required for transcriptional activation. Molecular and cellular biology, 12(12), 5447-5454.

Sharp, F. R., \& Bernaudin, M. (2004). HIF1 and oxygen sensing in the brain. Nature Reviews Neuroscience, 5(6), 437-448. 
Silver, I., \& Erecińska, M. (1998). Oxygen and lon Concentrations in Normoxic and Hypoxic Brain Cells. In A. G. Hudetz \& D. F. Bruley (Eds.), Oxygen Transport to Tissue XX (pp. 7-16). Boston, MA: Springer US.

Sirén, A.-L., Fratelli, M., Brines, M., Goemans, C., Casagrande, S., Lewczuk, P., et al. (2001a). Erythropoietin prevents neuronal apoptosis after cerebral ischemia and metabolic stress. Proceedings of the National Academy of Sciences, 98(7), 4044-4049.

Sirén, A.-L., Knerlich, F., Poser, W., Gleiter, C. H., Brück, W., \& Ehrenreich, H. (2001b). Erythropoietin and erythropoietin receptor in human ischemic/hypoxic brain. Acta Neuropathologica, 101(3), 271-276.

Sirén, A.-L., Radyushkin, K., Boretius, S., Kämmer, D., Riechers, C.-C., Natt, O., et al. (2005). Global brain atrophy after unilateral parietal lesion and its prevention by erythropoietin. Brain, 129(2), 480-489.

Sommer, W. (1880). Erkrankung des Ammonshorns als aetiologisches Moment der Epilepsie. Arch. f. Psychiatr, 10, 631-675.

Spencer, J. A., Ferraro, F., Roussakis, E., Klein, A., Wu, J., Runnels, J. M., et al. (2014). Direct measurement of local oxygen concentration in the bone marrow of live animals. Nature, $508,269$.

Stroka, D. M., Burkhardt, T., Desbaillets, I., Wenger, R. H., Neil, D. A. H., Bauer, C., et al. (2001). HIF-1 is expressed in normoxic tissue and displays an organ-specific regulation under systemic hypoxia. The FASEB Journal, 15(13), 2445-2453.

Stuart, T., Butler, A., Hoffman, P., Hafemeister, C., Papalexi, E., Mauck, W. M., III, et al. (2019). Comprehensive Integration of single-cell data. Cell, 177(7), 1888-1902.e1821.

Sutter, C. H., Laughner, E., \& Semenza, G. L. (2000). Hypoxia-inducible factor $1 \alpha$ protein expression is controlled by oxygen-regulated ubiquitination that is disrupted by deletions and missense mutations. Proceedings of the National Academy of Sciences, 97(9), 4748-4753.

Suzuki, M., Kasai, K., \& Saeki, Y. (2006). Plasmid DNA Sequences Present in Conventional Herpes Simplex Virus Amplicon Vectors Cause Rapid Transgene Silencing by Forming Inactive Chromatin. Journal of Virology, 80(7), 3293-3300.

Tian, H., McKnight, S. L., \& Russell, D. W. (1997). Endothelial PAS domain protein 1 (EPAS1), a transcription factor selectively expressed in endothelial cells. Genes \& development, 11(1), 72-82.

Tsien, J. Z., Chen, D. F., Gerber, D., Tom, C., Mercer, E. H., Anderson, D. J., et al. (1996). Subregion- and Cell Type-Restricted Gene Knockout in Mouse Brain. Cell, 87(7), 13171326.

Urrutia, A. A., Afzal, A., Nelson, J., Davidoff, O., Gross, K. W., \& Haase, V. H. (2016). Prolyl-4hydroxylase 2 and 3 coregulate murine erythropoietin in brain pericytes. Blood, 128(21), 2550-2560.

van Praag, H., Kempermann, G., \& Gage, F. H. (1999). Running increases cell proliferation and neurogenesis in the adult mouse dentate gyrus. Nature Neuroscience, 2(3), 266-270.

van Praag, H., Shubert, T., Zhao, C., \& Gage, F. H. (2005). Exercise Enhances Learning and Hippocampal Neurogenesis in Aged Mice. The Journal of Neuroscience, 25(38), 86808685.

Wang, G. L., Jiang, B. H., Rue, E. A., \& Semenza, G. L. (1995a). Hypoxia-inducible factor 1 is a basic-helix-loop-helix-PAS heterodimer regulated by cellular $\mathrm{O} 2$ tension. Proceedings of the National Academy of Sciences, 92(12), 5510-5514.

Wang, G. L., \& Semenza, G. L. (1995b). Purification and Characterization of Hypoxia-inducible Factor 1. Journal of Biological Chemistry, 270(3), 1230-1237.

Wang, X., Zhang, C., Szábo, G., \& Sun, Q.-Q. (2013). Distribution of CaMKIll expression in the brain in vivo, studied by CaMKIl $\alpha$-GFP mice. Brain research, 1518, 9-25.

Waypa, G. B., Smith, K. A., \& Schumacker, P. T. (2016). O2 sensing, mitochondria and ROS signaling: The fog is lifting. Molecular aspects of medicine, 47-48, 76-89. 
Weidemann, A., \& Johnson, R. S. (2008). Biology of HIF-1 $\alpha$. Cell Death \& Differentiation, 15(4), 621-627.

Wiesener, M. S., Turley, H., Allen, W. E., Willam, C., Eckardt, K.-U., Talks, K. L., et al. (1998). Induction of Endothelial PAS Domain Protein-1 by Hypoxia: Characterization and Comparison With Hypoxia-Inducible Factor-1 $\alpha$. Blood, 92(7), 2260-2268.

Wilson, C. H., Nikolic, A., Kentish, S. J., Shalini, S., Hatzinikolas, G., Page, A. J., et al. (2016). Sexspecific alterations in glucose homeostasis and metabolic parameters during ageing of caspase-2-deficient mice. Cell Death Discovery, 2(1), 16009.

Wolf, A., Agnihotri, S., Micallef, J., Mukherjee, J., Sabha, N., Cairns, R., et al. (2011). Hexokinase 2 is a key mediator of aerobic glycolysis and promotes tumor growth in human glioblastoma multiforme. The Journal of Experimental Medicine, 208(2), 313-326.

Wood, S. M., Gleadle, J. M., Pugh, C. W., Hankinson, O., \& Ratcliffe, P. J. (1996). The Role of the Aryl Hydrocarbon Receptor Nuclear Translocator (ARNT) in Hypoxic Induction of Gene Expression: STUDIES IN ARNT-DEFICIENT CELLS. Journal of Biological Chemistry, 271(25), 15117-15123.

Wu, D., Cao, W., Xiang, D., Hu, Y.-P., Luo, B., \& Chen, P. (2019). Exercise induces tissue hypoxia and HIF-1 $\alpha$ redistribution in the small intestine. Journal of Sport and Health Science.

Wüstenberg, T., Begemann, M., Bartels, C., Gefeller, O., Stawicki, S., Hinze-Selch, D., et al. (2010). Recombinant human erythropoietin delays loss of gray matter in chronic schizophrenia. Molecular Psychiatry, 16, 26.

Yu, F., White, S. B., Zhao, Q., \& Lee, F. S. (2001). HIF-1 $\alpha$ binding to VHL is regulated by stimulussensitive proline hydroxylation. Proceedings of the National Academy of Sciences, 98(17), 9630-9635.

Yudkoff, M., Nissim, I., \& Pleasure, D. (1988). Astrocyte Metabolism of [15N]Glutamine: Implications for the Glutamine-Glutamate Cycle. Journal of Neurochemistry, 51(3), 843850.

Zampino, M., Yuzhakova, M., Hansen, J., McKinney, R. D., Goldspink, P. H., Geenen, D. L., et al. (2006). Sex-related dimorphic response of HIF-1 $\alpha$ expression in myocardial ischemia. American Journal of Physiology-Heart and Circulatory Physiology, 291(2), H957-H964. 


\section{Appendix}

\subsection{List of Abbreviations}

\begin{tabular}{|l|l|}
\hline$\%$ & Percentage \\
\hline ATP & Adenosine triphosphate \\
\hline bHLH & basic helix-loop-helix \\
\hline BOLD & Blood-oxygen-level-dependent \\
\hline CA & Cornu ammonis \\
\hline CaMKIla & Ca ${ }^{2+} /$ calmodulin-dependent protein kinase II alpha subunit \\
\hline CNS & Central Nervous System \\
\hline CO2 & Carbon dioxide \\
\hline CRW & Complex running wheel \\
\hline DG & Dentate gyrus \\
\hline EPO & Erythropoietin \\
\hline ER & Estrogen Receptor \\
\hline fMRI & Functional magnetic resonance imaging \\
\hline HIF- $\boldsymbol{\alpha}$ & Hypoxia-inducible factor $\alpha$-subunit \\
\hline HIF- $\boldsymbol{\beta}$ & Hypoxia-inducible factor $\beta$-subunit \\
\hline IF & Immunofluorescence \\
\hline LSM & Light-sheet microscopy \\
\hline NRW & Normal running wheel \\
\hline O2 & Oxygen \\
\hline ODD & Oxygen-dependent degradation domain \\
\hline PCR & Polymerase chain reaction \\
\hline PFC & Prefrontal cortex \\
\hline PHD & Prolyl-4-hydroxylase domain enzyme \\
\hline pVHL & von Hippel-Lindau tumor suppressor protein \\
\hline ROS & Reactive oxygen species \\
\hline RT & Room temperature \\
\hline SEM & Standard error of mean \\
\hline TAD & Transactivation domains \\
\hline VEGF & Vascular endothelial growth factor \\
\hline WT & Wild type \\
\hline
\end{tabular}




\subsection{Publications during PhD studies}

Butt, U.J*., Steixner-Kumar, A.A*., Depp, C*., Sun, T., Hassouna, I., Wüstefeld, L., Arinrad, S., Zillmann, M.R., Schopf, N., Garcia-Agudo, L.F., Mohrmann, L., Bode, U., Ronnenberg, A., Hindermann, M., Goebbels, S., Bonn, S., Katschinski, D.M., Miskowiak, K.W., Nave, K.A., \& Ehrenreich, H. " Hippocampal neurons respond to brain activity with functional hypoxia." Molecular Psychiatry (2021): 118.

Butt, U.J., Hassouna, I., Fernandez Garcia-Agudo, L., Steixner-Kumar, A.A., Depp, C., Barnkothe, N., Zillmann, M.R., Ronnenberg, A., Bonet, V., Goebbels, S., Nave, K.A., and Ehrenreich, H. CaMKIla Expressing Neurons to Report Activity-Related Endogenous Hypoxia upon Motor-Cognitive Challenge. International journal of molecular sciences, 2021, 22(6), p.3164.

Wakhloo, $D^{*}$., Scharkowski, $F^{*}$., Curto, $Y^{*}$., Butt, U.J., Bansal, V., SteixnerKumar, A.A., Wüstefeld, L., Rajput, A., Arinrad, S., Zillmann, M.R., Seelbach, A. Hassouna, I., Schneider, k., Ibrahim, A.Q., Werner, H.B., Martens, H., Miskowiak, K,W., Wojcik,S.M., Bonn, S., Nacher, J., Nave, K.A., \& Ehrenreich, H. "Functional hypoxia drives neuroplasticity and neurogenesis via brain erythropoietin." Nature communications 11.1 (2020): 1-12. 


\section{Co-author publication}

ARTICLE

\section{Functional hypoxia drives neuroplasticity and neurogenesis via brain erythropoietin}

Debia Wakhloo (1,11, Franziska Scharkowski 1,11, Yasmina Curto (12, 2,11, Umer Javed Butt', Vikas Bansal (1,3, Agnes A. Steixner-Kumar (1) ', Liane Wüstefeld', Ashish Rajput (1) 3, Sahab Arinrad (0) ', Matthias R. Zillmann (10) ' Anna Seelbach (1) Imam Hassouna', Katharina Schneider', Abdul Qadir Ibrahim (7) ${ }^{3}$, Hauke B. Werner (1) ${ }^{4}$, Henrik Martens (1) ${ }^{5}$, Kamilla Miskowiak $\circledast^{6}$, Sonja M. Wojcik ${ }^{7}$, Stefan Bonn ${ }^{3,8}$, Juan Nacher ${ }^{2,9,10}$, Klaus-Armin Nave (1) ${ }^{4,8 \times}$ \& Hannelore Ehrenreich (1) ${ }^{1,8 \times}$

Erythropoietin (EPO), named after its role in hematopoiesis, is also expressed in mammalian brain. In clinical settings, recombinant EPO treatment has revealed a remarkable improvement of cognition, but underlying mechanisms have remained obscure. Here, we show with a novel line of reporter mice that cognitive challenge induces local/endogenous hypoxia in hippocampal pyramidal neurons, hence enhancing expression of EPO and EPO receptor (EPOR). High-dose EPO administration, amplifying auto/paracrine EPO/EPOR signaling, prompts the emergence of new CA1 neurons and enhanced dendritic spine densities. Singlecell sequencing reveals rapid increase in newly differentiating neurons. Importantly, improved performance on complex running wheels after EPO is imitated by exposure to mild exogenous/inspiratory hypoxia. All these effects depend on neuronal expression of the Epor gene. This suggests a model of neuroplasticity in form of a fundamental regulatory circle, in which neuronal networks-challenged by cognitive tasks-drift into transient hypoxia, thereby triggering neuronal EPO/EPOR expression.

\footnotetext{
${ }^{1}$ Clinical Neuroscience, Max Planck Institute of Experimental Medicine, Göttingen, Germany. ${ }^{2}$ Neurobiology Unit, Program in Neurosciences and Interdisciolinary Research Structure for Biotechnology and Biomedicine (BIOTECMED), Universitat de València, Buriassot, Spain. ${ }^{3}$ Institute of Medical Systems Biology, Center for Molecular Neurobiology, University Clinic Hamburg-Eppendorf, Hamburg, Germany. ${ }^{4}$ Department of Neurogenetics, Max Planck Institute of Experimental Medicine, Göttingen, Germany. ${ }^{5}$ Synaptic Systems GmbH, Göttingen, Germany. ${ }^{6}$ Copenhagen Affective Disorder Research Centre Psychiatric Centre Copenhagen, Copenhagen University Hospital, Rigshospitalet, Copenhagen, Denmark. ${ }^{7}$ Department of Molecular Neurobiology, Max Planck Institute of Experimental Medicine, Göttingen, Germany. ${ }^{8}$ DFG Research Center for Nanoscale Microscopy and Molecular Physiology of the Brain (CNMPB), Göttingen, Germany. ${ }^{9} \mathrm{CIBERSAM}$ : Spanish National Network for Research in Mental Health, Valencia, Spain. ${ }^{10}$ Fundación Investigación Hospita Clínico de Valencia, INCLIVA, Valencia, Spain. "These authors contributed equally: Debia Wakhloo, Franziska Scharkowski, Yasmina Curto.

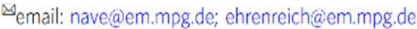


E rythropoietin (EPO) is a hypoxia-inducible growth factor in mammalian kidney, named after its role in hematopoiesis 1,2 . Unexpectedly, both EPO and its receptor (EPOR) were later detected in the brain, where they are upregulated by injury conditions. High-dose recombinant human (rh) EPO, a drug in clinical use for anemic patients, exerts neuroprotective and neuroregenerative effects that are independent of the hematocrit, which is mechanistically unexplained ${ }^{3-8}$. Moreover, rhEPO improves cognitive function and reduces gray matter loss in a range of neuropsychiatric conditions $s^{9-13}$. Even in healthy mice, rhEPO treatment improves cognition, which is associated with enhanced hippocampal long-term potentiation ${ }^{14-16}$. Surprisingly, rhEPO increases the number of mature hippocampa pyramidal neurons without underlying effect on cell proliferation or cell death ${ }^{17}$. This effect is mediated in neurons mainly by JAKSTAT, PI3K/AKT/PKB, Ras-MEK, and ERK1/2, as well as NFpathways widely comparable to the hematopoietic system ${ }^{18-20}$. This raises the question whether the expression of EPO and its receptor serves a physiological function in the nervous system, and what could be the triggering factors of EPO expression under physiological conditions.

\section{Results}

Generation of pyramidal neurons in adult mice and amplification by rhEPO. First, we developed a method to directly labe and quantify newly generated neurons in the hippocampal cornu ammonis (CA) field of adult mice. This was possible by permanently labeling all mature pyramidal neurons present at P27 using a tamoxifen-inducible reporter gene in NexCreERT2::R26R-tdT mice (Fig. la, b) ${ }^{21}$. Thus, all neurons differentiating and maturing after termination of the tamoxifen-induced Cre recombination lack tdTomato, but can be positively identified by Ctip2, a specific marker of pyramidal neurons, thereby revealing adult 'neurogenesis' independent of DNA synthesis.

Immediately after tamoxifen induction, at P28, the number of such unlabeled (tdTomato ${ }^{-}$, Ctip $2^{+}$) pyramidal neurons, quantified for control purposes, was $<3 \%$ of all Ctip $2^{+}$cells. When rhEPO treatment was initiated at P28 as described ${ }^{17}$, and mice were analyzed at P55, we found a considerable number of newly differentiated (tdTomato ${ }^{-} / \mathrm{Ctip}^{+}$) neurons in CAl, withou evidence by 5 -Ethynyl-2'-deoxyuridine (EdU) incorporation of proliferating precursors, as we reported previously ${ }^{17}$. Upon rhEPO treatment, the number of pyramidal cells was higher compared to placebo, but even untreated mice revealed a remarkable increase in new neurons (Fig. 1c)

Strikingly, when we applied the same rhEPO treatment to older mice (age 3 months), we detected a comparable effect on pyramidal neuron numbers (Fig. 1d). Thus, there is a substantia generation of pyramidal neurons from pre-existing (nonproliferating) precursors also in adulthood, suggesting a previously overlooked aspect of adult neurogenesis, discovered by serendipity in rhEPO-treated mice.

EPO increases dendritic spine density. Using Thyl-EYFP transgenic mice ${ }^{22}$, we noted that EPO treatment also enhanced the dendritic spine density in pyramidal neurons at both $\mathrm{P} 55$ and 4 months (Fig. 1e-i). The analysis of spine morphology (Fig. 1j-l) showed an increase in stubby immature spines and a trend toward more mature mushroom spines ${ }^{23}$. Reflecting the increased pyramidal neuron number, we also noted a higher density of Map2 immunoreactive primary dendrites in the stratum radiatum (Fig. $1 \mathrm{~m}-\mathrm{o}$ )

Immediate differentiation response of neuronal precursors to rhEPO. We employed single-cell transcriptome analysis (scRNA- seq) to explore the immediate response of cells in CAl to rhEPO, detectable $6 \mathrm{~h}$ after a single intraperitoneal (i.p.) injection of $5000 \mathrm{U}$ rhEPO/kg (Fig. 2, Supplementary Figs. 1-3, Source Data File). This revealed alterations in the cell cluster composition as defined by gene expression profiles ${ }^{24}$, with more cells in the immature glutamatergic differentiation cluster after rhEPO (Fig. 2a-d). Markers that characterize this cluster include doublecortin $(D c x)$, transducin-like enhancer family member4 (Tle4), T-box brainl (Tbr1), rhabdomyosarcoma 2-associated transcrip (Rmst), SRY-Box5 (Sox5), DNA-binding protein SATB1 (Satb1) ELAV-like neuron-specific RNA-binding protein4 (Elavl4), fucosyltransferase9 (Fut9), CUGBP Elav-like family member4 (Celf4), and heparan sulfate-glucosamine 3-sulfotransferase4 (Hs3st4). For two of them, Tle4 and Tbrl, in situ validation of protein expression by immunohistochemistry (IHC), predominantly in $\mathrm{Ctip}^{+}$neurons (confirmed by double labeling) at $\mathrm{P} 29$, i.e., $24 \mathrm{~h}$ after a single injection of EPO/placebo, exemplifies the distinct EPO effect (Fig. 2e). Note that Tbrl $^{+}$neurons upon EPO are rapidly and strongly detected in CA1 at P29, but only rarely anymore at $\mathrm{P} 55$. Tbr1 ${ }^{+}$cells in dentate gyrus serve as positive control for both time points (Supplementary Fig. 3e) Other neuronal clusters and non-neuronal cell types were not obviously different between treatment groups at $6 \mathrm{~h}$ (Supplementary Fig. 3b)

In order to further investigate the EPO-induced effects on cell differentiation, we performed a trajectory (pseudotime) analysis on cells in the immature glutamatergic cluster and its neighboring cluster, mature glutamatergic1, in Monocle ${ }^{25}$; Fig. 2f; list of differentially expressed genes between these two clusters given in Source Data File). The analysis confirmed the immature identity of cells in the immature glutamatergic cluster, reflected by homogeneously low pseudotime of almost all cells in this cluster, which was significantly lower as compared to cells in the mature glutamatergic1 cluster (Fig. 2g). After post hoc exploratory removal of immature cells with high pseudotime $(>15, n=10)$ the increase in immature cells upon EPO still remained significant $(p=0.039$; Fisher's exact test, one sided). Even if the immature cells with high pseudotime $(>15)$ were reassigned to the mature glutamatergic cluster, a significance level of $p=0.041$ was obtained. Importantly, we observed a generally lower pseudotime upon EPO, independent of cluster assignment, further supporting our observation of an increase in immature cells and excluding a mere 'clustering artifact' (Fig. 2h). Also, reclustering in Monocle 2 of cells from the two clusters revealed a robust detection of the immature cluster across methods (Seurat and Monocle2; Supplementary Fig. 3a) and a robustly increased number of immature cells upon EPO as compared to placebo treatment, independent of the analysis platform $(p=0.0008)$. For further validation of the immature identity of these cells see Supplementary Fig. 3c, d.

Together, these data provide supportive evidence of an immediate effect of EPO on pre-existing precursors to instantaneously initiate neuronal differentiation, thereby rapidly increasing the numbers of cells in the immature glutamatergic differentiating cluster. Expectedly, EPO and EPOR were not detected by scRNA-seq due to their very low expression level, a known drop-out effect in scRNA-seq data.

Elevated expression of EPO and EPOR by pyramidal neurons upon CRW. Searching for the physiological significance of these responses, we explored whether EPO and EPOR are endogenously expressed by pyramidal neurons. Using a highly sensitive in situ hybridization (ISH) method, capable of detecting even single mRNA molecules, we identified both EPO and EPOR transcripts in pyramidal neurons of the hippocampal CAl region (Fig. 3a-d). The overall mRNA abundance in the hippocampus 


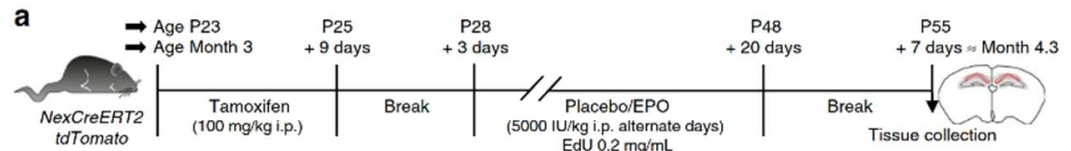

b
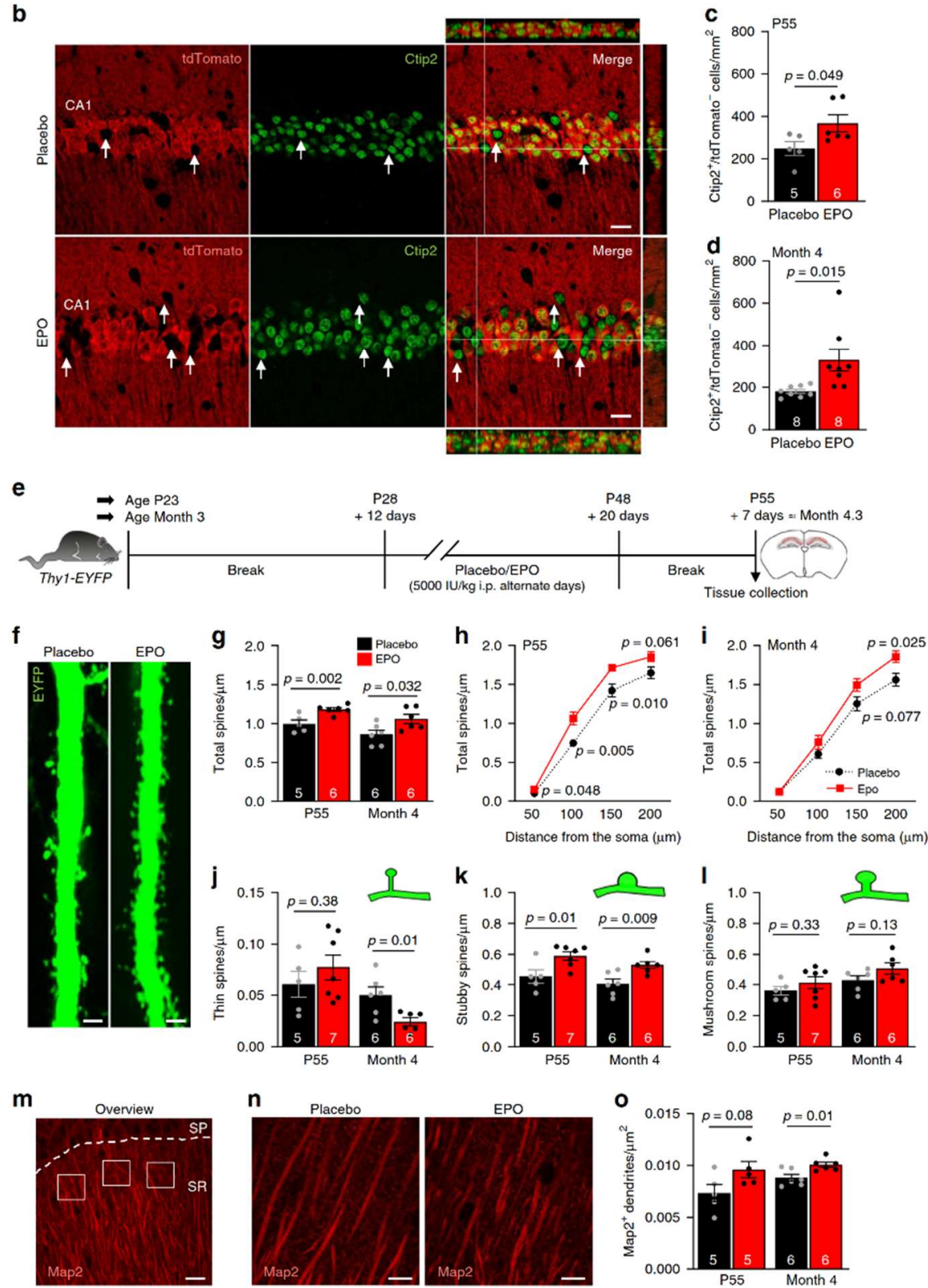

was higher than in cortex and other brain regions. Interestingly, when mice were placed into cages with complex running wheels $(\mathrm{CRW})^{26}$, a cognitive challenge that stimulates intricate motor learning and coordination, only 5-9 h of running in the dark phase were sufficient to upregulate the expression of EPO and EPOR transcripts in pyramidal neurons (Fig. 3a-d).

Permanent labeling of hypoxic neurons in CA1 by CRW exposure. We therefore wondered which mechanism would lead to the swift upregulation of EPO in pyramidal neurons upon cognitive challenge. In the kidney, it is hypoxia and hypoxiainducible factors (Hif), which are well known to induce EPO expression ${ }^{1,2,7,27}$. We therefore hypothesized that cognitive 
Fig. 1 EPO increases number of pyramidal neurons and spine density in the CA1 region of the hippocampus. a Experimental design to determine the effect of EPO on neuron numbers: two cohorts of NexCreERT2::tdTomato mice (starting at age P23 or month 3) were administered tamoxifen (at P23: $5 \times$ i.p.; at month 3: $10 \times$ i.p.), followed by placebo or EPO (5000IU/kg; $11 \times$ i.p.) on alternate days for 3 weeks. b Representative images of tdTomato (red) and Ctip2 (green; excitatory neuronal marker) staining in CA1 of placebo or EPO-treated mice. White arrows indicate newly generated neurons. c. d Quantification of newly generated neurons (Ctip2 ${ }^{+} /$tdTomato $^{-}$) in CA1 of P55 c and -4-month-old mice $\mathbf{d}$ treated with placebo or EPO. Data from $n=5 / 8$ (placebo; $1 \mathrm{c} / \mathrm{d}$ ) and $n=6 / 8(E P O ; 1 c / d)$ mice. e Experimental design to determine the effect of EPO on dendritic spine number and morphology: Thy1-EYFP mice (starting at age P28 or month 3) were treated with placebo or EPO (11x i.p.) on alternate days, for 3 weeks. $\mathbf{f}$ Representative images of EYFP (green) expression in principal apical dendrites in CA1 of placebo or EPO mice. g-i For P55 and $\sim 4$-month-old mice, total number of spines (per $\mu$ m) are given as bar graphs $\mathbf{g}$ and, additionally, as line graphs $\mathbf{h}$-i presenting their distance from the soma. Data from $n=5 / 6$ (placebo; P55/4 months) and $n=6$ (EPO) mice ( 6 cells/mouse analyzed). $\mathbf{j}-\mathbf{I}$ Based on the morphology (illustrated as green insets), the number of thin $\mathbf{j}$, stubby $\mathbf{k}$, and mushroom I spines (per $\mu \mathrm{m}$ ) were sub-quantified. $\mathbf{m}$ Representative image of the quantification method employed for determining the number of dendrites using dendritic marker Map2 (red) in CA1 stratum radiatum (SR); stratum pyramidale (SP). White boxes denote areas of measurements. $\mathbf{n}$ Representative images of Map2 (red) staining in 4-month-old mice treated with placebo or EPO. o Quantification of Map2 $2^{+}$dendrites in P55 and 4-month-old mice treated with placebo or EPO. Data from $n=5 / 6$ (placebo; P55/4 months) and $n=5 / 6$ (EPO; P55/4 months) mice. Within bars, mouse $n$ numbers indicated; mean \pm SEM presented; two-tailed Student's t-test; scale bars: $\mathbf{b} 50 \mu \mathrm{m} ; \mathbf{f} 10 \mu \mathrm{m} ; \mathbf{m} 20 \mu \mathrm{m}$; and $\mathbf{n} 10 \mu \mathrm{m}$. Source data underlying graphs c, d, g-I, and $\mathbf{0}$ are provided as a Source Data File.

challenge, such as learning of complex motor tasks, is associated with neuronal activity that requires more oxygen than present in the normal steady state. This could induce a mild (transient) endogenous hypoxia as a potential signal underlying upregulated expression of EPO in highly active pyramidal neurons. To tes this hypothesis, we asked whether 'functional hypoxia' can be detected in the brain after cognitive challenge, such as complex wheel running. To this end, we created a novel transgenic mouse for the ubiquitous expression of a chimeric fusion protein between the tamoxifen-inducible CreERT2 recombinase and the oxygen-dependent degradation (ODD) domain of Hif- $1 a^{28}$. In mice bearing this CreERT2-ODD transgene, driven by the CAG promoter, 'fate mapping' and identification of (transiently) hypoxic cells is possible with the tdTomato reporter (CAG CreERT2-ODD::R26R-tdTomato). Indeed, exposing these mice after tamoxifen injection to CRW over one night, we determined a distinctly increased number of 'ODD-labeled' pyramidal neurons in the $\mathrm{CAl}$ region when compared to non-runners. This indicates that a new challenging task has led to more (transiently) hypoxic neurons, which became permanently labeled. Paralle quantification of cFos expression as neuronal activity marker ${ }^{29}$ revealed a tendency of increased cFos labeling upon CRW (Fig. $3 \mathrm{e}-\mathrm{h}$ ), but no double labeling with ODD-tdTomato. The latter likely occurs too late in a respective cell, i.e., when its immediate early gene (cFos) expression has already disappeared. In contrast, after only $4 \mathrm{~h}$ of CRW, cFos labeling was obvious, but no ODD-tdTomato labeling yet detected $\left(\mathrm{CFos}^{+}\right.$cells: nonrunners $40.1 \pm 20.8, n=3$; CRW $143.3 \pm 24.4, n=7 ; p=0.034$ mean \pm SEM). Additional confirmation of hypoxic cell labeling by ODD-tdTomato was obtained using pimonidazole, resulting in findings similar to a previous report on heart ${ }^{28}$ (Supplementary Fig. 4).

EPO treatment strongly increases motor learning and endurance. To further address the potential of rhEPO to lastingly improve cognitive performance, we treated juvenile mice with our protocol that results in $\sim 20 \%$ more pyramidal neurons (starting at $\mathrm{P} 28$, i.p. injection of $\mathrm{rhEPO} /$ placebo every other day for 3 weeks, followed by a 1-week break). Afterward, the running-naive mice were transferred to cages containing CRW. During the initia learning phase, a rise in performance was observed, clearly dissociating EPO from placebo-treated mice after just a few hours, followed by enhanced endurance over the whole night.

Deletion of neuronal EPOR attenuates EPO-/hypoxia-induced motor learning. Theoretically, the EPO-induced increase in hematocrit could account for this enhanced performance. To rule this out and to explore whether pyramidal EPOR is mediating this effect, we deleted the Epor gene from pyramidal neurons (NexCre::EpoRfl/fl; Supplementary Fig. 5). More details on the generation of this conditional Epor allele, its recombination with different Cre-lines, and a comprehensive phenotype analysis will be described elsewhere (manuscript in preparation). As delineated in Fig. $4 \mathbf{a}-\mathbf{c}$, the remarkable rise in performance of wildtype (WT) mice following EPO treatment reported above was well replicated, whereas EPOR-cKO mice failed to show any noticeable effect of EPO treatment on CRW performance (i.e., complex motor learning and endurance). Placebo-treated EPORcKO did not appreciably differ from WT mice (Fig. 4c, inset). The behavioral phenotype of EPOR-cKO mice strongly suggested that also neuronal complexity, i.e., dendritic spine density of pyramidal neurons, would be affected by EPOR deletion. Indeed, Golgi staining revealed that lack of EPOR in pyramidal neurons prevented the EPO-induced increase in dendritic spines (Fig. 4d, e).

Mild inspiratory hypoxia acts synergistically with cognitive challenge. Since learning to run on CRW leads to endogenous ('functional') hypoxia in pyramidal neurons, as demonstrated by ODD labeling, and since pyramidal EPO and EPOR mRNAs are upregulated by functional hypoxia, we wondered whether CRW exposure for several weeks would (similar to rhEPO treatment) also lead to more pyramidal neurons. Moreover, would a sustained exposure of mice to a mild exogenous (inspiratory) hypoxia $\left(12 \% \mathrm{O}_{2}\right)$, when coinciding with running activity, result in similar or even synergistic effects, i.e., better learning performance and more neurons in the stratum pyramidale? In the respective large-scale, multi-arm experiment over 3 weeks, we indeed detected improved motor learning and endurance on the CRW of mice under mild hypoxia when compared to normoxia (Fig. 4f-k). To unequivocally demonstrate that the neuronal EPO/ EPOR system is responsible for the observed effects, we employed again our mice with targeted deletion of the Epor gene in pyramidal neurons. In fact, lack of EPOR in pyramidal neurons resulted in a distinct reduction of the learning curve slope over time, both under normoxic and hypoxic conditions, when compared to the respective WT controls. Also, non-running EPORcKO mice have already less pyramidal neurons independent of hypoxia (normoxia: $7834.07 \pm 223.18$ in cKO versus $9377.48 \pm$ 864.48 in WT; hypoxia: $8431.51 \pm 320.40$ in cKO versus 10730.78 \pm 497.00 in WT; overall genotype effect $p=0.0012$ ) and fail to show the increase in pyramidal neurons found in WT mice upon running with or without hypoxia (Fig. $4 \mathrm{f}-\mathrm{k}$ ). 
a

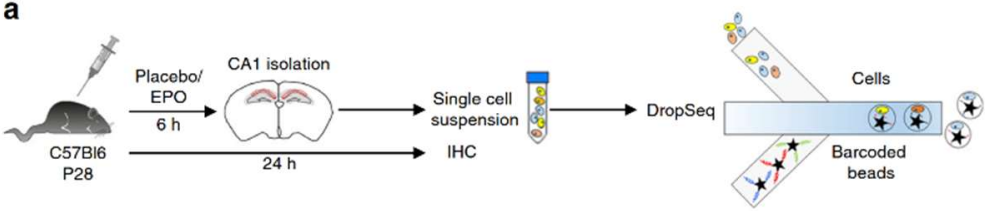

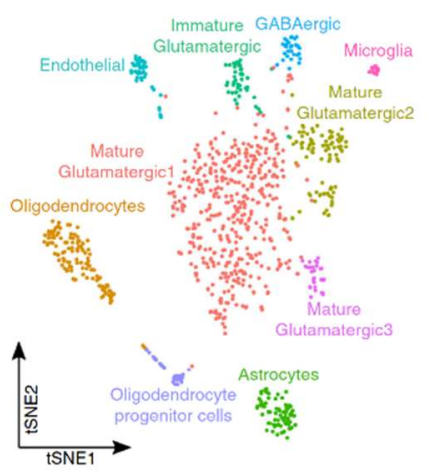

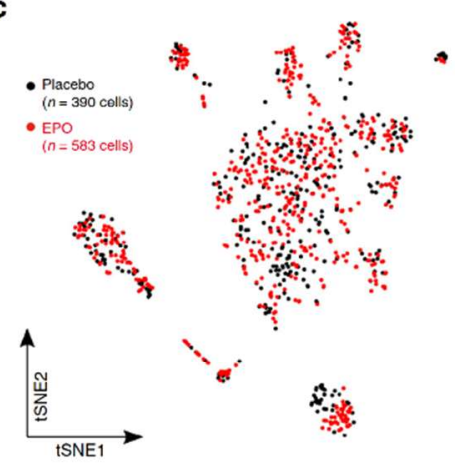

d

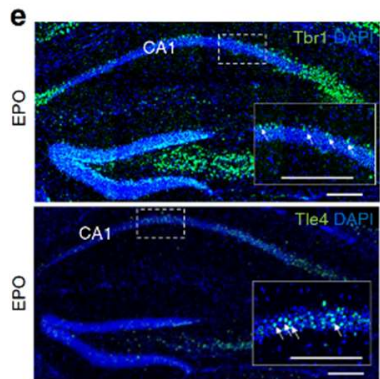

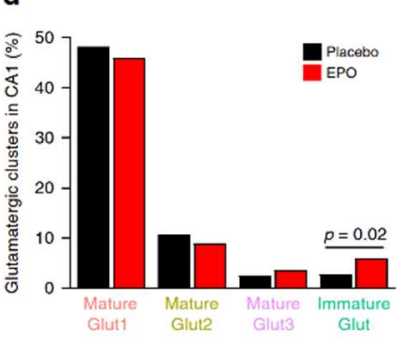

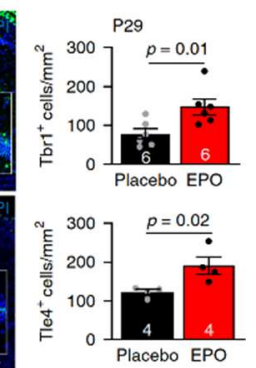

g

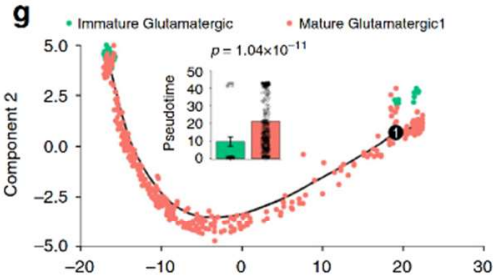

f

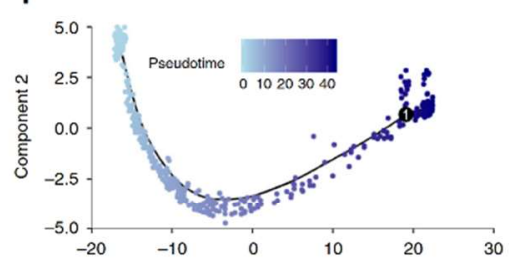

h

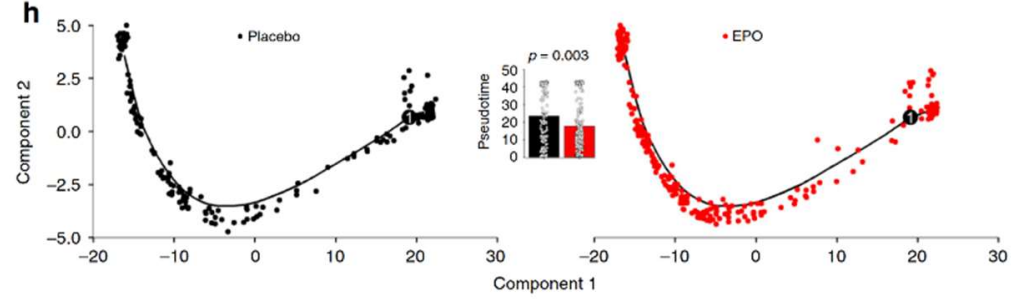

Expression of cFos marks newly differentiated neurons. Under both normoxic and hypoxic conditions, we counted more recently differentiated (EdU negative) pyramidal neurons in runners than in non-runners in the hippocampal CA1 field. We thus wondered whether the newly differentiated pyramidal neurons, generated in mice placed into CRW cages, are functional. To address this question, we challenged animals for 3 weeks in CRW (with or without mild inspiratory hypoxia) but moved them first back to regular cages for 1 week, in analogy to the rhEPO treatment scheme. Then, we re-exposed the mice to CRW for only $4 \mathrm{~h}$ at the beginning of their dark phase. Immediately thereafter, they were sacrificed and brains processed for IHC. As readout of neuronal activity and functional integration, cFos expression ${ }^{29}$ was used again and found to be enhanced following hypoxia, and in 
Fig. 2 Transcriptome analysis (scRNA-seq) demonstrates an immediate increase in neurodifferentiation upon EPO application. a Experimental design of WT C57B|6 mice ( $n=3$ per group), treated at P28 with a single i.p. injection of placebo or EPO ( $5000 \mathrm{IU} / \mathrm{kg}$ ), followed by isolation of the CA1 region after $6 \mathrm{~h}$ and processing for DropSeq analysis (processing of biological replicates performed separately, followed by pooling for graphical presentation and final analysis), as well as after $24 \mathrm{~h}$ for immunohistochemistry (figure created by Debia Wakhloo). b Visualization of hippocampal CA1 cell clusters using $t$-distributed stochastic neighbor embedding (tSNE). Each color represents a cluster of specific cells, characterized by a defined gene expression profile. c Individual cells derived from either EPO (red) or placebo (black)-treated mice are denoted. d Percentage of cells in the respective glutamatergic clusters per treatment condition (EPO: $n=583$, placebo: $n=390$ ); two-tailed Fisher's exact test. e Representative images and quantification of Tbr1 and Tle4 staining (immature neuronal markers) in CA1 of mice at P29, i.e., $24 \mathrm{~h}$ after a single injection of EPO. Within bars, mouse n numbers indicated; mean \pm SEM presented; two-tailed student's t-test. $\mathbf{f}$ Trajectory analysis in Monocle2 of cells in the 'Mature Glutamatergicl' and the 'Immature Glutamatergic' cluster colored by pseudotime (the darker the more mature). $\mathbf{g}$ Trajectory colored by cell identity. Bar graph indicates average pseudotime of the respective clusters; mean \pm SEM; two-tailed Mann-Whitney $U$ test. h Trajectory colored and split by placebo (black; left) versus EPO treatment (red; right). Bar graph shows average pseudotime of cells in the respective treatment groups; two-tailed Mann-Whitney U test; mean \pm SEM; see also Supplementary Figs. 1-3. Source data underlying graph e are provided as a Source Data File.

particular after running. Unexpectedly, cFos was mainly detected in the recently differentiated neurons, whereas the fraction of pre-existing neurons expressing cFos was small (Fig. 41-p).

\section{Discussion}

Taken together (Fig. 5), we have discovered that EPO - experimentally and clinically shown to improve cognition ${ }^{9-17}$ - stimulates a previously unrecognized mechanism of cellular neuroplasticity. Specifically, rhEPO (when peripherally administered) enters the brain, where it mimics the effects of endogenous neuronal EPO/ EPOR signaling, stimulating dendritic spine formation on mature cells, and the differentiation of new pyramidal neurons from preexisting (non-proliferating) precursors, the identity of which is currently unknown.

The EPO-responsive cells undergoing adult neurogenesis likely have no defining single genetic marker, suitable for CreER-based lineage tracing (unlike the $\mathrm{EPO}-$ responsive $\mathrm{OPC}^{17}$ ). Not even direct transdifferentiation of endothelial and astroglial cells or dedifferentiation of neurons can be completely excluded at this point. Jak2-Stat5 signaling, however, a pathway important for neuronal EPOR effects ${ }^{18,20}$, is known in various tissues to induce terminal differentiation, not dedifferentiation.

We hypothesize that the entire precursor cell lineage that is ready to differentiate toward pyramidal neurons in adult mice remains 'in flow'. Similar to adaptive metabolic pathways, in which the 'flux' of metabolites matters more than the different steady-state concentrations, also in the proposed neuronal lineage progression, the EPO-responsive progenitor cells may never constitute abundant clusters in a cross-sectional steady-state analysis. This is remarkably similar to the effects of EPO on multiple precursors at different stages in the hematopoietic system $1,30-32$

Pyramidal neurons, when challenged by novel tasks, undergo mild hypoxia, as detected here by 'ODD labeling', using hypoxia reporter mice. This activity-induced 'functional hypoxia' stimulates endogenous EPO expression by pyramidal neurons. In parallel, we find also EPOR expression enhanced, caused either directly by hypoxia ${ }^{33}$ or by EPO in an auto/paracrine manner ${ }^{34}$ When EPOR expression is deleted from pyramidal neurons, the endogenous EPO/EPOR system can no longer contribute to adaptive increase in performance.

Contrary to popular viewpoints that hypoxia is detrimental, recent reviews also consider beneficial effects and protection against cognitive dysfunction ${ }^{35,36}$. Our data using CRW indicate that hypoxia can act as driving force of long-lasting cellular neuroplasticity, complementing synaptic plasticity. Neuronally produced EPO/EPOR likely constitutes an auto/paracrine mechanism, possibly in concert with other hypoxia-inducible genes, like vascular endothelial growth factor, which has previously also been reported to enhance cognition ${ }^{37}$. We suggest a model of an intrinsic EPO/EPOR-mediated mechanism of cellular neuroplasticity (Fig. 5), in form of a fundamental regulatory circle, which can explain the remarkable procognitive effects of rhEPO, consistently found in humans and rodents, health and disease ${ }^{9,10,12,14-16,38}$

A recent study documented EPO mRNA in different neural cell types in the embryonic brain (mouse organogenesis cell atlas$\mathrm{MOCA}^{39}$ ). The extraordinarily low expression of EPO and EPOR in the adult brain may explain why this system has previously not been rigorously studied. So far, we have focused on the hippocampal CAl region, where we had obtained first evidence of substantially increased neuron numbers after rhEPO treatment ${ }^{17}$. CA1 is involved in temporal pattern association and different facets of memory formation and consolidation ${ }^{40}$. Future studies will have to address $\mathrm{EPO} / \mathrm{EPOR}$ functions in other CNS regions, including cortical areas known to be also engaged in CRW learning ${ }^{41}$, as well as neuron-glial interactions.

'Adult neurogenesis' from pre-existing precursors without proliferation extends the concept and pivotal work from many groups who discovered adult neurogenesis in distinct brain regions based on the selective labeling of proliferating cells in $\mathrm{S}$-phase ${ }^{42-48}$. More complex than oligodendrogenesis from preexisting precursors ${ }^{49}$, multiple neuronal progenitors may be relevant for maintaining neuron numbers in steady state, for adjustment to demand, and for rapid regenerative processes. The genetic approach of EPOR deletion in pyramidal neurons, together with the discovery of 'functional hypoxia' in the behaving brain, allowed us to propose a novel working model (Fig. 5). This model may add to our present concepts of neuroplasticity and adult neurogenesis.

\section{Methods}

Experimental models and mouse genetics. All experiments were approved by and conducted in accordance with the regulations of the local Animal Care and Use Committee (Niedersächsisches Landesamt für Verbraucherschutz und Lebensmittelsicherheit, LAVES). WT C57BL/6N (Charles River), NexCreERT2 $2^{21}, R 26 R$ tdTomato ${ }^{50}$, Thy1-YFPH (Jackson Laboratory, 003782), CACCreERT2-ODD ${ }^{28}$, $\mathrm{NexCre}{ }^{51}$, and EPOR-floxed (EPOR $\left.{ }^{\mathrm{fl} / \mathrm{fl}}\right)$ mice were used for the experiments. Juvenile (P23) and adult (3 months old) mice were used in this study. For genetic labeling of projection neurons, NexCreERT2 mice were bred with Rosa26 floxed stop tdTomato (R26R-tdTomato) reporter mice to generate NexCreERT2:R26R$t d T o m a t o$ mice. For labeling cells undergoing hypoxia, CAGCreERT2-ODD mice were employed ${ }^{28}$. Briefly, a fusion protein consisting of the ODD domain of Hif-1 $\alpha$ and a ubiquitous CAGCreERT2 is expressed upon tamoxifen induction. CAGCreERT2-ODD were bred with $R 26 R$-tdTomato to generate CAGCreERT2 $O D D$ : dT ODD:IdTomato reporter mice. Upon tamoxifen induction, cells are irreversibly labeled with tdTomato containing stabilized Hif-la. NexCre::Eporly? mice we generated to specifically delete Epor in projection neurons. For the generation of Eportl/t mice, embryonic stem cells (ES) harboring an engineered allele (Epormla(KOMP)Wisi) of the Epor gene were acquired from the Knockout Mouse Project (KOMP, University of California, Davis CA 95618, USA). ES cells were microinjected into blastocysts derived from C57BL/6N mice and the embryos wer transferred to pseudopregnant foster mothers, yielding chimeric males. For ES clone EPD0316_5_A03, germline transmission was achieved upon breeding with 
a

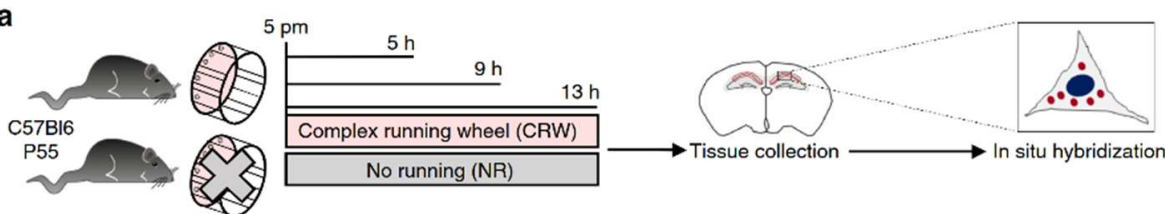

b

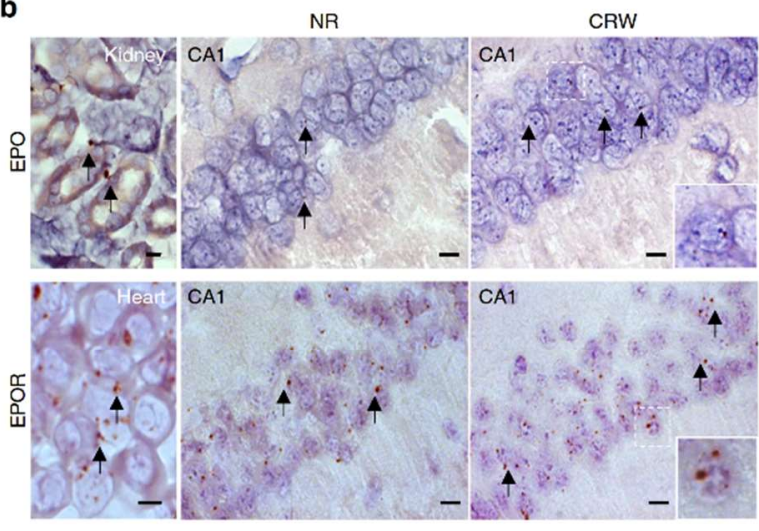

c
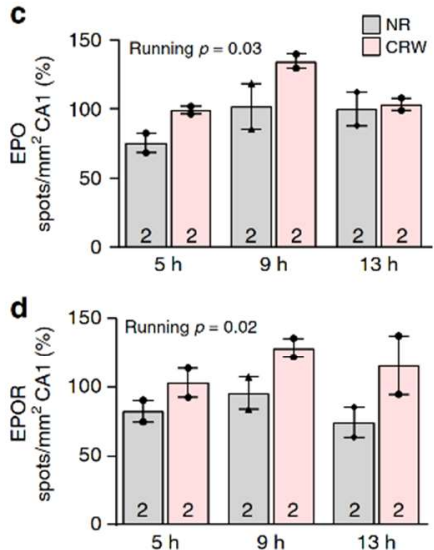

e

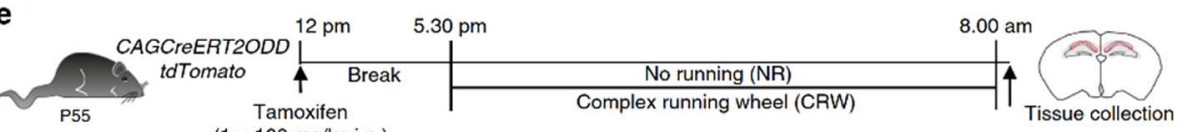
$(1 \times 100 \mathrm{mg} / \mathrm{kg}$ i.p. $)$
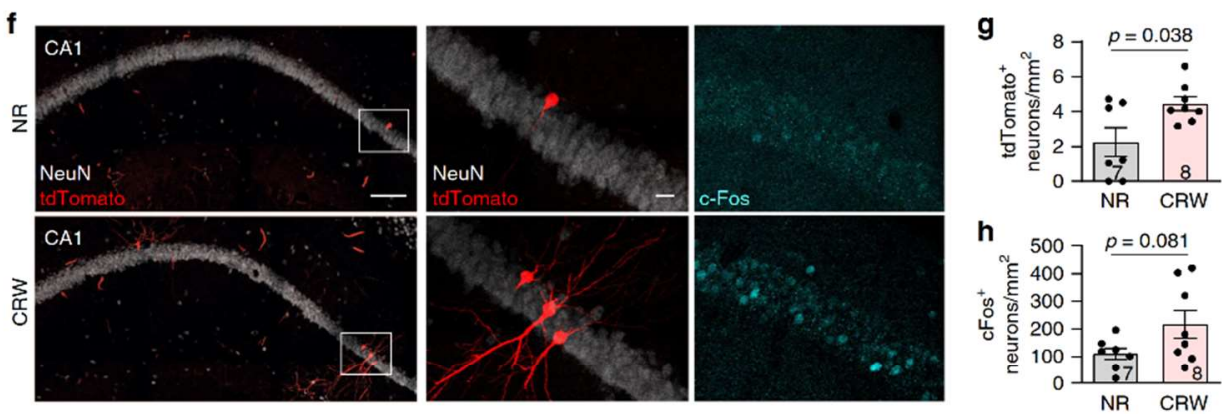

Fig. 3 Expression of the EPO/EPOR system in pyramidal neurons shown by ISH and its upregulation by functional hypoxia. a Experimental design of WT C57BI6 mice exposed to no running (NR) or voluntary running on CRW at P55 for 5, 9, or $13 \mathrm{~h}$. ISH was performed on CA1 regions (figure created by Debia Wakhloo). b Representative ISH images of CA1 regions of non-runners (NR) and runners (CRW), demonstrating EPO and EPOR mRNA expression in pyramidal neurons (brown spots). As positive controls for EPO/EPOR mRNA expression, sections of kidney (P55) and heart (E14.5) from WT C57BI6 mice are presented. $\mathbf{c}$, d Quantification of total number of EPO or EPOR mRNA spots in CA1 of non-runners (NR) and runners (CRW), shown at 5, 9, and $13 \mathrm{~h}$; mean and range shown; two-way ANOVA; $n$ numbers indicated within bars; scale bars in b: $10 \mu \mathrm{m}$; for heart $5 \mu \mathrm{m}$. experimental design to determine the effect of running on the induction of functional hypoxia in the CA1 region. CAGCreERT2-ODD::tdTomato mice were administered tamoxifen at P55 of age and exposed to either no running (NR) or overnight voluntary CRW. $\mathbf{f}$ Representative images of neuronal marker NeuN (white), tdTomato (red; labeling hypoxia), and cFos (cyan) in non-runner (NR) and runner mice (CRW); scale bars $100 \mu \mathrm{m}$ (left); $10 \mu \mathrm{m}$ for magnifications. $\mathbf{g}$, $\mathbf{h}$ Quantification of hypoxic neurons (tdTomato ${ }^{+}$) and active neurons ( $\mathrm{cFos}^{+}$) in CA1 after CRW versus NR overnight ( $12 \mathrm{~h}$ ); mean \pm SEM; two-tailed Student's t-test; data from $n=7$ (non-runners, NR) and $n=8$ (CRW) mice. Source data underlying graphs $\mathbf{c}, \mathbf{d}, \mathbf{g}$, and $\mathbf{h}$ are provided as a Source Data File.

C57BL/6N females, generating mice harboring the Epor ${ }^{\prime m I a(K O M P) W i s i}$ allele (termed EporlacZ-neo $)$. The lacZ-neo cassette was excised in vivo upon interbreeding with mice

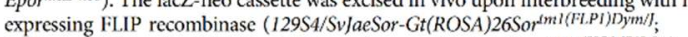

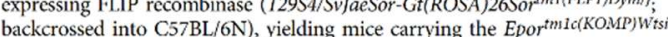
allele (termed Epor $r^{f o x}$ ). To recombine the Epor gene specifically in projection neurons, exons $3-6$ were excised in vivo upon appropriate interbreedings of EportmIc(KOMP)Wtsi mice with mice expressing Cre recombinase under control of the Nexl/Neurod6 promoter $^{51}$, generating mice carrying the Epor ${ }^{2 m l d(K O M P) W l s i}$ allele (NexCre::Epor fllth $_{\text {mice). }}$.

All mice were housed in a temperature controlled environment $\left(21 \pm 2^{\circ} \mathrm{C}\right)$ on a $12 \mathrm{~h}$ light-dark cycle with food and water available ad libitum. Male mice of the same age were used for the experiments, unless stated otherwise. All animals were genotyped before the start of each experiment. Detailed PCR protocols are available on request. 
a

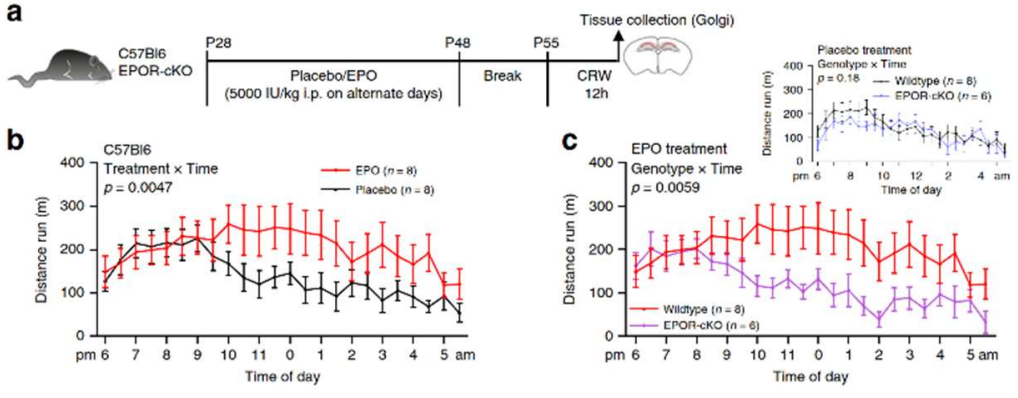

d
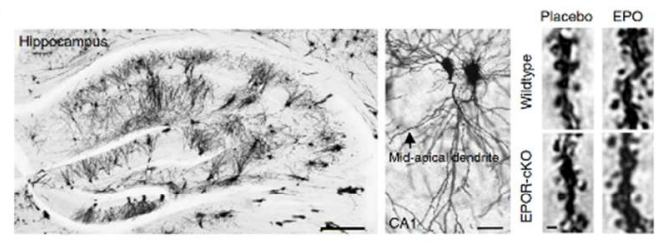

e

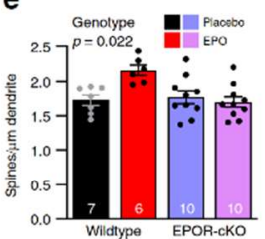

f
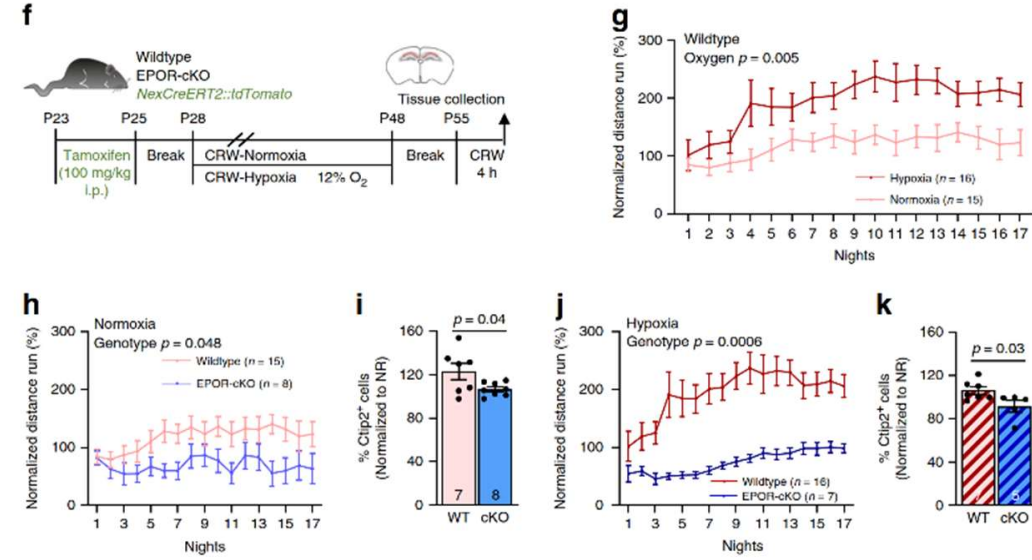

k
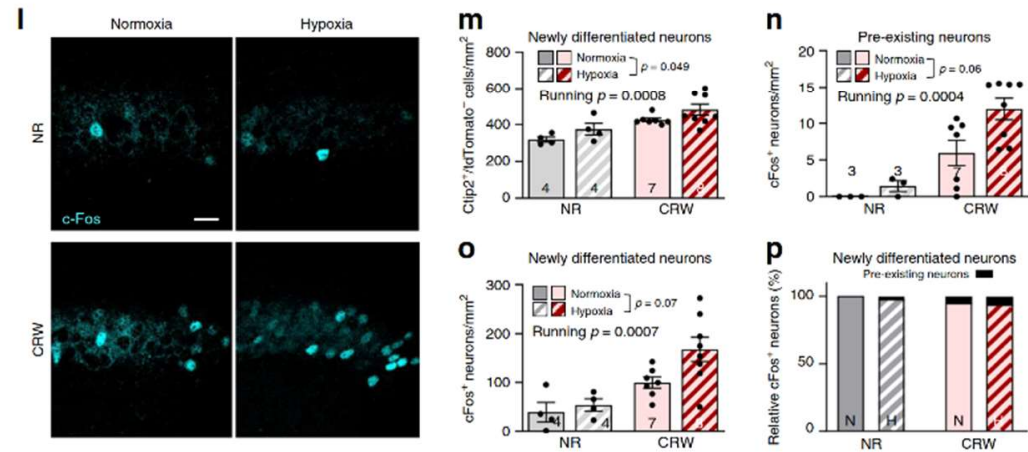

Mouse treatment. Tamoxifen: Tamoxifen solution $(10 \mathrm{mg} / \mathrm{ml})$ was freshly prepared by dissolving tamoxifen freebase (Sigma) in corn oil (Sigma) at room temperature (RT) for 45 min. Postnatal CreERT2 activity in NexCreERT2 mice was induced by a total of five i.p. injection of $100 \mathrm{mg} / \mathrm{kg}$ tamoxifen over the course of 3 days in juvenile mice. For CreERT2 induction in adult NexCreERT2 mice, a total of ten i.p. injections of $100 \mathrm{mg} / \mathrm{kg}$ tamoxifen were administered over the course of ten consecutive days. For the desired induction of CreERT2 in CAGCreERT2-ODD mice, a single i.p. injection of $100 \mathrm{mg} / \mathrm{kg}$ tamoxifen was sufficient.

EPO: Male mice were i.p. injected with 5000IU/kg rhEPO (NeoRecormon, Roche) or placebo (solvent solution, $0.01 \mathrm{ml} / \mathrm{g}$ ). At $48 \mathrm{~h}$ after the last tamoxifen injection, EPO/placebo treatment was initiated in P28 or 3-month-old NexCreERT2, Thy1-YFPH and also in P28 old WT and NexCre::EPOR R//n mice, and carried out every other day for 3 weeks. For DropSeq analysis, EPO was administered once followed by tissue collection $6 \mathrm{~h}$ later. Additionally, for labeling of proliferating cells, NexCreERT2 mice obtained EdU $(0.2 \mathrm{mg} / \mathrm{ml}$; ThermoFisher) via drinking water (exchanged on all alternate days). 
Fig. 4 Targeted deletion of EPOR in pyramidal neurons attenuates EPO/hypoxia-induced motor learning. a Experimental design. b Total distance run by placebo (black) and EPO (red) treated WT mice. c Total distance run by WT (red) and EPOR-cKO mice (purple) following EPO; insert shows for comparison total distance run by WT (black) and EPOR-cKO mice (blue) upon placebo. d Representative images of hippocampal section processed with GolgiStain ${ }^{\top M}$ Kit (scale bar: $100 \mu \mathrm{m}$ ), CA1 pyramidal neurons (scale bar: $20 \mu \mathrm{m}$ ), and mid-apical dendrites (scale bar: $1 \mu \mathrm{m}$ ). e Quantification of dendritic spines in WT and EPOR-cKO mice treated with placebo or EPO. $f$ Experimental design. WT and EPOR-cKO mice were exposed to NR or CRW under normoxia $\left(21 \% \mathrm{O}_{2}\right)$ or inspiratory hypoxia $\left(12 \% \mathrm{O}_{2}\right)$, starting at P28 for 3 weeks. After 1 week of break, CRW mice were again exposed to voluntary CRW for $4 \mathrm{~h}$ (cFos activation) before being sacrificed at P55 (results in $\mathbf{g - k}$ ); NexCreERT2::tdTomato mice were injected with tamoxifen (5× i.p.-starting at P23) and exposed to same experimental paradigm (results in I-p). $\mathbf{g}$ CRW curves over 17 nights (normalized to mean distance over first three nights) of WT mice exposed to continuous inspiratory normoxia versus hypoxia. h-k Effect of EPOR deletion in pyramidal neurons: $\mathbf{h}$ CRW curves of WT and EPOR-cKO mice exposed to normoxia and $\mathbf{i}$ quantification of their Ctip2 ${ }^{+}$cells in CA1 (normalized to NR controls). $\mathbf{j}$ CRW curves of WT and EPOR-cKO mice exposed to inspiratory hypoxia, and $\mathbf{k}$ quantification of their $\mathrm{Ctip}^{+}$cells in CA1 (normalized to NR controls). I Representative images of $\mathrm{cFos}^{+}$neurons (cyan) in NR and CRW mice exposed to normoxia or hypoxia (scale bar: 25 um). $\mathbf{m}$ Quantification of newly formed neurons (Ctip2+/tdTomato-) in NR and CRW mice exposed to normoxia or hypoxia. $\mathbf{n}$, o Quantification of active neurons classified as pre-existing neurons ( $\mathrm{CFos}^{+} / \mathrm{Ctip}^{+} / \mathrm{tdTomato}^{+}$) or as newly formed neurons ( $\mathrm{cFos}^{+} / \mathrm{Ctip}^{+} /$tdTomato $^{-}$) in CA1 of mice quantified in $\mathbf{m}$. p Presentation of the small percentage of pre-existing neurons among $\mathrm{cFos}{ }^{+}$neurons. Data given as mean $\pm S E M$; two-tailed Student's $t$-test (i \& $k$ ) or two-way ANOVA; mouse $n$ numbers in panels. Source data of graphs $\mathbf{b}, \mathbf{c}, \mathbf{e}, \mathbf{g}-\mathbf{k}$, and $\mathbf{m}-\mathbf{p}$ provided as Source Data File.

Hypoxia: The hypoxia chamber was designed in cooperation with Coy Laboratory Products Inc. (Grass Lake, MI, USA) with the dimensions $164 \mathrm{~cm} \times$ $121 \mathrm{~cm} \times 112 \mathrm{~cm}$. The system includes an air filtration system consisting of carbolime and activated charcoal. The oxygen and carbon dioxide levels were constantly detected and controlled via online monitoring. A gradual reduction of $3 \%$ oxygen per day resulted in $12 \%$ oxygen level in 3 days and was maintained until the end of the experiment. During the course of the experiment, mice were treated with EdU via drinking water as described above.

Complex running wheels (CRW). Post weaning, mice were single-housed and tamoxifen treatment was initiated for NexCreERT2::tdTomato mice (described above). NexCreERT2::tdTomato and NexCre::EpoR flifl mice were divided into four groups and monitored for 17 days. Groups included (1) normoxic room conditions (at $21 \% \mathrm{O}_{2}$ ) in standard cages, (2) normoxic conditions with voluntary running on (at $21 \%$ (3) hypoxic conditions (bypoxia chamber to $12 \% \mathrm{O}_{2}$ ) in standard cages, on CRW, (3) hypoxic conditions (hoxia cham (4) hypoxic conditions with voluntary running on CRW. CRW (TSE Systems, Bad Homburg, Germany) is characterized by randomized missing bars as previousl described ${ }^{26,41}$. The testing period of 17 days was followed by 1 week of normal conditions for all groups (no running, normoxia). The mice that were previously running (in normoxic or hypoxic conditions) were finally exposed again to CR
for $4 \mathrm{~h}$ (as cFos inducing challenge) before being sacrificed (Fig. $4 \mathrm{f}$ ). For ISH for $4 \mathrm{~h}$ (as cFos inducing challenge) before being sacrificed (Fig. 4f). For ISH
experiments, male WT mice (P55) were exposed to 5,9 , or $13 \mathrm{~h}$ of CRW (Fig. 3a). experiments, male WT mice (P55) were exposed to 5,9 , or $13 \mathrm{~h}$ of CRW (Fig. 3a).
For the experiment involving CAGCreERT2-ODD::tdTomato mice, animals were exposed to overnight complex wheel running (Fig. 3e). Running was voluntary at all times with ad libitum access to food and water. Control mice (no running) were housed in standard cages. Mice were sacrificed, perfused, and brains collected as described below. Running was tracked automatically via Phenomaster software (TSE Systems, Germany) for the whole day. Since mice are mainly night-active (TSE Systems, Germany) for the whole day. Since mice are mainly night-active
(dark phase), the total distance run between 6 p.m. and 6 a.m. was summarized for every individual animal. The total distance run on each night was normalized to the
average distance of each animal run in the first 3 nights (data expressed as $\%$ performance in relation to the first 3 nights). For the experiments involving EPO performance in relation to the first 3 nights). For the experiments involving EPO
treatment, mice were treated with EPO (5000IU $/ \mathrm{kg}$, 11 i.p. injections on alternate treatment, mice were treated with $\mathrm{EPO}(50001 \mathrm{U} / \mathrm{kg}, 11$ i.p. injections on alternate
days for 3 weeks), followed by 1 -week break. They were then exposed to $12 \mathrm{~h}$ of CRW overnight (Fig. $4 a-c)$. Data for this experiment are expressed as distance run summarized for every $30 \mathrm{~min}$.

Immunohistochemistry (IHC). Mice were anesthetized and perfused transcardially with $4 \%$ cold formaldehyde. Dissected brains were post fixed in $4 \%$ formaldehyde at $4{ }^{\circ} \mathrm{C}$ and equilibrated in $30 \%$ sucrose dissolved in phosphate-buffered saline (PBS) at $4^{\circ} \mathrm{C}$ overnight. Brains were then embedded in cryoprotectant (O.C.T. TM Tissue-Tek, Sakura) and stored at $-80^{\circ} \mathrm{C}$. Whole mouse brains were cut into $30 \mu \mathrm{m}$ thick coronal sections (coordinates from bregma: -1.34 to $-2.54 \mathrm{~mm}$ posterior) using a cryostat (Leica) and stored in a cryoprotective solution ( $25 \%$ ethylene glycol and $25 \%$ glycerol in PBS) at $-20{ }^{\circ} \mathrm{C}$ until further use. For analysis of dendritic spines and Map2 dendrites, the right hemisphere, destined to the neuronal structural analysis, was cut in $100 \mu \mathrm{m}$ coronal sections with a vibratome (Leica VT $1000 \mathrm{E}$, Leica), collected in three subseries and stored at $4^{\circ} \mathrm{C}$ in PB $0.1 \mathrm{M}$ with sodium azide $(0.05 \%)$.

For IHC, sections were permeabilized in PBS containing $0.3 \%$ Triton X-100 and blocked in $5 \%$ horse serum for $1 \mathrm{~h}$ at RT. Brain sections were incubated with primary antibodies in blocking solution overnight at $4^{\circ} \mathrm{C}$, followed by $2 \mathrm{~h}$ incubation of appropriate fluorophore-conjugated secondary antibodies in blocking solution containing 3\% horse serum (or 5\% normal donkey serum) and counterstained with 4',6-diamidino-2-phenylindole (DAPI). The sections were then mounted on SuperFrostPlus Slides (ThermoFisher) with Aqua-Poly/moun (Polysciences, Inc). Primary antibodies used were: anti-Ctip2 (1:500; Guinea pi polyclonal; SYSY 325005), anti-Map2 (1:1000; mouse monoclonal; Sigma M9942), anti-Tbrl (1:200; rabbit monoclonal; Abcam ab183032), anti-Tle4 (1:200; rabbit polyclonal; Abcam ab64833), anti-NeuN (1:500; mouse monoclonal; Millipore MAB377), and anti-cFos (1:1000; rabbit polyclonal; SYSY 226003). Secondary antibodies used were: Alexa488 anti-Guinea pig (1:500; Jackson Immuno Research 706-548-148) and Alexa635 anti-mouse (1:400; ThermoFisher A31575). Depending on the need for a triple or a quadruple staining, Alexa 405 anti-rabbit (1:1000; Abcam 175652) or Alexa647 anti-rabbit (1:500; ThermoFisher A31573) were used. Following IHC, sections were stained for EdU using Click-i'T TM EdU Alexa Fluor ${ }^{\mathrm{TM}} 647$ Imaging kit (ThermoFisher E10415), according to the manufacturer's instructions.

Golgi-Cox staining. Mice were anesthetized and decapitated. The brain was removed, shortly rinsed in PBS, and processed with the ND Rapid GolgiStain ${ }^{\mathrm{TM}}$ Kit (FDN After impregnation, the brains were embedded in 2\% Agar Agar in PBS and cut in $100 \mu \mathrm{m}$ coronal sections with a vibratome (Leica, VT 1000 S) and placed on gelatin-coated glass slides (FDNeuroTechnologies, PO101). The slices were stained and dehydrated as described in the kit protocol. Slices were mounted with Perimount (ThermoFisher) Imaging and analysis. Pyramidal neurons: Imaging was performed using the Andor Eclipse TiE microscope system (Nikon, Tokyo, Japan) with a $40 \times$ objective (Plan Apo $\lambda 40 \times, \mathrm{NA}=0.95$ ) to image the hippocampal layers. Ctip2 ${ }^{+}$cells among total neuron numbers were manually counted. Ctip $2^{+}$and tdTomato cells were characterized as newly generated neurons. Quantifications are expressed as number of newly generated neurons divided by the total area of CAl stratum pyramidale $\left(\mathrm{mm}^{2}\right)$. For Ctip2 staining, a total of 16 hippocampi with 8 sections per animal were used. Quadruple (tdTomato, Ctip2, cFos, and EdU) staining was acquired with a TCS SP5-II System (Leica) equipped with a $20 \times$ objective $(\mathrm{NA}=0.70)$. For total Ctip2 ${ }^{+}$counts, six hippocampi with three sections per animal were used. Quantifications for CRW mice were expressed as percentage of Ctip2 ${ }^{+}$cells normalized to their respective non-running controls. Whole hippocampi were imaged and analyzed by Fiji software. Neurons showing positive immunoreactivity for cFos were identified and quantified manually. These neurons were further subcFos were identifed and quantifed mankally. These neurons were further subcategorized into pre-existing $\left(\mathrm{CFos}^{+}, \mathrm{td} \mathrm{Omato}^{+}, \mathrm{Ctip}^{+}\right)$or newly generate

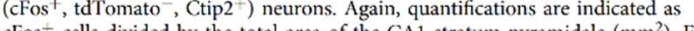
cFos ${ }^{+}$cells divided by the total area of the CA1 stratum pyramidale $\left(\mathrm{mm}^{2}\right)$. For cFos staining, a total of eight hippocampi with four sections per animal were used. For quantification of hypoxic neurons, whole hippocampus sections of $C A G$ CreERT2-ODD::tdTomato mice were imaged. The tdTomato ${ }^{+}$cells were characterized as neurons undergoing hypoxia and manually quantified. Quantifications are expressed as number of hypoxic neurons divided by the total area of CAl stratum pyramidale $\left(\mathrm{mm}^{2}\right)$. A total of ten hippocampi with five sections per animal were used. The cFos ${ }^{+}$cells were also analyzed as described above. For $\mathrm{Map} 2^{+}$dendritic density analysis, the stratum radiatum of the $\mathrm{CA} 1$ region was imaged using a laser scanning confocal microscope (Leica TCS SPE). Per animal, three dorsal hippocampal slices were selected starting at bregma $-1.58 \mathrm{~mm}$. three dorsal hippocampal slices were selected starting at bregma $-1.58 \mathrm{~mm}$.
Confocal $z$-stacks $(0.38 \mu \mathrm{m}$ intervals) of whole sections were taken using a $63 \times$ fields of the dimension $36.67 \mu \mathrm{m} \times 36.67 \mu \mathrm{m}$ were analyzed in each image (total 36 fields of the dimension $36.67 \mu \mathrm{m} \times 36.67 \mu \mathrm{m}$ were analyzed in each image (total 36
fields per animal). Images were processed using Fiji software and Map2 ${ }^{+}$principal fields per animal). Images were processed using Fiji software and $\mathrm{Map}^{+}$principal
apical dendrites were manually quantified. The quantifications are expressed as number of Map2 $2^{+}$dendrites divided by the area of each image $\left(1344.7 \mu \mathrm{m}^{2}\right)$.

Dendritic spines: Images were captured using a $63 \times$ oil immersion objective $(\mathrm{NA}=1.40)$ and a $3.5 \times$ additional digital zoom to investigate the first $200 \mu \mathrm{m}$ of the principal apical dendrite in segments of $50 \mu \mathrm{m}$. Confocal $z$-stacks $(0.38 \mu \mathrm{m}$ 


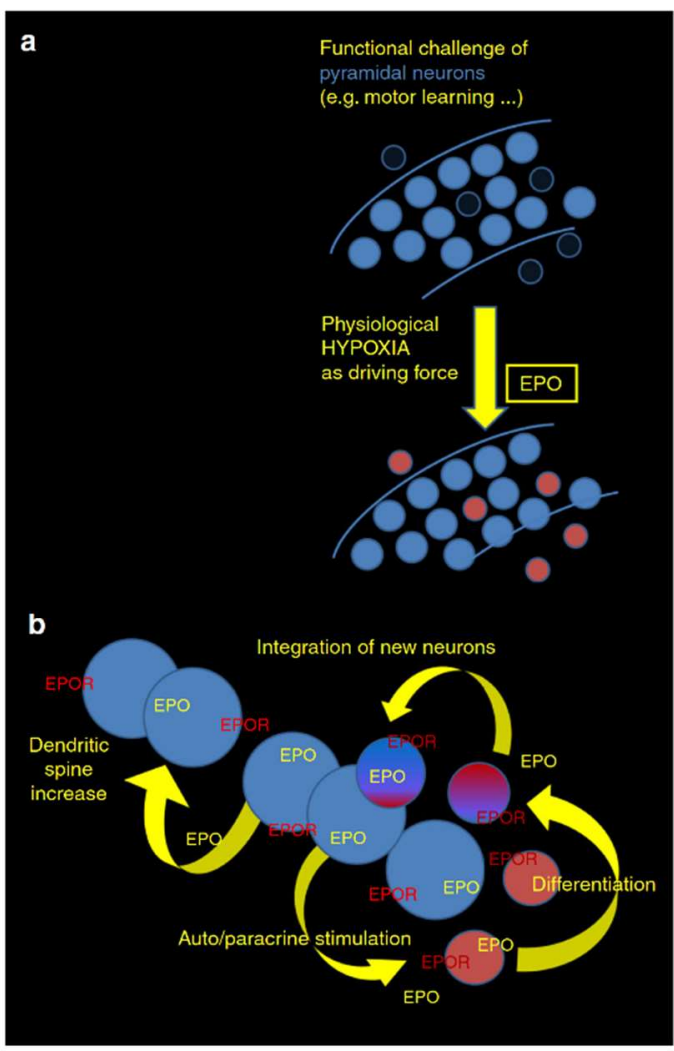

Fig. 5 Comprehensive working model of EPO function in the brain. Panels illustrate the suggested cellular mechanisms and effects of EPO/EPOR in CA1 (hypotheses and respective figure created by Hannelore Ehrenreich). a Functional challenge of pyramidal neurons (blue) via, e.g., learning of new complex tasks provokes physiological hypoxia of the involved neurons. Hypoxia in turn induces neuronal production/release of EPO, which initiates differentiation of diverse non-proliferating precursors (red) with currently unknown identity to neurons. b Neuronal EPO binds in an auto/paracrine manner to EPOR on pyramidal neurons (blue), leading to an increase in dendritic spines. EPO simultaneously binds to EPOR on likely diverse neighboring cells (red), ready to differentiate into neurons on demand. Together, these integrative steps may explain the consistently found improvement of cognitive function under rhEPO treatment and, even more importantly, delineate physiological mechanisms, accomplishing lasting adaptation to challenge via the brain EPO system.

intervals) of whole sections were performed. Dendrites included in the study wer at least $200 \mu \mathrm{m}$ in length. Per animal, six such dendrites of six different Thyl-YFP expressing pyramidal neurons were randomly selected from the $\mathrm{CA} 1$ region. The stitching plugin in Fiji software (2.0.0) was used to reconstruct three-dimensiona images of the apical dendrites. The spines were further sub-categorized as proxima $(0-50 \mu \mathrm{m})$, medial $(50-100 \mu \mathrm{m})$, medial-distal $(100-150 \mu \mathrm{m})$, and distal $(150-200 \mu \mathrm{m})$ segments of the dendrite, depending on their distance from the soma. The total density of spines was also analyzed. Based on their morphology ${ }^{23,52}$, the spines were manually divided into (1) stubby, i.e., length of he protusion was $<1$ m and no neck is observed (2) mushroom, when a he protrusion was $<1 \mu$ and head-like structure cold beast 1.5 times the average length of the neck) and the total length of the protrusion was
$<1.5 \mu \mathrm{m}$; and (3) thin, i.e., the length of the protrusion was $>1.5 \mu \mathrm{m}$ or the length was between 1 and $1.5 \mu \mathrm{m}$ and a clear head-like structure could not be distinguished.

For dendritic spine quantification using the Golgi-Cox method: Images were acquired using the Nikon Ti2 using a $100 \times$ objective $(\mathrm{NA}=1.45)$. Stretches of mi apical dendrites in CAl pyramidal neurons were recorded with a $z$-stack size of
$0.3 \mu \mathrm{m}$. Per animal, ten dendrites of ten different cells were quantified for the number of spines (calculated as spines per $\mu \mathrm{m}$ dendrite)

RNAscope in-situ hybridization (ISH): RNAscope ${ }^{*} 2.5$ HD Brown Reagent Ki (CatNo.322300), Advanced Cell Diagnostics (ACD), Hayward, CA, USA was used for the detection of EPO and EPOR mRNA. ISH was performed according to the manufacturer's instructions. Briefly, coronal cryosections of $15 \mu \mathrm{m}$ thickness were mounted on SuperFrostPlus Slides, dried, and stored at $-80^{\circ} \mathrm{C}$. Sections were the pretreated by dropwise addition of hydrogen peroxide and incubated for $10 \mathrm{~min}$ at RT Slides we immser in RI. Slides were ime incubation with proce plos for 30 min at with the corresponding target probe $\mathrm{Mm}-\mathrm{Epo}-01$ (CatNo.444941) or Mm-Epor (CatNo.41235I) for $2 \mathrm{~h}$ at $40^{\circ} \mathrm{C}$, followed by a series of amplification and washing steps. Chromogenic signal detection was performed with 3,3'-diaminobenzidin (DAB) incubation for $20 \mathrm{~min}$ at RT. Sections were counterstained with $50 \%$ Mayer's hemalum (Merck) and mounted with EcoMount (BioCare Medical). Brown punctate dots in the CAl were counted in a total of 12 hippocampi with 6 sections per animal using a light microscope (Olympus BX-50, Tokyo, Japan) equipped with a $100 \times$ oil immersion objective $(\mathrm{NA}=1.35)$ and normalized to the area of the respective region $\left(\mathrm{mm}^{2}\right)$. Sagittal $15 \mu \mathrm{m}$ sections from kidney (P55) and heart (E11.5) of WT mice were used as positive controls for EPO and EPOR, respectively. According to manufacturer's instructions, each dot represents a single molecule of mRNA in these sections. The quantification is normalized and presented as percentage of the mean dot number of groups and time points $\left[\%\right.$ value $=\left(\right.$ number $\left./ \mathrm{mm}^{2}\right) /\left(\right.$ mean dot number $\left.\left./ \mathrm{mm}^{2}\right) \times 100\right]$

Drop sequencing. Tissue dissociation: Juvenile male WT mice (P28; three mice/ group to allow biological replication) were injected i.p. with placebo or EPO and sacrificed after $6 \mathrm{~h}$. The hippocampi were dissected and sliced into $600 \mu \mathrm{m}$ sections using McIlwain Tissue chopper (Cavey Laboratory Engineering Co. Ltd). The CA1 region was digested with a working solution of Papain/DNaseI in Earle's Balanced Salt Solution, according to manufacturer's instructions (Worthington Biochemical Corp). The samples were then incubated at $37^{\circ} \mathrm{C}$ for 40 min with constant agitation before gentle manual trituration. The samples were centrifuged for $10 \mathrm{~min}$ at $200 \times g$ at $4^{\circ} \mathrm{C}$. After discarding the Papain/DNasel supernatant, cells were $200 \times g$ at $4^{\circ} \mathrm{C}$. After discarding the Papain/DNasel supernatant, cells were
resuspended in $1 \mathrm{~mL}$ of sterile DMEM/F12 (Sigma) without phenol-red containing $3 \%$ fetal bovine serum (FBS; Life Technologies) and the suspension was passed through a $40 \mu \mathrm{m}$ strainer cap (Corning) to yield a uniform single-cell suspension.

Single-cell barcoding and library preparation: Barcoded single cells, or STAMPs (single-cell transcriptomes attached to microparticles), and cDNA libraries were generated following the protocoll ${ }^{53}$. Briefly, single-cell suspensions (100 cells/ $\left./ \mathrm{l}\right)$, droplet generation oil (Bio-Rad), and barcoded microparticles (ChemGenes, 120 beads/ $/ \mathrm{l}$ ) were co-flowed through a FlowJEM aquapel-treated DropSec microfluidic device (FlowJEM) and droplets were generated for $15 \mathrm{~min}$. Captured RVA on the bead surface was recovered by washing the beads in saline-sodiur RNA on the bead salme-sodium citrate buffer and perflurooctanol solutions, and then reverse transcribed using Maxima $\mathrm{H}$ minus reverse transcriptase kit (ThermoFisher). Excess prime
surface of the bead uncaptured by an RNA molecule was digested using surface of the bead uncaptured by an RNA molecule was digested using
Exonucleases I kit (ThermoFisher). A cDNA amplification PCR was performed Exonucleases I kit ('ThermoFisher). A CDNA amplification PCR was performed
using $10 \mu \mathrm{M}$ SMART PCR primer and $2 \mathrm{X}$ Kapa HiFi HotStart ReadyMix (Kapa Biosystems) with 5000 beads per tube, and amplified for nine PCR cycles. The resulting samples were purified using AMPureXP beads (Beckman Coulter) and the quality and concentration of the cDNA was assessed using High-sensitivity DNA bioanalyzer (Agilent Technologies). Library sizes were adjusted using the Nexter Amplicon Tagmentase enzyme and DNA was amplified for 14 cycles using $10 \mu \mathrm{M}$ New-P5-SMAR'T PCR hybrid oligo, $10 \mu \mathrm{M}$ Nextera Index, and the Nextera PCR mix (Nextera XT DNA Library Preparation kit; Illumina). Tagmented libraries were again purified (AMPureXP), quality controlled (high-sensitivity DNA Bioanalyzer), quantified (Qubit dsDNA HS assay kit; Life Technologies), and Bioanalyzer, quantifed (Qubit dsDNA HS assay kit; tile Technologies), sequenced (Illumina $\mathrm{Hi}_{\mathrm{i}}$-seq 2500). All assays mentic

Single-cell RNA-seq processing: Unique molecular identifier (UMI) gene counts for each group (placebo or EPO) was imported into R (v3.4.1). Seurat (v2.3.0) function within the $\mathrm{R}$ environment was used for filtering, normalization, canonical correlation analyses (CCA), unsupervised clustering, visualization, and differential expression analyses.

Filtering and data normalization: Cells with minimum and maximum of 1000 and 8000 genes expressed ( $\geq 1$ count), respectively, and the genes that were expressed in at least three cells were retained. Cells with $>40 \%$ of counts on mitochondrial genes were excluded (Supplementary Fig. 1). After filtering, there were 14,061 genes in 390 cells from placebo and 14,971 genes in 583 cells from EPO group.

Gene UMI counts for each cell were normalized via natural-log normalization of gene UMI counts divided by total UMI counts per cell and scaled by 10,000. After normalization, scaled expression ( $z$-scores for each gene) for downstream After normalization, scalc

Canonical correlation analysis: Integration of scRNA-seq data from the two groups (placebo and EPO) was performed using CCA. Top 1000 highly variable genes from each group were used to calculate canonical correlation vectors (reduced dimension) and subsequently, first 20 vectors were aligned using dynamic time warping. 
Clustering, visualization, and differential expression: Clustering was performed using "FindClusters" function with default parameters with resolution set to 1 and first $20 \mathrm{CCA}$ aligned dimensions were used in the construction of the sharednearest neighbor graph and to generate two-dimensional embeddings for dat visualization using ISNE. Based on the visualization the glutamatergic cluster 0 and 1 were merged manually to represent a single cluster. The percentages of 'immature
glutamatergic cells' for each mouse were: placebo $1.6 \%, 2.3 \%$, and $4.3 \% ;$ EPO $3.7 \%$, glutamatergic cells for each mouse were: placebo $1.6 \%, 2.3 \%$, and $4.3 \%$; EPO $3.7 \%$, $6.8 \%$, and $7.0 \%$. We used the "Find Allmarkers" function with default parameters
and tested genes with a detected threshold of minimum of $25 \%$ of cells in either of and tested genes with a detected threshold of minimum of $25 \%$ of cells in
the two clusters. Genes with an adjusted $p<0.01$ were considered to be the two clusters. Genes with an adjusted $p<0.01$ were considered to be
differentially expressed (for a list of cluster markers see Source Data File). A differentially expressed (for a list of cluster markers see Source Data File). A
heatmap showing the top ten marker genes, i.e., differentially expressed genes per cluster is provided in the Supplementary Fig. 2.

Cell trajectory (pseudotime) analysis: Trajectory analysis of cells from the 'Immature Glutamatergic' and 'Mature Glutamatergicl' clusters $(n=502)$ was performed in Monocle ${ }^{25}$. The trajectory was constructed according to the documentation of Monocle2. Prior to cell ordering, reclustering was performed to confirm robust detection of the immature cluster across methods, which revealed three clusters (one cluster largely corresponding to Seurat's 'Immature Glutamatergic' cluster and two corresponding to Seurat's 'Mature Glutamatergicl' cluster; Supplementary Fig. 3a). Subsequently, dimension reduction using the 'DDRTree' method was performed. Differentially expressed genes $(q<0.01)$ DDRTree method was performed. Differentially expressed genes $(q<0.01)$
between the three clusters obtained by reclustering in Monocle 2 were used as input for pseudotemporal ordering.

Quantification and statistical analysis. All statistical analysis was performed using GraphPad Prism 5. For comparisons across multiple groups, a two-way analysis of variance (ANOVA) was used. For comparisons across two groups, an unpaired Student's $t$-test was performed. A $p<0.05$ is considered statistically sigunpaired Student's $t$-test was performed. A $p<0.05$ is considered statistically
nificant. Variance was similar between compared groups for their respective nificant. Variance was similar between compared groups for their respective
experiments. All values represent mean \pm SEM (standard error of the mean). All analysis and quantification were performed in a double-blinded fashion.

Reporting summary. Further information on research design is available in the Nature Research Reporting Summary linked to this article.

\section{Data availability}

The data that support this study are available from the corresponding authors upon reasonable request. A Source Data File is provided. Raw and processed scRNA-seq data are available via the GEO with accession code GSE144444.

\section{Code availability}

Analyis

Analysis scripts are accessible from

Received: 15 October 2019; Accepted: 12 February 2020; Published online: 09 March 2020

\section{References}

1. Krantz, S. B. Erythropoietin. Blood 77, 419-434 (1991)

2. Jelkmann, W. Regulation of erythropoietin production. J. Physiol. $\mathbf{5 8 9}$ $1251-1258$ (2011)

3. Digicaylioglu, M. et al. Localization of specific erythropoietin binding sites in defined areas of the mouse brain. Proc. Natl Acad. Sci. USA 92, 3717-3720 (1995).

4. Marti, H. H. et al. Erythropoietin gene expression in human, monkey and murine brain. Eur. J. Neurosci. 8, 666-676 (1996).

5. Shingo, T., Sorokan, S. T., Shimazaki, T. \& Weiss, S. Erythropoietin regulates the in vitro and in vivo production of neuronal progenitors by mammalian forebrain neural stem cells. J. Neurosci. 21, 9733-9743 (2001).

6. Brines, M. \& Cerami, A. Emerging biological roles for erythropoietin in the nervous system. Nat. Rev. Neurosci. 6, 484 (2005).

7. Sirén, A.-L., Faßhauer, T., Bartels, C. \& Ehrenreich, H. Therapeutic potential of erythropoietin and its structural or functional variants in the nervous system. Neurotherapeutics 6, 108-127 (2009).

8. Schuler, B. et al. Acute and chronic elevation of erythropoietin in the brain improves exercise performance in mice without inducing erythropoiesis. FASEB J. 26, 3884-3890 (2012)

9. Ehrenreich, H. et al. Exploring recombinant human erythropoietin in chronic progressive multiple sclerosis. Brain 130, 2577-2588 (2007).

10. Ehrenreich, $\mathrm{H}$. et al. Improvement of cognitive functions in chroni schizophrenic patients by recombinant human erythropoietin. Mol. Psychiatry 12, 206 (2007)
11. Wüstenberg, T. et al. EPO treatment preserves gray matter in discrete brain regions of chronic schizophrenic patients: indication of areas with most progressive neurodegeneration inherent to the disease process. Mol. Psychiatry 16, 1 (2011)

12. Miskowiak, K. W. et al. Recombinant human erythropoietin for treating treatment-resistant depression: a double-blind, randonized, placebocontrolled phase 2 trial. Neuropsychopharmacology 39, 1399 (2014).

13. Miskowiak, K. W. et al. Effects of erythropoietin on hippocampal volume and memory in mood disorders. Biol. Psychiatry 78, 270-277 (2015).

14. Adamcio, B. et al. Erythropoietin enhances hippocampal long-term potentiation and memory. BMC Biol. 6, 37 (2008).

15. El-Kordi, A., Radyushkin, K. \& Ehrenreich, H. Erythropoietin improves operant conditioning and stability of cognitive performance in mice. $B M C$ Biol. 7, 37 (2009)

16. Sargin, D. et al. Expression of constitutively active erythropoietin receptor in pyramidal neurons of cortex and hippocampus boosts higher cognitive functions in mice. BMC Biol, 9, 27 (2011).

17. Hassouna, I. et al. Revisiting adult neurogenesis and the role of erythropoietin for neuronal and oligodendroglial differentiation in the hippocampus. Mol. Psychiatry 21, 1752 (2016)

18. Sirén, A.-L. et al. Erythropoietin prevents neuronal apoptosis after cerebral ischemia and metabolic stress. Proc. Natl Acad. Sci. USA 98, 4044-4049 (2001).

19. Digicaylioglu, M. \& Lipton, S. A. Erythropoietin-mediated neuroprotection involves cross-talk between Jak2 and NF-kB signalling cascades. Nature 412 641 (2001).

20. Byts, N. et al. Essential role for Stat5 in the neurotrophic but not in the neuroprotective effect of erythropoietin. Cell Death Differ. 15, 783 (2008).

21. Agarwal, A. et al. In vivo imaging and noninvasive ablation of pyramidal neurons in adult NEX-CreERT2 mice. Cereb. Corlex 22, 1473-1486 (2011).

22. Feng, G. et al. Imaging neuronal subsets in transgenic mice expressing multiple spectral variants of GFP. Neuron 28, 41-51 (2000).

23. Kasai, H., Fukuda, M., Watanabe, S., Hayashi-Takagi, A. \& Noguchi, J. Structural dynamics of dendritic spines in memory and cognition. Trends Neurosci. 33, 121-129 (2010).

24. Wu, Y. E., Pan, L., Zuo, Y., Li, X. \& Hong, W. Detecting activated cell populations using single-cell RNA-seq. Neuron 96, 313-329. e316 (2017)

25. Qiu, X. et al. Reversed graph embedding resolves complex single-cell trajectories. Nat. Methods 14, 979 (2017).

26. Liebetanz, D. et al. A highly sensitive automated complex running wheel test to detect latent motor deficits in the mouse MPTP model of Parkinson's disease. Exp. Neurol. 205, 207-213 (2007).

27. Kietzmann, T., Mennerich, D. \& Dimova, E. Y. Hypoxia-inducible factors (HIFs) and phosphorylation: impact on stability, localization, and transactivity. Front. Cell Dev. Biol. 4, 11 (2016).

28. Kimura, W. et al. Hypoxia fate mapping identifies cycling cardiomyocytes in the adult heart. Nature 523, 226 (2015)

29. Morgan, J. I., Cohen, D. R., Hempstead, J. L. \& Curran, T. Mapping patterns of $\mathrm{c}$-fos expression in the central nervous system after seizure. Science 237, 192-197 (1987)

30. Dessypris, E., Graber, S. E., Krantz, S. B. \& Stone, W. J. Effects of recombinant erythropoietin on the concentration and cycling status of human marrow hematopoietic progenitor cells in vivo. Blood 72, 2060-2062 (1988).

31. Grover, A. et al. Erythropoietin guides multipotent hematopoietic progenitor cells toward an erythroid fate. J. Exp. Med. 211, 181-188 (2014).

32. Brown, G. \& Ceredig, R. Modelling the hematopoietic landscape. Front. Cell Dev. Biol. 7, 104 (2019).

33. Manalo, D. J. et al. Transcriptional regulation of vascular endothelial cell responses to hypoxia by HIF-1. Blood 105, 659-669 (2005)

34. Zhao, W., Kitidis, C., Fleming, M. D., Lodish, H. F. \& Ghaffari, S. Erythropoietin stimulates phosphorylation and activation of GATA-1 via the PI3-kinase/AKT signaling pathway. Blood 107, 907-915 (2006).

35. Zhang, K., Zhu, L. \& Fan, M. Oxygen, a key factor regulating cell behavior during neurogenesis and cerebral diseases. Front. Mol. Neurosci. 4, 5 (2011)

36. Dale, E., Ben Mabrouk, F. \& Mitchell, G. Unexpected benefits of intermittent hypoxia: enhanced respiratory and nonrespiratory motor function. Physiology 29, 39-48 (2014).

37. Cao, L. et al. VEGF links hippocampal activity with neurogenesis, learning and memory. Nat. Genet. 36, 827 (2004).

38. Miskowiak, K., O'Sullivan, U. \& Harmer, C. J. Erythropoietin enhances hippocampal response during memory retrieval in humans. J. Neurosci. 27, $2788-2792(2007)$

39. Cao, J. et al. The single-cell transcriptional landscape of mammalian organogenesis. Nature 566, $496(2019)$.

40. Kesner, R. P., Lee, I. \& Gilbert, P. A behavioral assessment of hippocampal function based on a subregional analysis. Rev. Neurosci. 15, 333-352 (2004).

41. McKenzie, I. A. et al. Motor skill learning requires active central myelination. Science 346, 318-322 (2014) 
42. Altman, J. \& Das, G. D. Autoradiographic and histological evidence of postnatal hippocampal neurogenesis in rats. J. Comp. Neurol. 124, 319-335 (1965).

43. Kaplan, M. S. \& Hinds, J. W. Neurogenesis in the adult rat: electron microscopic analysis of light radioautographs. Science 197, 1092-1094 (1977)

44. Rakic, P. Adult neurogenesis in mammals: an identity crisis. J. Neurosci. 22 614-618 (2002).

45. Doetsch, F. The glial identity of neural stem cells. Nat. Neurosci. 6, 1127 (2003).

46. Gould, E. How widespread is adult neurogenesis in mammals? Nat. Rev. Neurosci. 8, 481 (2007).

47. Sanai, N. et al. Corridors of migrating neurons in the human brain and their decline during infancy. Nature 478, 382 (2011)

48. Aimone, J. B. et al. Regulation and function of adult neurogenesis: from genes to cognition. Physiol. Rev. 94, 991-1026 (2014)

49. Hughes, E. G., Kang, S. H., Fukaya, M. \& Bergles, D. E. Oligodendrocyte progenitors balance growth with self-repulsion to achieve homeostasis in the adult brain. Nat. Neurosci. 16, 668 (2013).

50. Muzumdar, M. D., Tasic, B., Miyamichi, K., Li, L. \& Luo, L. A global doublefluorescent Cre reporter mouse. Genesis 45, 593-605 (2007).

51. Goebbels, S. et al. Genetic targeting of principal neurons in neocortex and hippocampus of NEX-Cre mice. Genesis 44, 611-621 (2006).

52. Guirado, R. et al. The dendritic spines of interneurons are dynamic structures influenced by PSA-NCAM expression. Cereb. Cortex 24, 3014-3024 (2013).

53. Macosko, E. Z. et al. Highly parallel genome-wide expression profiling of individual cells using nanoliter droplets. Cell 161, 1202-1214 (2015).

\section{Acknowledgements}

This study was supported by the Max Planck Society, the DFG Research Center for Nanoscale Microscopy and Molecular Physiology of the Brain (CNMPB), and the Spanish Ministry of Economy and Competitiveness to J.N. (SAF2015-68436-R). U.J.B. received a PhD stipend from National University of Sciences and Technology (NUST) Faculty Development Program Abroad 2014/15 Pakistan. A.A.S. has held a stipend of the IMPRS-GGNB Ph.D. Program Neurosciences (DFG grant GSC 226) at the GeorgAugust-University, Göttingen. K.-A.N. is supported by the Adelson Medical Research Foundation and an ERC Advanced Grant. The authors are thankful for the technical support provided by the staff of the in-house light microscopy facility.

\section{Author contributions}

Concept, study design, and supervision: H.E., together with K.-A.N. and J.N. Experimental design and Interpretation: H.E., together with D.W. and F.S. Data acquisition and analysis: D.W., F.S., Y.C., U.J.B., V.B., A.A.S.-K., L.W., A.R., S.A., A.S., I.H., K.S., A.Q.I. M.Z., H.W., H.M., K.M., S.M.W., S.B., K.-A.N., J.N., and H.E. Drafting manuscript: H.I together with D.W. and F.S. Drafting display items: H.E. together with D.W., F.S., and Y.C. All authors read and approved the final version of the manuscript.

\section{Competing interests}

H.E. has submitted/holds user patents for EPO in stroke, schizophrenia, and M.S. Henrik Martens is an employee of Synaptic Systems GmbH. Apart from that, the authors declare no competing interests.

\section{Additional information}

Supplementary information is available for this paper at https://doi.org/10.1038/s41467020-15041-1.

Correspondence and requests for materials should be addressed to K.-A.N. or H.E.

Peer review information Nature Communications thanks the anonymous reviewers fo their contribution to the peer revicw of this work. Peer reviewer reports are available.

Reprints and permission information is available at http:/www.nature.com/reprints

Publisher's note Springer Nature remains neutral with regard to jurisdictional claims in published maps and institutional affiliations.

(c) (i) Open Access This article is licensed under a Creative Commons Attribution 4.0 International License, which permits use, sharing, adaptation, distribution and reproduction in any medium or format, as long as you give appropriate credit to the original author(s) and the source, provide a link to the Creative Commons license, and indicate if changes were made. The images or other third party material in this article are included in the article's Creative Commons license, unless indicated otherwise in a credit line to the material. If material is not included in the article's Creative Commons license and your intended use is not permitted by statutory regulation or exceeds the permitted use, you will need to obtain permission directly from the copyright holder. To view a copy of this license, visit http://creativecommons.org/ licenses $/$ by $/ 4.0 \%$.

(c) The Author(s) 2020 


\section{SUPPLEMENTARY INFORMATION}

Functional hypoxia drives neuroplasticity and neurogenesis via brain erythropoietin

Wakhloo et al 
Wakhloo et al Supplementary Figure 1

a
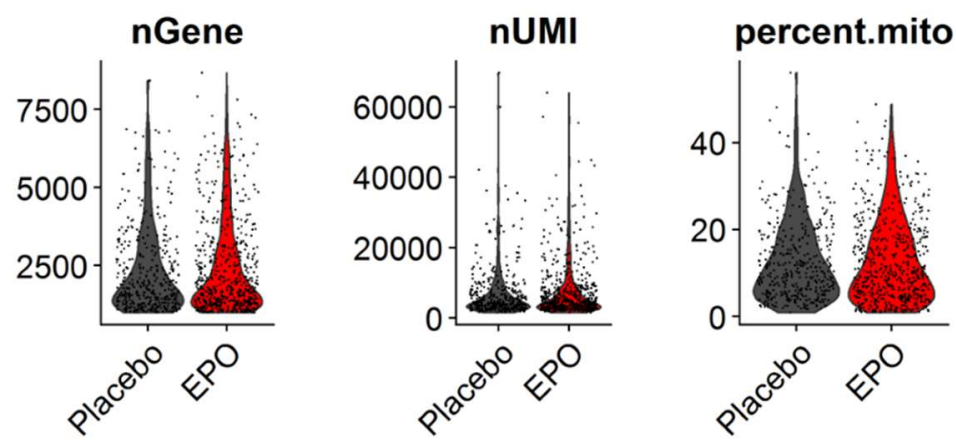

b

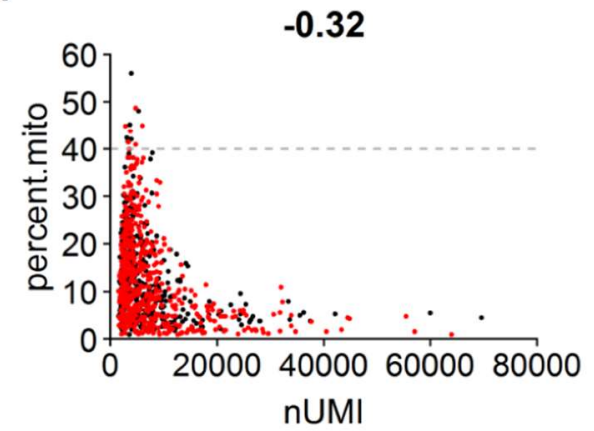

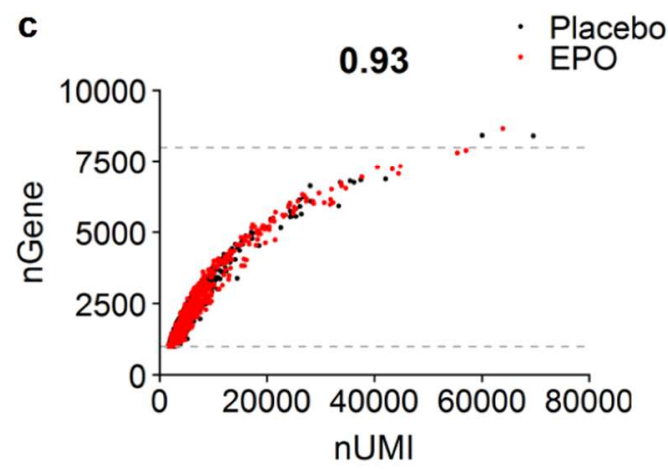

\section{Supplementary Figure 1: DropSeq quality control information}

Quality control information on pre-filtered (genes expressed in $\geq 3$ cells, cells expressing $\geq 1000$ genes) raw data from 6 male mice (P28). (a) Placebo and EPO treated groups are widely comparable with regard to major quality control readouts: number of genes detected (nGene), number of UMls detected (nUMI) and percentage of mitochondrial genes expressed. (b) Correlation of percentage of mitochondrial genes expressed (percent.mito) and number of UMls detected (nUMI), colored by treatment group. Cells above the dashed grey line were discarded before downstream analysis. (c) Correlation between number of genes ( $\mathrm{nGene}$ ) and number of UMls detected (nUMI), colored by treatment group. Cells outside the upper and lower dashed grey lines were discarded before downstream analysis. 
Wakhloo et al Supplementary Figure 2

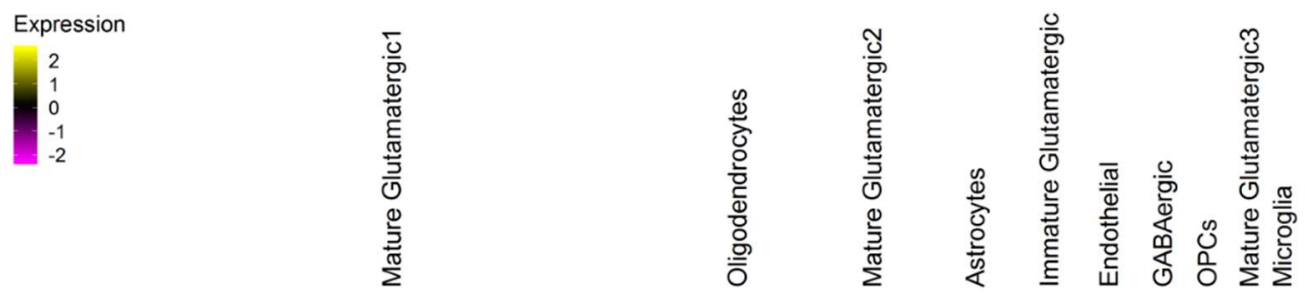

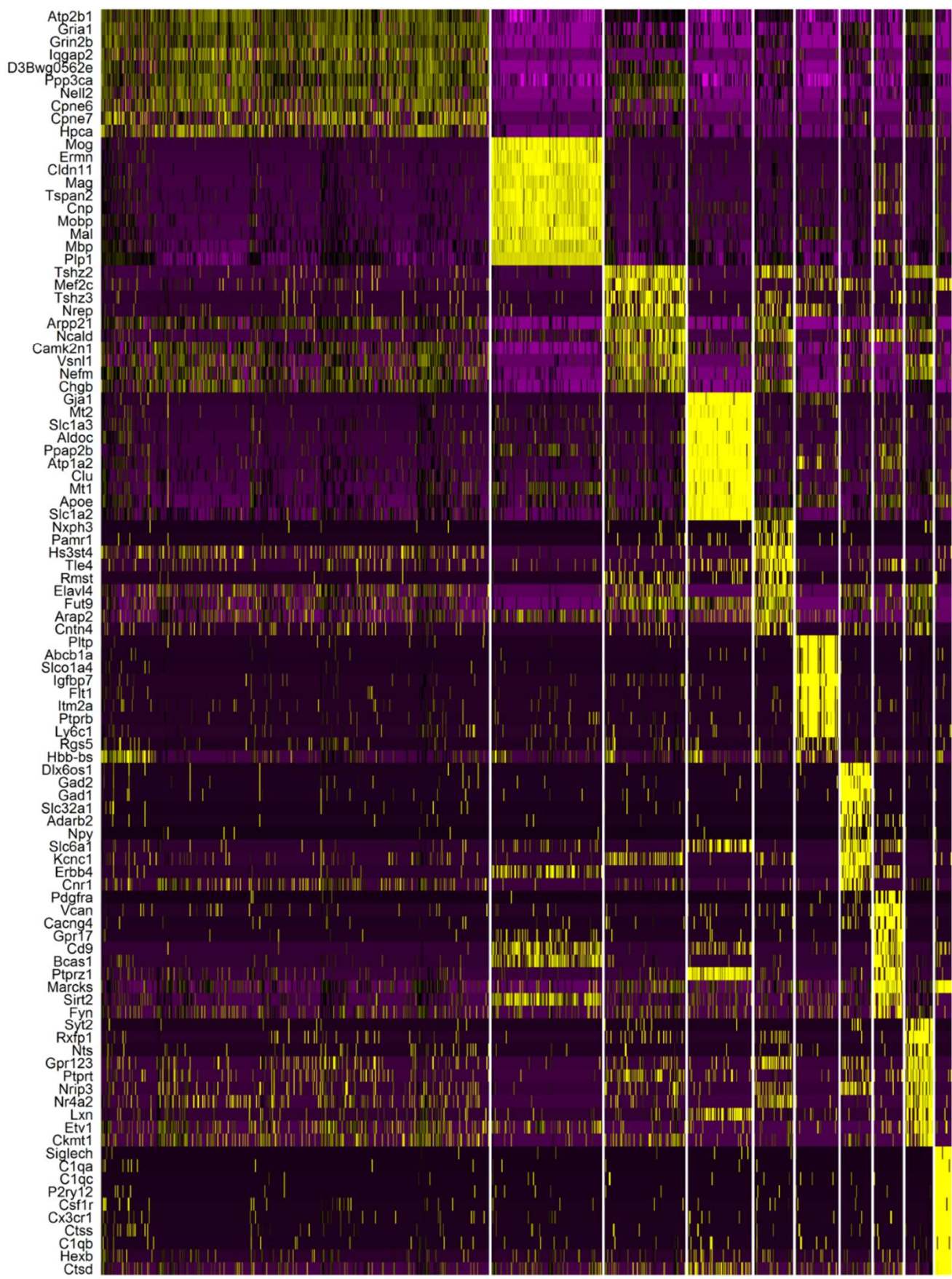

Supplementary Figure 2: Heatmap of top 10 marker genes (ordered by average log fold-change) per cluster. 
Wakhloo et al Supplementary Figure 3

a

Cluster - $1 \cdot 2 \cdot 3$

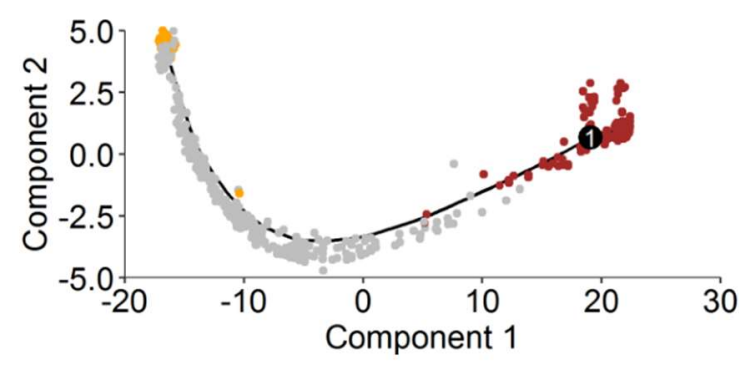

b

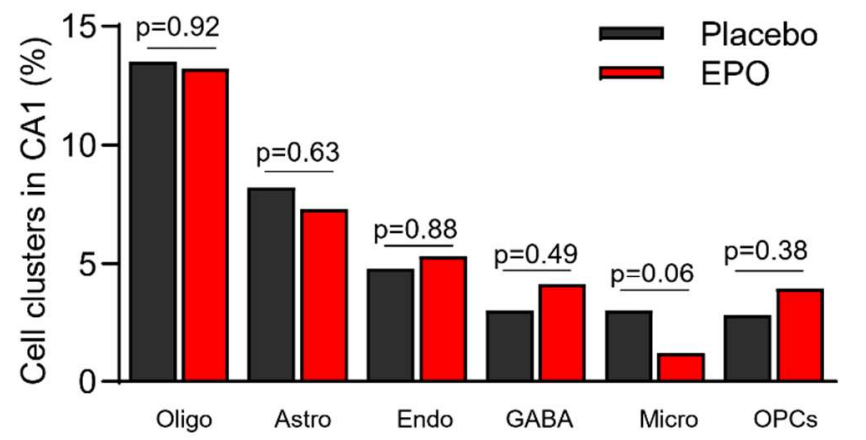

Legend

Oligo: Oligodendrocytes

Astro: Astrocytes

Endo: Endothelial cells

GABA: GABAergic neurons

Micro: Microglia

OPCs: Oligodendrocyte percursor cells

C

\begin{tabular}{llllllllll}
\hline & & \multicolumn{7}{c}{ \% positive cells } \\
\hline & Tbr1 & Dcx & Tle4 & Any & Triple+ & Tbr1+Dcx & Tbr1+Tle4 & Tle4+Dcx \\
\hline $\begin{array}{l}\text { Immature } \\
\text { (pseudotime >15) }\end{array}$ & 60 & 30 & 100 & 100 & 20 & 20 & 60 & 30 \\
$\begin{array}{l}\text { Immature } \\
\text { (pseudotime <15) }\end{array}$ & 33.3 & 33.3 & 69.4 & 88.9 & 8.3 & 13.9 & 25 & 16.7 \\
\hline \begin{tabular}{l} 
Mature \\
\hline
\end{tabular} & 9.6 & 8.6 & 18.6 & 30.7 & 0.7 & 1.8 & 3.3 & 1.8 \\
\hline
\end{tabular}

d

$\log 10($ value +0.1$)$

$-1.0-0.50 .00 .51 .0$

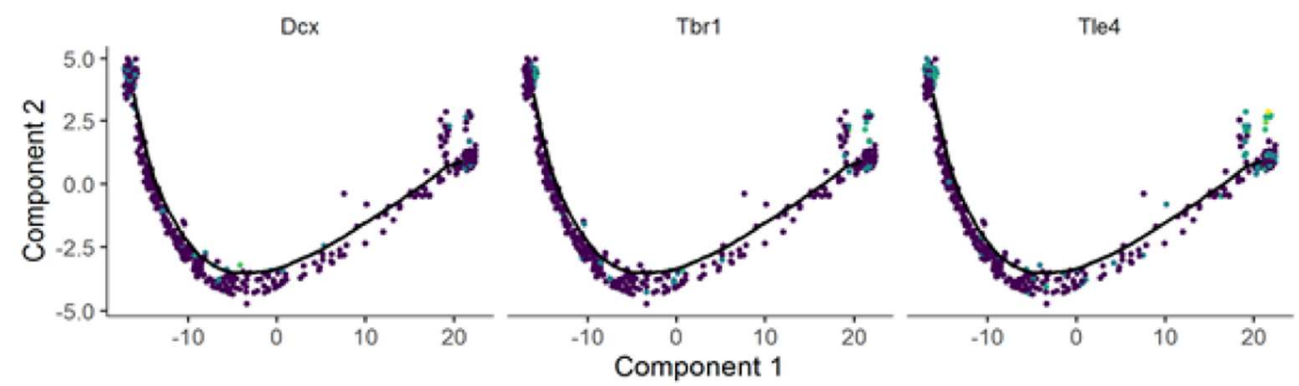




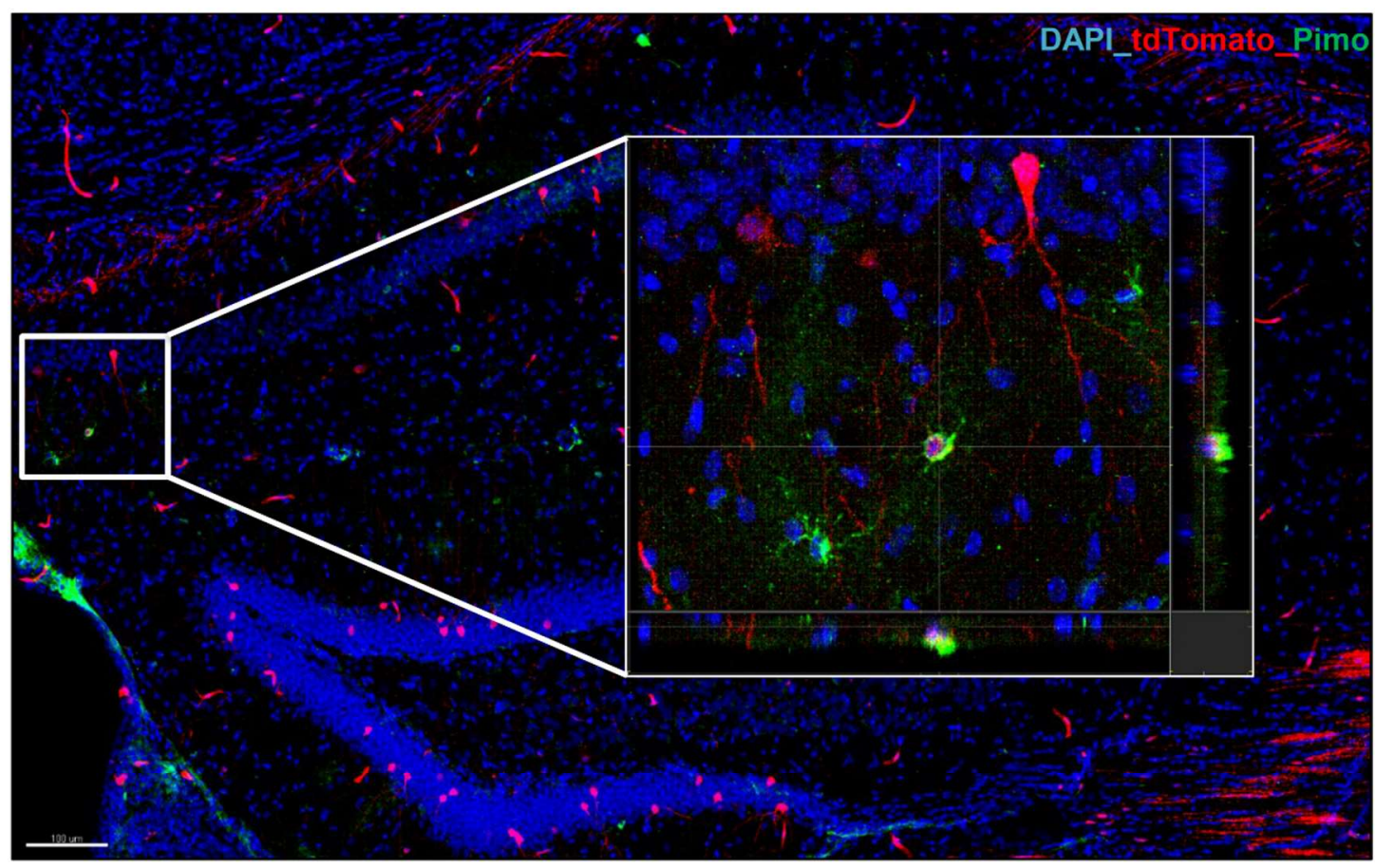

Supplementary Figure 4: Validation of hypoxia by pimonidazole staining

Hypoxic cells were validated with pimonidazole staining (according to Sato et al 2011) in CAG-CreERT2-ODD::R26R-tdTomato mice. Mice received a single tamoxifen injection (100 mg $/ \mathrm{kg}$ body weight), followed after 16 hours by pimonidazole administration, and were sacrificed $90 \mathrm{~min}$ later. Double-labeled hypoxic cells occur typically dispersed (compare Kimura et al 2015). Scale bar represents $100 \mu \mathrm{m}$ in the overview image.

Pimonidazole (Hypoxyprobe-1 Mab1) from NPI (Belmont, MA USA) is used to detect hypoxia in cells and tissue. Pimonidazole (60 mg/kg body weight) solution is injected intravenously (tail vein) and staining performed according to manufacturer's instructions. 
Wakhloo et al Supplementary Figure 5
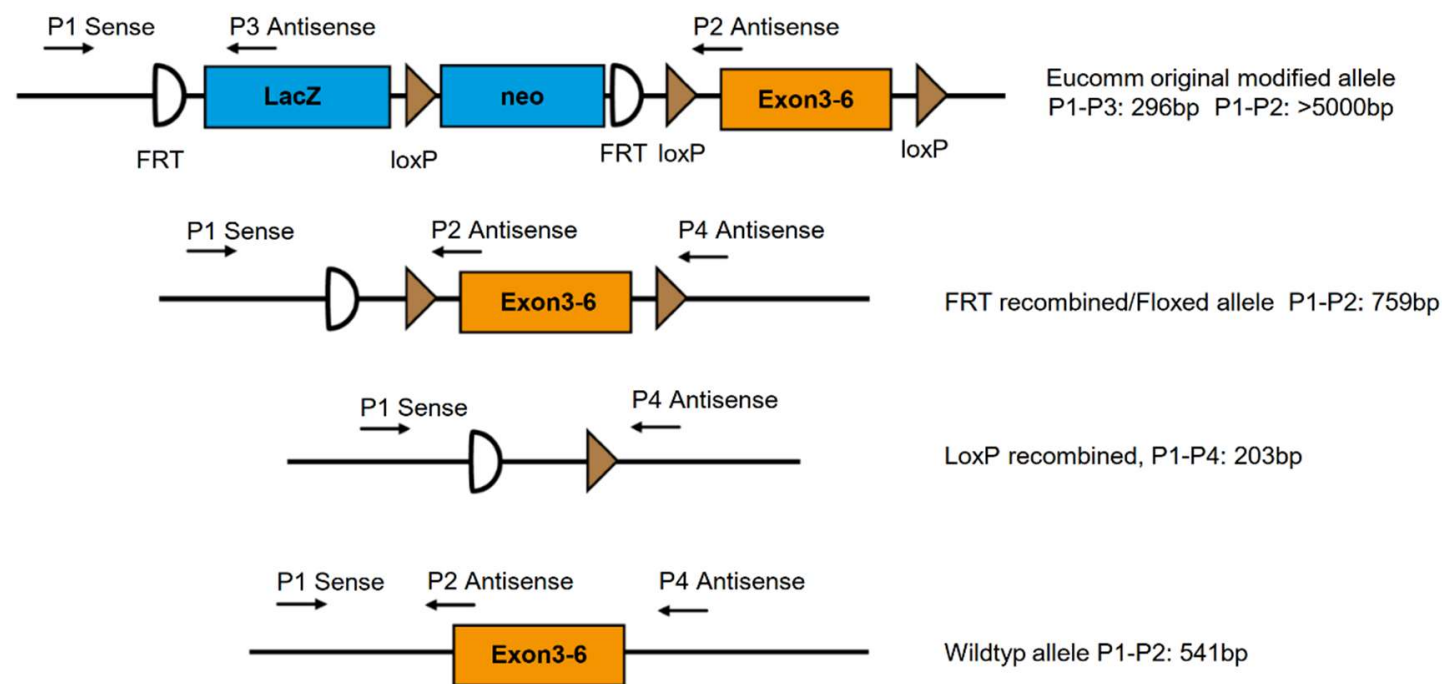

P1: 29825 5'-GTCCTCAGGACTGGGTGGC-3

P2: 29826 5'-CATCGATGTCGCTCCCAT-3'

P3: 15048 5'-CAACGGGTTCTTCTGTTAGTCC-3'

P4: 32002 5'-GAGCTCAGACCATAACTTCG-3'
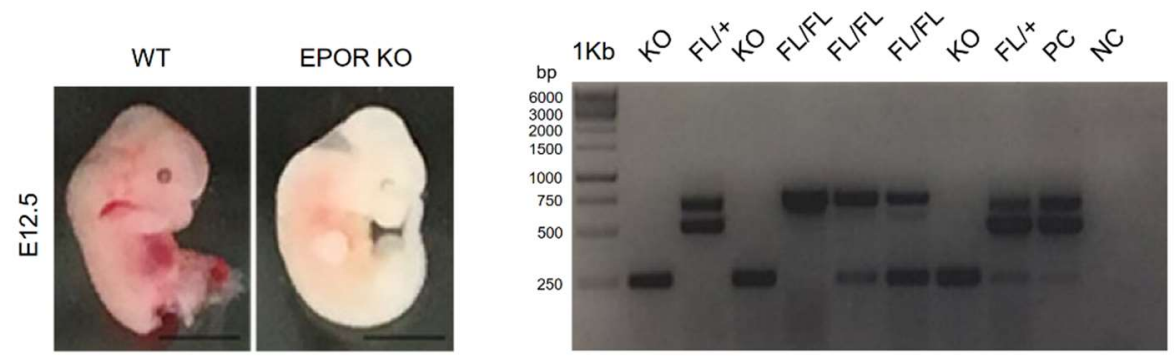

\section{Supplementary Figure 5: Description of pyramidal neuronal EPOR KO}

To generate the specific conditional EPOR $K O$ in pyramidal neurons (NexCre::EPORflox/flox), we had to first validate whether the generated EPOR KO mice are indeed functional knockouts. Therefore cross breeding of the female EPORflox/flox mice with male mice homozygous for the Cre-recombinase gene (under control of the ubiquitous adenovirus EllA-promoter), was used to obtain deleter mice. EllA-regulated Cre-recombinase was expressed in pre-implantation embryos leading to site specific deletion of LoxP flanked (fl) sequence in all tissues including germ cells. Interbreeding of first generation progenies resulted in efficient germline transmission of the deletion to subsequent generations. The presence of deleted alleles was determined by PCR-based genotyping using specific primers for EPOR (listed below) and also further confirmed using EllA-cre primers. We were able to observe a global EPOR KO at E12.5, validating functionality of generated EPOR KO mice. P1-4 (primer 1-4); E12.5 (embryonic day 12.5); PC (positive control); NC (negative control). 


\section{Supplementary References}

Sato, Y., Endo, H., Okuyama, H., Takeda, T., Iwahashi, H., Imagawa, A., Yamagata, K., Shimomura, I. \& Inoue, M. Cellular hypoxia of pancreatic $\beta$-cells due to high levels of oxygen consumption for insulin secretion in vitro. Journal of Biological Chemistry 286, 12524-12532 (2011).

Kimura, W., Xiao, F., Canseco, D. C., Muralidhar, S., Thet, S., Zhang, H. M., Abderrahman, Y., Chen, R., Garcia, J. A., Shelton, J. M., Richardson, J., Abderrahman, A., Aroumougame, A., Liang, H., Xing, C., Lu, Z., Zhang, C. \& Sadek, H. Hypoxia fate mapping identifies cycling cardiomyocytes in the adult heart. Nature 523, 226 (2015). 